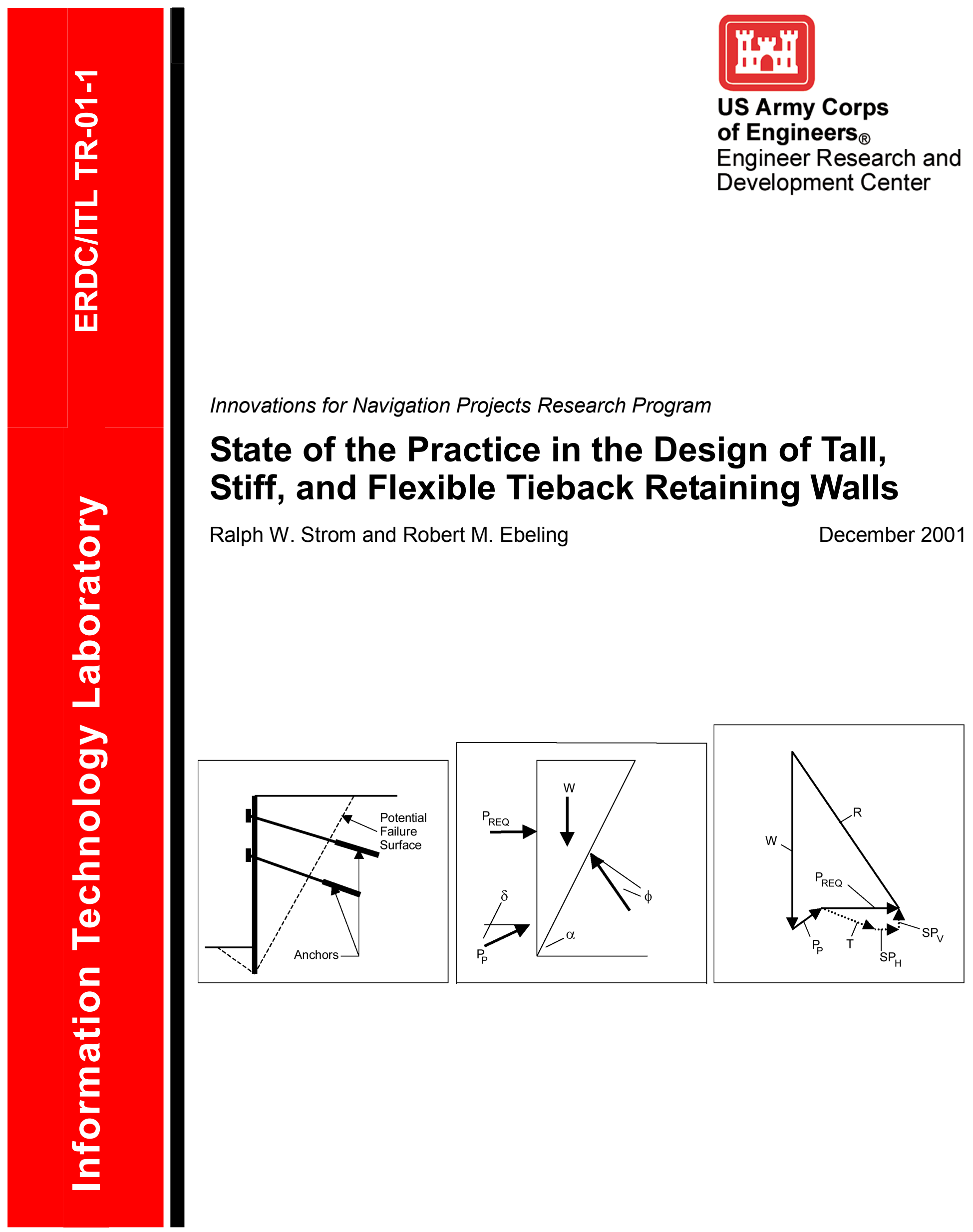


The contents of this report are not to be used for advertising, publication, or promotional purposes. Citation of trade names does not constitute an official endorsement or approval of the use of such commercial products.

The findings of this report are not to be construed as an official Department of the Army position, unless so designated by other authorized documents. 


\title{
State of the Practice in the Design of Tall, Stiff, and Flexible Tieback Retaining Walls
}

\author{
by Ralph W. Strom \\ 9474 SE Carnaby Way \\ Portland, OR 97266 \\ Robert M. Ebeling \\ Information Technology Laboratory \\ U.S. Army Engineer Research and Development Center \\ 3909 Halls Ferry Road \\ Vicksburg, MS 39180-6199
}

Final report

Approved for public release; distribution is unlimited

$\begin{array}{ll}\text { Prepared for } & \text { U.S. Army Corps of Engineers } \\ & \text { Washington, DC 20314-1000 } \\ \text { Under } & \text { INP Work Unit 33272 }\end{array}$




\section{Contents}

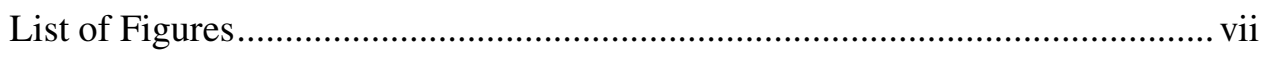

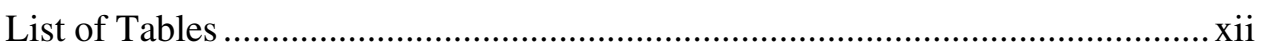

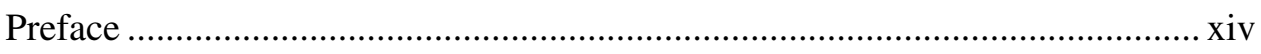

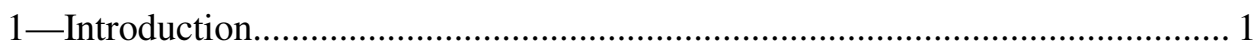

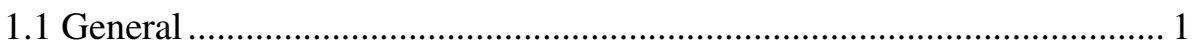

1.2 FHWA Research and Development on Anchored Walls ......................... 2

1.3 Application of FHWA Research to the Design of Corps Tieback

Wall Systems ......................................................................................... 3

1.4 Tieback Wall Deformations, Earth Pressures, and Drainage Effects ........ 3

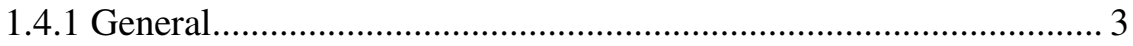

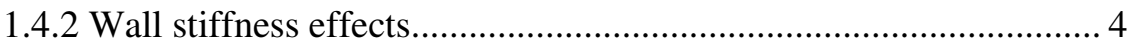

1.4.3 Relation between earth pressures and wall movements.................. 4

1.4.4 Construction short-term, construction long-term, and

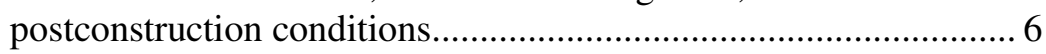

1.5 State of the Practice........................................................................ 9

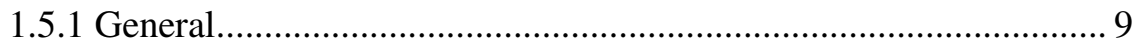

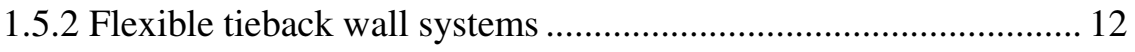

1.5.3 Stiff tieback wall systems ............................................................. 12

1.5.4 Common methods of tieback wall analysis ................................... 12

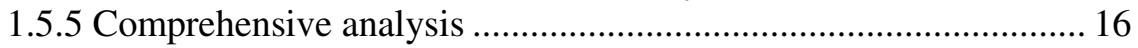

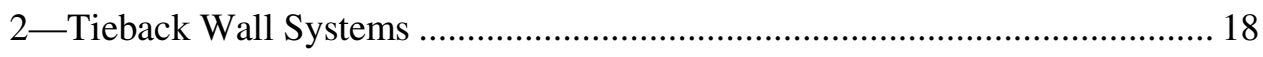

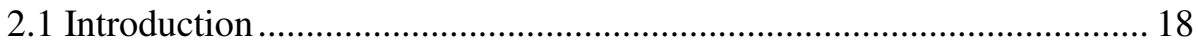

2.2 Vertical Sheet-Pile System with Wales and Post-Tensioned

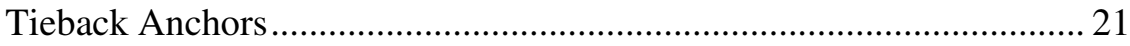

2.3 Soldier Beam System with Wood Lagging and Post-Tensioned

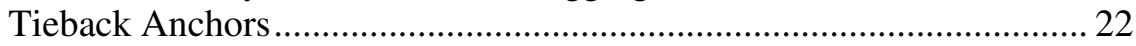

2.4 Secant Cylinder Pile System with Post-Tensioned Tieback Anchors ..... 23

2.5 Continuous Reinforced Concrete Slurry Wall System with Post-Tensioned Tieback Anchors ................................................... 24

2.6 Soldier Beam Tremie Concrete System with Post-Tensioned Tieback

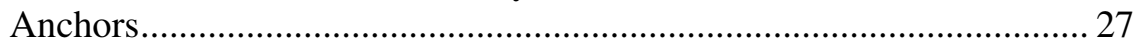

2.7 Continuous Versus Discrete Tieback Wall Systems .............................. 28

3-Tieback Wall Design and Design Standards............................................... 31

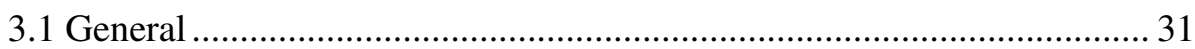

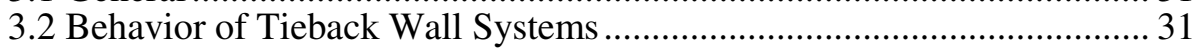

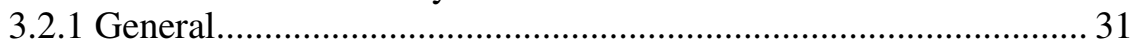


3.2.2 Tieback wall construction sequencing ........................................ 32

3.2.3 General behavior and design methodologies-focus

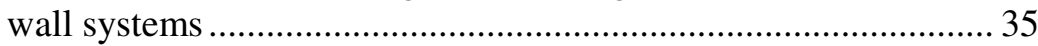

3.3 Tieback Wall Design and Analysis Procedures ..................................... 38

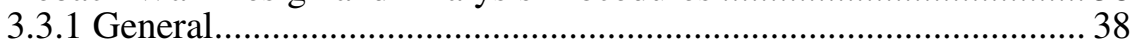

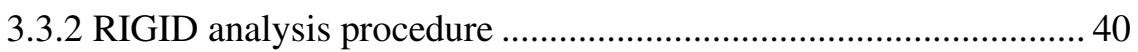

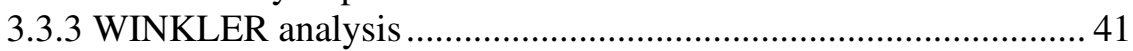

3.3.4 Linear elastic and nonlinear finite element method analyses ....... 42

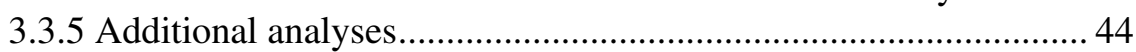

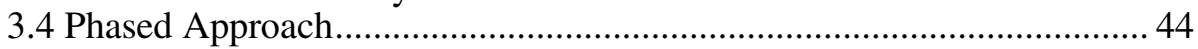

3.5 Performance Objectives ....................................................................... 46

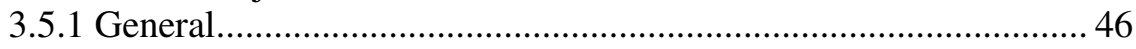

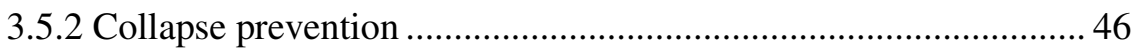

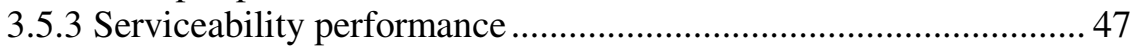

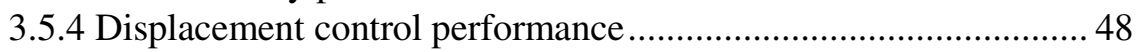

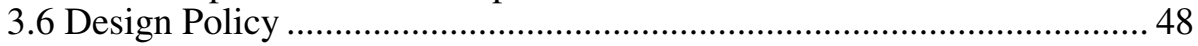

3.7 Combination of Loads ..................................................................... 48

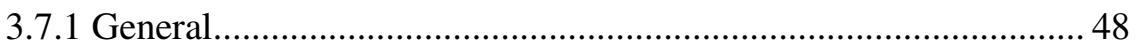

3.7.2 Allowable stress design of steel components .............................. 48

3.7.3 Ultimate strength design of reinforced concrete components........ 49

3.7.4 Special provisions for hydraulic structures..................................... 49

3.8 Applicable Design Codes and Standards............................................... 50

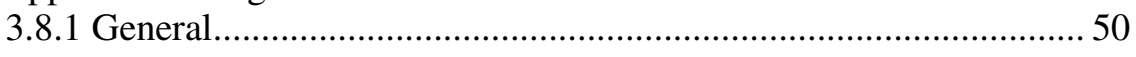

3.8.2 Special provisions of ASD applicable to tieback wall

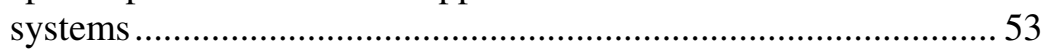

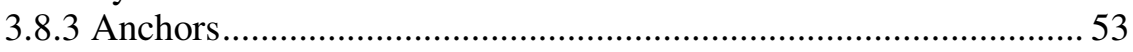

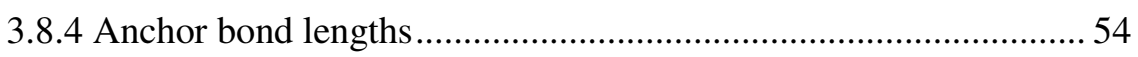

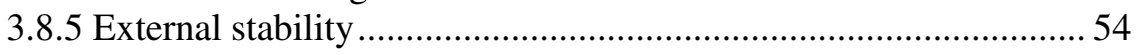

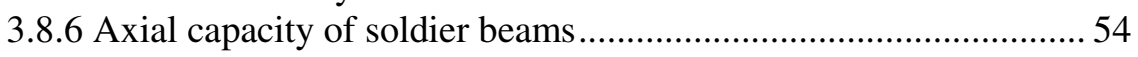

3.8.7 Tieback wall toe capacity ....................................................... 54

4-Geotechnical Investigations and Testing ….............................................. 55

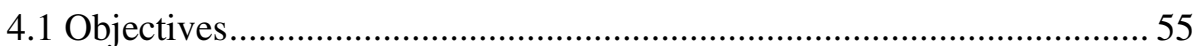

4.2 Subsurface Exploration and Site Characterization ................................. 56

4.3 Testing of Foundation Materials ………............................................... 56

4.4 In Situ Testing of Foundation Materials...................................................... 57

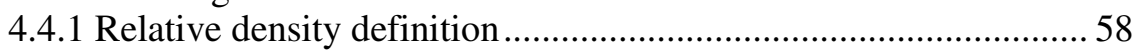

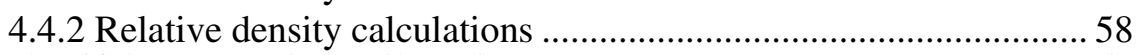

4.5 Identifying Corrosive Soil Environments............................................ 59

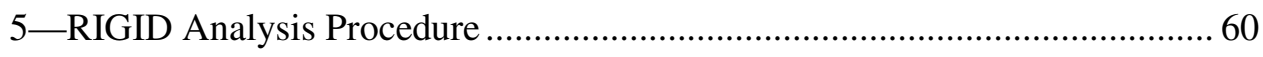

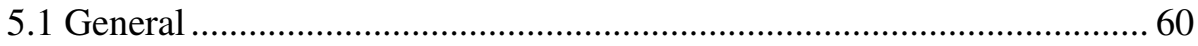

5.2 Apparent Versus Actual Earth Pressures................................................ 61

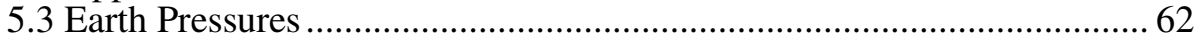

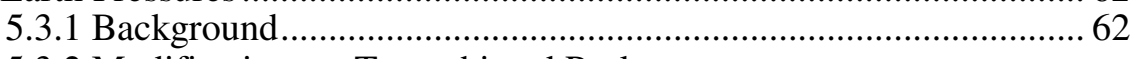

5.3.2 Modifications to Terazghi and Peck apparent pressure

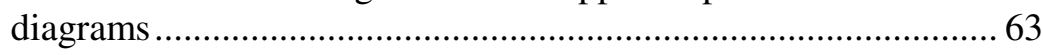

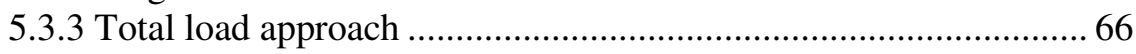

5.3.4 Apparent earth pressure diagrams for coarse-grained soils ........... 66

5.3.5 Apparent earth pressure diagrams for stiff clays ........................... 69

5.3.6 Apparent earth pressure diagrams for soft to medium clays.......... 70

5.3.7 Apparent earth pressure diagrams for soft to medium clays in soil subject to deep-seated failure ............................................... 72 
5.3.8 Total load by sliding wedge analysis........................................ 73

5.3.9 Total load approach for stiff wall systems................................... 75

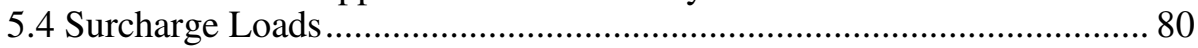

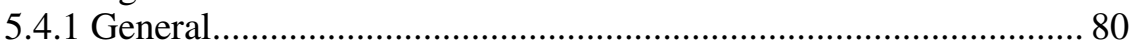

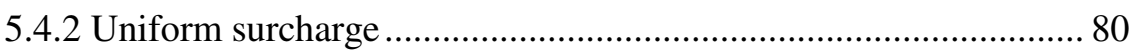

5.4.3 Point loads, line loads, and strip loads........................................ 81

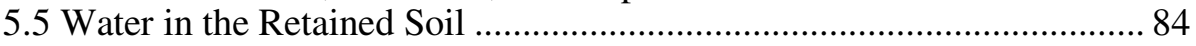

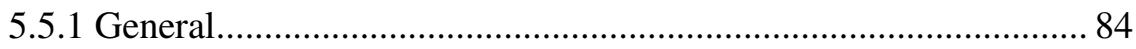

5.5.2 Combining earth and water pressures........................................ 84

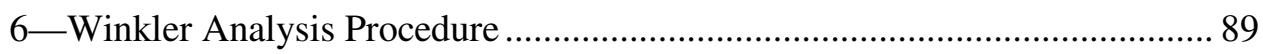

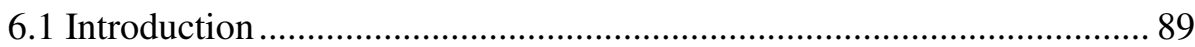

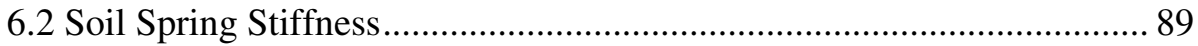

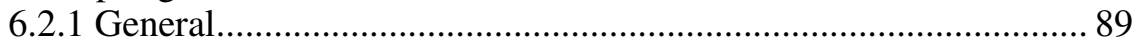

6.2.2 Coefficient of horizontal subgrade reaction................................. 92

6.2.3 Coefficient of horizontal subgrade reaction for discrete wall

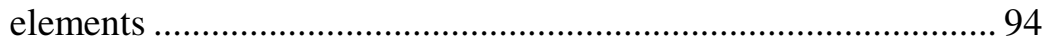

6.2.4 Subgrade reaction for continuous walls...................................... 95

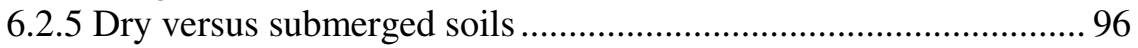

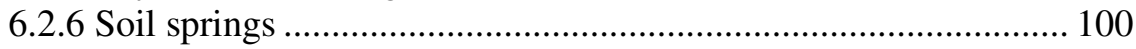

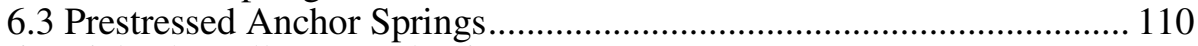

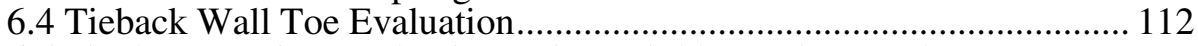

6.5 Final Excavation Evaluation Using Winkler Spring Analyses................ 113

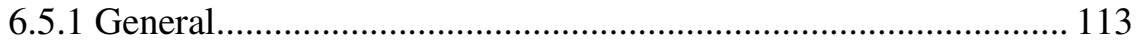

6.5.2 Tieback wall evaluation using Winkler spring analysis .............. 114

6.5.3 Winkler spring analysis results ................................................ 115

6.6 Simplified Construction Sequencing Evaluation-Shifted $R-y$

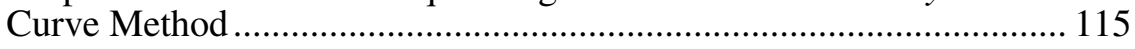

6.6.1 Approximate modeling of the excavation sequence ................... 115

6.6.2 Temporary tieback wall—Bonneville Navigation Lock.............. 120

6.7 Simplified Construction Sequencing Evaluation-Static Load Approach ................................................................................... 122

7-Finite Element Method (FEM) Analysis Procedure ................................... 124

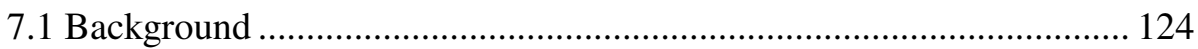

7.2 Common Types of Multi-Anchored Systems..................................... 125

7.3 Response of Soil-to-Wall Interfaces in Multi-Anchored Systems ........ 128

7.4 Gómez-Filz-Ebeling Interface Model ................................................... 131

7.5 Need for Nonlinear Finite Element SSI Analyses................................. 133

7.6 Bonneville Temporary Tieback Wall Analysis ..................................... 134

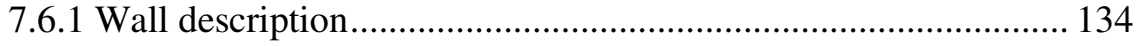

7.6.2 Overall design and evaluation process........................................ 134

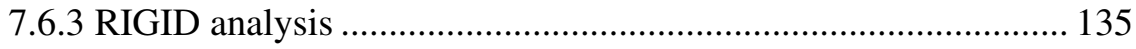

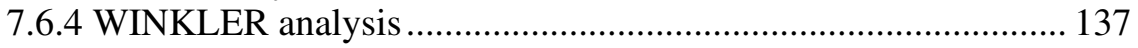

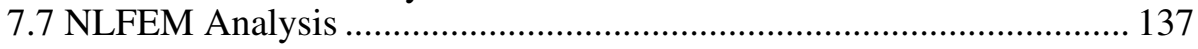

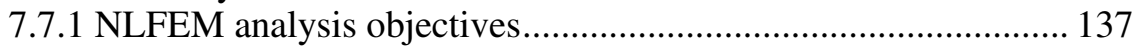

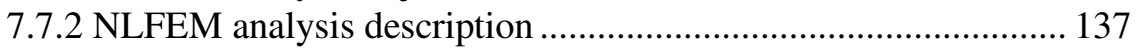

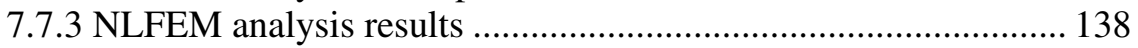

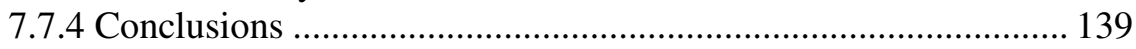

8-Design of Tieback Wall System Components and Wall Toe ..................... 143

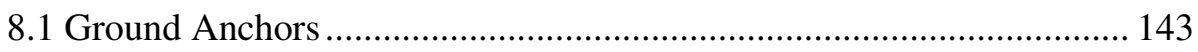

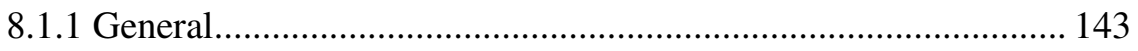




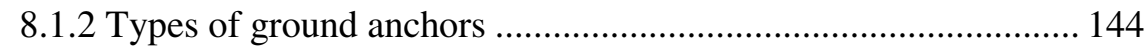

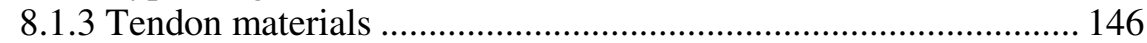

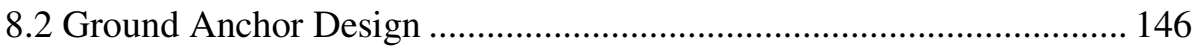

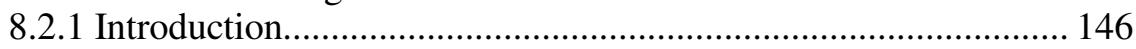

8.2.2 Location of critical potential failure surface ............................... 146

8.2.3 Calculation of ground anchor loads from apparent pressure

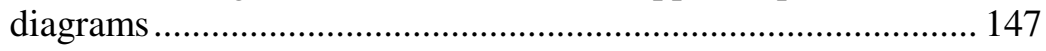

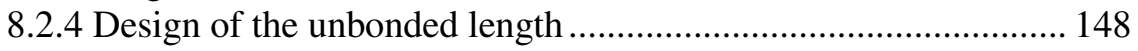

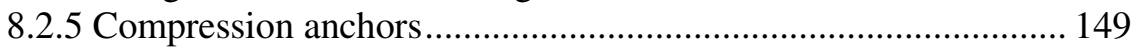

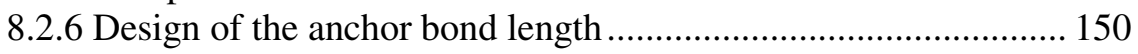

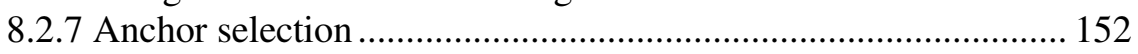

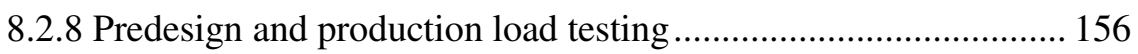

8.2.9 Spacing requirements for ground anchors ................................. 157

8.2.10 Selection of prestressing steel element .................................... 159

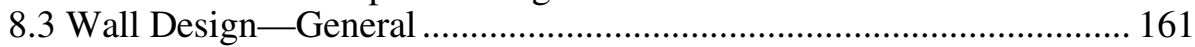

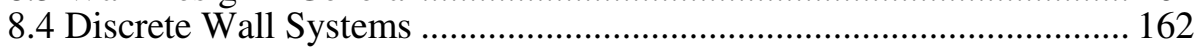

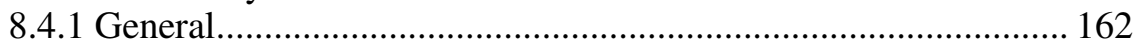

8.4.2 Allowable stress design for steel soldier beams .......................... 162

8.4.3 Wales and thru-beam connections ................................................ 163

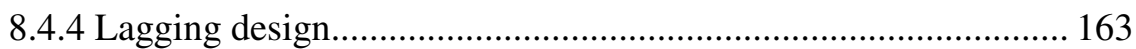

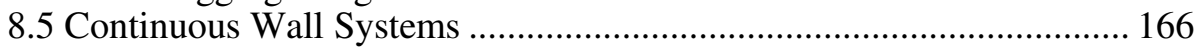

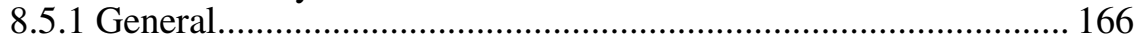

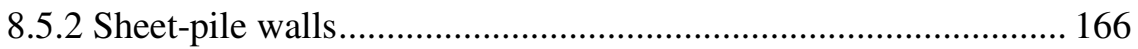

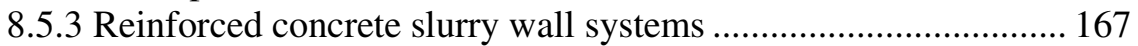

8.6 Permanent Facing Systems................................................................. 168

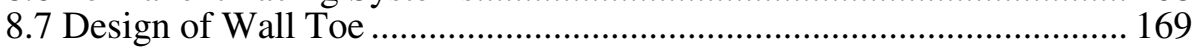

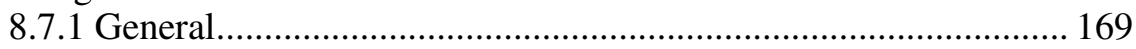

8.7.2 Lateral capacity for discrete wall systems ................................ 170

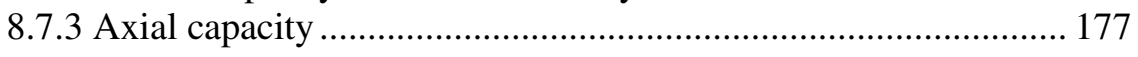

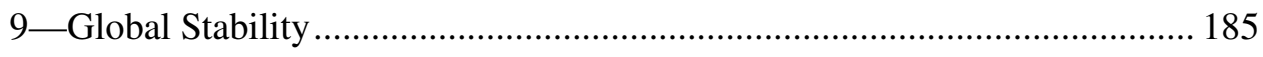

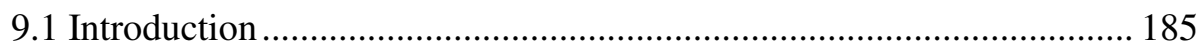

9.2 Stability of Anchored Wall Systems_Limit Equilibrium Methods ..... 185

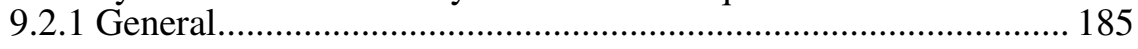

9.2.2 Evaluation of stability using limit equilibrium ........................... 187

9.2.3 Limit equilibrium calculations.................................................. 188

9.2.4 Modeling lateral wall resistance in limit equilibrium analysis .... 192

9.2.5 Comparison of methods to evaluate required earth loads in

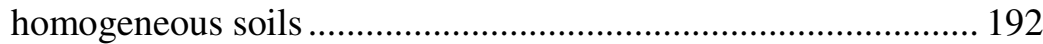

9.3 Base Stability and Heave in Cohesive Soils........................................ 195

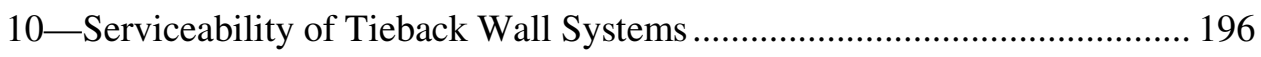

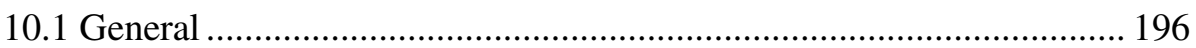

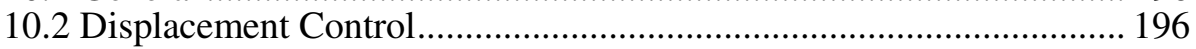

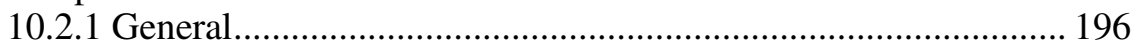

10.2.2 Estimating wall movements and ground settlements................ 196

10.3 Drainage and Seepage Control ........................................................ 197

10.4 Corrosion Considerations in Design .................................................... 198

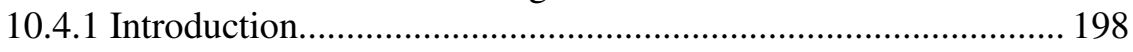

10.4.2 Corrosion and effects on ground anchors .................................. 199

10.4.3 Corrosion protection of ground anchors ................................... 200

10.4.4 Selection of corrosion protection level ..................................... 211

10.4.5 Corrosion of structural steel, cement grout, and concrete ......... 212 


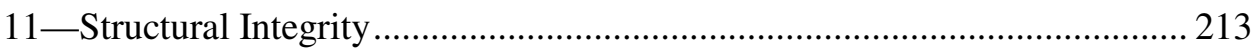

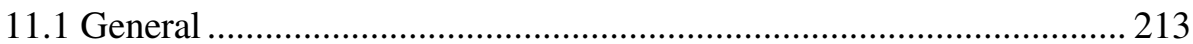

11.2 Testing of Anchor Systems ................................................................ 213

11.3 Redundant Systems and Detailing to Prevent Progressive Failures.... 215

11.4 Progressive Collapse and Plastic Collapse Mechanism Analyses....... 215

12 -Contracting for Tieback Wall Systems ...................................................... 216

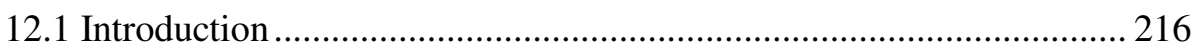

12.2 Method Contracting Approach......................................................... 217

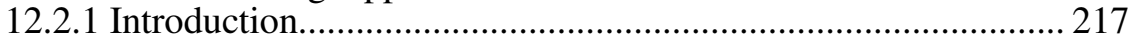

12.2.2 Contract documents for method approach .............................. 218

12.3 Performance Contracting Approach ................................................... 219

12.3.1 Introduction.............................................................................. 219

12.3.2 Implementing performance contracting approach ..................... 220

12.3.3 Contract documents for performance approach......................... 221

12.3.4 Review and approval ........................................................ 223

12.4 Contractor Design/Build Approach.................................................. 223

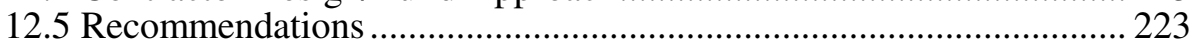

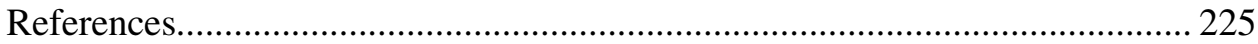

Appendix A: Relevant Design Standards, Specifications, and Computer Programs

SF 298

\section{List of Figures}

Figure 1.1. Relationship of earth pressure versus wall movements ...............5

Figure 1.2. Apparent earth pressures diagrams ......................................... 11

Figure 1.3. Equivalent beam on rigid supports method ............................ 13

Figure 1.4. Beam on elastic foundation method ......................................... 14

Figure 1.5. Linear elastic finite element model of diaphragm wall in combination with linear Winkler soil springs........................... 15

Figure 1.6. Nonlinear finite element method ............................................. 16

Figure 2.1. Vertical sheet-pile system with wales and post-tensioned tieback anchors ...................................................................... 19

Figure 2.2. Soldier beam system with timber lagging and posttensioned tieback anchors....

Figure 2.3. Secant cylinder pile system with post-tensioned tieback anchors 
Figure 2.4. Typical continuous concrete slurry wall system with post-tensioned tieback anchors.

Figure 2.5. Typical soldier beam tremie concrete wall system with post-tensioned tieback anchors

Figure 2.6. Vertical sheet-pile system with post-tensioned tieback anchors

Figure 2.7. Soldier beam system with wood lagging and posttensioned tieback anchors

Figure 2.8. Secant cylinder pile system with post-tensioned tieback anchors

Figure 2.9. Construction sequencing for concrete slurry wall system .........25

Figure 2.10. Continuous concrete slurry wall system with posttensioned tieback anchors

Figure 2.11. Soldier beam tremie concrete system with post-tensioned tieback anchors 28

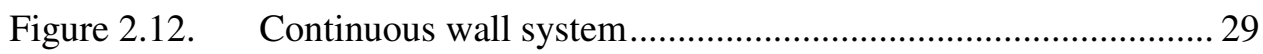

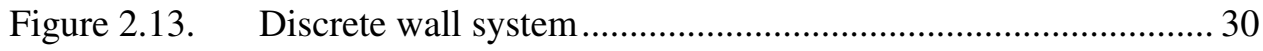

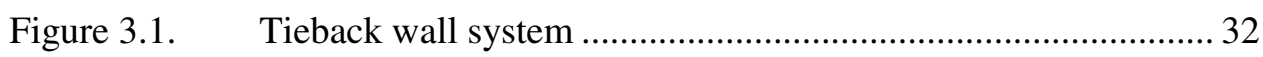

Figure 3.2. First-stage excavation-lateral wall movements and earth pressures ................................................................... 32

Figure 3.3. Upper tieback installation—lateral wall movements and earth pressures .................................................................. 33

Figure 3.4. Second-stage excavation-lateral wall movements and earth pressures ….................................................................... 33

Figure 3.5. Lower tieback installation final-stage excavationlateral wall movements and earth pressures ............................. 34

Figure 3.6. Trapezoidal earth pressure distribution ................................... 35

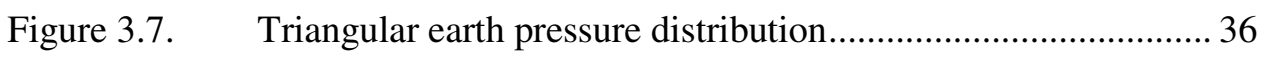

Figure 3.8. Composite earth pressure distribution .................................... 37

Figure 3.9. Linear elastic finite element model of diaphragm wall in combination with linear Winkler soil springs.......................... 42

Figure 3.10. Anchor prestress versus lateral wall movement and ground settlement 43

Figure 3.11. Anchor prestress versus soil pressure 43

Figure 3.12. Potential failure conditions to be considered in design of anchored walls..... 
Figure 5.1. Schematic model for redistribution of lateral stress due to wall movement

Figure 5.2. Apparent earth pressure diagrams .......................................... 62

Figure 5.3. Recommended apparent earth pressure diagram formulas for wall supported by one row of anchors

Figure 5.4. Recommended apparent earth pressure diagram formulas for wall supported by multiple rows of anchors

Figure 5.5. Earth pressure factors as a function of standard penetration resistance

Figure 5.6. Soft to medium clays-recommended apparent earth pressure diagram for wall supported by multiple rows of anchors.

Figure 5.7. Failure surface assumed for Henkel's method.......................... 72

Figure 5.8. Force equilibrium method for anchored walls........................... 74

Figure 5.9. Pressure distribution when toe movements are restricted.......... 77

Figure 5.10. Modeling for staged excavation analysis by RIGID method 78

Figure 5.11. Modeling for staged excavation analysis by nonrigid support method

Figure 5.12. Apparent earth pressure diagram—with uniform

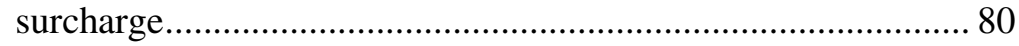

Figure 5.13. Increase in pressure due to point load.................................... 81

Figure 5.14. Increase in pressure due to line load.......................................... 82

Figure 5.15. Increase in pressure due to strip load..................................... 83

Figure 5.16. Method 2, effective unit weight for partially submerged soils.

Figure 5.17. Apparent earth pressure diagram with hydrostatic water

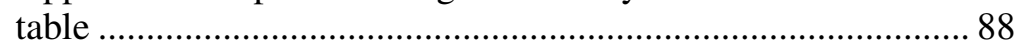

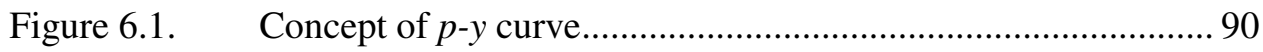

Figure 6.2. Tieback wall soil springs for Winkler analysis......................... 91

Figure 6.3. Subgrade reaction idealizations ................................................ 93

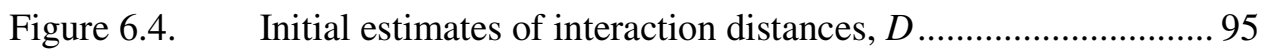

Figure 6.5. Subgrade reaction for a continuous wall, partially submerged conditions, hydrostatic water table in a granular soil 97 
Figure 6.6. Idealized elastoplastic earth response-deflection curve.

Figure 6.7. Diagram illustrating $R-y$ curves for tieback walls in cohesionless soils.

Figure 6.8. Diagram illustrating $R-y$ curves for tieback walls in cohesive soils

Figure 6.9. Diagram illustrating $P-y$ and $R-y$ single pile curves in clay

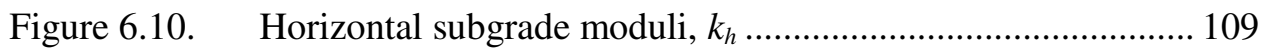

Figure 6.11. Ground anchor $T-y$ curve ...................................................... 111

Figure 6.12. Winkler spring model for toe analysis.................................. 112

Figure 6.13. Bonneville Navigation Lock temporary tieback wall .............. 113

Figure 6.14. Typical construction stages in a soil-structure interaction

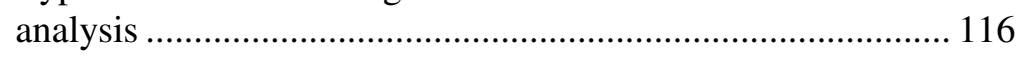

Figure 6.15. Diagram illustrating the $R-y$ curve for the cantilever

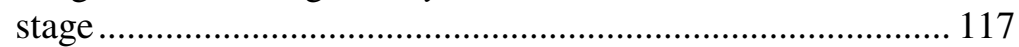

Figure 6.16. Diagram illustrating the $R-y$ curves at anchor stressing .......... 118

Figure 6.17. Shifted $R-y$ curve to model construction stages....................... 119

Figure 6.18. Diagram illustrating the $R-y$ curves during excavation below the ground anchor ..................................................... 120

Figure 6.19. Winkler staged analysis - shifted $R$ - $y$ curve method................ 122

Figure 6.20. Simplified construction sequencing evaluation—static load approach.................................................................. 123

Figure 7.1. Typical multi-anchored tieback wall system for Corps navigation project .............................................................. 126

Figure 7.2. Typical construction sequence of a reinforced concrete slurry wall ......................................................................... 127

Figure 7.3. Typical construction and operation stages............................. 129

Figure 7.4. Loading on soil-to-wall interface......................................... 131

Figure 7.5. Bonneville Navigation Lock, temporary tieback wall— horizontal section................................................................... 134

Figure 7.6. Bonneville Navigation Lock, temporary tieback wall—

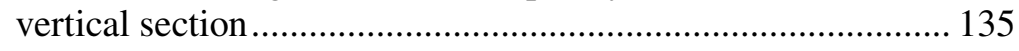

Figure 7.7. Overall design and evaluation process .................................. 136 
Figure 7.8. Deflections, moments, and earth pressures for Bonneville Navigation Lock, temporary tieback wallPanel 6 140

Figure 8.1. Components of a ground anchor........................................... 144

Figure 8.2. Main types of grouted ground anchors .................................. 145

Figure 8.3. Types of compression anchors............................................. 150

Figure 8.4. Mobilization of bond stress for a tension anchor in anchor bond zone............................................................... 153

Figure 8.5. Vertical and horizontal spacing requirements for ground

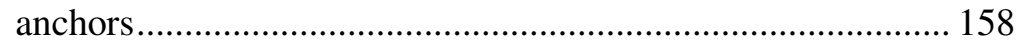

Figure 8.6. Passive wedge failure for a soldier beam in sand .................. 171

Figure 8.7. Intersecting failure wedges for soldier beams in sand............ 173

Figure 8.8. Plastic flow around a soldier beam toe ................................. 174

Figure 8.9. Passive wedge failure for a soldier beam in clay................... 175

Figure 8.10. Failure wedges for soldier beams in clay .............................. 176

Figure 8.11. Bearing capacity factor ...................................................... 181

Figure 8.12. Adhesion factor versus undrained shear strength ................... 182

Figure 9.1. Potential failure conditions to be considered in design of anchored walls ....................................................................... 186

Figure 9.2. Failure surfaces for external stability evaluations ................. 187

Figure 9.3. Modeling the ground anchor force in limit equilibrium analysis

Figure 9.4. Limit equilibrium analyses used to evaluate total lateral earth load for anchored systems constructed in weak cohesive soils

Figure 9.5. Comparison of limit equilibrium methods for cohesive soils

Figure 10.1. Settlement profile behind anchored walls............................. 197

Figure 10.2. Examples of corrosion protection for anchorages .................. 202

Figure 10.3. Examples of corrosion protection for classes I and II strand tendons

Figure 10.4. Examples of corrosion protection for classes I and II bar tendons 206 


\section{List of Tables}

Table 1.1. Approximate Magnitudes of Movements Required to Reach Minimum Active and Maximum Passive Earth Pressure Conditions

Table 3.1 Design Codes and Standards-Vertical Sheet-Pile System with Wales and P-T Tieback Anchors

Table 3.2 Design Codes and Standards-Soldier Beam System with Wood Lagging, P-T Tieback Anchors, and Permanent Concrete Facing

Table 3.3 Design Codes and Standards-Secant Cylinder Pile System with P-T Tieback Anchors 52

Table 3.4 Design Codes and Standards-Continuous Concrete Slurry Wall System with P-T Tieback Anchors

Table 3.5 Design Codes and Standards-Soldier Bream Tremie Concrete System with P-T Tieback Anchors............................. 53

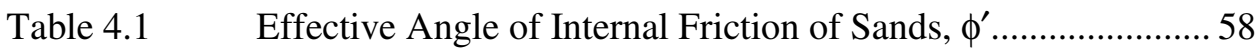

Table 5.1 Earth Pressure Factors for Typical Coarse-Grained Soils

Table 6.1 Estimated Values of the Constant of Horizontal Subgrade Reaction, Discrete Wall Systems in Moist and Submerged Sands

Table 6.2 Estimated Values of the Subgrade Constant for Continuous Wall Systems in Moist and Submerged Sands. 96

Table 6.3 Soil Parameters for Pfister Method Illustration ....................... 108

Table 7.1 Panel 6 Anchor Loads............................................................ 136

Table 7.2 Loading Steps in SOILSTRUCT Analysis ............................. 138

Table 8.1 Presumptive Ultimate Values of Load Transfer for Preliminary Design of Small-Diameter Straight Shaft Gravity-Grouted Ground Anchors in Soils. 152

Table 8.2 Presumptive Average Ultimate Bond Stress for Ground/Grout Along Anchor Bond Zone....

Table 8.3 Presumptive Ultimate Values of Load Transfer for Preliminary Design of Ground Anchors in Rock. 156

Table 8.4 Properties of Prestressing Steel Bars ...................................... 159

Table 8.5 Properties of Prestressing Steel Strands ................................. 160 
Table 8.6 Guidance on the Relationship Between Tendon Size and Trumpet Opening Size ............................................................. 160

Table 8.7 Recommended Thickness of Temporary Lagging.................... 165

Table 8.8 Maximum Design Bending Moments in Permanent

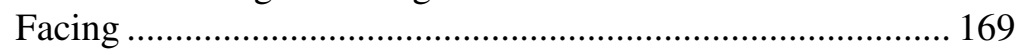

Table 8.9 Recommended Factors of Safety for Axial Capacity of Driven and Drilled-In Soldier Beams ....................................... 178

Table 8.10 Recommended Values of Unit Tip Bearing Capacity for Drilled Shafts in Sand .............................................................. 183

Table 9.1 Procedure to Evaluate Total Earth Load Using Slope Stability Computer Programs ................................................... 189

Table 9.2 Procedure to Evaluate Total Lateral Earth Load for Anchored Systems Constructed in Weak Cohesive Soils........ 191

Table 9.3 Values of $K_{R E Q}$ in Cohesionless Soils Using Various Methods to Evaluate Earth Pressures ....................................... 193

Table 10.1 Corrosion Protection Requirements........................................ 209 


\section{Preface}

The work described in this report was authorized by Headquarters, U.S. Army Corps of Engineers (HQUSACE), as part of the Innovations for Navigation Projects (INP) Research Program. The work was performed under Work Unit 33272, "Soil-Structure Interaction Studies of Walls with Multiple Rows of Anchors."

Dr. Tony C. Liu was the INP Coordinator at the Directorate of Research and Development, HQUSACE; Research Area Manager was Mr. Barry Holliday, HQUSACE; and Program Monitor was Mr. Mike Kidby, HQUSACE.

Mr. William H. McAnally of the U.S. Army Engineer Research and Development Center (ERDC) Coastal and Hydraulics Laboratory was the Lead Technical Director for Navigation Systems; Dr. Stanley C. Woodson, ERDC Geotechnical and Structures Laboratory (GSL), was the INP Program Manager.

This report was prepared by Mr. Ralph W. Strom, Portland, OR, and Dr. Robert M. Ebeling, ERDC Information Technology Laboratory (ITL). The work was monitored by Dr. Ebeling, Principal Investigator for INP Work Unit 33272, under the supervision of Mr. H. Wayne Jones, Chief, ComputerAided Engineering Division, ITL; Mr. Tim Ables, Acting Director, ITL; and Dr. Michael J. O'Connor, Director, GSL.

At the time of publication of this report, Dr. James R. Houston was Director of ERDC, and COL John W. Morris III, EN, was Commander and Executive Director.

The contents of this report are not to be used for advertising, publication, or promotional purposes. Citation of trade names does not constitute an official endorsement or approval of the use of such commercial products. 


\section{Introduction}

\subsection{General}

Tieback walls are often used for temporary support of excavations in cases where it becomes necessary to limit the area of the excavation in order to protect highways, railroads, structures, and other important man-made features that are located immediately adjacent to the excavation. In some instances the tieback wall system will remain as a permanent structure. Permanent tieback wall systems are used as guide walls and approach walls on navigation projects, and as retaining walls on highway and railroad protection and relocation projects. The structural design of tieback wall systems involves

- Selection of appropriate design and performance standards.

- Determination of the loadings and loading combinations to be used for design.

- Design of wall structural features such as tiebacks, soldier beams, wales, lagging, and facing systems.

- Detailing of all structural features to meet safety requirements and constructibility requirements.

- Selection of appropriate contract specifications to ensure that the completed structure meets all strength and serviceability requirements and provides corrosion protection suitable for the intended service life of the wall.

The state of the practice with respect to the design and evaluation of tieback wall systems is covered by this report. It is important to recognize that the subject matter covered herein is presented only as background information as to the state of the practice and as such is not in any way intended to represent Corps design guidance. No endorsement of the procedures described herein is implied or intended. The state of the practice will be illustrated with respect to the design and evaluation of five tieback wall systems. The systems selected (listed on following page) are described in detail in Chapter 2. 
- Vertical sheet-pile system with wales and post-tensioned tieback anchors.

- Soldier beam system with wood or reinforced concrete lagging and posttensioned tieback anchors. For the wood-lagging system, a permanent concrete facing system is required.

- Secant cylinder pile system with post-tensioned tieback anchors.

- Continuous reinforced concrete slurry wall system with post-tensioned tieback anchors.

- Discrete concrete slurry wall system (soldier beams with concrete lagging) with post-tensioned tieback anchors.

\subsection{FHWA Research and Development on Anchored Walls}

The use of tieback wall systems anchored with prestressed tendon-type ground anchors did not become commonplace until the late 1970s. The main use has been for the temporary and permanent support of highway cuts. Most research in the field of anchored tieback wall systems has therefore been under the auspices of the U.S. Department of Transportation Federal Highway Administration (FHWA). In 1979 the FHWA authorized a permanent ground anchor demonstration project. The objectives of this project were to provide highway agencies with adequate information to promote routine use of permanent ground anchors and anchored walls. The purposes of the demonstration project were to

- Study existing ground anchor technology and installation procedures.

- Determine additional areas where work was required.

- Update existing technology.

- Develop a basic design manual.

- Solicit installations on highway projects.

Many excellent research reports and design manuals were produced as a consequence of the FHWA research. A listing of these reports, and other relevant design standards, specifications, and computer programs, is provided in Appendix A.

This report draws heavily on information in the FHWA reports, in particular, Reports FHWA-SA-99-015 (Sabatini, Pass, and Bachus 1999) and FHWA-RD97-130 (Weatherby 1998a). Much of the information with respect to the design of flexible tieback wall systems has been taken verbatim from these FHWA documents and other FHWA research reports. 


\subsection{Application of FHWA Research to the Design of Corps Tieback Wall Systems}

The FHWA research and the information provided in FHWA research reports and design manuals is directly applicable to the design of flexible tieback wall systems such as (a) vertical sheet-pile systems with wales and posttensioned tieback anchors and (b) soldier beam system with wood or reinforced concrete lagging and post-tensioned tieback anchors. The other three tieback wall systems included in this report tend to be less flexible. Most flexible tieback wall systems displace sufficiently to mobilize active or near-active states of stress in the retained soil. In other words, the anchored wall moves away from the retained earth sufficiently to fully mobilize the shear resistance within the "soil wedge," or sufficiently to mobilize the resistance corresponding to a factor of safety of 1.0 on the shear strength of the soil.

Corps tieback wall systems, however, must often be designed to stringent displacement standards to protect critical railway transportation systems and important navigation, hydroelectric, and flood control structures and their operating systems from the damaging effects of ground settlements and lateral displacements. In addition, the tieback walls used on Corps projects often are higher than those commonly used for the support of highway cuts, and they are commonly subjected to operating conditions that make it difficult to keep the groundwater table below the base of the wall. With respect to the latter, the tieback walls used on Corps flood control and navigation projects are often required to resist boundary water-pressure loadings due to the presence of groundwater in addition to lateral earth pressures. This state-of-the practice report addresses the various issues related to the design of stiffer tieback wall systems and to the methods used to combine earth and water pressures, areas not well covered by the FHWA research. Still, much of the research performed by the FHWA has wide application with respect to the types of tieback wall systems typically found on Corps projects.

\subsection{Tieback Wall Deformations, Earth Pressures, and Drainage Effects}

\subsubsection{General}

Prestressing loads applied to tieback wall systems through the ground anchors restrict the movement of the wall system. The prestress load can be at a minimum level as required to prevent failure under active limit state conditions, or can be at significantly greater levels to limit wall displacements to levels consistent with project performance objectives. The deflected shape of the wall will depend on anchor locations, wall stiffness, and toe restraint conditions. The wall displacements that occur during construction may have a greater influence on design than those that occur after construction is complete. With this in mind, it is easy to understand why simple procedures have come to the forefront as the most commonly used approach for the design of tieback wall systems. 
These simple design procedures, commonly referred to as equivalent beam on rigid support procedures, are somewhat different with respect to the design of flexible and stiff wall systems. In general, they make assumptions with respect to wall-soil interaction, groundwater conditions, and the stresses that develop in the soil during and after construction. The designer must be familiar with all the assumptions made to ensure they are appropriate, keeping in mind that (a) soil pressures are a function of wall movement, (b) the presence of water in the backfill (pore water) will affect total pressure, and (c) lateral earth pressures can change with time as drainage occurs and pore-water conditions change.

\subsubsection{Wall stiffness effects}

Deformations and wall movements in excavations are a function of soil strength and wall stiffness, with wall stiffness a function of structural rigidity (EI) and the spacing of anchors. Steel sheet-pile and steel soldier beams with timber lagging systems are considered to be flexible tieback wall systems. Secant cylinder pile, continuous concrete slurry wall, and discrete concrete slurry wall systems are considered to be stiff tieback wall systems. The effect of wall stiffness on wall displacements and earth pressures is described in Xanthakos (1991). Often, in practice, trapezoidal (apparent) pressure distributions are used to evaluate flexible tieback wall systems, and triangular pressure distributions are used to evaluate stiff tieback wall systems.

\subsubsection{Relation between earth pressures and wall movements}

With tieback wall construction, the primary ground support system (soldier beams in the case of a discrete wall system, or sheet-pile or slurry concrete in the case of a continuous wall system) is installed before excavation begins. At this point the soil pressures on both sides of the wall are near or at at-rest conditions. As excavation begins, the wall moves toward the excavation, and soil pressures behind the wall decrease. At the same time, the movement toward the excavation increases soil pressures in the unexcavated soils on the front side of the wall. If movements are sufficiently large, limit state conditions are reached, resulting in active pressure on the back side of the wall and passive pressure on the front side of the wall. In general, the amount of movement needed to reach a passive limit state condition is seldom achieved and therefore, typically, the pressure on the front side of the wall increases in the linear elastic range to levels somewhat in excess of at-rest conditions. If movements continue after limit state conditions are reached, the earth pressures will remain constant at a level representing active or passive pressure, as the case may be.

After completing the first-stage excavation (excavation to just below the level of the first tieback anchor), the upper tieback anchor is installed and prestressed to the required level. Active pressure conditions may exist after the prestress load is applied, provided that as excavation progresses the movements of the wall toward the excavation are consistent with active limit state conditions. Higher prestress levels, however, can pull the wall back into the excavation sufficiently to cause soil pressures behind the wall to increase. Depending on the 
level of prestress, soil pressures behind the wall in the region of the tieback anchor can approach or possibly exceed at-rest pressure conditions.

An understanding of the interdependence between wall deformations and earth pressures is fundamental to the proper selection of earth pressures and earth pressure distributions for the design of tieback wall systems. The relationship between the movement of sand backfills and the static earth pressure forces acting on the wall is shown in Figure 1.1. The figure is based on the data from model retaining wall tests conducted by Terzaghi $(1934,1936,1954)$ at the Massachusetts Institute of Technology and tests by Johnson (1953) at Princeton University under the direction of Tschebotarioff. The backfill movements are

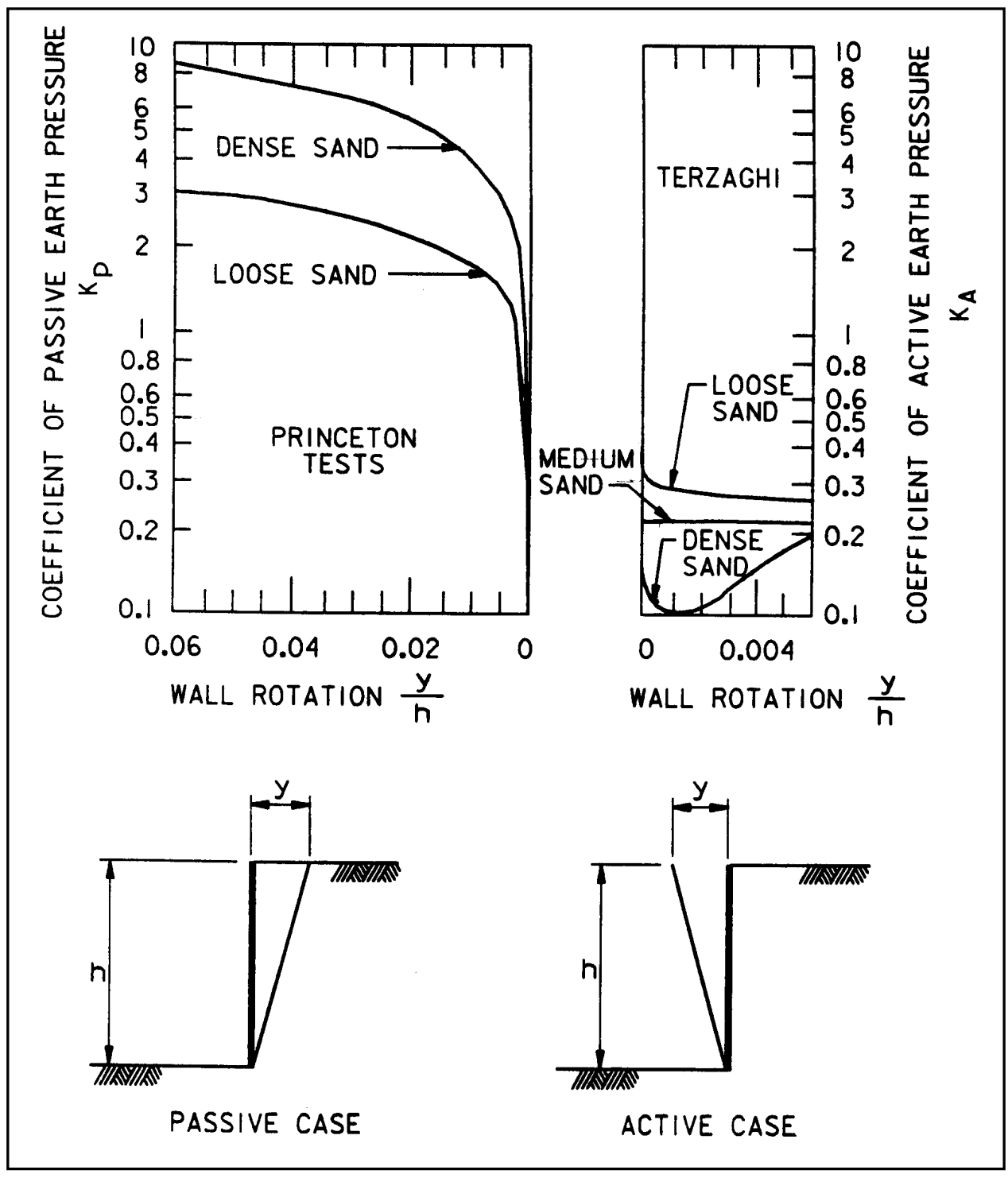

Figure 1.1. Relationship of earth pressure versus wall movements (after NAVFAC DM 7.2, 1982) 
presented as the movement at the top of the wall ( $y$ ) divided by the height of the wall $(H)$, and the earth pressure forces are expressed in terms of an equivalent horizontal earth pressure coefficient $\left(K_{h}\right)$. Variable $K_{h}$ is equal to the horizontal effective stress $\left(\sigma_{h}^{\prime}\right)$ divided by the vertical effective stress $\left(\sigma_{v}^{\prime}\right)$. Other investigations, both experimental and analytical (finite element method), have been made to determine the wall movements needed to reach active and passive limit state conditions. These investigations are summarized in Table 1.1, after Clough and Duncan in Chapter 6 of Fang (1991).

\begin{tabular}{|c|c|c|}
\hline $\begin{array}{l}\text { Table 1.1 } \\
\text { Approximate M } \\
\text { Minimum Activ } \\
\text { (adopted from }\end{array}$ & $\begin{array}{l}\text { f Move } \\
\text { num Pa } \\
\text { Dunca }\end{array}$ & $\begin{array}{l}\text { ed to Reach } \\
\text { ressure Conditions }\end{array}$ \\
\hline & & of $\Delta / H^{1}$ \\
\hline Type of Backfill & Active & Passive \\
\hline Dense sand & 0.001 & 0.01 \\
\hline Medium-dense sand & 0.002 & 0.02 \\
\hline Loose sand & 0.004 & 0.04 \\
\hline Compacted silt & 0.002 & 0.02 \\
\hline Compacted lean clay & $0.010^{2}$ & $0.05^{2}$ \\
\hline Compacted fat clay & $0.010^{2}$ & $0.06^{2}$ \\
\hline $\begin{array}{l}\text { Value of } \Delta \text { is equal } \\
\text { passive pressure by ti } \\
\text { Under stress condit } \\
\text { cohesive soils creep c } \\
\text { only temporarily. With } \\
\text { remains constant, acti } \\
\text { time. }\end{array}$ & $\begin{array}{l}\text { p of wall } \\
\text { Islation; } H \\
\text { minimum c } \\
\text { ovements } \\
\text { ents will cc } \\
\text { increase v }\end{array}$ & $\begin{array}{l}\text { imum active or maximum } \\
\text { assive earth pressures, } \\
\text { e active or passive pressures } \\
\text { emain constant. If movement } \\
\text { pressures will decrease with } \\
\end{array}$ \\
\hline
\end{tabular}

Note that the magnitude of wall movement to fully mobilize the shear resistance in the backfill and thus develop minimum active or maximum passive earth pressure conditions depends upon the soil type. For sands, the required movements are distinguished by soil density. Loose sands require more wall movement than do dense sands for the same wall height. Clough and Duncan (1991) and Duncan, Clough, and Ebeling (1990) give the following easy-toremember guidelines for the amounts of movement required to reach the pressure extremes in a cohesionless soil. The movement required to reach the minimum active condition is no more than about 1 in. $(25 \mathrm{~mm})$ in $20 \mathrm{ft}(6 \mathrm{~m})$ $(\Delta / H=0.004)$, and the movement required to reach the maximum passive condition is no more than about $1 \mathrm{in} .(25 \mathrm{~mm})$ in $2 \mathrm{ft}(0.6 \mathrm{~m})(\Delta / \mathrm{H}=0.04)$.

\subsubsection{Construction short-term, construction long-term, and postconstruction conditions}

During anchor wall construction there is a basic tendency for the wall and soil retained by the anchored wall system to move toward the excavation as excavation proceeds in front of the wall. When these movements are sufficient to fully mobilize the shear resistance within the soil wedge within the retained soil, the backfill is said to be in an active state of stress. The lateral stress exerted, for 
example, by a retained (cohesionless) granular soil on the anchor wall is often expressed at a given depth as

$$
\sigma_{h}{ }^{\prime}=K_{a} \cdot \sigma^{\prime}{ }_{v}
$$

where $K_{a}$ is active earth pressure coefficient and $\sigma^{\prime}{ }_{v}$ is the vertical effective stress. Above the water table, $\sigma^{\prime}{ }_{v}$ is computed as

$$
\sigma_{v}^{\prime}=\gamma_{\text {moist }} \cdot \text { depth }
$$

where $\gamma_{\text {moist }}$ is the moist unit weight of the granular soil. The active earth pressure coefficient $K_{a}$ is expressed in terms of the shear strength of the soil using, for example, Rankine's earth pressure theory or Coulomb's earth pressure theory, depending upon the value for wall-to-soil interface friction. It may be easily shown, using Mohr's circle for a granular soil obeying the Mohr-Coulomb shear strength failure criteria, that the Rankine earth pressure coefficient $K_{a}$ is given by the relationship

$$
K_{a}=\tan ^{2}\left(45^{\circ}-\frac{\phi^{\prime}}{2}\right)
$$

where the Mohr-Coulomb soil shear strength for a cohesionless soil is

$$
\tau_{f}=\sigma_{n}^{\prime} \tan \left(\phi^{\prime}\right)
$$

and where $\sigma_{n}^{\prime}$ is the normal effective stress on the failure plane and $\phi^{\prime}$ is the effective angle of internal friction of the granular soil. For completeness, recall that the general Mohr-Coulomb soil shear strength relationship for a soil is given by

$$
\tau_{f}=c^{\prime}+\sigma_{n}^{\prime} \tan \left(\phi^{\prime}\right)
$$

In the case of the effective cohesion, $c^{\prime} \neq 0$, Equation 1.1 becomes

$$
\sigma_{h}{ }^{\prime}=K_{a} \cdot \sigma^{\prime}{ }_{v}-2 \cdot c^{\prime} \cdot \sqrt{K_{a}}
$$

Equation 1.1 or 1.6 can also be used to compute the horizontal effective stress $\sigma^{\prime}{ }_{h}$ exerted by the soil on the retaining wall for cases in which there is a water table within a free-draining granular soil (and when the wall movements are sufficient to fully mobilize the shear resistance within the soil wedge within the retained soil). However, the value of the vertical effective stress $\sigma_{v}^{\prime}$ is computed by

$$
\sigma_{v}^{\prime}=\sigma_{v}-u
$$


In this partially or fully submerged case, the total horizontal thrust on the back of the wall is equal to the horizontal effective stress $\sigma^{\prime}{ }_{h}$ from Equation 1.6 plus the pore-water pressure, $u$. For a free-draining granular backfill, the porewater pressure used to compute $\sigma_{v}^{\prime}$ in Equation 1.7 is the steady-state pore-water pressure and does not usually include excess pore-water pressures generated in the soil by changes in the total stress regime due to construction activities (excavation, etc.). This is because the rate of construction is much slower that the ability of a pervious and free-draining granular soil site's ability to rapidly dissipate construction-induced excess pore-water pressures.

For sites containing soils of low permeability (soils that drain slower than the rate of excavation/construction), the total pore-water pressures will not have the time to reach a steady-state condition during the construction period. In these types of slow-draining, less permeable soils, the shear strength of the soil during wall construction is often characterized in terms of its undrained shear strength, $S_{u}$.

It should be noted that a water table need not be present in low-permeability soil in order to use this approach of shear strength characterization. Additionally, the value of a soil's undrained shear strength $S_{u}$ may not be a constant but can vary with depth, even for a homogeneous soil site.

These types of slow-draining, less-permeable soils are often referred to as "cohesive soils." The horizontal earth pressures are often computed using values of the undrained shear strength for these types of soils, especially during the short-term, construction loading condition (sometimes designated as the undrained loading condition, where the term undrained pertains to the state within the soil during this stage of loading).

However, as time progresses, walls retained in these types of soils can undergo two other stages of construction loading: the construction long-term (drained or partially drained) condition and the postconstruction/permanent (drained) condition. Under certain circumstances, earth pressures may be computed in poorly drained soils using the Mohr-Coulomb (effective stressbased) shear strength parameter values for the latter load case(s).

Liao and Neff (1990), along with others, point out that all three stages of loading must be considered when designing retaining-wall systems, regardless of soil type. As stated previously, for granular soils, the construction short- and long-term conditions are usually synonymous, since drainage in these soils occurs rapidly. Differences in the construction short- and long-term conditions are generally significant only for "cohesive soils." Liao and Neff (1990) recognized that, for clays, the apparent increase of strut of tieback loads with time can probably, in most cases, be explained in terms of negative pore-water pressure dissipation leading to increases in total stress imposed on the excavation wall. Designers of walls in clay need to carefully consider this issue during the course of the tieback wall design. Changes in the groundwater level (if present) before and after wall construction, as well as for the postconstruction (i.e., permanent) condition, must also be considered. This is usually accomplished for sands and other free-draining soils, for both the short and long term, by effective stress analysis using effective weights (buoyant unit weights) and pore-water 
pressures. For short-term loadings, clay soils are evaluated based on a total stress analysis (i.e., saturated soil weights for submerged soils, moist unit weights for soils above the water table). Internal pore pressures (within the soil mass) are not considered explicitly in total stress analyses, but the effects of the pore pressures for the undrained condition are reflected in the soil's undrained shear strength $\left(S_{u}\right)$. Duncan and Buchignani (1975) observed that, if the laboratory specimens are representative of the soils in the field, the pore pressures in the laboratory specimens will be the same as the pore pressures in the field at the locations where the total stresses are the same, and the use of total stress strength parameters from undrained (shear) tests therefore properly accounts for porepressure effects in short-term, undrained (loading) conditions. For long-term loadings, clay soils are evaluated by effective stress analysis in the same manner as for free-draining soils according to Weatherby (1998).

Designers must work closely with geotechnical engineers to develop a soils testing program that will produce soil strength parameters representative of each condition - the construction short-term, construction long-term, and postconstruction. The program should address both laboratory and field testing requirements.

Some load cases to be evaluated for permanent multi-anchored retaining walls at hydraulic structures can include the presence of a pool of water in the excavated region in front of the anchored wall. For those load cases, external water pressures should be taken into account in the analyses, whether they are performed in terms of total or effective stresses.

\subsection{State of the Practice}

\subsubsection{General}

The determination of anchor loads and wall bending moments requires knowledge about the interaction between the wall and the soil during successive stages of excavation, as well as after completion of the project. Interaction between the wall and soil is difficult to predict. As a result, simplified equivalent beam on rigid support methods of analysis have been developed for use in the design of tieback wall systems.

For flexible tieback wall systems, the simplified method uses apparent pressure diagrams. Probably the most commonly used apparent pressure diagrams are those proposed by Peck (1969) and shown in Figure 1.2 (see also Peck, Hanson, and Thornburn 1974). These apparent pressure diagrams were developed for sands and for clay soils assuming undrained conditions. Anchored walls are seldom built in normally consolidated clay deposits (Weatherby 1998). However, anchored walls are routinely built in overconsolidated clay deposits, and many of these walls have been designed for undrained conditions using the stiff clay apparent pressure diagram $(\gamma H / c \leq 4)$ shown in Figure 1.2.

Peck (1969) stated, "As soon as you think of the apparent pressure as having a total resultant, you depart from the essential concept that the apparent pressure diagram is an envelope. At no cross section in an open cut should the strut loads 
add up to the equivalent of the apparent pressure diagram. One strut may have a total corresponding to the diagram, but if it does, the others should be appreciably less." Peck also stated that "the envelopes, or apparent earth pressure diagrams... were not intended to represent the real distribution of earth

pressure...but instead constituted hypothetical pressures from which there could be calculated strut loads that might be approached but would not be exceeded in the actual cut."

Apparent pressure diagrams, therefore, do not represent the actual distribution of earth pressures along the wall at any particular stage of construction but provide an envelope of earth pressures for all stages of construction. As such, they are often used to determine maximum anchor forces as well as wall bending moments. In the apparent pressure diagram approach, only the final stage of excavation is investigated. The final design usually results in design anchor loads and bending moments that should not be exceeded in the actual cut during or after construction. The designer should be familiar with the assumptions made with respect to the use of apparent pressure diagrams.

Because different movements and earth pressures are encountered with stiff wall systems, a different approach is generally used for their design. Kerr and Tamaro (1990), along with others, noted that the use of apparent pressure diagrams for the design of stiffer wall systems is ill advised.

Active state earth pressure conditions, those usually assumed in the design of tieback wall systems, might not be acceptable when it is necessary to minimize lateral and vertical movements in the ground supported by the tieback wall. Instead, conditions approaching at-rest earth pressures in the soil may be more reasonable for the design of the stiffer tieback wall systems used to minimize ground displacements. 


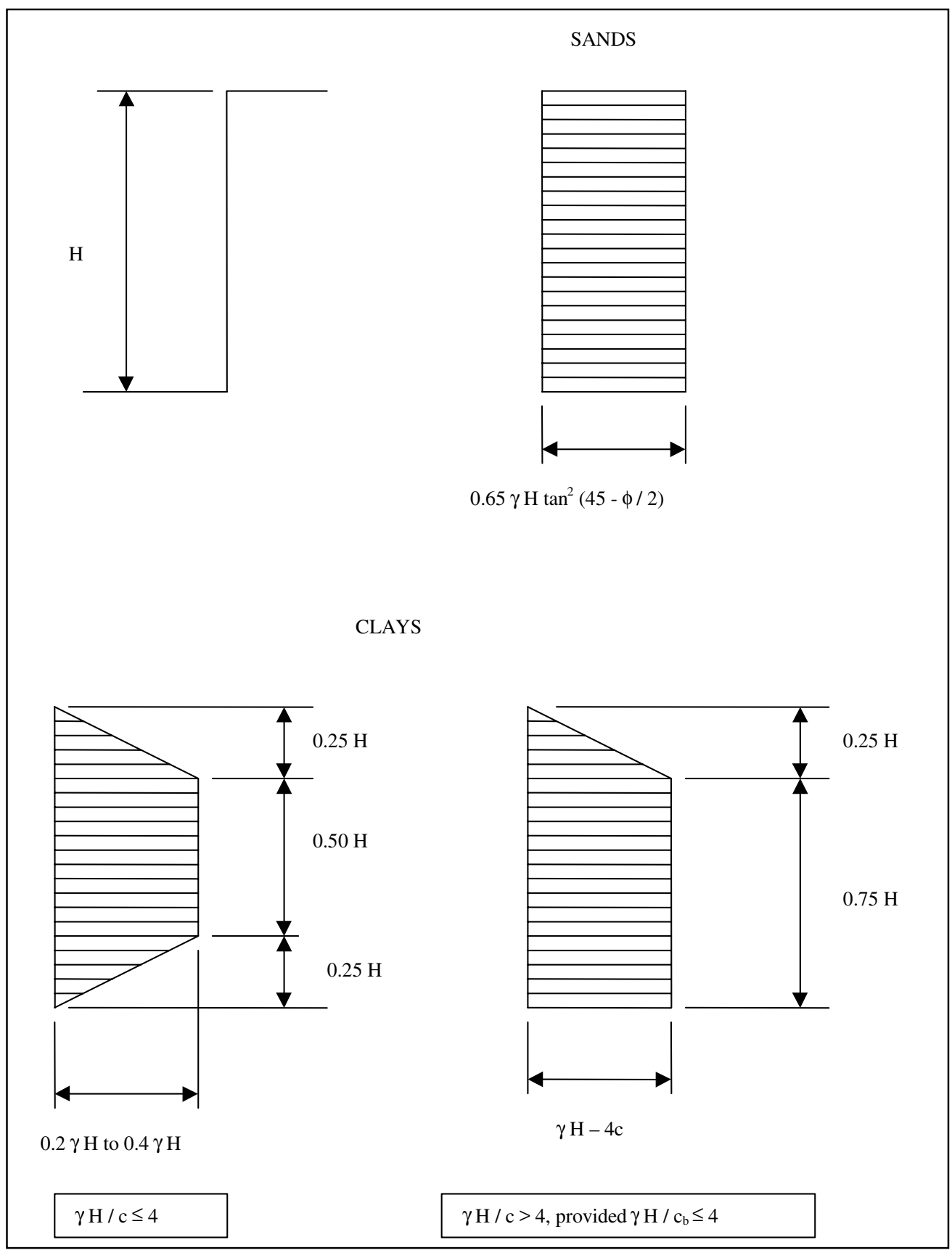

Figure 1.2. Apparent earth pressures diagrams (adapted from Figure 23, FHWASA-99-015; Sabatini, Pass, and Bachus 1999) 


\subsubsection{Flexible tieback wall systems}

The design of ground anchors and structural components of flexible tieback wall systems most often utilizes "apparent" earth pressures diagrams, with the wall idealized as an equivalent beam on rigid supports. It has been observed that the total resultant force obtained from apparent pressure diagrams is somewhere between the active-pressure resultant force and the at-rest pressure resultant force. Long, Weatherby, and Cording (1998) and others note that it is possible to estimate the total apparent pressure force indirectly by multiplying the total active soil pressure force by a factor of safety somewhere between 1.1 and 1.4 (a factor of safety of 1.3 is commonly used for sands).

Alternatively, the factor of safety can be applied to the soil shear strength parameters and the mobilized shear strengths $\left(\mathrm{c}^{\prime}{ }_{\text {mob }}\right.$ and $\left.\phi_{m o b}\right)$ used in a limit equilibrium analysis to estimate the total "apparent" pressure. This "total load" approach permits simple design procedures to be used for walls subject to at-rest pressure conditions, and for various conditions intermediate between active and at-rest pressures. In addition, it allows the "total apparent pressure" to be determined using Rankine, Coulumb, sliding wedge, or limit equilibrium methods. The latter methods provide an opportunity to develop apparent pressures for use in the design of tieback wall systems with irregular ground and layered soil conditions. For the above reasons, the "total load" method has been emphasized in this state-of-the-practice report. The design of flexible wall systems is covered in detail in Azene and Ebeling (2002).

\subsubsection{Stiff tieback wall systems}

Except for the simplest tieback wall systems, the use of apparent pressure (trapezoidal) diagrams for the design of the stiffer wall systems is questionable. With the stiffer wall systems, the apparent pressure diagram approach generally underpredicts the lower tieback loads and the negative moments at the lower supports. Instead, a staged construction analysis using a triangular distribution of lateral soil pressures is often used to design the stiffer wall systems. (A triangular distribution assumes the water table is below base of wall.) The soil pressures usually range from active to at-rest on the driving side and at-rest to passive on the resisting side. The wall is often idealized as a continuous beam on rigid supports, although the use of a continuous beam on nonrigid supports model is becoming more and more the method of choice.

\subsubsection{Common methods of tieback wall analysis}

Four common methods used in the design and evaluation of tieback retaining wall systems are described in Kerr and Tamaro (1990). These were termed

- Equivalent Beam on Rigid Supports Method (RIGID)

- Beam on Elastic Foundation Method (WINKLER)

- Finite Element Method (FEM)

- Limit Analysis (LIMIT)

The first three methods are described in this report. The terminology used is consistent with that used by Kerr and Tamaro, except that the finite element 
method has been separated into two categories-Linear Elastic Finite Element Soil Structure Interaction Method (LEFEM) and Nonlinear Finite Element Soil Structure Interaction Method (NLFEM).

Additional information related to the advantages and disadvantages of the various methods can be found in Kerr and Tamaro (1990) and in succeeding chapters of this report.

\subsubsection{RIGID method}

The simple equivalent beam on rigid supports analysis methods described above are considered versions of the RIGID analysis method. In this method, the soil loads are predetermined and considered to be a following load that is independent of wall displacement. The tieback wall is considered to be a continuous flexural member with stiffness EI. The anchor locations are considered to be supports that prevent lateral translation but permit free beam rotation (hinged supports). The foundation material on the front side of the wall is assumed to act as a fictitious support, preventing lateral translation of the wall. The RIGID analysis method is illustrated in Figure 1.3.

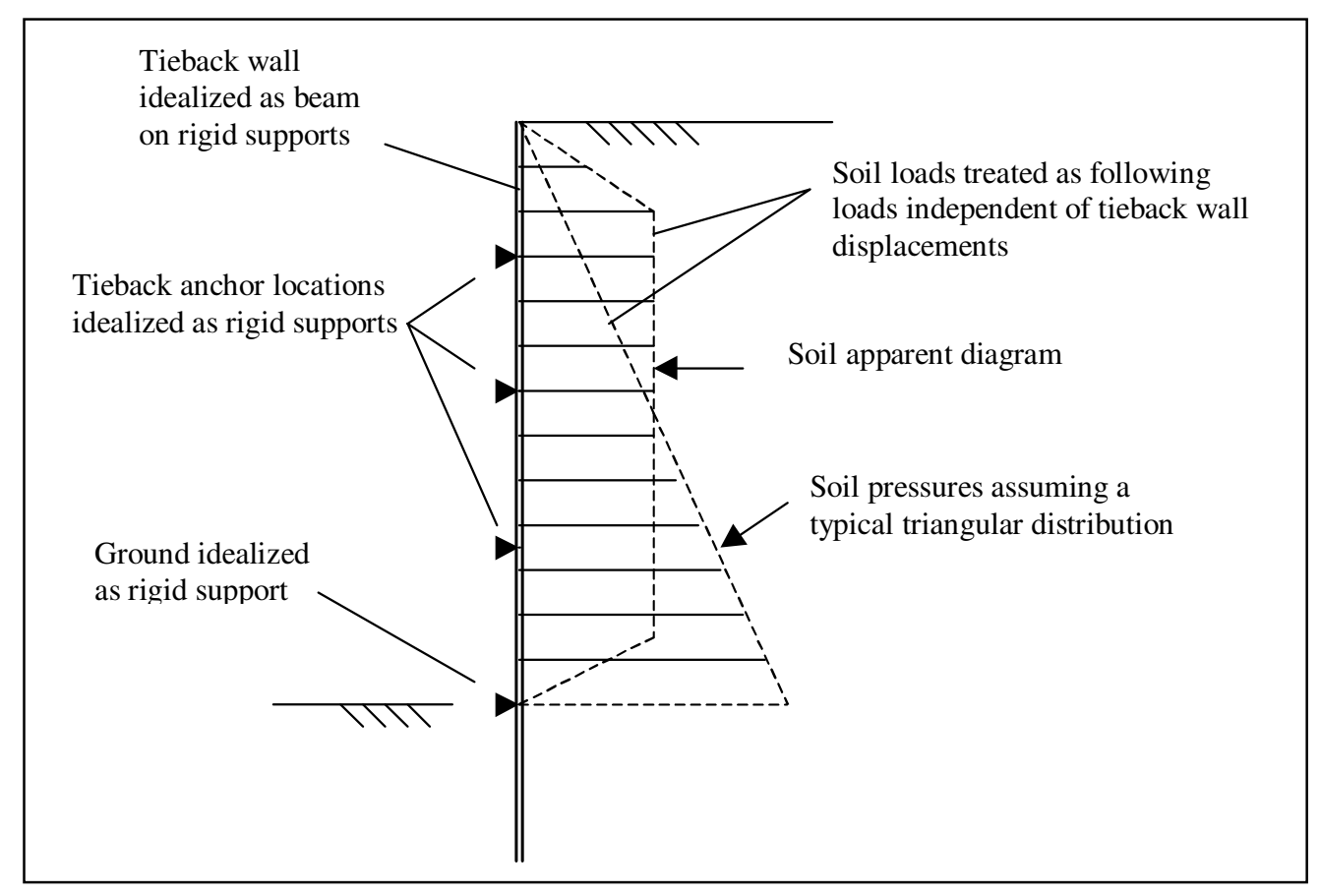

Figure 1.3. Equivalent beam on rigid supports method (RIGID)

\subsubsection{WINKLER method}

The Winkler method is a beam on elastic foundation method of analysis. In its purest form, the tieback wall is considered to be a continuous flexural member with stiffness $E I$ that is supported by a set of infinitely closely spaced soil springs and discrete tieback anchor springs. The soil springs are preloaded to at-rest pressure conditions to represent the condition that exists prior to excavation. As excavation takes place (soil springs on the excavated side removed), the wall 
moves toward the excavation. This movement is the result of the at-rest preload in the soil springs located on the backside of the wall. To keep the system in equilibrium, the soil springs on the excavation side of the wall must increase their loads beyond at-rest. At ground anchor support locations, the tieback is represented by a prestressed (preloaded) tieback anchor spring that also contributes to system equilibrium. The soil springs can be linear elastic or elastoplastic with an elastic stiffness, $k$. The Winkler analysis method (illustrated as Figure 1.4) can be used in a staged excavation analysis or as a final analysis where the completed structure is "wished" into place without consideration of system displacements that occurred during each stage of construction. The Winkler analysis is described in greater detail in Chapter 6.

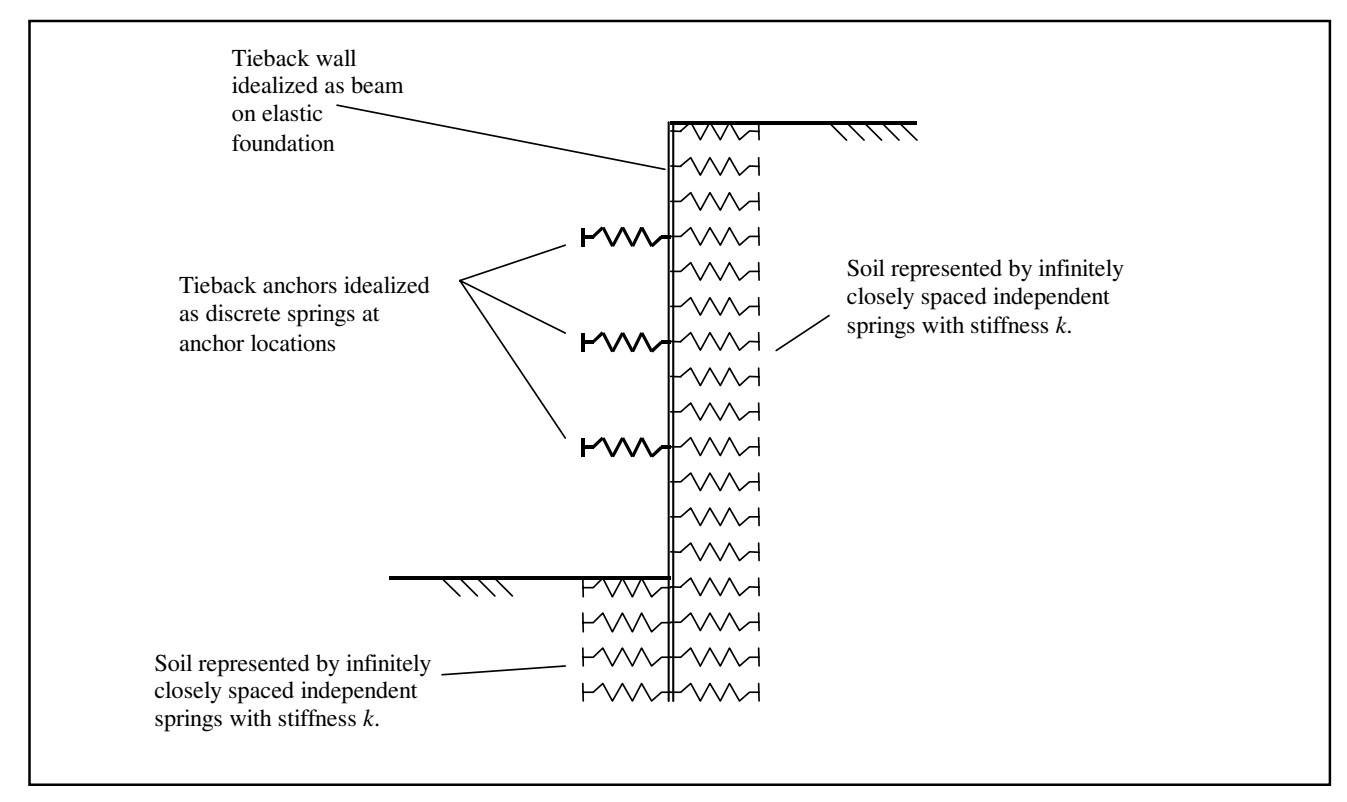

Figure 1.4. Beam on elastic foundation method (Winkler)

\subsubsection{LEFEM and NLFEM analyses}

Linear elastic finite element methods (LEFEM) can be used in a staged excavation analysis and in a loss of anchorage analysis to evaluate anchor loads and bending moment demands in diaphragm walls.

Application of the LEFEM to the analysis of the diaphragm walls of Boston's Central Artery/Third Harbor Tunnel project is described in the American Society of Civil Engineers (ASCE) publication authored by members of the Structural Engineering Institute's (SEI) Technical Committee on Performance of Structures During Construction (ASCE/SEI 2000). One of the types of analysis described in this publication involves the use of linear finite elements to model the soil with constant Young's Modulus values assigned to each of several soil strata used to characterize the site. The two-dimensional, finite element program SOILSTRUCT was used to generate the loads on the wall through an incremental excavation method of analysis. The wall was analyzed for the short-term loading case, which involves incremental excavation/construction stages in fine-grained soils and undrained soil behavior. 
Another analytical approach described in this SEI publication involves the use of linear finite elements to model the diaphragm wall and linear Winkler springs on the excavation side of the wall (with an initial stress equal to at-rest soil pressure). On the active (unexcavated) side, the pressures on the wall are applied through distributed loads. The soil active pressure is assumed to be unchanged throughout the entire sequence of excavation. A finite element mesh is used for the diaphragm wall to capture plate-bending effects for staged excavation analysis, and to capture anchor load redistribution effects for loss of anchorage analyses. Figure 1.5 illustrates the LEFEM with respect to a staged excavation analysis. Additional information on this type of analysis can be found in ASCE/SEI (2000).

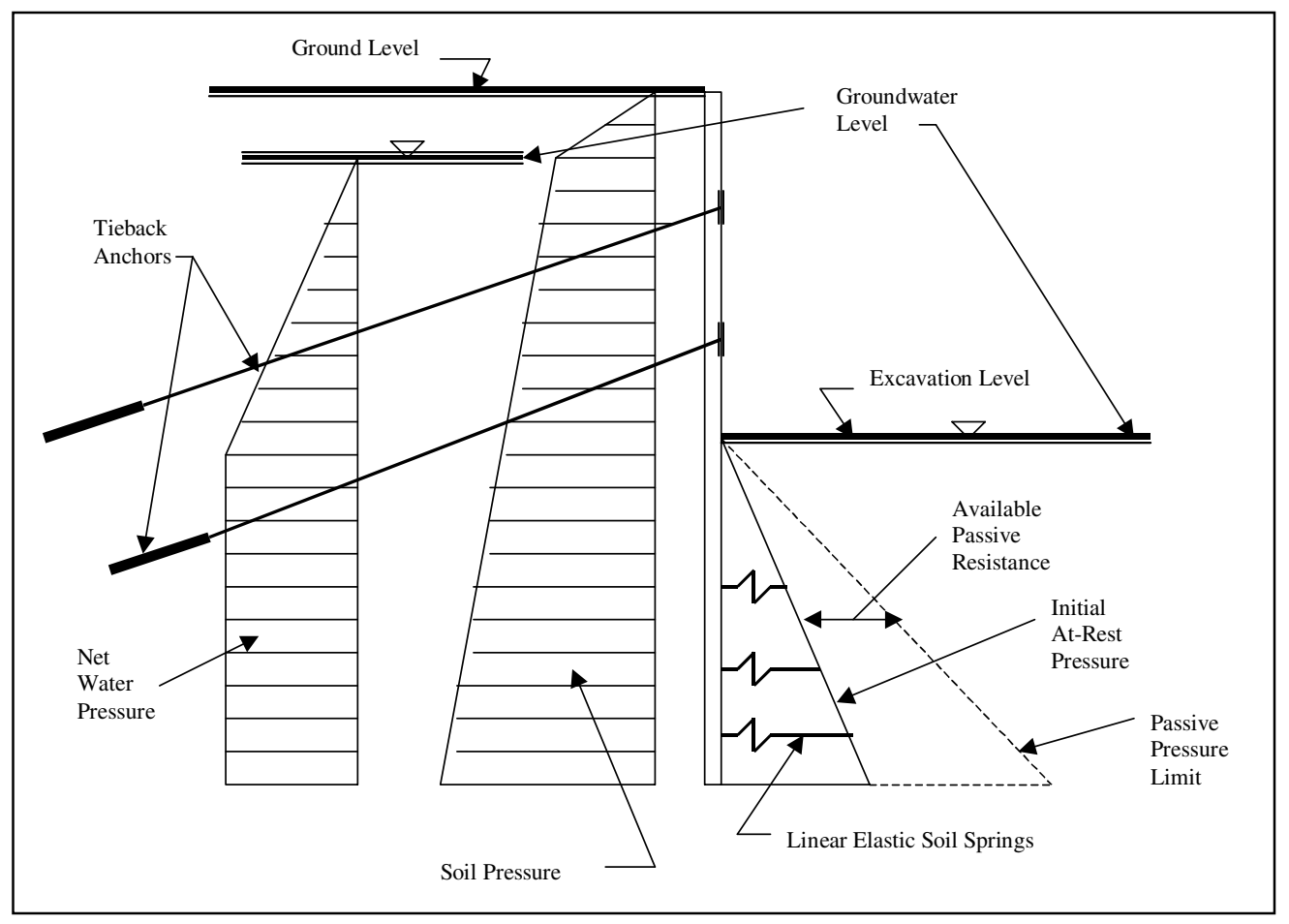

Figure 1.5. Linear elastic finite element model (LEFEM) of diaphragm wall in combination with linear Winkler soil springs

Nonlinear finite element analyses are used in staged construction analyses to capture soil-structure interaction effects. In these analyses, soil nonlinearities can be evaluated and soil pressures can be allowed to vary as a result of structural and related soil deformations. These types of analyses are usually performed using SOILSTRUCT (Ebeling, Peters, and Clough 1992) and SOILSTRUCTALPHA (Ebeling, Duncan, and Clough 1990). Figure 1.6 illustrates the NLFEM with respect to a staged construction analysis of a tieback diaphragm wall. Additional information on this type of analysis with respect to tieback diaphragm wall systems can be found in Chapter 7 of this report, in ASCE/SEI (2000), and in Mosher and Knowles (1990). 


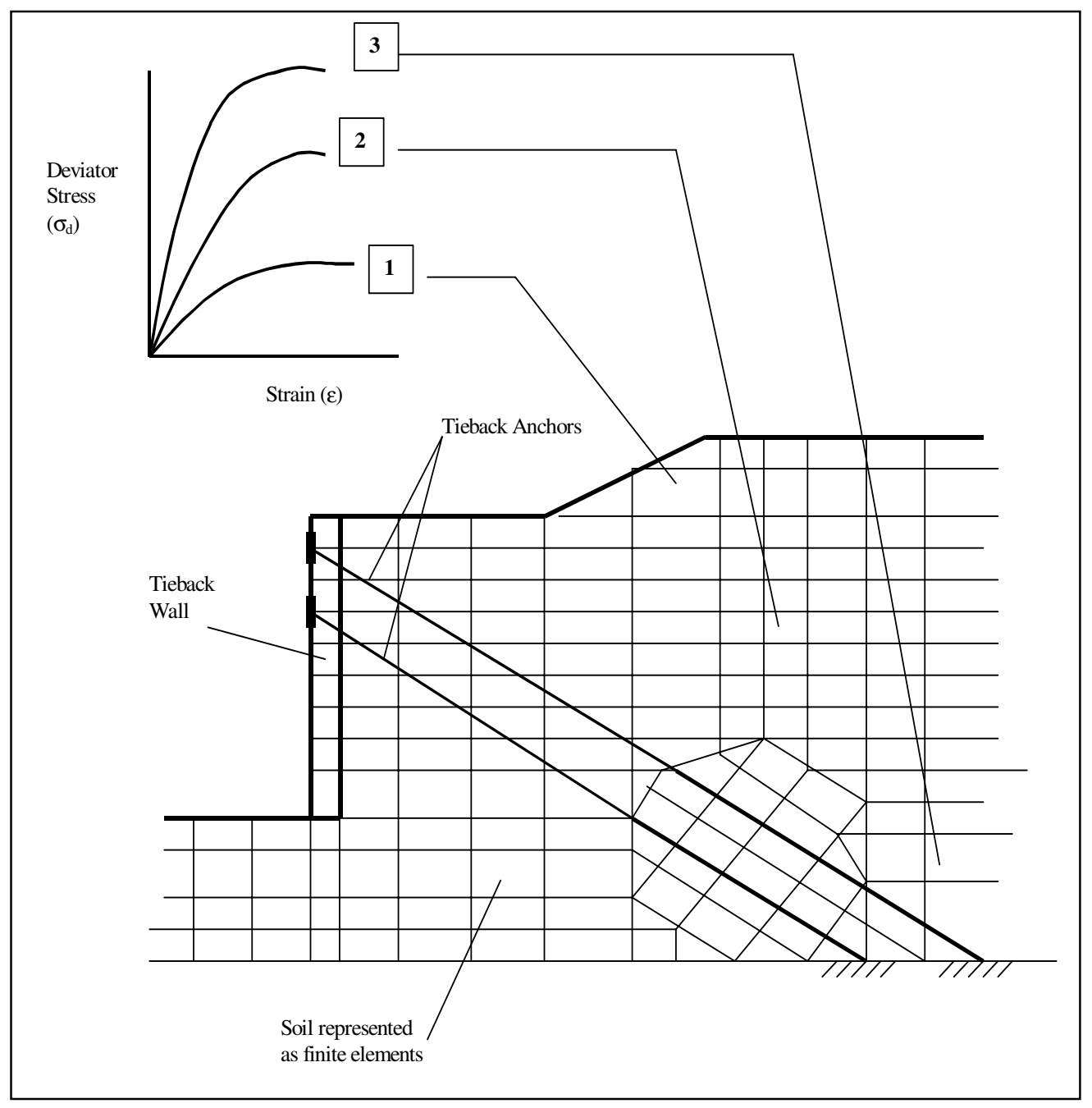

Figure 1.6. Nonlinear finite element method (NLFEM)

\subsubsection{Comprehensive analysis}

When commonly used simple design approaches (RIGID analyses) are used in combination with Winkler spring and/or nonlinear finite element soil-structure analysis procedures, the resulting design will generally meet project performance objectives.

Winkler spring analysis, however, has not developed to the state where it can be used reliably to predict wall and ground displacements. To do so, the effects of construction sequencing must be considered in the analysis and improved methods for determining soil spring load-displacement characteristics developed. At this point, the report addresses only the state of the practice as it applies to Winkler spring-type analyses. Future research by the Corps will be directed toward improving Winkler spring analyses so as to provide a simple evaluation method that can be used to predict displacements as well as ground anchor loads and wall axial loads, moments, and shears. 
Nonlinear finite element soil-structure interaction analyses have been used successfully to provide a detailed and accurate representation of the response of tieback wall systems to all loads encountered during and after construction. The results of nonlinear finite element soil-structure analyses in terms of deflections, earth pressures, wall bending moments and shears, ground anchor loads, ground surface movements, and soil stresses have been shown to agree well with information obtained from site instrument data interpretation. The nonlinear finite element soil-structure interaction method is described in this state-of-thepractice report. 


\section{Tieback Wall Systems}

\subsection{Introduction}

The tieback wall systems selected for consideration in this report are listed below.

- Vertical sheet-pile system with wales and post-tensioned (P-T) tieback anchors.

- Soldier beam system with wood lagging, P-T tieback anchors, and permanent concrete facing.

- Secant cylinder pile system with P-T tieback anchors.

- Continuous reinforced concrete slurry wall system with P-T tieback anchors.

- Discrete concrete slurry wall system (soldier beams with concrete lagging) with P-T tieback anchors.

The various types of tieback systems are described in the following paragraphs. Typical horizontal sections are used to describe each type of tieback wall system (see Figures 2.1-2.5). The P-T anchors used to support these systems are described in Chapter 8 . 


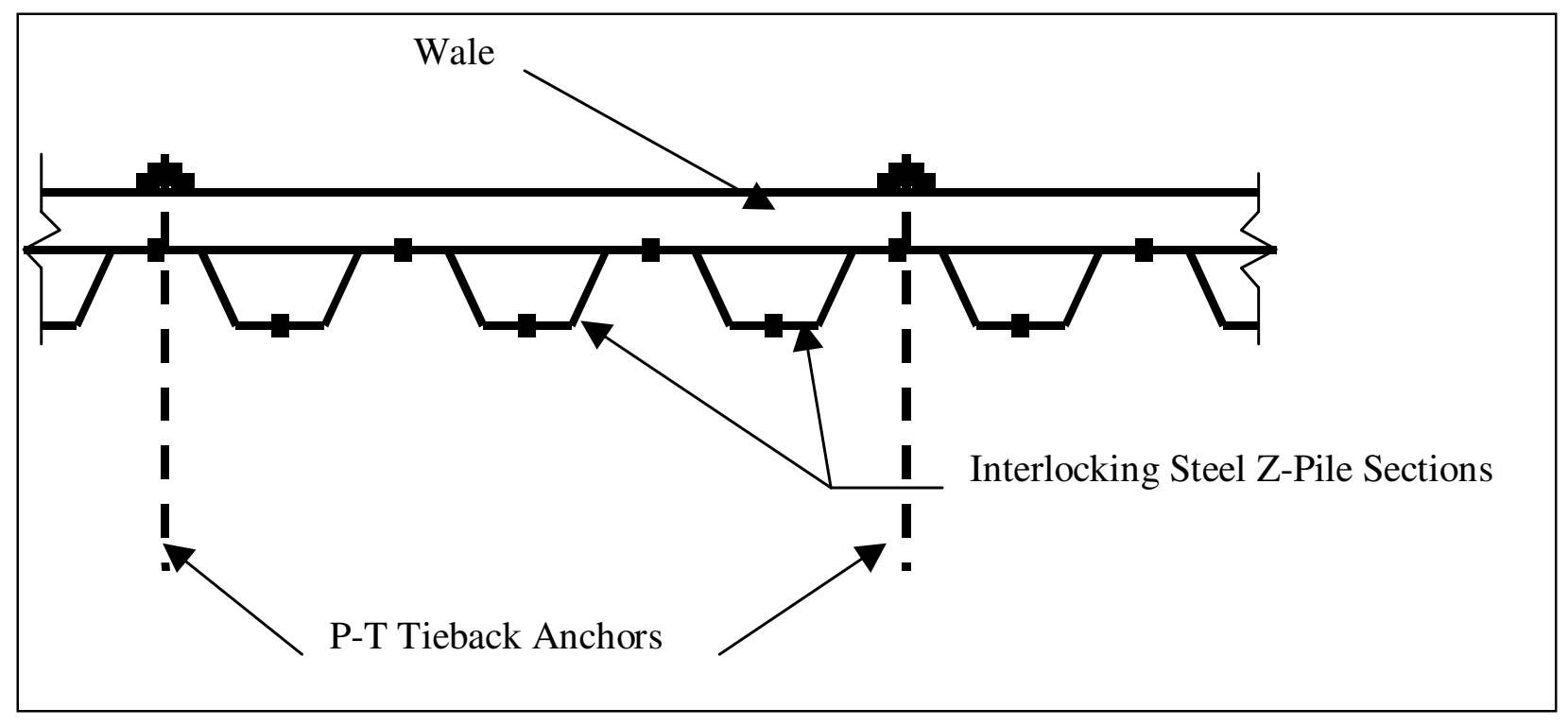

Figure 2.1. Vertical sheet-pile system with wales and post-tensioned tieback anchors

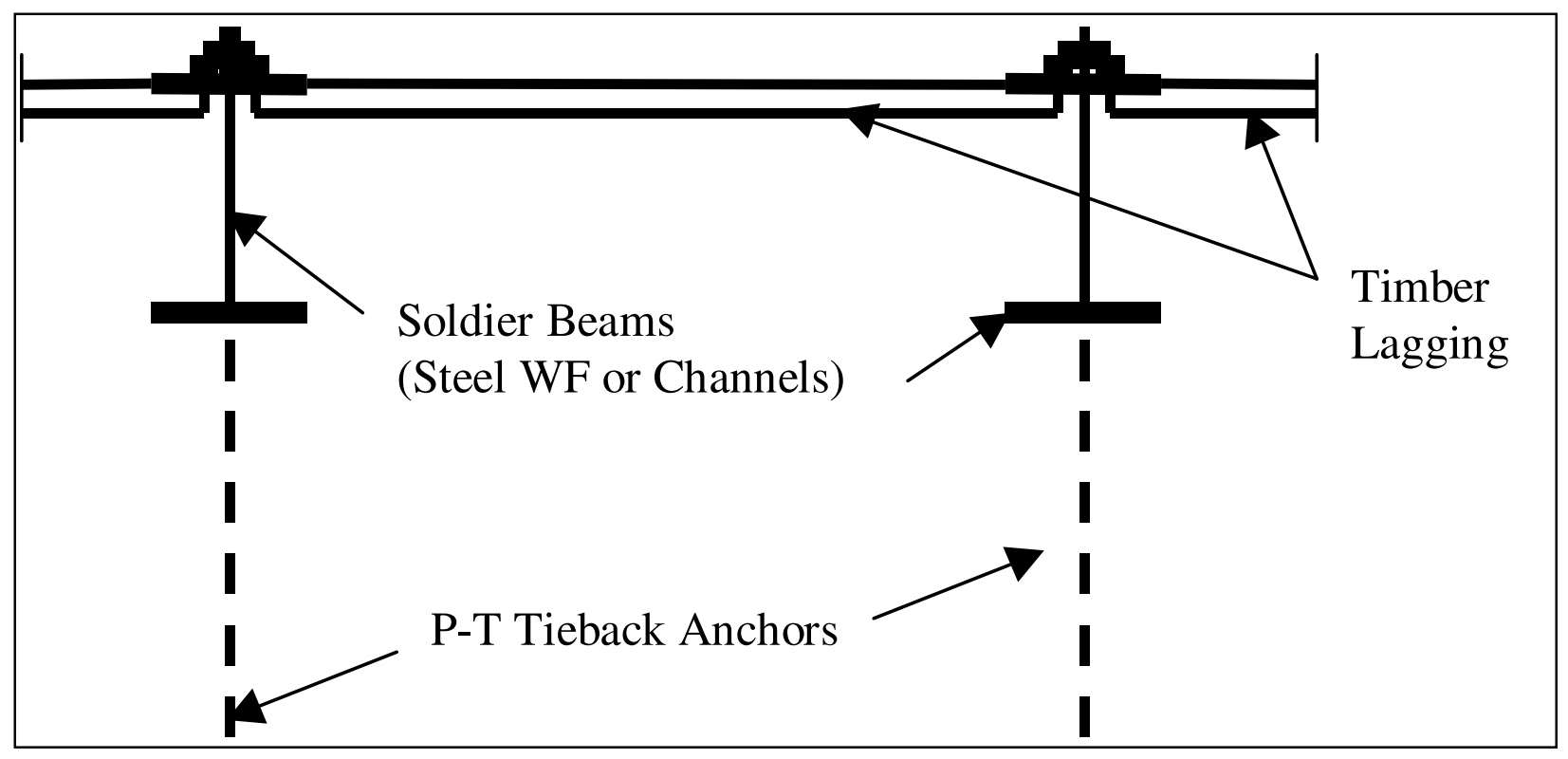

Figure 2.2. Soldier beam system with timber lagging and post-tensioned tieback anchors 


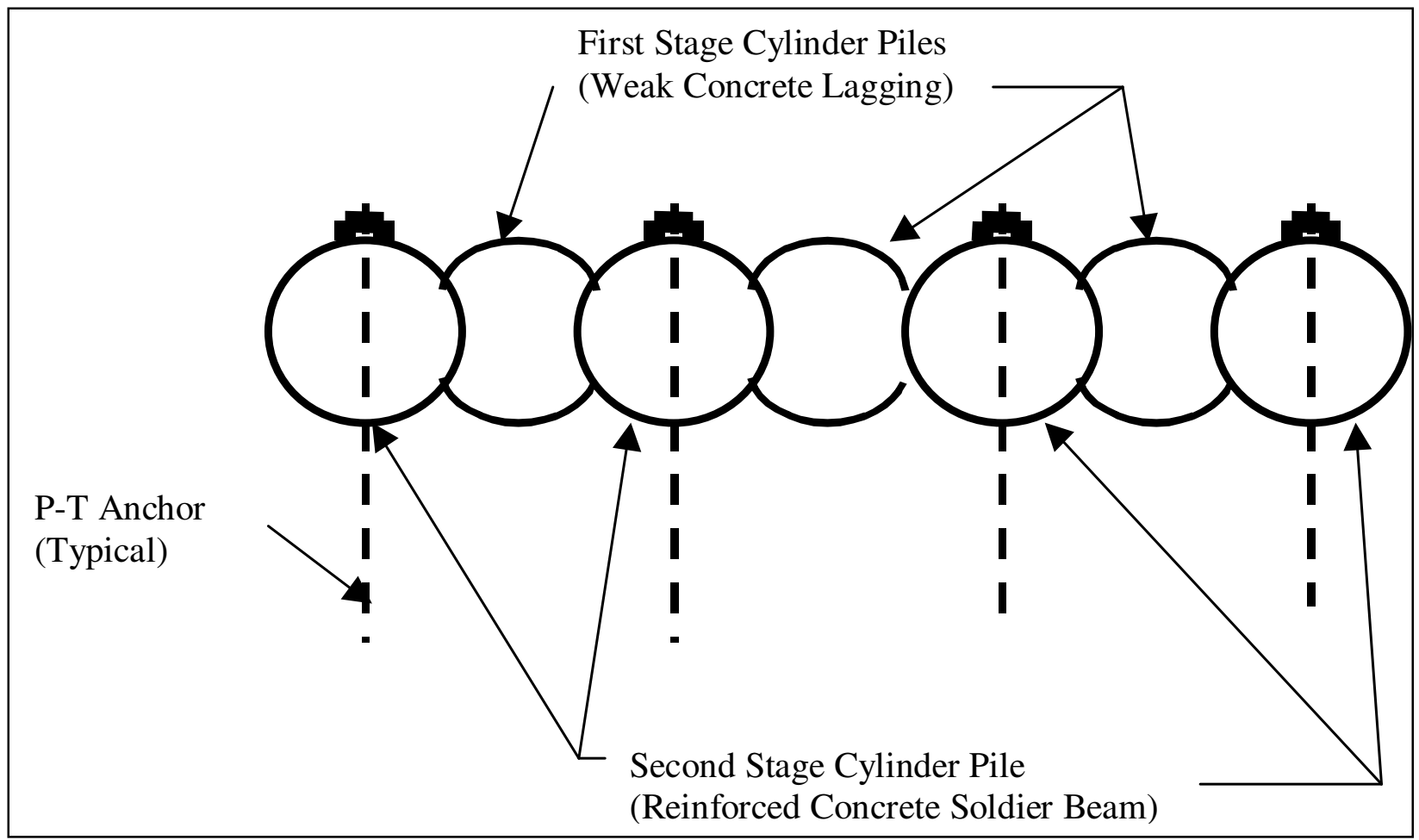

Figure 2.3. Secant cylinder pile system with post-tensioned tieback anchors

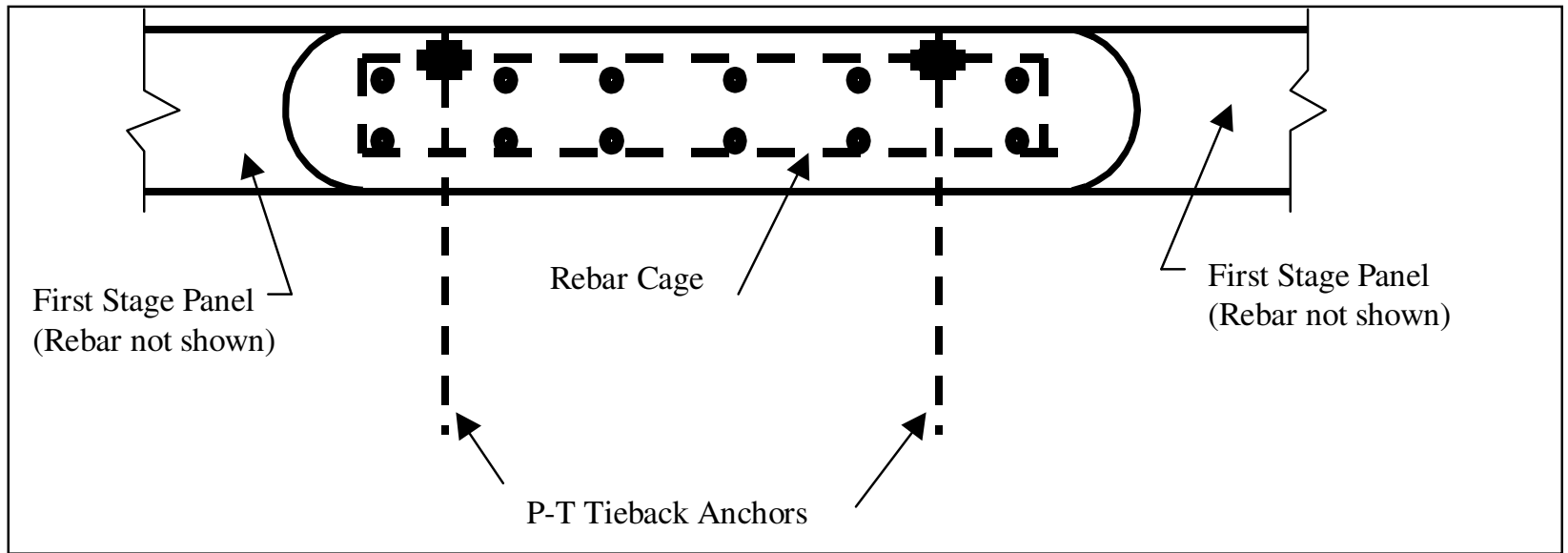

Figure 2.4. Typical continuous concrete slurry wall system with post-tensioned tieback anchors 


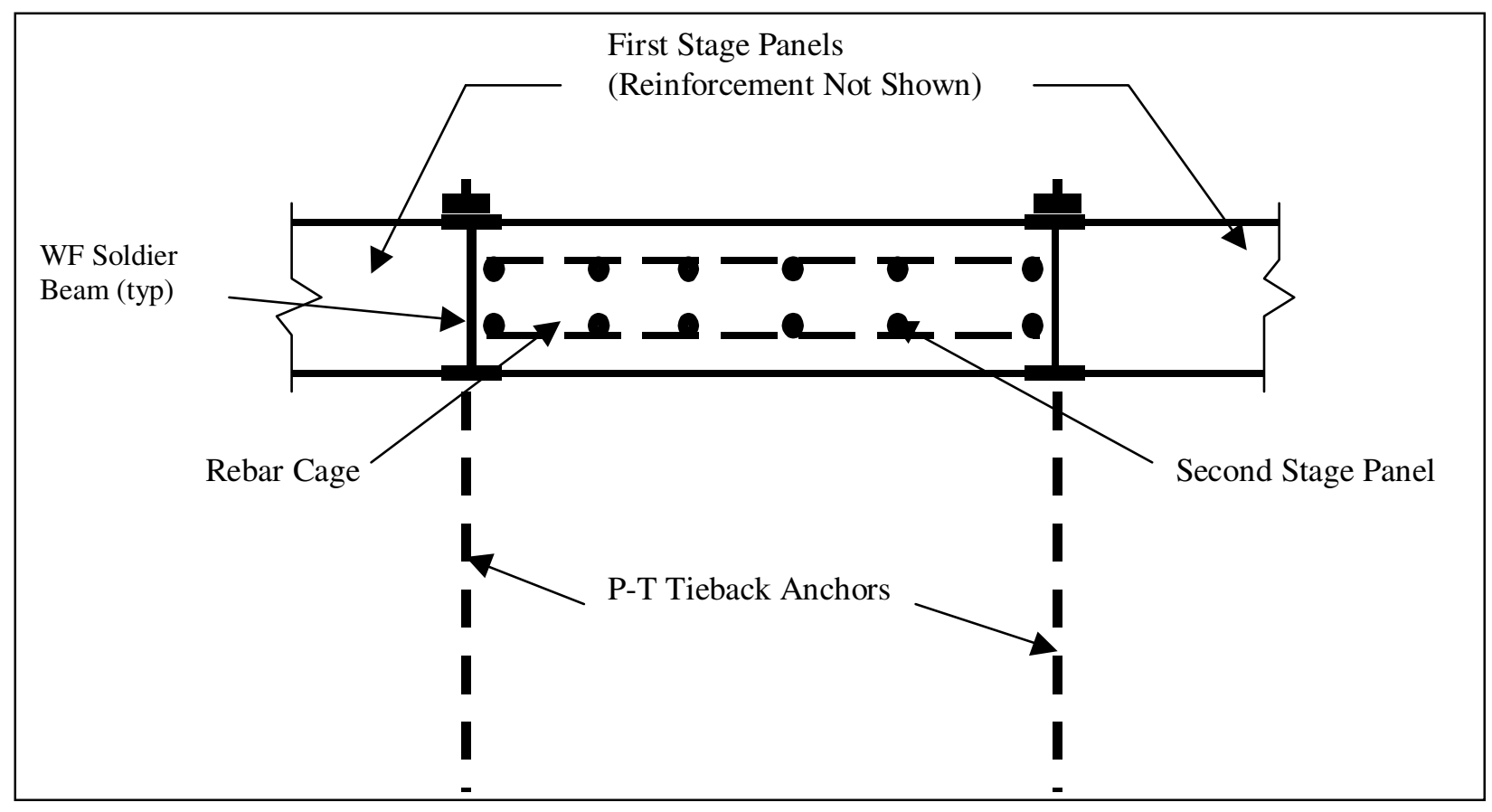

Figure 2.5. Typical soldier beam tremie concrete wall system (soldier beams with concrete lagging) with post-tensioned tieback anchors

\subsection{Vertical Sheet-Pile System with Wales and Post-Tensioned Tieback Anchors}

Vertical sheet-pile systems (Figure 2.1) are usually constructed using interlocking Z-type heavy-gauge steel piling. The piles are either hot-rolled or cold-formed conforming to the requirements of the American Society for Testing and Materials' Standards A328, A572, or A690, with piling conforming to A 328 suitable for most installations. The U.S. Army Corps of Engineers typically uses interlocking Z-type heavy-gauge steel piling for retaining and floodwall applications. Heavy-gauge steel piling is best suited to applications involving potentially squeezing and running soils, such as soft clays and cohesionless silt or loose sand below the water table. Although the interlocks are not completely impervious, sheet-pile systems can reduce seepage and control piping. Sheetpiling used in tieback wall construction will typically be installed by driving with traditional pile-driving equipment. Steel sheetpiling is not suited for use in compact granular soils, where the rock surface is above the desired excavation depth, or in soils that contain cobbles and boulders. Steel sheetpiling is designed to carry earth and seepage pressures by spanning vertically between horizontal wales. The wales span horizontally between tieback anchors. A typical vertical sheet-pile system with wales and P-T tieback anchors is shown in Figure 2.6. 


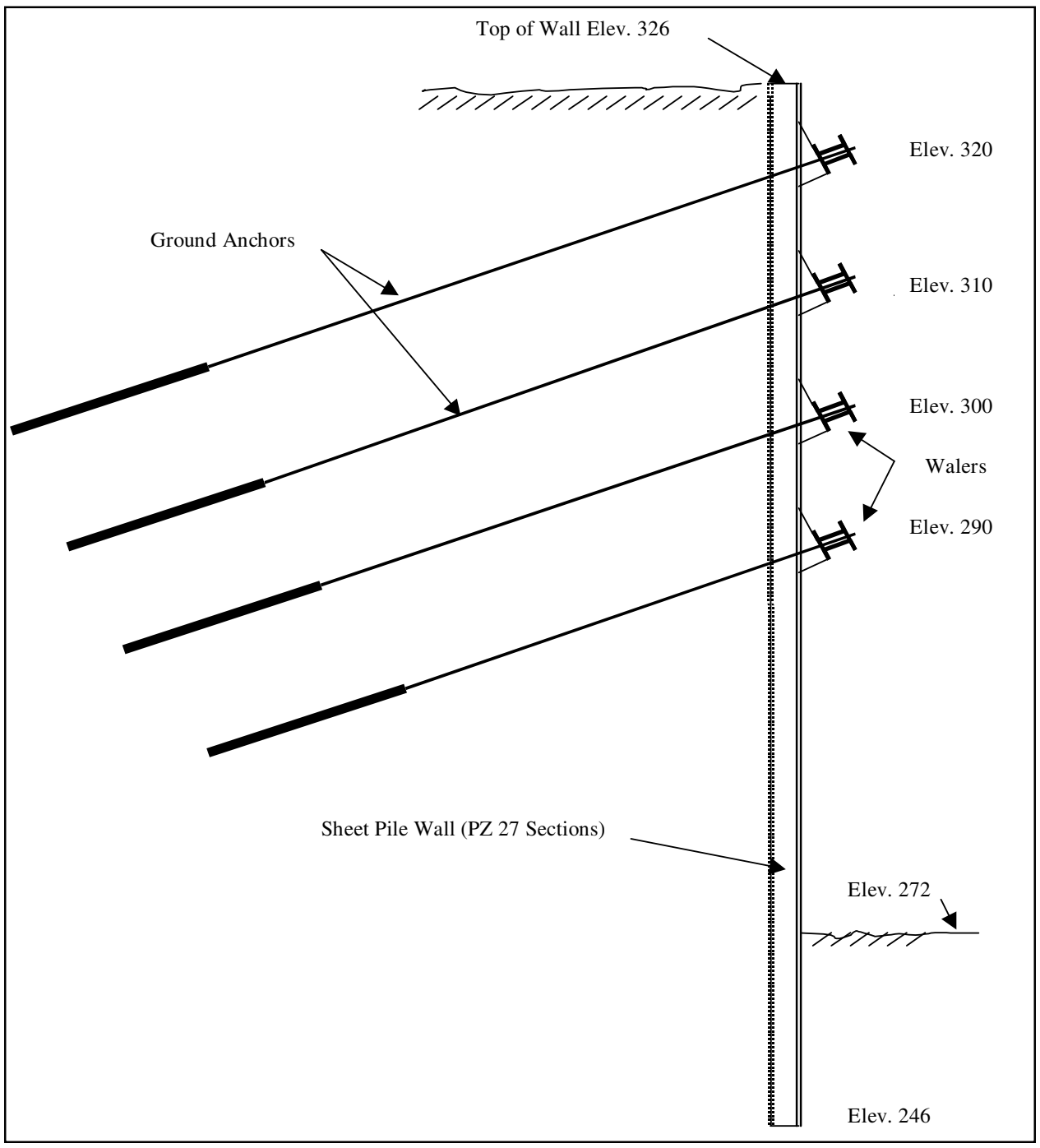

Figure 2.6. Vertical sheet-pile system with post-tensioned tieback anchors (per Olmsted prototype wall)

\subsection{Soldier Beam System with Wood Lagging and Post-Tensioned Tieback Anchors}

The most common form of tieback wall construction involves steel soldier beams with wood lagging and P-T tieback anchors (Figure 2.2). Soldier beams (or soldier piles) can be driven or drilled to the desired elevation. The most common soldier beams are rolled wide-flange (WF) sections or H-pile sections. These types of soldier beams can be driven through compact soil strata and strata containing boulders and cobbles that would otherwise prohibit the use of driven sheetpiling. Soldier beams can also be drilled in. Drilling permits the use of deep, high-capacity WF sections and back-to-back channel sections as soldier beams. Lagging is commonly timber, but precast concrete lagging can also be used. Permanent tieback wall systems that use timber lagging for temporary support often have precast concrete facing or a cast-in-place (CIP) facing system that is installed after the excavation has reached the desired depth. Special trumpet 
sections are generally fabricated into the web of WF and H-pile soldier beam sections to accommodate the tieback anchors, especially in cases where it is undesirable to have walers encroaching on the excavated side of the wall. The purpose of the trumpet is to ensure that tieback loads are concentric to the axis of the pile section. A typical soldier beam system with wood lagging and P-T tieback anchors is shown as Figure 2.7.

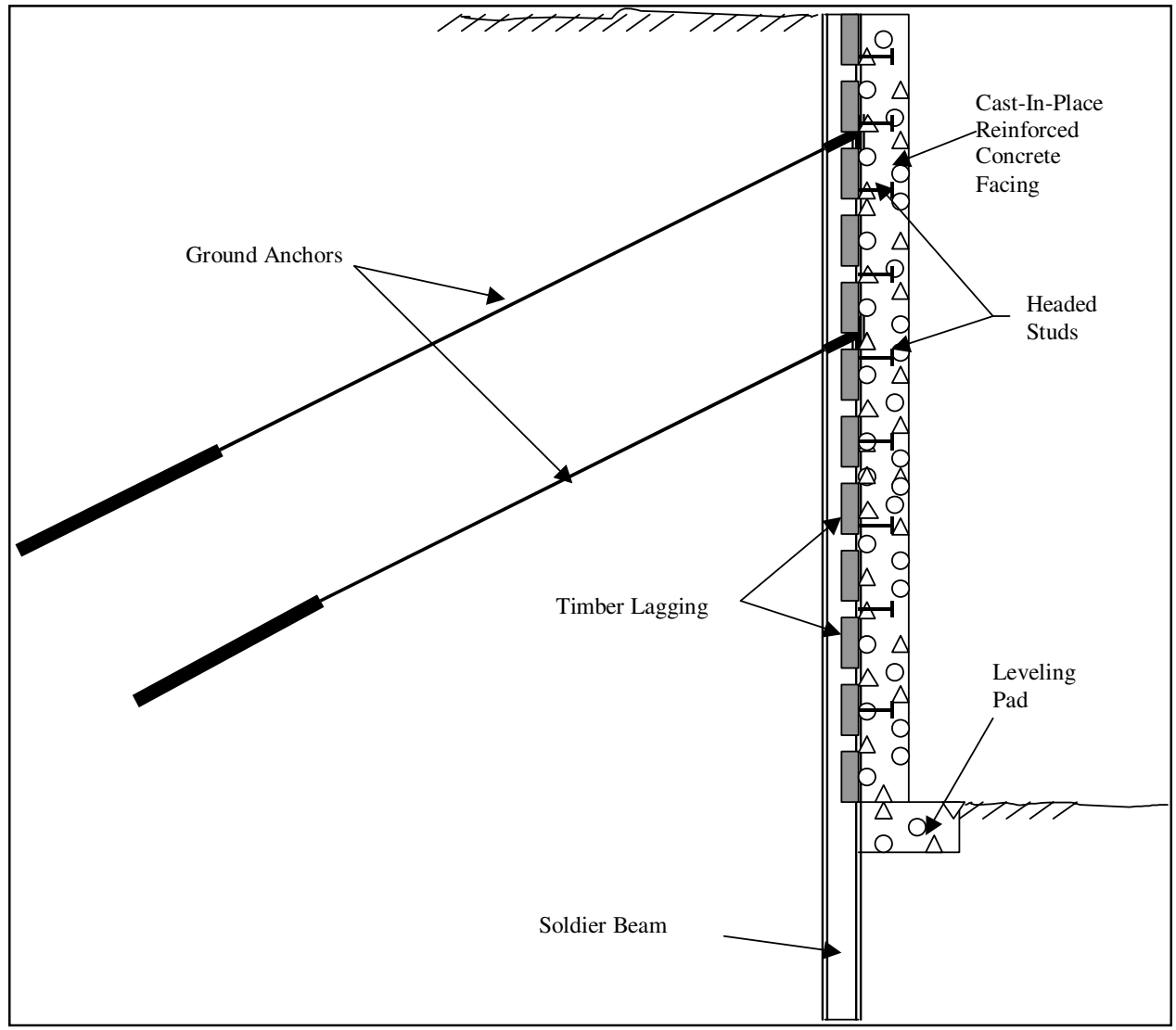

Figure 2.7. Soldier beam system with wood lagging and post-tensioned tieback anchors (general)

\subsection{Secant Cylinder Pile System with Post- Tensioned Tieback Anchors}

The secant pile system with P-T tieback anchors (Figure 2.3) is a type of cylinder pile system in which the piles overlap slightly. The cylinder piles (soldier beams) generally range from 0.6 to $1.2 \mathrm{~m}$ ( 2 to $4 \mathrm{ft}$ ) in diameter. In the secant pile system the lagging piles are drilled first. This is accomplished by using a casing to support the soil, by using slurry mud to stabilize the hole, or with neither casing nor slurry mud in competent materials that will not cave in during construction. Weak concrete is then placed in the hole, and the casing (if a casing is used) is withdrawn. In the second stage, the soldier beam cylinder piles are drilled using a casing equipped with a cutting edge. The cutting edge cuts through the soil strata and a small segment of the weak concrete cylinder pile lagging. Cylindrical reinforcing bar cages, with anchor trumpets wired in place, 
are dropped into and positioned in the second-stage holes. Structural concrete is then placed, by tremie methods if water or mud slurry is present, and the casing (if a casing is used) is withdrawn. Excavation of the wall and installation of tieback anchors begins, once the concrete has reached its required strength. A typical secant cylinder pile system is shown as Figure 2.8.

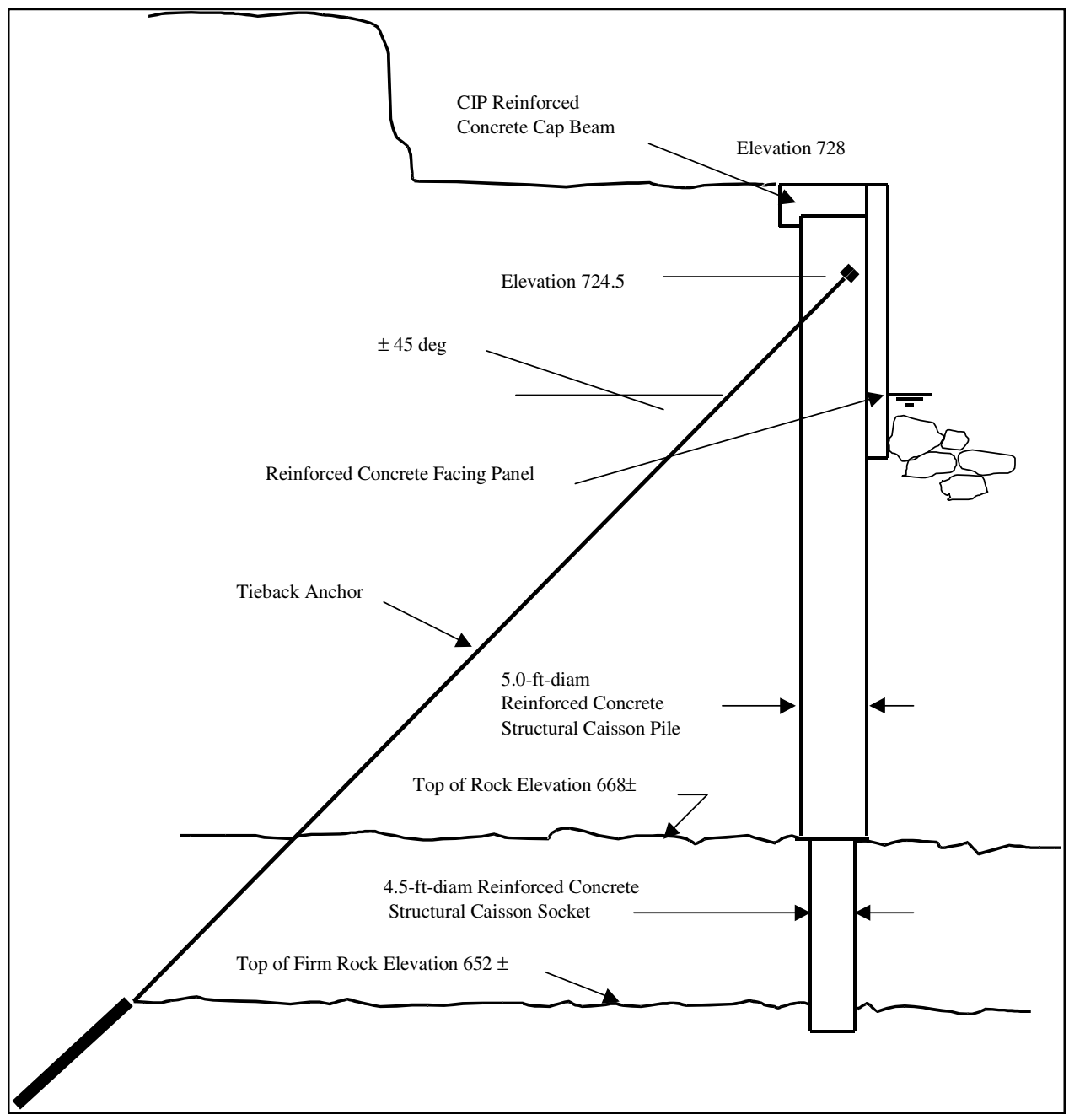

Figure 2.8. Secant cylinder pile system with post-tensioned tieback anchors (Monongahela River Locks and Dams 2 left abutment tieback retaining wall)

\subsection{Continuous Reinforced Concrete Slurry Wall System with Post-Tensioned Tieback Anchors}

A continuous reinforced concrete slurry wall system with P-T tieback anchors is illustrated in Figure 2.4. Slurry wall systems refer to wall systems that use segmental trench excavation stabilized by mud slurry (usually bentonite). The excavated trench acts as formwork for a reinforced concrete tieback wall. Concrete placed by tremie methods displaces the mud slurry and leaves a 
structural concrete wall that can be excavated and tied back in much the same manner as other tieback wall systems. The walls are reinforced like conventionally reinforced cast-in-place concrete walls, except that the reinforcement is installed in preassembled cages that are dropped into the slurry trench just before concrete placement. Slurry wall systems generally range from 0.6 to $0.9 \mathrm{~m}$ ( 2 to $3 \mathrm{ft})$ in thickness and can be placed to depths of $30 \mathrm{~m}(100 \mathrm{ft})$ or more. They can provide a watertight barrier when necessary. They are capable of high moment and shear capacities and provide system redundancy where progressive failure due to loss of anchorage is a concern. Although the construction process can have many variations, it typically can be described as follows (refer to Figure 2.9):

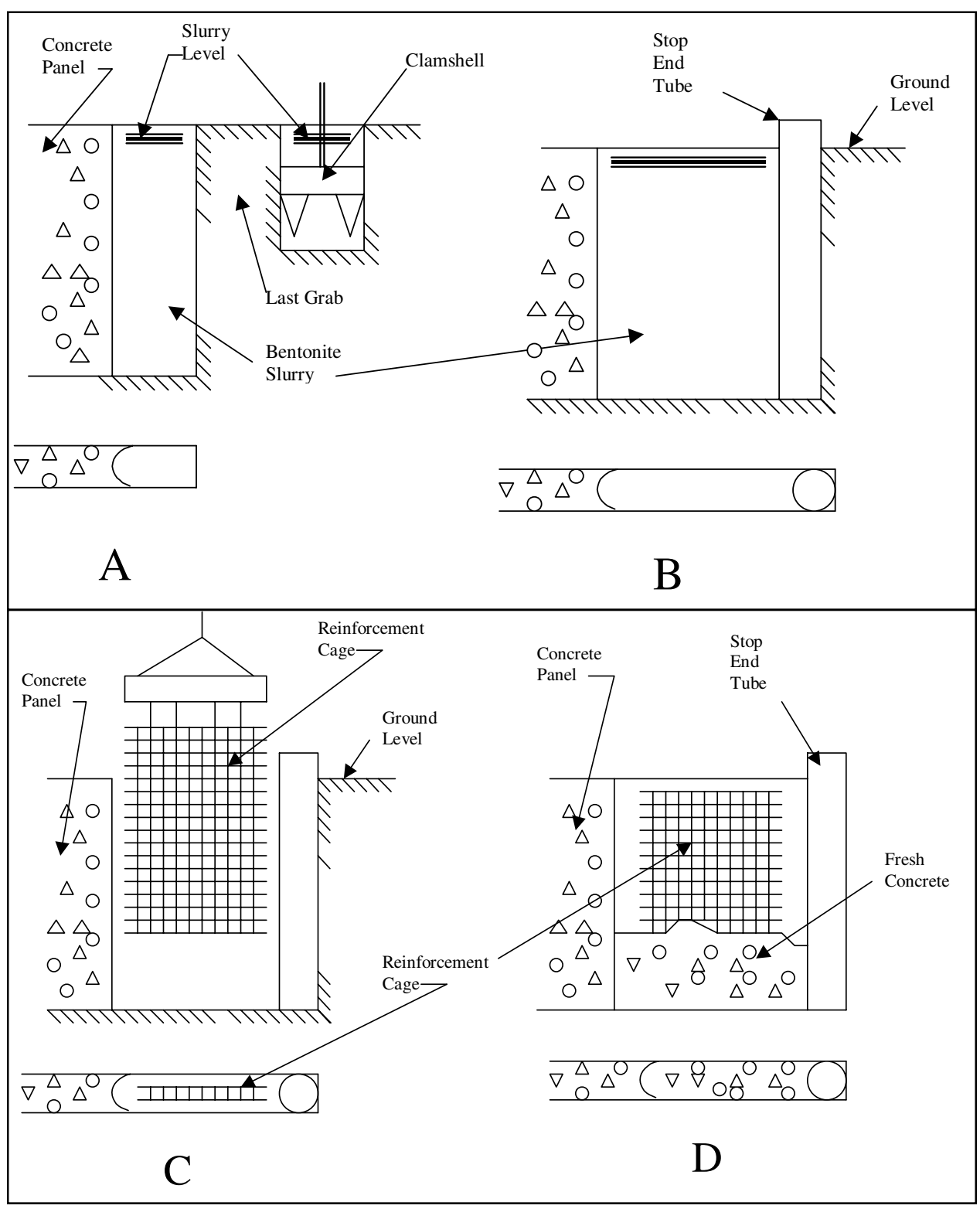

Figure 2.9. Construction sequencing for concrete slurry wall system 
a. Guide walls are constructed at the surface of the excavation for controlling the alignment of the "clamshell bucket" used to excavate a trench panel section. As the panel excavation proceeds downward, the trench is stabilized by mud slurry. This slurry, kept at a level a few feet above the water table and having a unit weight somewhat greater than water, provides positive hydrostatic pressure on the walls of the panel. Panels are excavated in a staggered sequence, with every other panel section excavated as part of first-stage excavation and the remaining alternate panels excavated as part of a second-stage excavation.

b. Stop end tubes, placed as first-stage panel excavation progresses (or drilled in place before panel excavation begins), form guides for the second-stage excavation. These guides, usually cylindrical steel pipes, are pulled as first-stage concrete placement takes place to form a semicircular shear key between first- and second-stage concrete placements.

c. Once the panel has been excavated to its designated depth and the slurry cleaned of all fine excavation material (desanded), the reinforcement cage is lowered into the excavated panel slot and dogged off into place.

d. Tremie pipes (one or more, as required to place the concrete without contamination from the slurry) are lowered into the trenched panel, and the concrete is placed in accordance with acceptable tremie concrete placement standards.

e. After the concrete has reached its desired strength, the excavation and tieback installation construction sequencing process can begin.

Although this construction method provides a competent structural wall system, it should be recognized that the final wall will not be to the standards and tolerances usually associated with formed wall systems. Most often, engineers will assume a 15-percent reduction in concrete strength. They will also assume that, because the reinforcing steel will have a light mud coating that reduces bond, reinforcing bar splices should be 1.5 to 2.0 times those required by ACI 318 (American Concrete Institute, "Building Code Requirements for Structural Concrete and Commentary"). The concrete finish obtained as a result of slurry trench construction methods may not be as desired visually with respect to the exposed sections of the wall. In such cases, cast-in-place concrete or precast concrete panel finishes are usually applied over the exposed surfaces of the slurry wall. Details of the continuous reinforced concrete slurry wall system used in the construction of the new navigation lock at Bonneville Dam are illustrated in Figure 2.10. 


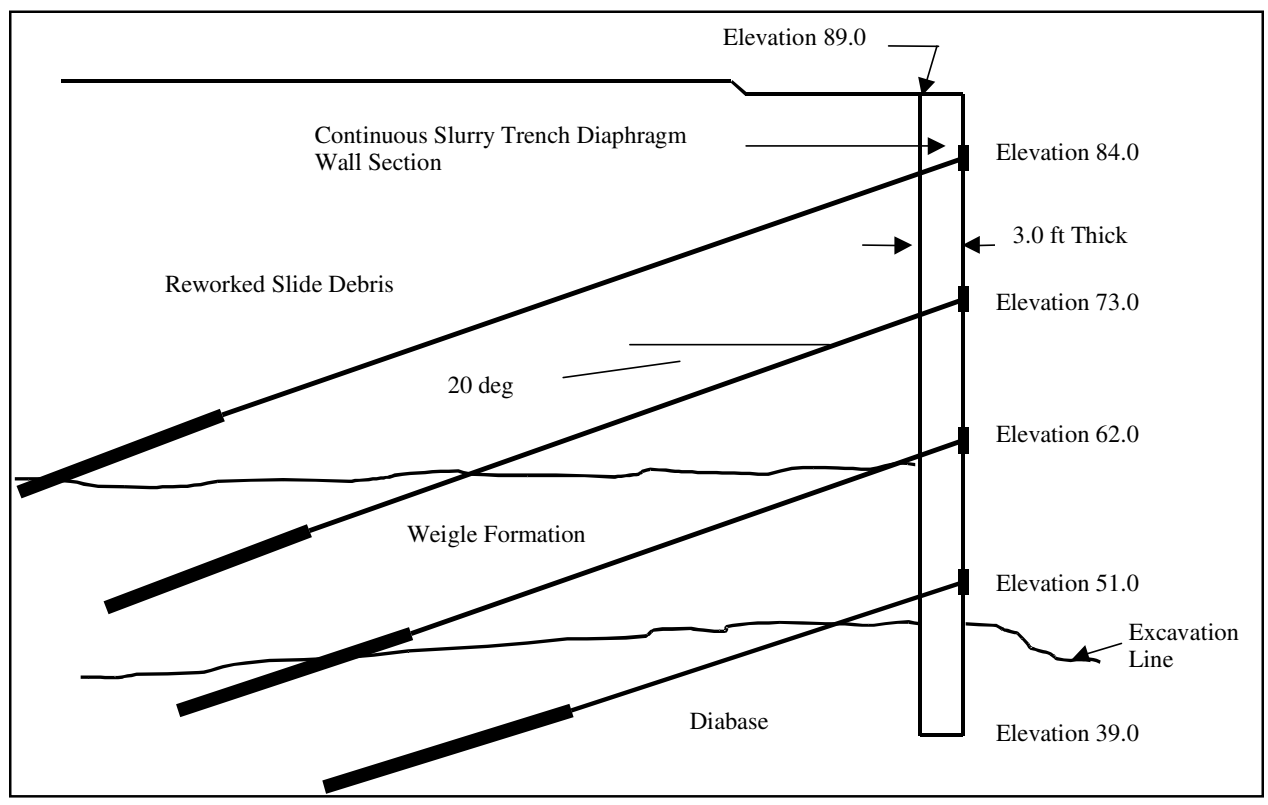

Figure 2.10. Continuous concrete slurry wall system with post-tensioned tieback anchors (Bonneville Navigation Lock temporary tieback wall)

Additional information on continuous reinforced concrete slurry wall systems can be found in Kerr and Tamaro (1990); Maurseth and Sedey (1991); Munger, Jones, and Johnson (1991); and Tamaro (1990).

\subsection{Soldier Beam Tremie Concrete System with Post-Tensioned Tieback Anchors}

A soldier beam tremie concrete system with P-T anchors is illustrated in Figure 2.5. The construction approach described above for continuous reinforced concrete slurry wall systems can also be used to construct soldier beam tremie concrete wall systems. Instead of using stop end tubes at the ends of the firststage panel sections, permanent soldier beams (usually WF beams) are used. The WF beams are fabricated with trumpet sections to accommodate the P-T tieback anchors. Reinforcement cages similar to those used for the continuous slurry wall are placed in the slurry trench between the soldier beams, and the concrete is placed as described above. In this type of wall, the reinforced concrete, rather than being the principal structural element, simply acts as lagging between soldier beams. The concrete lagging is designed as a simply supported beam spanning between soldier beams. The contribution from plate action (plate bending) is small in this type of system and therefore is not usually considered in design.

The navigation lock upstream guard wall at Bonneville Dam is a soldier beam tremie concrete system. The wall, however, served as a continuous reinforced concrete slurry wall system during construction. During excavation, temporary tieback anchors were installed in the reinforced concrete panels to 
support lateral earth pressures. Toward the end of construction, a cap beam with permanent tieback anchors was installed to support the net lateral earth pressure force (lateral earth pressure - hydrostatic pressure) that would occur after construction was complete and the site watered up. After water-up, the temporary anchors served no purpose, and the design assumed that the system performed as a typical soldier beam and concrete lagging system.

Details of the soldier beam tremie concrete system used for the navigation lock upstream guard wall at Bonneville Dam are illustrated in Figure 2.11.

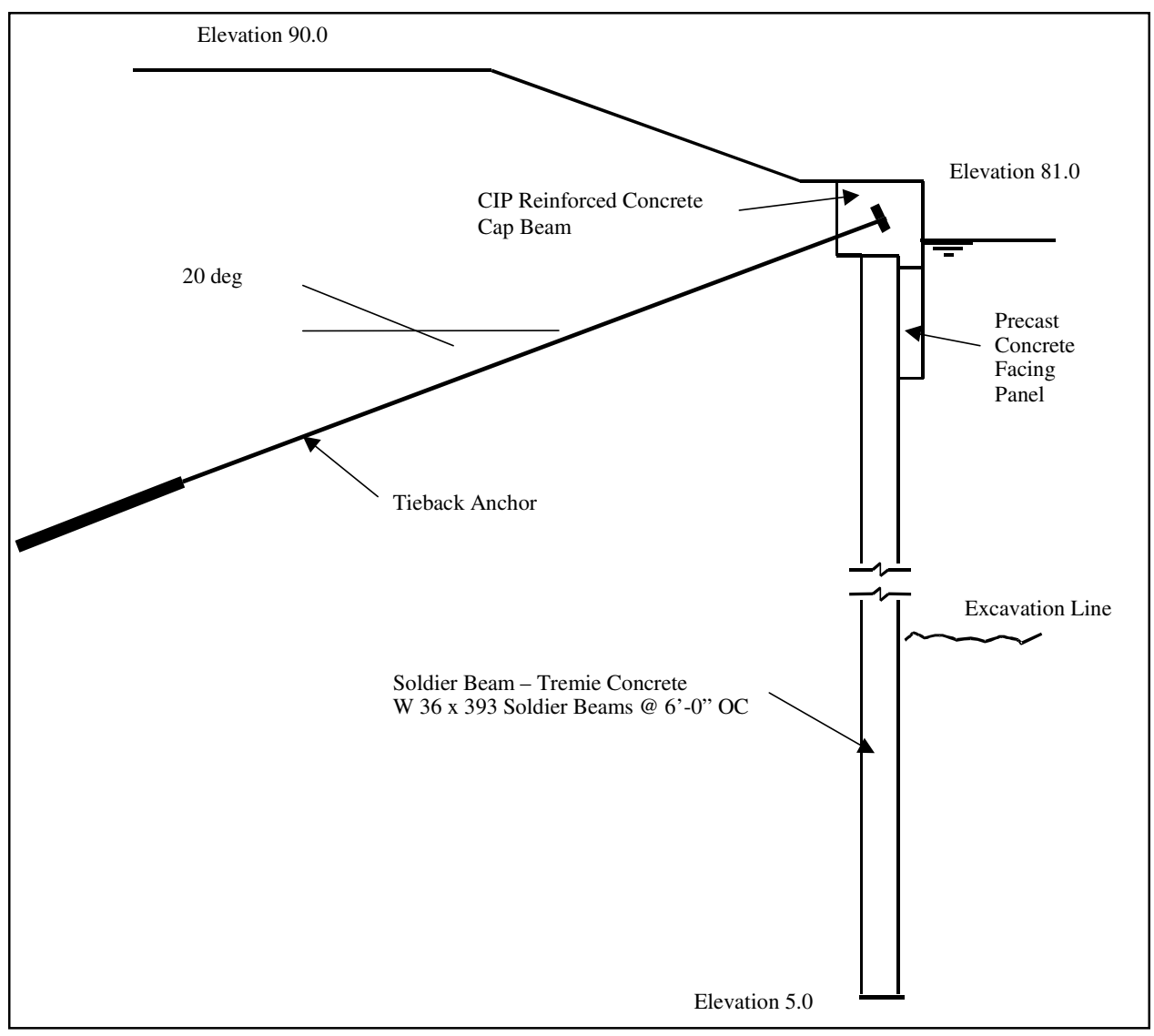

Figure 2.11. Soldier beam tremie concrete system with post-tensioned tieback anchors (Bonneville Navigation Lock upstream guard wall)

\subsection{Continuous Versus Discrete Tieback Wall Systems}

In continuous tieback wall systems, the entire wall extends below grade and is available to provide toe resistance. For such systems the resisting pressure developed at the wall toe can be based on Rankine passive pressure. A continuous tieback wall system is illustrated in Figure 2.12. Vertical sheet-pile and concrete slurry wall systems are continuous tieback wall systems. 


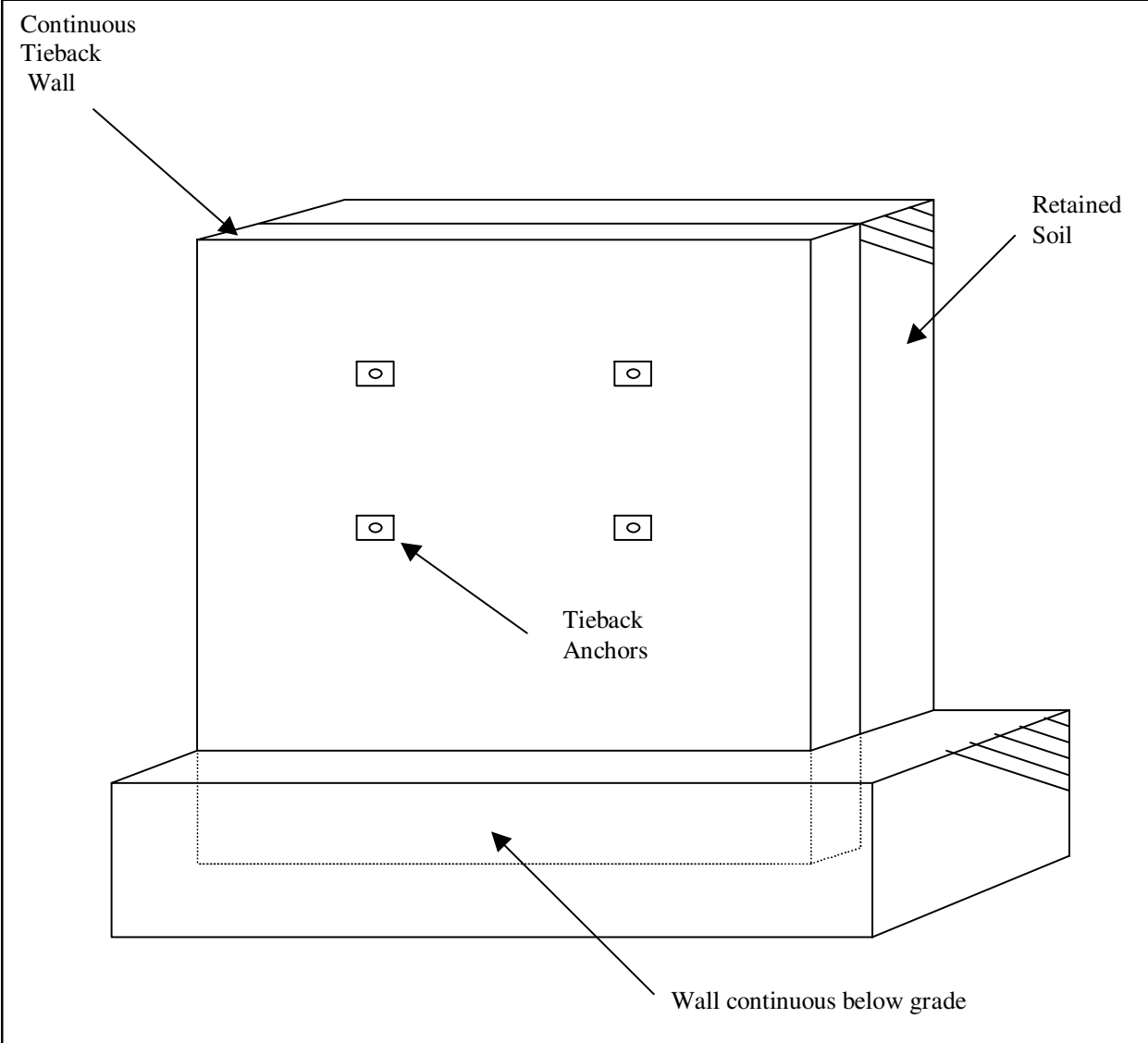

Figure 2.12. Continuous wall system

In discrete tieback wall systems (as shown in Figure 2.13), only the solider beams extend below grade. For such systems, several modes of toe failure are possible and must be investigated. These include failure of passive wedges in front of individual soldier beams, failure where individual soldier beam passive wedges overlap, and plastic flow of the soil around soldier beams. In addition, from a global perspective, at no point should the total passive resistance of a discrete system exceed that of a two-dimensional (continuous wall-type) system. The various types of failure mechanisms for discrete systems are discussed in Chapter 8. The soldier beam with wood lagging, the secant cylinder pile, and the soldier beam tremie concrete systems are all discrete tieback wall systems, although the latter two can also be constructed as a continuous system. 


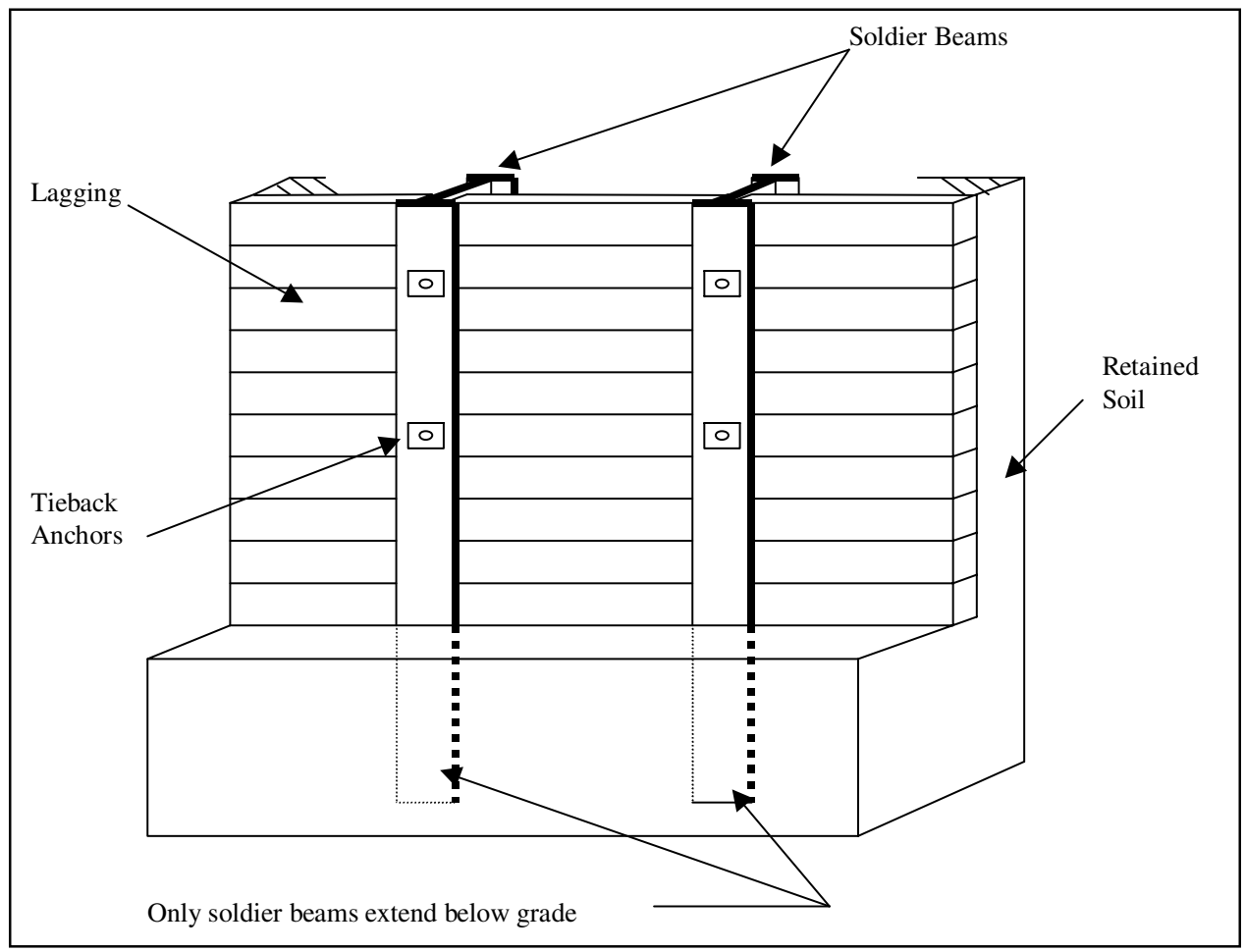

Figure 2.13. Discrete wall system 


\section{Tieback Wall Design and Design Standards}

\subsection{General}

The information in this chapter provides an overview of the tieback wall design process with respect to both temporary support of excavation walls and permanent retaining wall systems. In particular, this chapter

- Describes the general behavior of anchored wall systems.

- Describes tieback wall design and analysis procedures.

- Sets performance objectives.

- Cites applicable design codes and standards.

\subsection{Behavior of Tieback Wall Systems}

\subsubsection{General}

Excavations for deep braced cuts, for conditions where the groundwater table is below the base of the wall, generally result in a trapezoidal earth pressure distribution unlike the typical triangular distribution commonly used in the design of backfilled retaining wall systems. The trapezoidal earth pressure distribution is the result of top-down construction sequencing, causing the braced wall to rotate about the top rather than about the bottom, as is the case for a backfilled retaining wall system. The same top-down construction for flexible tieback wall systems also results in a soil pressure distribution that approximates the same trapezoidal distribution assumed for the design of braced excavation systems.

Many Corps tieback wall systems are constructed in multilayered ground conditions overlying rock. Anchored walls constructed for these types of excavation sites have been studied by Chungsik Yoo, and the results presented in the ASCE Journal of Geotechnical and Geoenvironmental Engineering (Yoo 2001). Yoo discusses the influence of wall stiffness, support conditions, and overexcavation on wall behavior with emphasis on lateral wall movement and earth pressure distribution. 


\subsubsection{Tieback wall construction sequencing}

The construction sequencing with respect to a flexible tieback wall with two anchors is illustrated in Figures 3.1-3.5. The tieback wall system is shown in Figure 3.1. In this case, the tieback wall system is a conventional soldier beam system with timber lagging. The water table is assumed to be below the base of the wall.

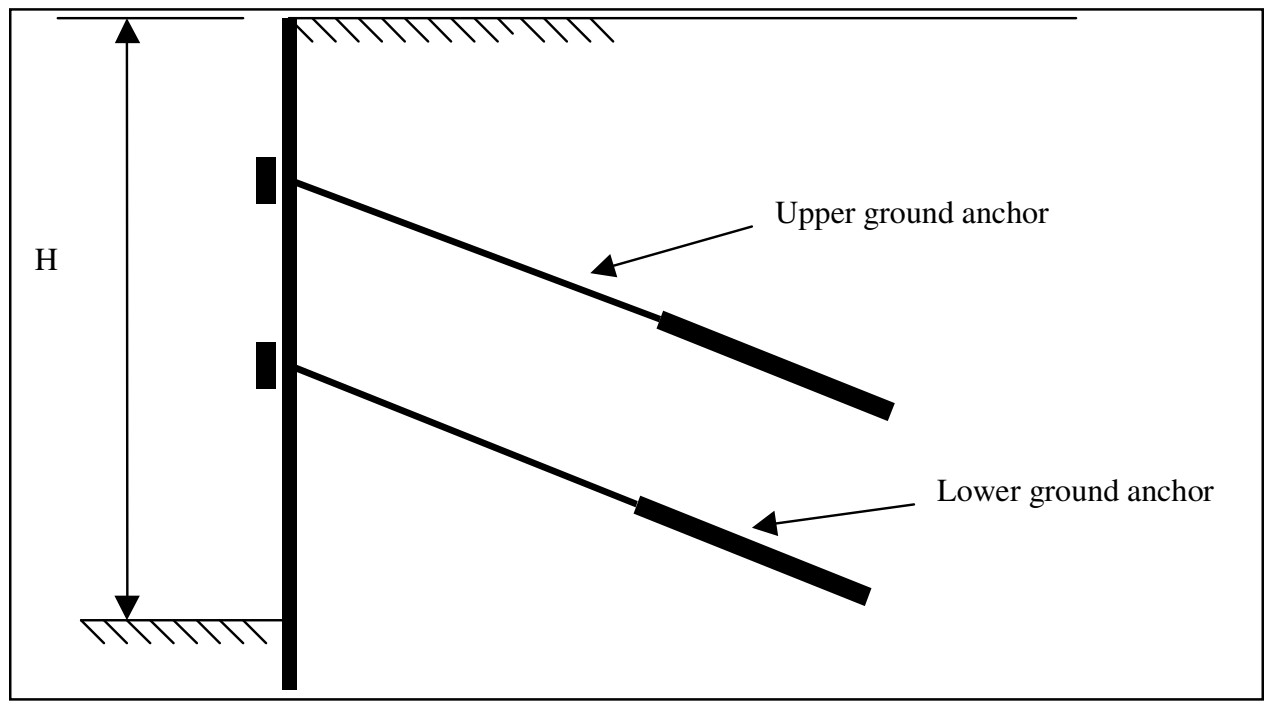

Figure 3.1. Tieback wall system

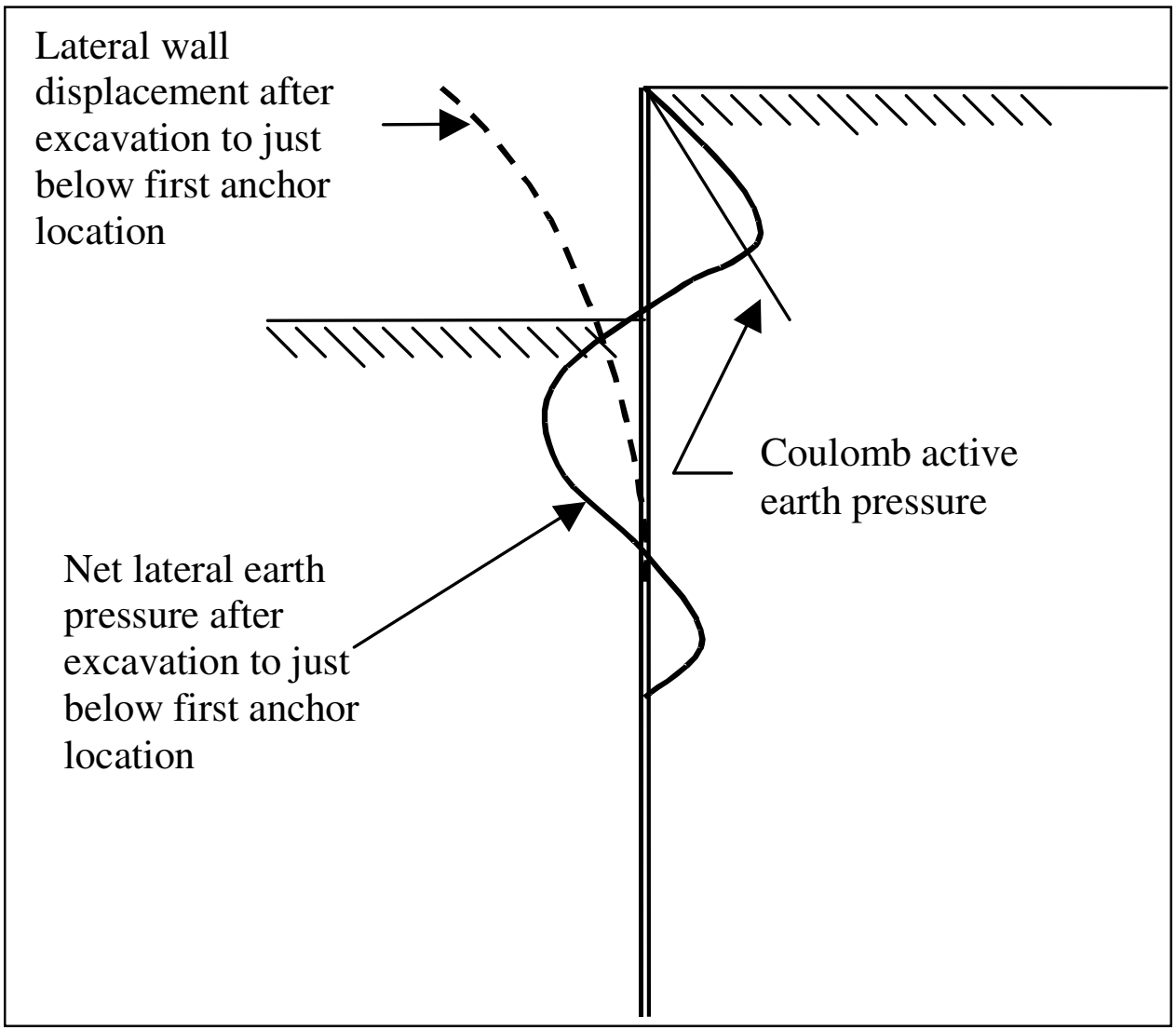

Figure 3.2. First-stage excavation-lateral wall movements and earth pressures 


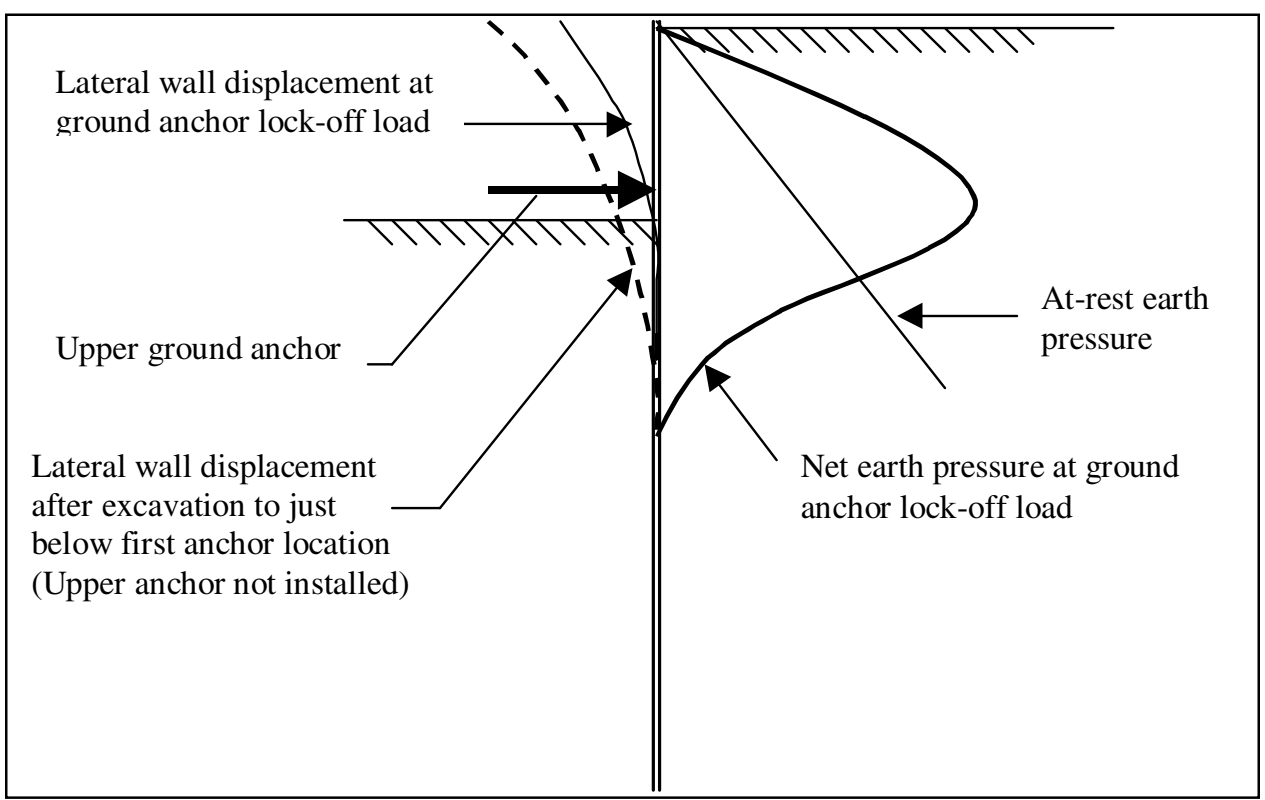

Figure 3.3. Upper tieback installation-lateral wall movements and earth pressures (after Sabatini, Pass, and Bachus 1999)

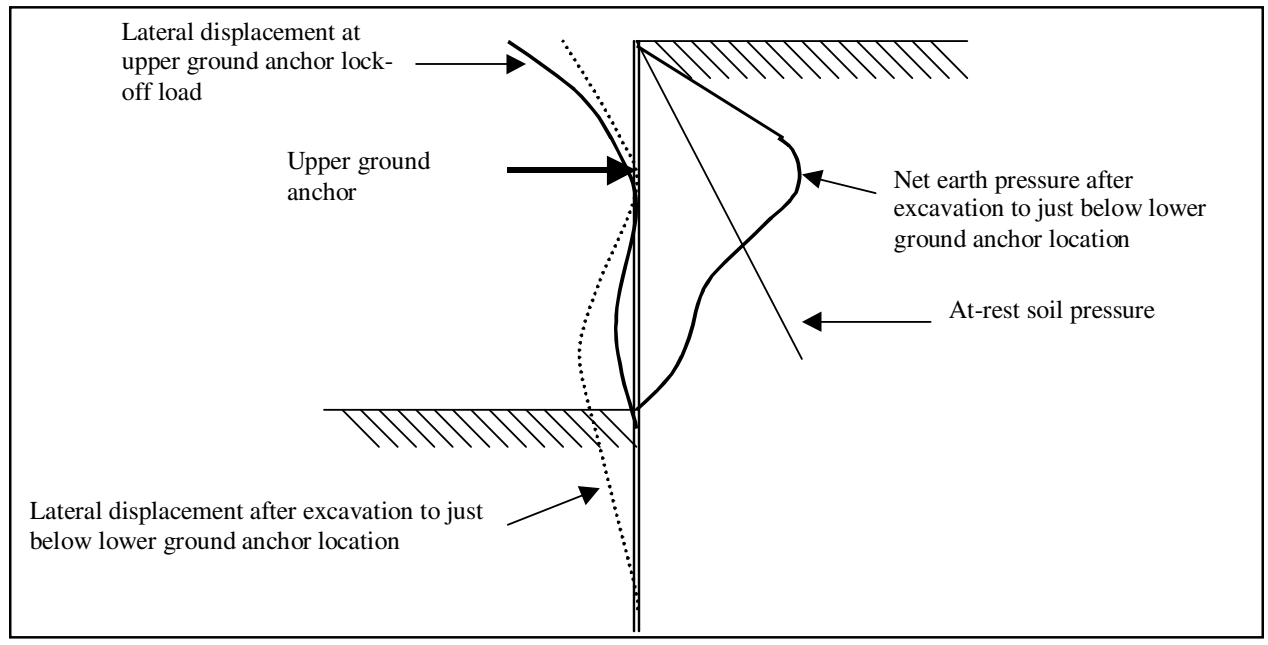

Figure 3.4. Second stage excavation-lateral wall movements and earth pressures (after Sabatini, Pass, and Bachus 1999) 


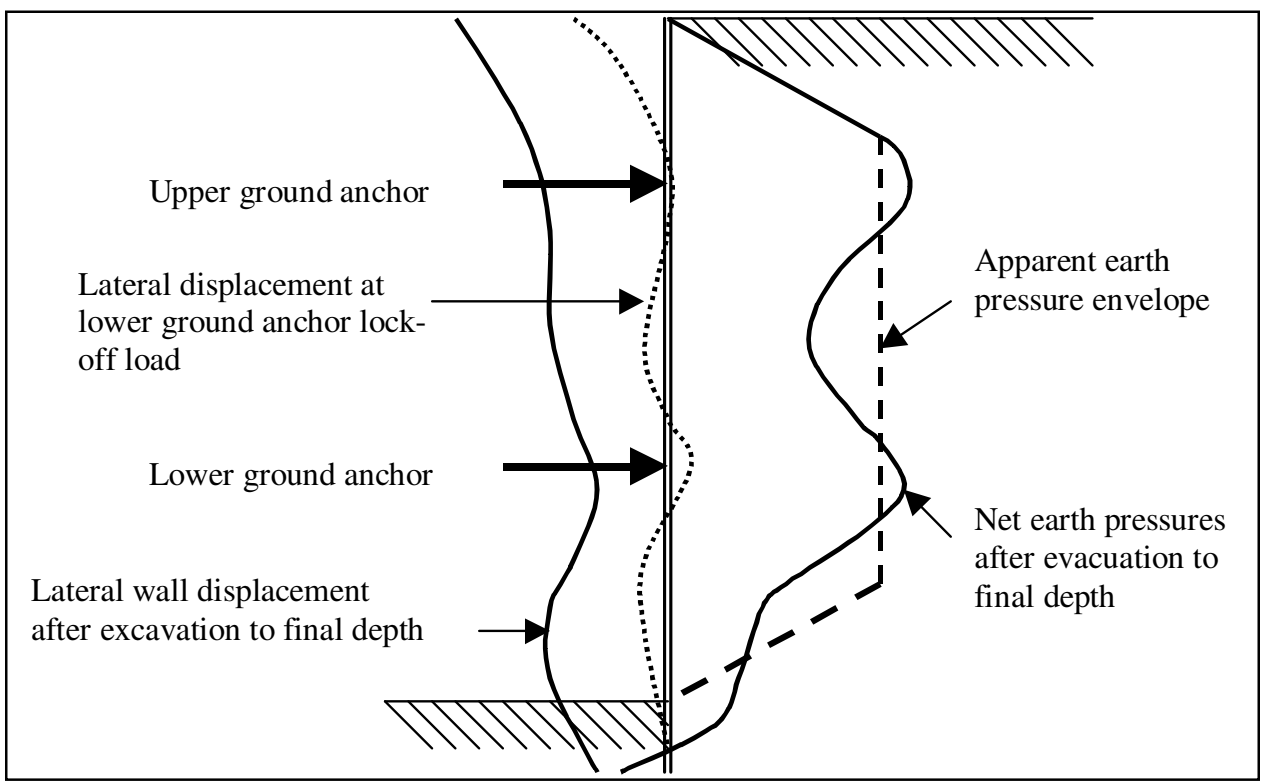

Figure 3.5. Lower tieback installation-final-stage excavation-lateral wall movements and earth pressures (after Sabatini, Pass, and Bachus 1999)

In the first stage of construction, the soldier beams are drilled or driven to the required depth. First-stage excavation is closely followed by the installation of timber lagging. First-stage excavation continues to a depth that will permit installation of the upper tieback anchor. At this stage the pressures on the wall are active pressures, with the wall acting as a cantilever. First-stage earth pressures and lateral wall movements are illustrated in Figure 3.2.

The upper tieback anchor is then installed and tensioned as required to obtain a final design anchor force accounting for all short- and long-term losses. During this stage of construction the anchor force will result in earth pressures that exceed at-rest pressures, and can approach passive pressure conditions within regions of the soil immediately adjacent to the anchor. It should be noted that high prestress loads in the upper anchor could lead to passive earth failure if the location of the upper anchor is too shallow. Earth pressures and lateral wall movements after lock-off of the upper tieback anchor are as illustrated in Figure 3.3.

Following installation of the upper anchor, excavation takes place to a depth that will permit installation of the lower tieback anchor. At this stage, earth pressures and lateral wall movements are as illustrated in Figure 3.4.

The lower ground anchor is then installed and excavation continues until the final excavation depth $(H)$ is reached. The earth pressures and lateral wall movements at the end of construction are shown in Figure 3.5. As illustrated, the final earth pressures for the flexible tieback wall system approximate a trapezoidal distribution. 


\subsubsection{General behavior and design methodologies-focus wall systems}

3.2.3.1 General. Five tieback wall systems were selected for specific consideration in this report. These represent tieback wall systems used at various Corps projects. Procedures used in their design suggest that the lateral earth loadings and pressure distributions used for design were based on assumptions made with respect to specific, yet unspecified, load-displacement behavior. Earth pressure loads and load pressure distributions selected for the design of flexible sheet pile, and for the soldier beam with wood lagging systems, often differ from those used for the design of more rigid secant pile, slurry wall, and soldier beamtremie concrete systems. Differences with respect to the design of flexible and stiff tieback wall systems are described in the following sections. It should be realized that there are many variations with respect to the design approaches described in this report.

3.2.3.2 Flexible tieback wall systems. In general, sheet-pile walls and soldier beam and lagging walls are considered flexible wall systems and often exhibit behavior similar to that described in Section 3.2.2. Design usually proceeds on the assumption that the use of apparent pressures in a final stage analysis will produce a wall capable of resisting all earth pressure experience during and after construction. Apparent pressure diagrams, similar to Figure 3.6, are used in the analysis. The total load represented by the apparent pressure diagram is often based on active earth pressures with a factor of safety approximately equal to 1.3 applied to the shear strength of the soil. The tieback locations are generally considered to be rigid supports.

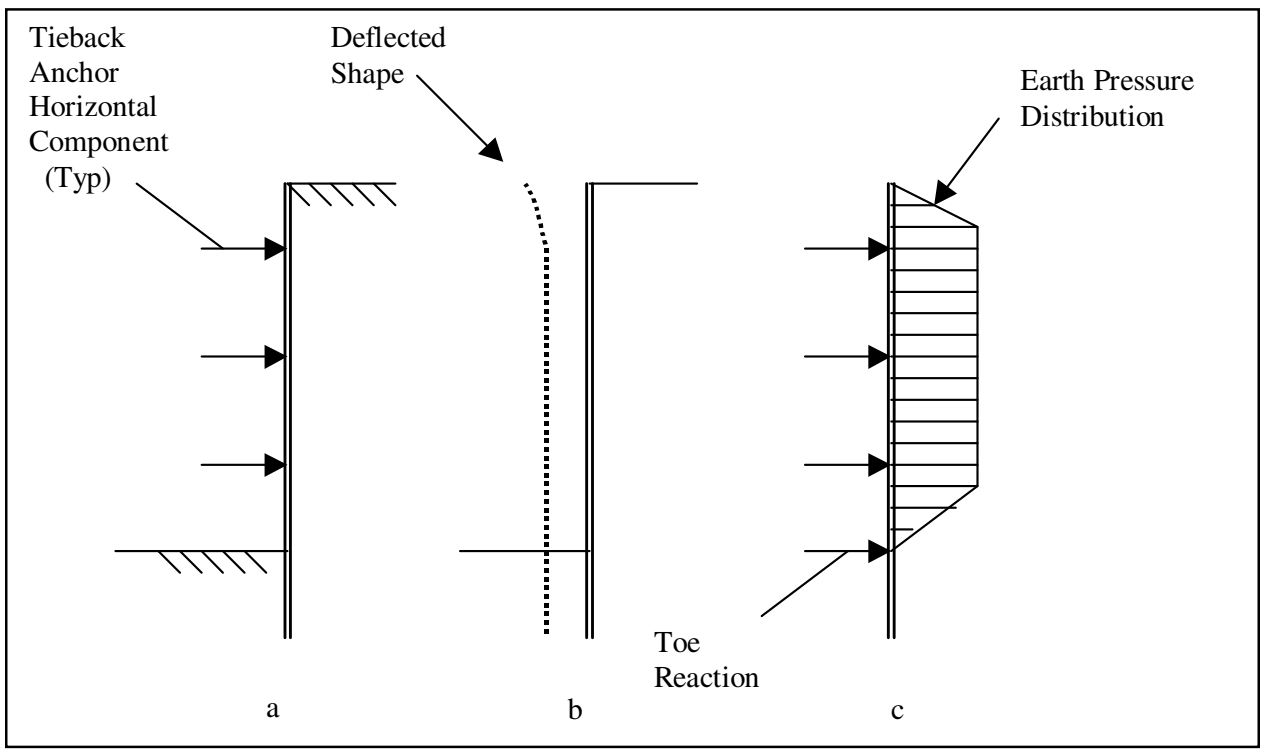

Figure 3.6. Trapezoidal (apparent pressure) earth pressure distribution

Simple analytical models composed of an equivalent beam on rigid supports are used for the analysis. This type of analysis (for the purposes of this report) is designated as a RIGID analysis. The RIGID analysis is described in general 
terms below, with details provided in Chapter 5. Ground anchor loads are determined based on the earth pressure tributary to each anchor location. Simple equations are used to conservatively estimate sheet pile and soldier beam bending moments. Flexible walls with multiple tiebacks are likely to experience nearly uniform translation, and the use of a trapezoidal pressure distribution as represented by apparent pressure diagrams will, in most circumstances, provide a reasonable design.

3.2.3.3 Stiff tieback wall systems. Secant pile, slurry wall, and soldier beam-tremie concrete walls are relatively thick and therefore more rigid than the systems described in the preceding section. These walls are often keyed into rock, thereby restricting toe movement. Since displacements are restricted due to wall stiffness and toe restraint, the magnitude of the soil pressures is often greater, and the pressure distribution is different from that of the more flexible systems described above. Triangular soil pressure distribution is often assumed for the design (see Figure 3.7).

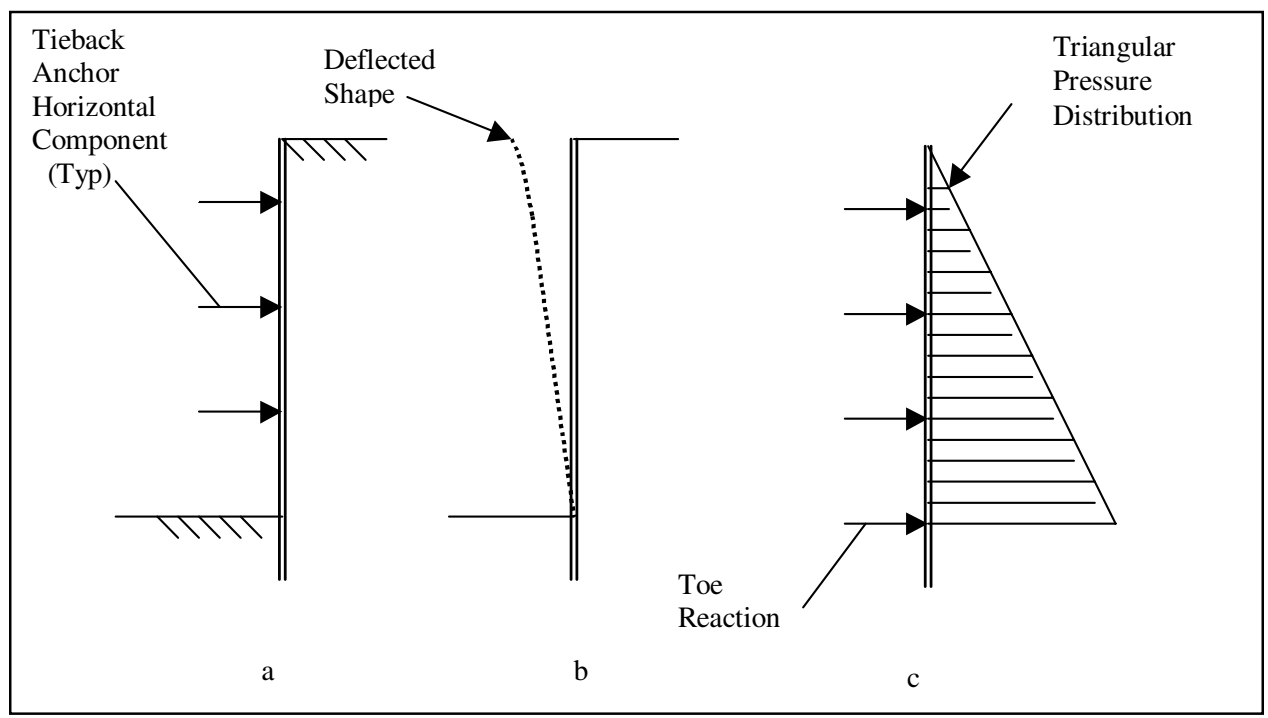

Figure 3.7. Triangular earth pressure distribution

In some cases a more conservative composite trapezoidal/triangular soil pressure distribution is used (see Figure 3.8). This approach was used for the preliminary design of the Bonneville Navigation Lock temporary tieback wall. 


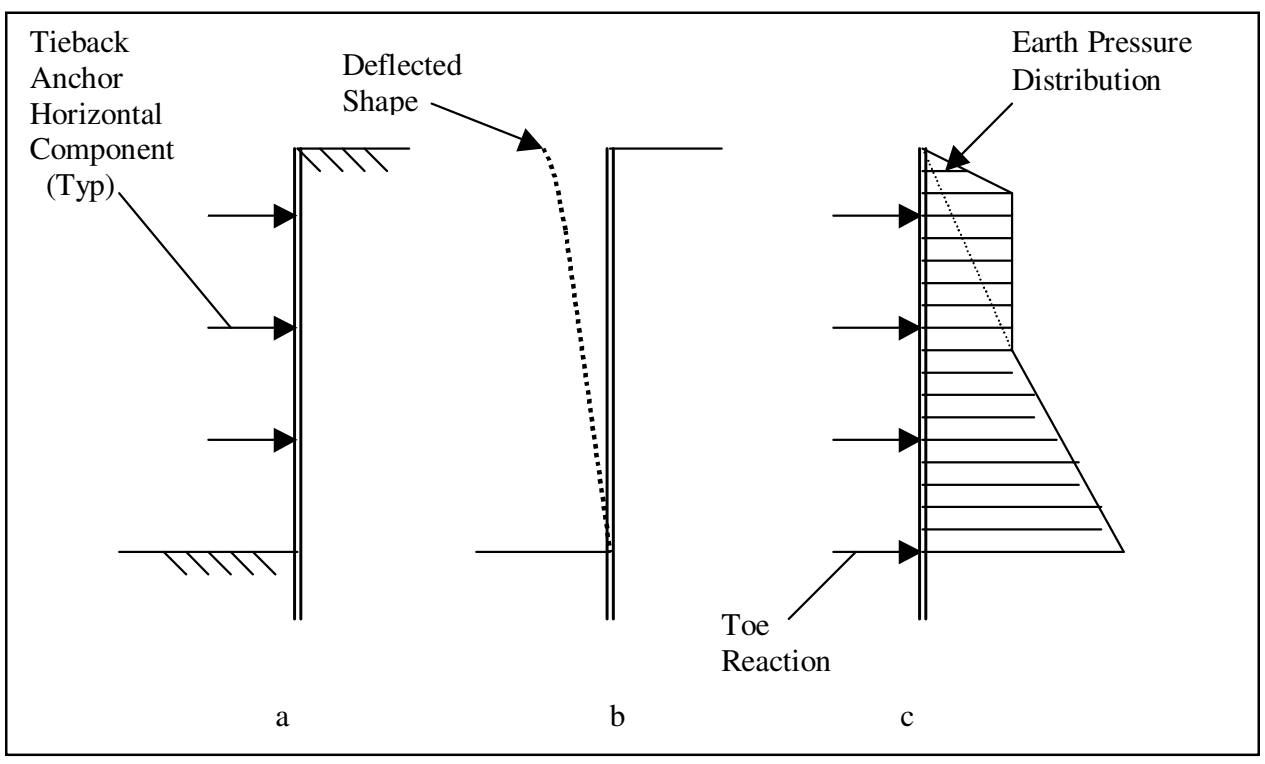

Figure 3.8. Composite earth pressure distribution

The total earth pressure load used in the design of stiff tieback wall systems is usually based on nonyielding (at-rest), or something approaching nonyielding, soil conditions. For the analysis, some designers will use at-rest pressures and others will use an intermediate value somewhere between active and at-rest. The choice depends on the level of prestressing provided by the anchors, with higher prestress levels used where displacement control is a performance objective. In general, the simpler methods of analysis assume rigid support conditions, although methods that account for wall movements at supports (anchor locations) are becoming an important part of the numerical procedure (Kerr and Tamaro 1990).

Staged analysis is often required in the design of the stiffer secant pile, slurry wall, and soldier beam-tremie concrete wall systems. In a staged analysis, tieback loads, bending moments, shears, and possibly deflections are computed for each stage of excavation. Anchor forces and wall bending moments are determined by indeterminate structural analysis. Winkler spring-type analyses can also be used to evaluate staging effects. A Winkler spring analysis was used in the design of the Monongahela River Locks and Dam 2, secant pile wall. The Winkler analysis is described in general terms below with specific details provided in Chapter 6. Although not commonly used, nonlinear finite element-soil structure interaction (NLFEM) analyses can provide valuable insight as to the actual behavior of the soil-structure system. This type of analysis may be necessary when ground displacement control is critical to project performance. Information on NLFEM analysis can be found in Chapter 7 . 


\subsection{Tieback Wall Design and Analysis Procedures}

\subsubsection{General}

Four methods are used to design and evaluate tieback wall systems:

- The beam on rigid supports (RIGID) method.

- The beam on elastic supports (WINKLER) method.

- The linear-elastic finite element (LEFEM) method.

- The nonlinear finite element-soil structure interaction (NLFEM) method.

In general practice, the use of soil pressure envelopes as loadings for a beam on rigid support analysis provides an expedient method for the initial layout, and sometimes the final design, of tieback wall systems. However, the soil pressure envelopes, or apparent earth pressure diagrams developed by Peck (1969) and as presented in Peck, Hanson, and Thornburn (1974), were not intended to represent the real distribution of earth pressure, but instead constituted hypothetical pressures. These hypothetical pressures were a basis from which there could be calculated strut loads that might be approached but would not be exceeded in the actual cut.

The design procedures used in Federal Highway Administration publications (Sabatini, Pass, and Bachus 1999; Weatherby 1998) employ apparent pressure diagrams that are modifications of the apparent earth pressure diagrams developed by Peck. These modified apparent pressure diagrams are used to provide reasonable estimates of ground anchor loads and to provide conservative estimates of wall bending moments between anchors for flexible walls constructed in competent soils. In addition, FHWA procedures allow the total load on the wall as determined from limiting equilibrium analysis to be used as a basis for developing a trapezoidal pressure distribution representing apparent earth pressures. Long, Weatherby, and Cording (1998) demonstrated that the total loads from Terzaghi and Peck's (1967) sand and soft-to-medium clay are equal to the total lateral loads using limiting equilibrium analysis with a factor of safety of about 1.3 on shear strength.

The determination of the ground anchor force required to support a cut using limiting equilibrium analysis, or slope stability computations, is consistent with an extension of the original work of Terzaghi and Peck (1967). The use of limiting equilibrium procedures has several advantages in that it is a more general formulation of the boundary condition problem, and therefore, can accommodate changes in the wall geometry, strength properties, and groundwater conditions in a more fundamental way. However, the use of limiting equilibrium or slope stability computations requires that a consistent definition for a factor of safety be used (Long, Weatherby, and Cording 1998). The factor of safety can be applied as a strength mobilization factor to the soil shear strength ( $F S_{\text {Strength }}$ method) as described in Engineer Manual (EM) 1110-2-2502 (Headquarters, Department of the Army (HQDA) 1989), which is the preferred method for limiting equilibrium analysis. Or, it can be applied to the soil load 
( $F S_{\text {Load }}$ method). Both methods are used in practice, and both give similar but somewhat different answers with respect to the total load required to support the tieback wall cut. This issue is discussed further in Long, Weatherby, and Cording (1998). With respect to the state-of-the-practice information presented in this report it should be assumed that, where total lateral earth pressure loads are estimated based on limit equilibrium analysis, the factor of safety is applied to the shear strength of the soil.

The total earth load determined by limiting equilibrium methods can be converted to a FHWA apparent soil pressure diagram for use in the design of tieback walls (Weatherby 1998). Also, by increasing the factor of safety, conditions approaching at-rest pressures can be simulated. This allows the engineer to develop apparent pressure diagrams for use in the design of stiff wall systems, and for use in the design of wall systems requiring stringent displacement control. Some cautions should be observed, however, when using apparent pressure diagrams as actual pressure diagrams. As stated by Liao and Neff (1990):

Trapezoidal earth pressure distributions proposed by Peck (1969) for dense soils and stiff fissured clays can theoretically reflect the shapes of the actual (rather than apparent) pressure distributions behind a wall. However, it is more likely that nontriangular distributions will occur in overconsolidated dense sands and stiff to hard clays. For loose sands and soft clays, the actual pressure distributions will tend to be more triangular in shape.

In practice, the specified apparent earth pressure diagrams are sometimes not distinguished from actual earth pressures, and structural designers often treat them equivalently. Given the present availability of microcomputers and structural analysis programs, and the ease of solving indeterminate structural problems, the original intended application of Peck's apparent earth pressure diagrams have fallen into disuse. Instead, structural designers often assume that the earth pressures are the actual earth pressures, using them as input to analysis, i.e. they avoid the use of the tributary area method for calculating strut loads. This procedure is clearly improper, considering the derivation of apparent earth pressures, although it is not clear whether the procedure leads to overly conservative or unsafe designs. It is the writer's opinion that, in order to rectify this situation, geotechnical engineers should indicate clearly when they are specifying actual earth pressures versus apparent earth pressures, and indicate the applicable method for calculating strut loads. 


\subsubsection{RIGID analysis procedure}

The procedures developed by FHWA (Sabatini, Pass, and Bachus 1999) are commonly used in the design of flexible vertical sheet-pile and soldier beam systems. Various aspects related to the FHWA approach are discussed in later chapters of the report. The designer must keep in mind that earth pressures are a function of wall displacements and the FHWA apparent pressure diagrams represent an envelope that can be used to develop an adequate anchor system for flexible sheet-pile systems and soldier beam systems.

The FHWA diagrams are used as part of an equivalent beam on rigid supports (RIGID) analysis method. With the RIGID analysis procedure, it is assumed that the principal structural elements (e.g. soldier beam, sheet pile, secant pile) span vertically between ground anchor supports that are assumed to be rigid (i.e., zero lateral displacement of the structural element at ground anchor locations). Although this method provides reasonable results in most cases, it must be remembered the assumptions made with respect to boundary conditions at ground anchor supports are fictitious. In the actual case, the wall does displace at anchor locations, and in the more sophisticated analyses these displacements are considered in the analysis. In most cases, however, rigid support boundary conditions provide reasonable results with respect to bending moments and shears in soldier beams, sheetpiling, secant piles, etc. It also should be remembered that the apparent pressures represent a load envelope and not the actual loads that might exist on the wall at any time. The apparent pressure diagrams also assume that

- The excavation is very deep (greater than $6 \mathrm{~m}(20 \mathrm{ft})$ ) and relatively wide, and wall movements are sufficient to mobilize essentially the full value of soil strength.

- Groundwater is below the base of the cut for sands and for clays. Its position is not considered to be influential.

- Soil masses are assumed homogeneous.

- The diagrams are primarily intended for calculating anchor loads.

- Recommended pressures are conservative envelope values intended to account for widely varying observed field behavior and ranges of theoretically predicted behavior.

- Loading diagrams apply only to the exposed portion of the walls and not to sections embedded in the ground.

Conditions other than those described above are not uncommon. Although the use of apparent pressure diagrams to design tieback structural support systems has wide application, the process must often be modified. The FHWA (Sabatini, Pass and Bachus 1999) has presented techniques that can be used in an apparent earth pressure analysis in order to 
- Meet project performance objectives required for permanent construction.

- Meet stringent displacement control requirements.

- Account for long-term as well as short-term loadings.

- Account for rigid wall behavior.

- Account for hydrostatic and unique surcharge loadings.

- Account for stratified and irregularly configured soil masses.

- Achieve higher margins of safety.

As stated previously, in the case of the stiffer wall systems the actual pressures might tend to be more triangular in shape. In such cases a RIGID analysis using both trapezoidal and triangular distribution of total load should be considered.

\subsubsection{WINKLER analysis}

Where assessment of actual loads on the tieback wall is required for all stages of excavation, a staged construction analysis using beam on elastic foundation (Winkler) analysis techniques may be warranted. The Winkler type analysis should also be considered when

- The wall is influenced by loadings from nearby structures.

- Large surcharge loadings need to be resisted by the wall.

- Pre-existing instabilities or planes of weakness exist in the retained soil.

- Wall movements differing from those assumed by the RIGID analysis (i.e. stiff wall system, wall embedded in rock) are likely.

Since actual wall loadings are approximated by a Winkler type analysis, the moments and shears on the wall can be determined by indeterminate structural analysis techniques rather than by the tributary area method. It must be remembered, however, that the displacements resulting from the Winkler indeterminate structural analysis are sensitive to the stiffness selected for the soil springs and, therefore, the analysis will likely not produce displacements that are representative of the actual wall displacements.

Beam on elastic foundation analyses are able to match experimental results better than RIGID analysis solutions since they can take into account soil loaddeformation characteristics, wall stiffness, anchor elasticity, and plastic (nonrecoverable) movements in the soil. When they are used in a staged construction analysis, they usually provide moments and shears that are realistic in both magnitude and distribution for each construction stage as well as for final excavation. Although the displacements from the WINKLER analyses may not accurately reflect actual wall displacement, they permit engineers to qualitatively evaluate the effects anchor spacing and prestressing have on wall displacements (i.e., reduced or increased movements) before undertaking more complicated nonlinear finite element method analyses. 


\subsubsection{Linear elastic and nonlinear finite element method analyses}

Linear elastic finite element method analyses are sometimes used to model diaphragm (continuous) wall systems in combination with linear Winkler soil springs. In the linear elastic model, the soil and water on the unexcavated side of the wall are modeled as loads, and the soil on the excavation side is modeled as linear elastic (Winkler) springs preloaded to at-rest pressure conditions. The forces in the springs are monitored during a staged excavation analysis to determine if they exceed passive pressure resistance. If this occurs, a passive pressure force replaces the spring. In more sophisticated LEFEM analyses, bilinear excavation side springs are used, with the plastic region of the bilinear curve representing the passive limit state of the soil. On the active (unexcavated) side, the pressures on the wall are applied as distributed loads. The soil active pressure is assumed to be unchanged throughout the entire sequence of excavation. The LEFEM analysis procedure is described in ASCE/SEI (2000). The diaphragm wall LEFEM is illustrated in Figure 3.9.

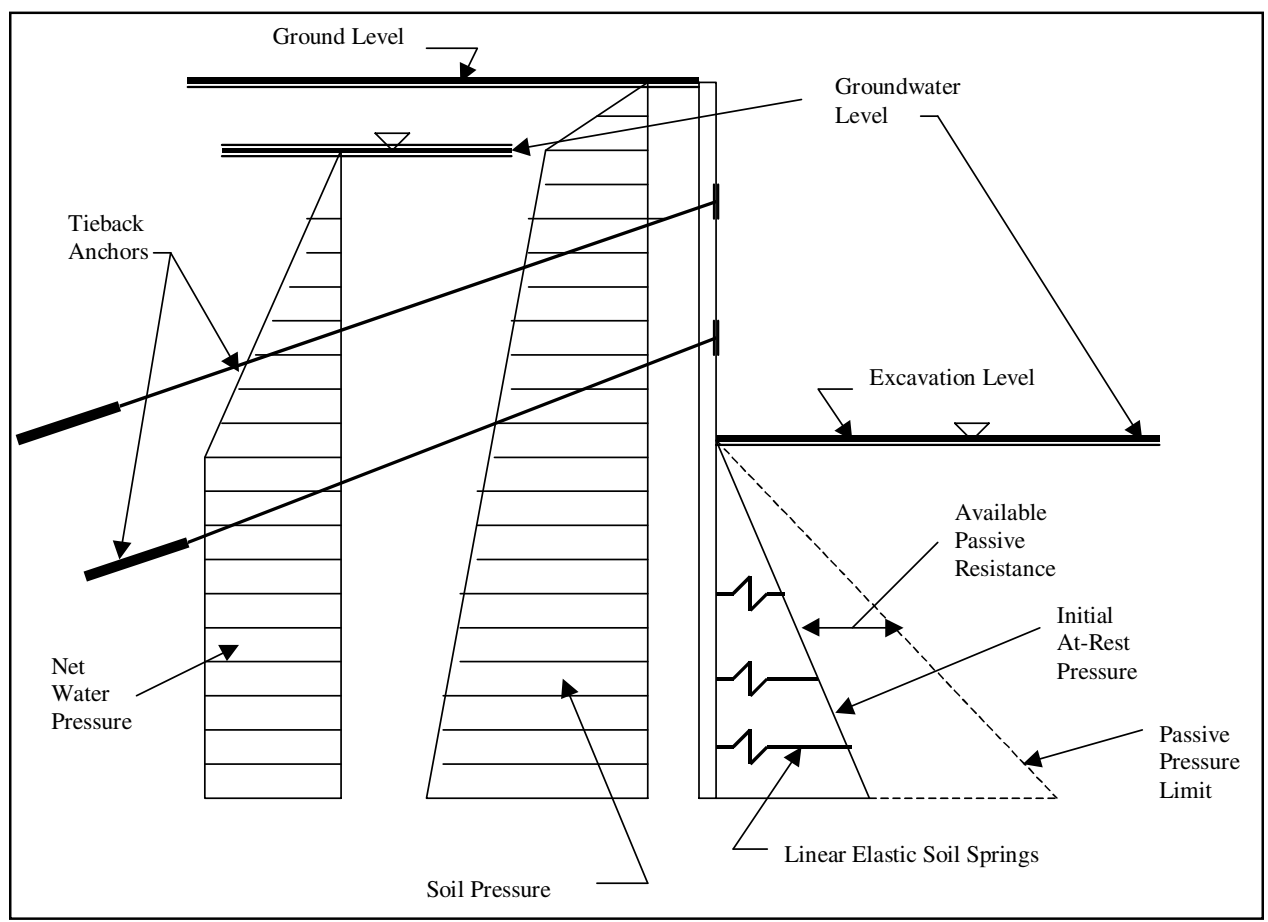

Figure 3.9. Linear elastic finite element model (LEFEM) of diaphragm wall in combination with linear Winkler soil springs

When displacements are important with respect to project performance objectives, a nonlinear finite element-soil structure interaction analysis should be performed. In an NLFEM analysis, soil material nonlinearities are considered. The analysis of diaphragm walls using NLFEM analysis procedures is described in Mosher and Knowles (1990) and in ASCE/SEI (2000).

Displacements are often of interest when displacement control is required to prevent damage to structures and utilities adjacent to the excavation. To keep displacements within acceptable limits, it may be necessary to increase the level of prestressing beyond that required for basic strength performance. An increase 
in tieback prestressing is often accompanied by a reduction in tieback spacing. As tieback prestress is increased, wall lateral movements and ground surface settlements decrease. This is illustrated in Figure 3.10.

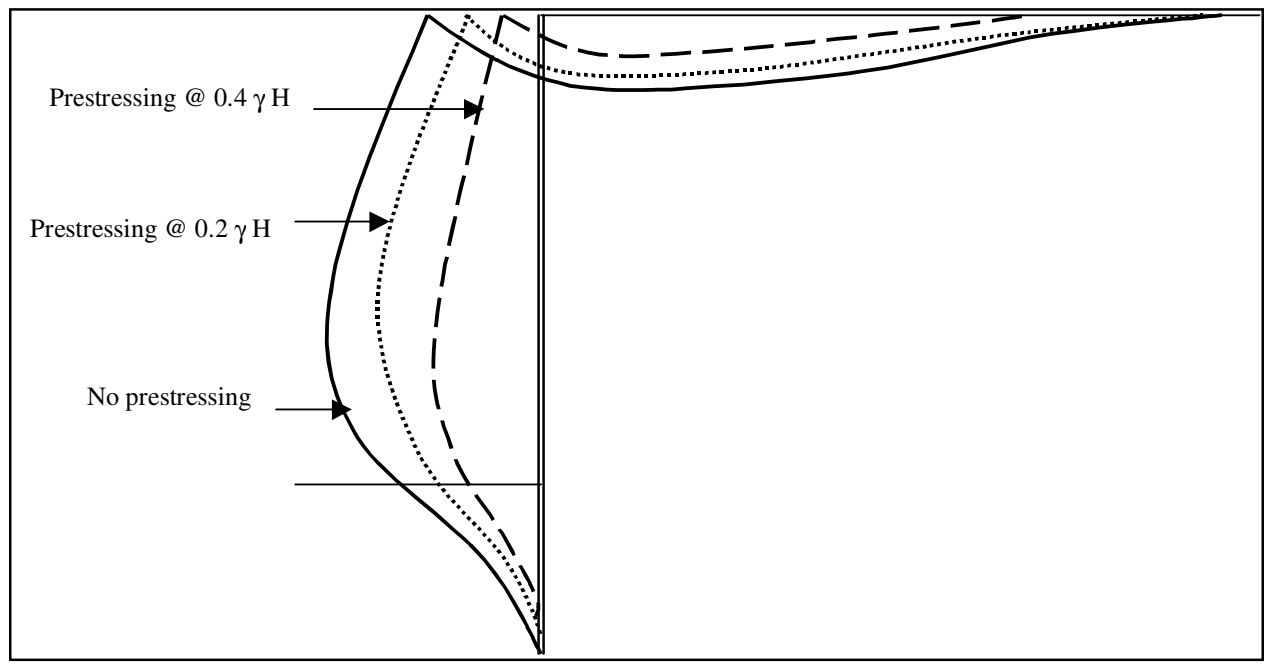

Figure 3.10. Anchor prestress versus lateral wall movement and ground settlement

Associated with an increased level of prestress is an increase in soil pressures. The higher soil pressures increase demands on the structural components of the tieback wall system. The relationship between soil pressure and tieback prestress is illustrated in Figure 3.11.

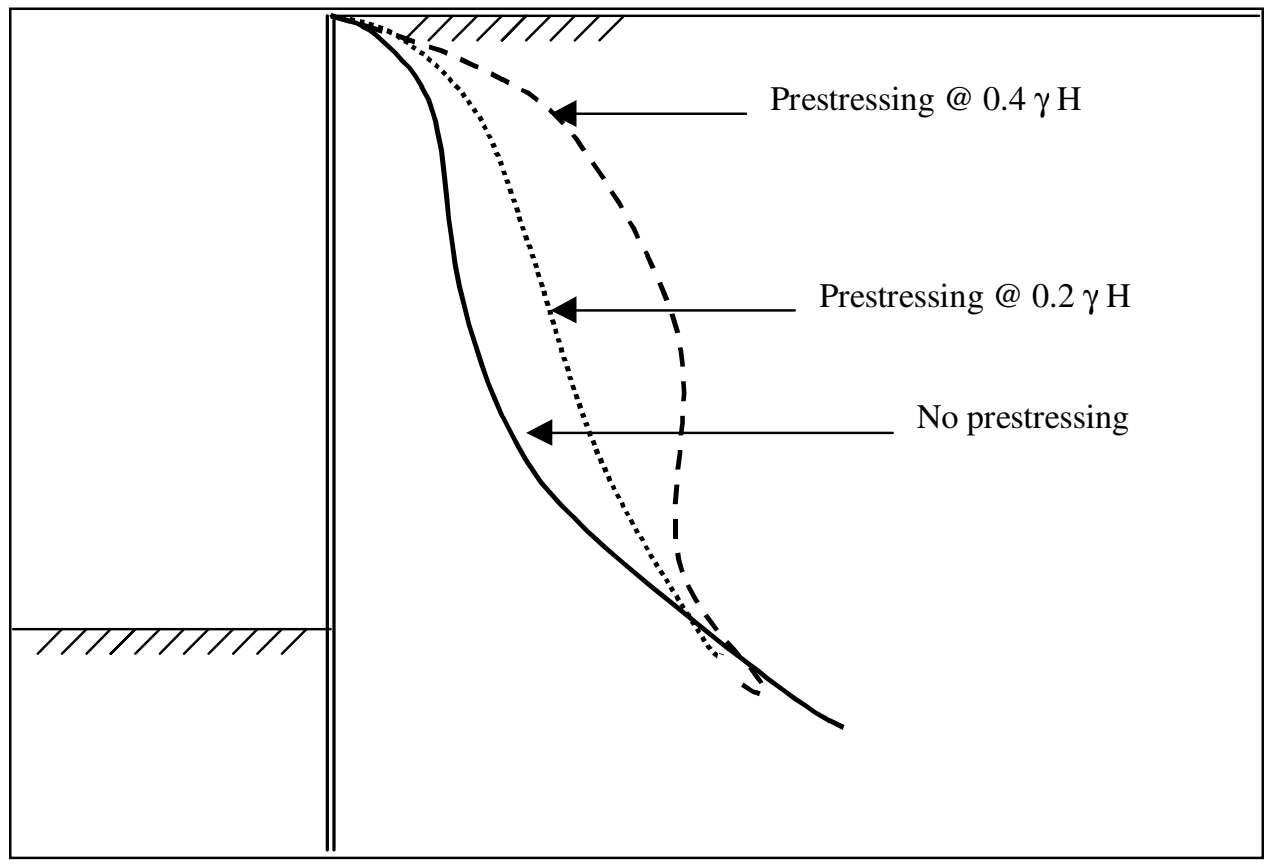

Figure 3.11. Anchor prestress versus soil pressure 
General-purpose NLFEM programs for two-dimensional plane strain analyses of soil-structure interaction problems are available to assess displacement demands on tieback wall systems. These programs can calculate displacements and stresses due to incremental construction and/or load application and are capable of modeling nonlinear stress-strain material behavior. An accurate representation of the nonlinear stress/strain behavior of the soil is essential if this type of analysis is to provide meaningful results. The simulation of incremental construction may include embankment construction or backfilling, excavation, installation of a strut or tieback anchor, excavation support system, removal of the same system, and the placement of concrete or other construction materials. The incremental loading simulation may consist of the application of concentrated loads, boundary pressures, or loads due to temperature changes in nonsoil materials.

\subsubsection{Additional analyses}

The use of standard apparent pressure diagrams in an equivalent beam on rigid support (RIGID) analysis for the design of structural support systems may need to be supplemented with the other analytical procedures described above. In addition, other analysis techniques are needed to

- Evaluate the axial and lateral load capacity of the embedded portion of the wall.

- Ensure overall stability of the anchored ground mass.

- Provide suitable corrosion protection for embedded metals.

- Determine lateral and vertical movements of the anchored soil mass.

- Evaluate the tieback wall system for a loss of anchor condition.

\subsection{Phased Approach}

Once a tieback wall system has been chosen for use at a particular project, a planned phased approach to the design and construction of the tieback wall system begins.

The steps in a phased approach for the design and construction of a tieback wall system include the following:

Step 1. Determination of project performance requirements.

Step 2. Soils explorations and testing for site, soil, and foundation characteristics as needed to

- Establish suitable construction methods.

- Determine short- and long-term wall loadings. 
- Determine foundation lateral and bearing capacities for use in the design of wall embedments.

- Establish soil and foundation strength and stiffness for soilstructure interaction analyses.

- Determine groundwater elevations and drainage requirements.

- Determine the corrosive potential of soils and select appropriate corrosion protective systems.

- Select anchor dead-end locations and determine anchor development lengths.

- Determine potential failure planes for stability analyses.

Step 3. Selection of loads and load combinations to be used in the design, including

- Determination of apparent earth pressures and/or other earth pressure loadings that best represent wall displacement and anchor prestress conditions.

- Determination of loadings to represent surcharge conditions.

- Determination of hydrostatic loads and seepage forces.

Step 4. Design of the tieback wall structural support system to established design codes and practices.

Step 5. Postdesign analysis of the tieback wall system using appropriate soil strength analyses, bearing capacity analyses, slope stability analyses, soil-structure interaction analyses (Winkler spring, LEFEM, and/or NLFEM type), and corrosion analyses when necessary to ensure that

- The design reflects actual soil-structure interaction behavior.

- Wall embedments are adequate.

- The ground mass is stable for all potential failure planes.

- The wall meets all strength, serviceability, and displacement control performance objectives.

Step 6. Preparation of contract specifications to ensure that the constructed wall meets short- and long-term performance objectives.

Step 7. Load testing and monitoring of tieback wall system performance. 


\subsection{Performance Objectives}

\subsubsection{General}

Each tieback wall project will have one or more performance objectives. As a minimum, the project must be designed to provide suitable protection against failure (Collapse Prevention Performance Objective). This may be the only objective with respect to the design of temporary support of excavation tieback walls. Tieback walls that are permanent earth-retaining structures must remain serviceable throughout their economic life (Serviceability Performance Objective). In addition, the project design may require stringent displacement and settlement control (Displacement Control Performance Objective) to prevent damage to nearby structures, transportation systems, other infrastructure systems, and utilities. This may be a performance objective for both temporary and permanent tieback wall systems.

\subsubsection{Collapse prevention}

The objective of collapse prevention (CP) is to keep the tieback wall system from reaching a limit state that could result in failure. This is in part accomplished by the use of short- and long-term design loadings and load combinations that reduce the risk of overload during the life of the project to acceptable levels. It is also accomplished by using appropriate allowable stress design (ASD) procedures, and/or ultimate strength design (USD) procedures, for the analysis.

In ASD, the stresses at service load conditions are kept to a level that will provide a margin of safety sufficient to meet $\mathrm{CP}$ performance objectives. In USD, factored loads and nominal material strengths are used to provide the margin of safety necessary to meet CP performance objectives. Various potential structural and stability failure conditions for which a limit state analysis is required are illustrated in Figure 3.12. 


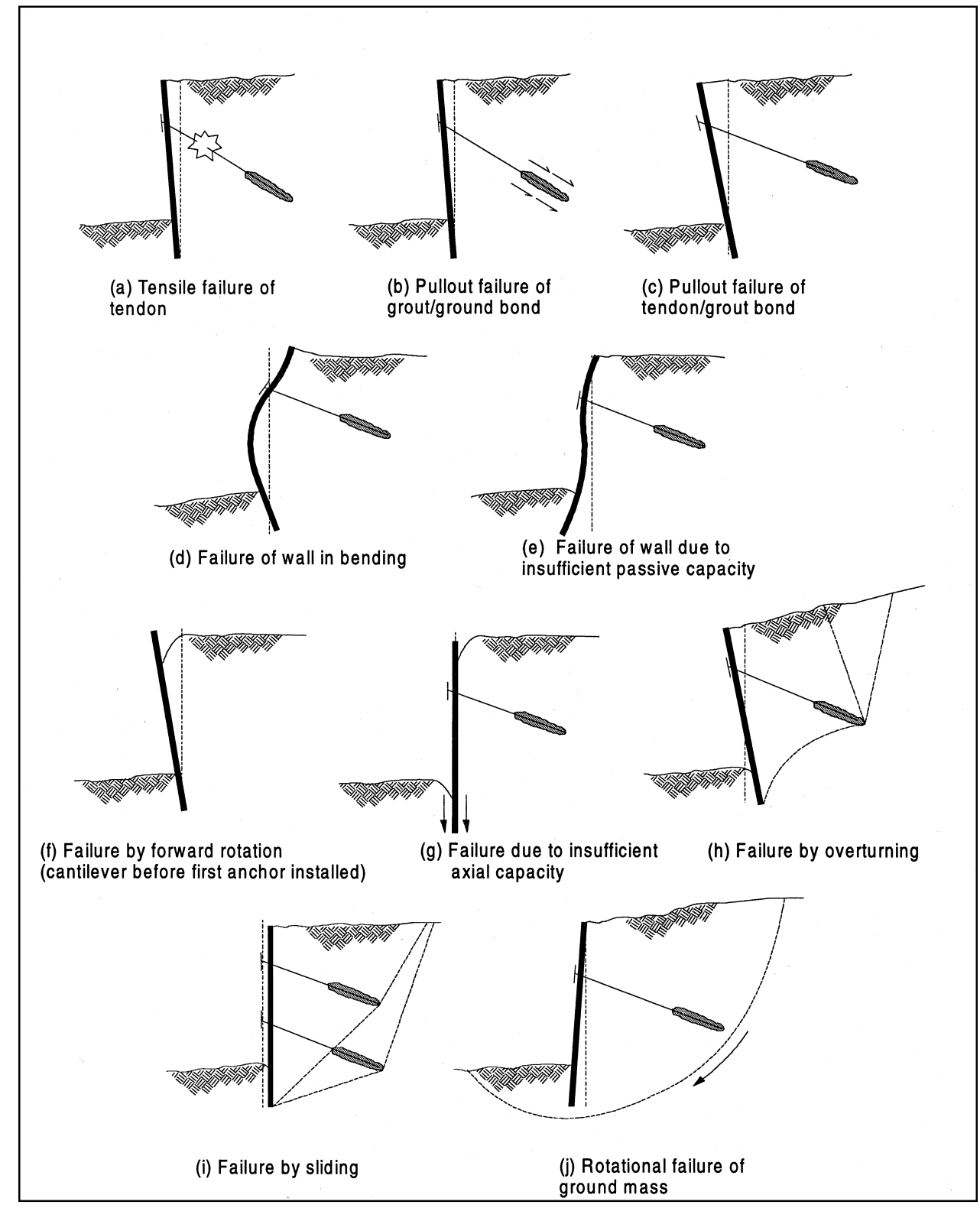

Figure 3.12. Potential failure conditions to be considered in design of anchored walls (after Figure 11; Sabatini, Pass, and Bachus 1999)

\subsubsection{Serviceability performance}

Serviceability performance is needed to ensure that the project will function as intended throughout its life. Deterioration of the anchors, soldier beams, wales, and other steel structural elements due to corrosion must be prevented. Excessive displacements that could lead to cracking and spalling of concrete, to deterioration of joints and waterstops, and to unacceptable visual effects must be prevented. Serviceability performance objectives are met by using ASD procedures, by limiting displacements to acceptable levels, by providing suitable corrosion protection, by controlling seepage and seepage pressures, and by controlling cracks in reinforced concrete lagging and facings. 


\subsubsection{Displacement control performance}

Although displacement control is part of the serviceability performance objective, in certain instances, displacement control in excess of that required for normal serviceability is required to protect structures and utilities adjacent to the excavation from damage. Since displacement control for the damage control performance objective is more stringent than that required for normal serviceability, it has been assigned a separate performance objective designation.

\subsection{Design Policy}

Design based on limit states using loads increased by load factors and strengths reduced by strength reduction factors $(\phi)$ is now the Corps-preferred method of design (EM 1110-2-2105, HQDA 1994). For steel structures, this approach is referred to as load and resistance factor design and, for concrete, as USD. With respect to tieback wall structural systems, however, the state of the practice has been to use ASD for the steel components (i.e., sheetpiling, soldier beams, and whales) and USD for concrete components (i.e., secant piles, continuous walls, lagging, and facing systems). Structural design state of the practice as presented herein therefore assumes that ASD will be used for steel components and USD for reinforced concrete components.

\subsection{Combination of Loads}

\subsubsection{General}

Combination of loads should be in accordance with the provisions of Standard No. 7-95 (ANSI/ASCE 1995), unless the excavation is for a highway or railway. The use of ANSI/ASCE (1995) endorses the concept of similar load factors for all materials. As such, the USD provisions cited herein as the state of the practice for reinforced concrete structures differ from those presented in EM 1110-2-2104 (HQDA 1992). For highway excavations the basic load combinations should be in accordance with the American Association of State Highway and Transportation Officials" "Standard Specifications for Highway Bridges" (AASHTO 1996). For railway excavations, the basic loading combinations should be in accordance with American Railway Engineering Association (AREA) specifications. Loads and load combinations for all stages of construction, as well as for all operating conditions, should be considered. The loads used for the design and evaluation of tieback wall systems are covered in Chapter 5.

\subsubsection{Allowable strength design of steel components}

In accordance with basic ASD provisions of ANSI/ASCE (1995), loads on tieback wall systems are considered to act in the combination given below. Effects of one or more loads not acting should also be investigated. 


$$
D+L+H
$$

where

$D=$ dead load

$L=$ live load

$H=$ load due to the weight and lateral pressure of soil and water in soil

It should be noted that the effects of earthquake loads are not addressed in this document, and therefore the loading combinations involving earthquake effects are not presented.

\subsubsection{Ultimate strength design of reinforced concrete components}

In accordance with basic USD provisions of ANSI/ASCE 7-95, reinforced concrete components are to be designed so that their strength equals or exceeds the effects of the factored loads in the following combination:

$$
1.2 D+1.6(L+H)
$$

For extreme loads, or loads of short duration such as barge impact loads, it is often the practice to reduce the load factor applied to the combination of effects involving live load, soil, and water. Using a reduction of 25 percent, the above load factor equation becomes

$$
1.2 D+1.2(L+H)
$$

\subsubsection{Special provisions for hydraulic structures}

Performance standards with respect to durability, maintainability, and operability are more stringent for hydraulic structures than for other conventional structures. Hydraulic structures are structures that are subjected to submergence, or wave action. Permanent tieback walls that serve as guide or guard walls for navigation lock projects are considered to be hydraulic structures. Reinforced concrete hydraulic structures under usual loading conditions are designed to keep the stresses in the reinforcement at low levels to prevent cracking and deterioration of the concrete. This is accomplished through the use of a hydraulic factor $\left(H_{f}\right)$. The hydraulic factor is equal to 1.3 and is applied to the USD loading combination above to provide the following loading combination for use in the design of those permanent tieback wall systems that serve as hydraulic structures:

$$
1.2 H_{f} D+1.6 H_{f}(L+H)
$$

Since the steel components of tieback wall systems that serve as hydraulic structures are designed by ASD methods, the approach to serviceability is one that reduces the allowable stress permitted for conventional tieback wall 
structures. To provide the desired durability, maintainability, and operability, the allowable stress permitted for hydraulic structures is set equal 0.83 times $(5 / 6)$ that allowed by the American Institute of Steel Construction, Inc. (AISC)-ASD.

\subsection{Applicable Design Codes and Standards}

\subsubsection{General}

Design codes and standards applicable to the design of tieback wall systems are listed in Appendix A. Design codes and standards applicable to the design and construction of the five types of walls covered by this report are listed in Tables 3.1-3.5. State-of-the-practice procedures for determining soil loads; for determining support system loads, moments, and shears; and for evaluating ground mass stability are covered in this report and in Sabatini, Pass, and Bachus (1999). Steel components of tieback wall systems are generally designed in accordance with the ASD provisions of AISC. The AISC provisions apply to drilled-in soldier beams, sheetpiling, and wales. However, when soldier beams are driven, the allowable stresses in the lower region of the soldier beam should be lower than those permitted by AISC. Allowable stresses for the lower regions of driven soldier beams can be found in EM 1110-2-2906 (HQDA 1991). Reinforced concrete elements of tieback wall systems are generally designed in accordance with the USD provisions of the American Concrete Institute's ACI 318. Prestressed tiebacks are designed in accordance with recommendations of the Post-Tensioning Institute's (PTI) "Recommendations for Prestressed Rock and Soil Anchors" and ACI 318. The design recommendations described in paragraph 3.8.2 are intended for tieback walls that must comply with Corps standards. Tieback walls supporting highway excavations should be designed in accordance with the applicable provisions of the AASHTO. Walls supporting railway excavations should be designed in accordance with the applicable provisions of AREA. Additional information related to the design of the various tieback wall features is provided in Chapter 8.

\begin{tabular}{|l|l|l||}
\hline \hline $\begin{array}{l}\text { Table 3.1 } \\
\text { Design Codes and Standards-Vertical Sheet-Pile System with } \\
\text { Wales and P-T Tieback Anchors }\end{array}$ \\
\hline \hline Structural Element & Design Code or Standard & Material Standard \\
\hline \hline Steel sheetpiling & $\begin{array}{l}\text { EM 1110-2-2504 } \\
\text { Design of Sheet Pile Walls }\end{array}$ & $\begin{array}{l}\text { ASTM A328 } \\
\text { ASTM A572 } \\
\text { ASTM A690 }\end{array}$ \\
\hline Wales & $\begin{array}{l}\text { EM 1110-2-2105 } \\
\text { Design of Hydraulic Steel Structures } \\
\text { AISC Manual of Steel Construction } \\
\text { Allowable Stress Design }\end{array}$ & $\begin{array}{l}\text { ASTM A36 } \\
\text { ASTM A572 }\end{array}$ \\
\hline Tieback anchors & $\begin{array}{l}\text { PTI Post-Tensioning Manual } \\
\text { PTI Recommendations for Prestressed } \\
\text { Rock and Soil Anchors }\end{array}$ \\
\hline
\end{tabular}




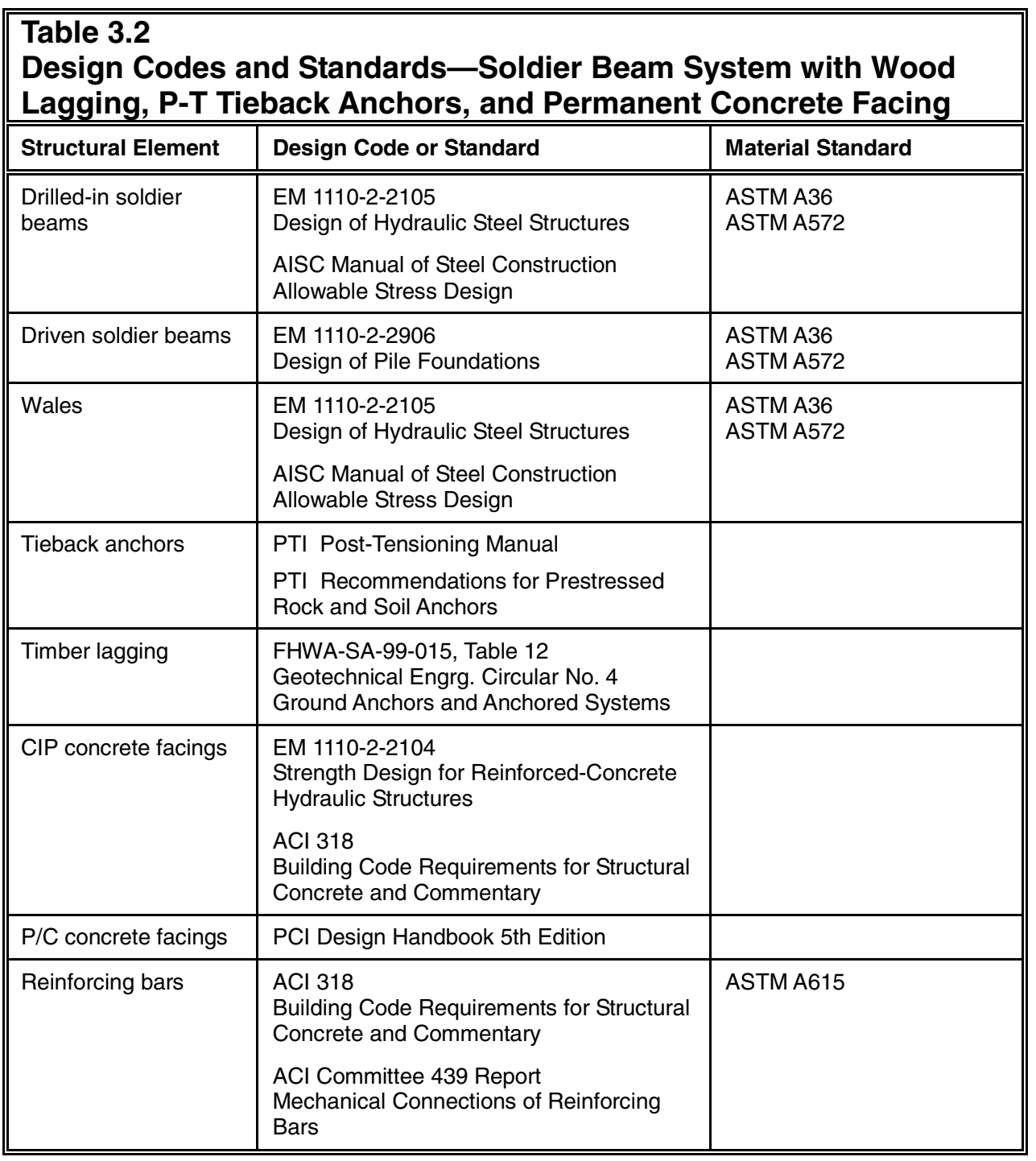




\begin{tabular}{|c|c|c|}
\hline \multicolumn{3}{|c|}{$\begin{array}{l}\text { Table } 3.3 \\
\text { Design Codes and Standards-Secant Cylinder Pile System with } \\
\text { P-T Tieback Anchors }\end{array}$} \\
\hline Structural Element & Design Code or Standard & Material Standard \\
\hline $\begin{array}{l}\text { Soldier beam cylinder } \\
\text { piles and lagging piles }\end{array}$ & $\begin{array}{l}\text { EM 1110-2-2104 } \\
\text { Strength Design for Reinforced-Concrete } \\
\text { Hydraulic Structures } \\
\text { ACI } 318 \\
\text { Building Code Requirements for Structural } \\
\text { Concrete and Commentary } \\
\text { ACI Committee } 336 \text { Report } \\
\text { Standard Specification for the Construction of } \\
\text { Drilled Piers }\end{array}$ & \\
\hline Tieback anchors & $\begin{array}{l}\text { PTI Post-Tensioning Manual } \\
\text { PTI Recommendations for Prestressed } \\
\text { Rock and Soil Anchors }\end{array}$ & \\
\hline Reinforcing bars & $\begin{array}{l}\text { ACI } 318 \\
\text { Building Code Requirements for Structural } \\
\text { Concrete and Commentary } \\
\text { ACI Committee } 439 \text { Report } \\
\text { Mechanical Connections of Reinforcing Bars }\end{array}$ & ASTM A615 \\
\hline
\end{tabular}

\begin{tabular}{|c|c|c|}
\hline \multicolumn{3}{|c|}{$\begin{array}{l}\text { Table } 3.4 \\
\text { Design Codes and Standards-Continuous Concrete Slurry Wall } \\
\text { System with P-T Tieback Anchors }\end{array}$} \\
\hline Structural Element & Design Code or Standard & Material Standard \\
\hline $\begin{array}{l}\text { Concrete slurry trench } \\
\text { wall }\end{array}$ & $\begin{array}{l}\text { EM 1110-2-2104 } \\
\text { Strength Design for Reinforced-Concrete } \\
\text { Hydraulic Structures } \\
\text { ACI } 318 \\
\text { Building Code Requirements for Structural } \\
\text { Concrete and Commentary } \\
\text { ACI Committee } 336 \text { Report } \\
\text { Standard Specification for the Construction of } \\
\text { Drilled Piers }\end{array}$ & \\
\hline Tieback anchors & $\begin{array}{l}\text { PTI Post-Tensioning Manual } \\
\text { PTI Recommendations for Prestressed } \\
\text { Rock and Soil Anchors }\end{array}$ & \\
\hline Reinforcing bars & $\begin{array}{l}\text { ACI } 318 \\
\text { Building Code Requirements for Structural } \\
\text { Concrete and Commentary } \\
\text { ACI Committee } 439 \text { Report } \\
\text { Mechanical Connections of Reinforcing Bars }\end{array}$ & ASTM A615 \\
\hline
\end{tabular}




\begin{tabular}{||l|l|l||}
\hline \multicolumn{2}{|l||}{ Table 3.5 } \\
Design Codes and Standards-Soldier Beam Tremie Concrete \\
System with P-T Tieback Anchors
\end{tabular}

\subsubsection{Special provisions of ASD applicable to tieback wall systems}

For Corps structures it is common practice to allow stress increases for unusual and extreme load conditions. An increase of 33 percent is permitted for unusual loading conditions, and an increase of 50 percent is permitted for extreme loading conditions (EM 1110-2-2504, HQDA 1994). Procedures that use apparent pressure diagrams to determine bending moments in soldier beams of flexible tieback wall systems are considered to be conservative. Therefore, with respect to temporary wall systems, it is considered acceptable to increase permissible stresses in flexure by 10 to 30 percent (ASCE 1997) or to adopt a reduction in the design loads.

\subsubsection{Anchors}

In accordance with the PTI's "Post-Tensioning Manual," the anchor bar or tendon size is determined such that the design load for the anchor does not exceed 60 percent of the guaranteed ultimate tensile strength of the bar or tendon. Lateral earth pressures based on apparent pressure diagrams in ASD loading combinations are used to determine the anchor design load. 


\subsubsection{Anchor bond lengths}

The anchor bond length should have the capacity to develop the anchor design load, determined as described above, with a minimum factor of safety of 2 . The factor of safety may be increased to account for site-specific conditions at the discretion of the designer.

\subsubsection{External stability}

The anchored wall should be stable for all potential failure planes with a factor of safety of 1.3 or more. The factor of safety may be increased to account for site-specific conditions at the discretion of the designer.

\subsubsection{Axial capacity of soldier beams}

Soldier beams should have sufficient axial capacity to develop axial loads due to all dead loads, live loads, and earth loads, including the vertical component of the anchor design load. Axial capacities of driven soldier beams can be determined in accordance with the provisions of EM 1110-2-2906 (HQDA 1991) or as indicated in Chapter 8. Axial capacities of driven soldier beams can be determined as indicated in Chapter 8 . Minimum factors of safety with respect to the maximum axial design load are generally as specified in EM 1110-2-2906.

\subsubsection{Tieback wall toe capacity}

The capacity of the tieback wall toe can be determined in accordance with the procedures described in Chapter 8. The minimum factor of safety with respect to the maximum toe design load (ASD) should be 1.5 or more. The factor of safety may be increased to account for site-specific conditions at the discretion of the designer. 


\section{Geotechnical Investigations and Testing}

\subsection{Objectives}

The primary objective of the geotechnical investigation for tieback wall design is to determine the engineering properties of the foundation materials in order to estimate wall loads and determine anchor requirements. In addition, investigations are needed to determine soil and rock stratigraphy to identify

- Weak soil and rock layers that are susceptible to sliding.

- Highly compressible materials that are susceptible to creep.

- Obstructions that can impede drilling, grouting, and wall installation.

- Groundwater tables.

- Drainage characteristics.

- Corrosion potential.

- Deformation characteristics of soils and rock for Winkler and FEM analyses.

- Capable anchorage zones.

- Required anchor development length, bond characteristics, and grouting methods.

Geotechnical investigations include field reconnaissance, laboratory testing, and in situ testing. Detailed information regarding geotechnical investigations can be found in HQDA Engineer Manuals 1110-1-1804, 1110-1-1906, 1110-12908, and 1110-2-1906. 


\subsection{Subsurface Exploration and Site Characterization}

As described in Chapter 3 of Sabatini, Pass, and Bachus (1999), subsurface explorations should be planned to gain full and accurate information beneath and immediately adjacent to the tieback wall. Information on the subsurface soil and rock stratigraphy and groundwater conditions is typically obtained from subsurface investigations. Subsurface investigations involve soil borings and rock coring, may likely involve in situ soil and rock testing, and generally involve obtaining disturbed and undisturbed samples for laboratory testing.

Subsurface borings should be advanced at regular intervals along, behind, and in front of the proposed wall alignment. Typical boring spacing, in plan, is 15 to $30 \mathrm{~m}$ (50 to $100 \mathrm{ft}$ ) for soil-anchored walls and 30 to $60 \mathrm{~m}(100$ to $200 \mathrm{ft})$ for rock-anchored walls. The investigation program should extend a distance behind the wall sufficient to capture the soil and rock properties at potential anchor locations and along potential failure planes. Usually this is a distance equal to 1.5 times the wall height. Subsurface explorations commonly extend a distance in front of the wall equal to 0.75 the wall height and to a depth below top of wall equal to twice the wall height. The geologist must have the latitude to locate borings as necessary to define the subsurface profile in the best way possible. As a minimum, soil borings should be sufficient to identify

- Soft settlement-prone layers containing highly plastic clays, and organic soils susceptible to long-term creep.

- Cohesionless sands and silts that tend to cave in when exposed or when water is encountered.

- Cohesionless sands susceptible to liquefaction of vibration-induced settlements.

- Obstructions, boulders, and cemented layers that adversely affect anchorhole drilling, grouting, and wall construction.

\subsection{Testing of Foundation Materials}

As indicated in Chapter 3 of Sabatini, Pass and Bachus (1999), laboratory and field testing must be sufficient to identify and accurately characterize the engineering properties and behavioral characteristics of soil and rock materials. This is important since the soil and rock materials will determine the wall loading as well as the ability of the anchor-wall-soil system to meet performance objectives.

Physical testing should focus on estimating the unit weight and shear strengths of soil and rock materials at the site. Testing requirements will vary depending on the type of ground.

For coarse-grained soils, the particle size distribution should be determined for each stratum. Unit weights and angles of shearing resistance can be estimated 
from correlations to standard penetration resistance (SPT) values for various types of course-grained soils (Weatherby 1998).

For fine-grained soils (e.g. clays), the total and dry unit weight values are frequently determined from tests performed on high-quality undisturbed samples. Atterberg limits and natural moisture content for each soil stratum are determined in the laboratory. For permanent tieback wall systems involving fine-grained soils, both undrained and drained strength parameters should be obtained and the anchor-wall-soil system designed for both short-term (undrained) and long-term (drained) conditions. Undrained shear strengths are determined from unconfined compression tests or consolidated undrained triaxial compression tests. Drained strengths are determined using consolidated drained triaxial compression tests or consolidated undrained triaxial compression tests with pore water pressure measurements (Weatherby 1998). Prior to the availability of site-specific test results, preliminary estimates of the drained shear strength for normally consolidated clays can be made using Figure 35 of Weatherby (1998).

Consolidation testing is performed on overconsolidated clays to determine the overconsolidation ratio. Using the overconsolidation ratio and plasticity index for the soil, a drained friction angle can be estimated based on information presented in Figure 35 of Weatherby (1998).

The details and procedures for performing soils testing can be found in EM 1110-2-1906.

\subsection{In Situ Testing of Foundation Materials}

In situ testing, especially the Standard Penetration Test (SPT), is often used to estimate relative density, shear strength, and the suitability of ground in cohesionless soils. Other in situ testing procedures may be of use in estimating soil type, shear strength, compressibility, drainage efficiency, and elastic modulus. These include the Cone Penetration Test, Vane Shear Test, Pressuremeter Test, and Flat Plate Dilatometer Test. Relative density and gradation can be used to estimate the friction angle of cohesionless soils (Table 4.1). Relative density is a measure of how dense a sand is compared with its maximum density. 


\begin{tabular}{|c|c|c|c|c|c|c|}
\hline \multicolumn{7}{|c|}{$\begin{array}{l}\text { Table 4.1 } \\
\text { Effective Angle of Internal Friction of Sands, ф' (after EM 1110-1-1905, Table 3-1) }\end{array}$} \\
\hline \multicolumn{7}{|c|}{ a. $\phi$ as a Function of Relative Density and Gradation (data from Schmertmann 1978) } \\
\hline \multirow{2}{*}{$\begin{array}{l}\text { Relative } \\
\text { Density, } D_{r} \\
\text { (percent) }\end{array}$} & \multicolumn{2}{|c|}{ Fine-Grained } & \multicolumn{2}{|c|}{ Medium-Grained } & \multicolumn{2}{|c|}{ Coarse-Grained } \\
\hline & Uniform & Well-Graded & Uniform & Well-Graded & Uniform & Well-Graded \\
\hline 40 & 34 & 36 & 36 & 38 & 38 & 41 \\
\hline 60 & 36 & 38 & 38 & 41 & 41 & 43 \\
\hline 80 & 39 & 41 & 41 & 43 & 43 & 44 \\
\hline 100 & 42 & 43 & 43 & 44 & 44 & 46 \\
\hline \multicolumn{7}{|c|}{ b. $\phi$ as a Function of Relative Density and In Situ Soil Tests } \\
\hline \multirow[b]{2}{*}{ Soil Type } & \multirow[b]{2}{*}{$\begin{array}{l}\text { Relative } \\
\text { Density, } D_{r} \\
\text { (percent) } \\
\end{array}$} & \multirow{2}{*}{$\begin{array}{l}\text { Standard } \\
\text { Penetration } \\
\text { Resistance, } \\
N_{60} \text { (Terzaghi } \\
\text { and Peck } \\
1967)\end{array}$} & \multirow{2}{*}{$\begin{array}{l}\text { Cone } \\
\text { Penetration } \\
\text { Resistance qc, } \\
\text { ksf (Meyerhof } \\
\text { 1974) } \\
\end{array}$} & \multicolumn{3}{|c|}{ Friction Angle, $\phi^{\prime}$ (deg) } \\
\hline & & & & $\begin{array}{l}\text { Meyerhof } \\
(1974)\end{array}$ & $\begin{array}{l}\text { Peck, Hanson, } \\
\text { and } \\
\text { Thornburn } \\
\text { (1974) } \\
\end{array}$ & $\begin{array}{l}\text { Meyerhof } \\
(1974)\end{array}$ \\
\hline Very loose & $<20$ & $<4$ & --- & $<30$ & $<29$ & $<30$ \\
\hline Loose & $20-40$ & $4-10$ & $0-100$ & $30-35$ & $29-30$ & $30-35$ \\
\hline Medium & $40-60$ & $10-30$ & $100-300$ & $35-38$ & $30-36$ & $35-40$ \\
\hline Dense & $60-80$ & $30-50$ & $300-500$ & $38-41$ & $36-41$ & $40-45$ \\
\hline Very dense & $>80$ & $>50$ & $500-800$ & $41-44$ & $>41$ & $>45$ \\
\hline
\end{tabular}

\subsubsection{Relative density definition}

ASTM D 653 defines relative density as the ratio of the difference in void ratio of a cohesionless soil in the loosest state at any given void ratio to the difference between the void ratios in the loosest and in the densest states. Very loose sand has a relative density of 0 percent and 100 percent in the densest possible state. Extremely loose honeycombed sands may have a negative relative density.

\subsubsection{Relative density calculations}

Relative density $\left(D_{r}\right)$ can be calculated using standard test method ASTM D 4254 and the void ratio of the in situ cohesionless soil,

$$
\begin{aligned}
& D_{r}=\frac{e_{\text {max }}-e}{e_{\text {max }}-e_{\text {min }}} \cdot 100 \\
& e=\frac{G}{\gamma_{d}} \gamma_{w}-1
\end{aligned}
$$

where 


$$
\begin{aligned}
e_{\max } & =\text { reference void ratio of a soil at the minimum density } \\
e_{\min } & =\text { reference void ratio of a soil at the maximum density } \\
G & =\text { specific gravity } \\
\gamma_{\mathrm{d}} & =\text { dry density } \\
\gamma_{\mathrm{w}} & =\text { unit weight of water }
\end{aligned}
$$

The specific gravity of the mineral solids can be determined using standard test method ASTM D 854. The dry density of soils can be determined in situ using standard test method ASTM D 1556.

\subsection{Identifying Corrosive Soil Environments}

For permanent anchored systems the corrosion potential of the site must be evaluated as indicated in Chapters 3 and 6 of Sabatini, Pass and Bachus (1999). Although corrosion of anchor systems can occur by chemical attack, the most common form of corrosion is electrochemical. The electrochemical corrosion rate increases in the presence of soluble salts, especially sodium chloride. Other soluble salts such as perchlorates, acetates, and salts of halogens can also be corrosive to anchor systems. Hydrogen sulfide has also been cited as a cause of corrosion, as have stray currents.

The corrosion rate of steel in soil environments will decrease as the $\mathrm{pH}$ level of the groundwater increases. In general, ground environments may be classified as aggressive if any one of the following conditions is present in the ground or may be present during the service life of the ground anchor (PTI 1996):

- The $\mathrm{pH}$ of the soil or groundwater is less than 4.5.

- The resistivity of the ground is less than $2,000 \mathrm{ohm}-\mathrm{cm}$.

- Sulfides are present.

- Stray currents are present.

- The potential for corrosion or direct chemical attack has been observed in existing buried concrete structures located adjacent to the site.

The potential for corrosion due to soil and groundwater must be evaluated. Corrosion potential can be determined based on the results of the following tests:

- $\quad$ H test (ASTM G51, AASHTO T289).

- Electrical resistivity test (ASTM G57, AASHTO T288).

- Chloride content test (ASTM D512, AASHTO T291).

- $\quad$ Sulfate content test (ASTM D516, AASHTO T290). 


\section{RIGID Analysis Procedure}

\subsection{General}

The RIGID analysis terminology as well as the Winkler and FEM terminologies are in accordance with those used by Kerr and Tamaro (1990). In the RIGID method the wall is assumed to be elastic (with a constant EI) and continuous over fixed ground anchor supports. In general accordance with Kerr and Tamaro (1990), the salient features of the RIGID analysis are these:

- Assumes a fictitious support below subgrade at zero net pressure.

- Soldier beams, secant piles, slurry wall, sheet pile, etc., below this point are neglected in the analysis.

- Supports are presumed to be rigid, and movement prior to ground anchor installation is neglected.

- Loading can be trapezoidal (apparent pressure) or triangular.

- The loading used is considered a following load, and no effort is made to redistribute earth pressures as a result of wall movement.

- The model is reduced to an elastic beam that is continuous over rigid supports.

In practice, the procedures used to design flexible tieback wall systems differ from those used to design stiff tieback wall systems.

In general for the flexible wall systems, apparent pressure diagrams are applied to the tieback wall, and the anchor forces are determined by a tributary area method (Peck 1969; Sabatini, Pass, and Bachus 1999). Another form of the analysis allows the total load on the wall to be determined by Coulomb analysis or by sliding wedge analysis. The total load is then converted to a trapezoidal or triangular pressure diagram representing approximate actual earth pressures on the wall. Tieback anchor forces and wall bending moments for flexible wall systems are usually estimated using simple approximate procedures based on apparent pressure diagrams.

Displacements, earth pressures, and earth pressure distributions for stiffer tieback wall systems are, however, somewhat different from those of the more flexible systems. For the stiffer wall systems, triangular earth pressure distributions are generally used to evaluate anchor forces and wall bending 
moments, especially near the base of the wall where earth pressures are typically greater than those indicated by apparent pressure diagrams. In many instances, the earth pressure loads associated with various stages of construction are considered in the design of the stiffer wall systems.

\subsection{Apparent Versus Actual Earth Pressures}

As indicated in Chapter 3, the soil pressure envelopes, or apparent earth pressure diagrams developed by Peck (1969), and as presented in Peck, Hanson, and Thornburn (1974), were not intended to represent the real distribution of earth pressure, but instead constituted hypothetical pressures. These hypothetical pressures were a basis from which there could be calculated strut loads that might be approached but would not be exceeded in the actual cut. However, it has been noted (Liao and Neff 1990) that the apparent pressure diagram recommended by Peck (1969) provides a total pressure equal to $0.217 \gamma \mathrm{H}^{2},(\phi=30 \mathrm{deg})$, whereas the total active pressure is $0.167 \gamma \mathrm{H}^{2}$, and the total at-rest pressure (for $K_{o}=0.5$ ) is $0.250 \gamma \mathrm{H}^{2}$. This means that on an actual pressure basis, the total load on the wall is equal to something between active and at-rest pressures. For sand it is approximately equal to the total load obtained from limiting equilibrium analysis using a factor of safety of 1.3 on the soil shear strength. Liao and Neff (1990) also demonstrated that the trapezoidal earth pressure distributions proposed by Peck (1969) for dense sands and stiff fissured clays can theoretically reflect the shapes of the actual soil pressures behind tieback walls. However, it is more likely that nontriangular distributions will occur in overconsolidated dense sands and stiff to hard clays as shown in Figure 5.1c. The earth pressure distributions for loose sands and soft clays will tend to be more triangular in shape, as shown in Figure 5.1b.

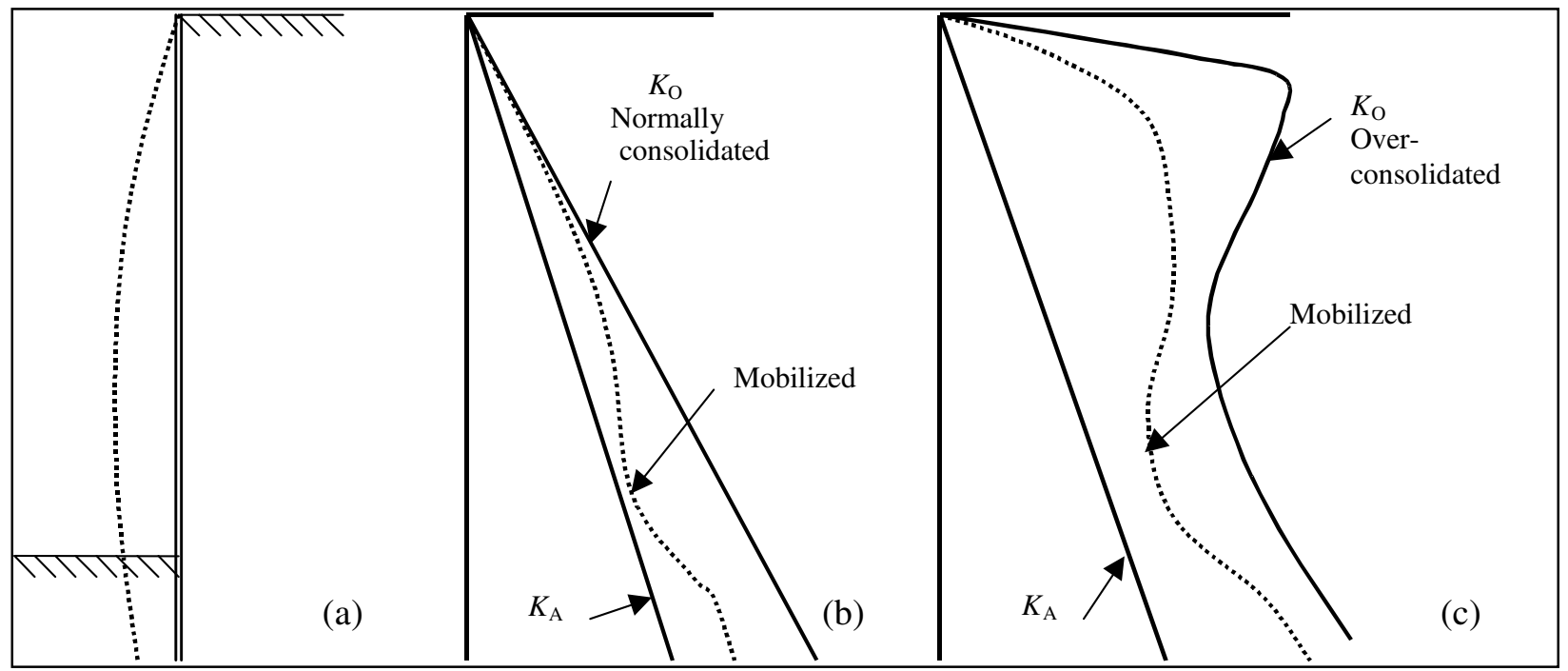

Figure 5.1. Schematic model for redistribution of lateral stress due to wall movement (after Liao and Neff 1990, Figure 5)

(a) Hypothesized movement

(b) Hypothesized stress - normally consolidated soil

(c) Hypothesized stress - overconsolidated soil 


\subsection{Earth Pressures}

\subsubsection{Background}

Apparent pressure diagrams were first developed by Terzaghi and Peck (1967) and by Peck (1969). These diagrams represent an envelope of pressures on braced wall systems back-calculated from the field measurements of strut loads. These apparent pressure diagrams, as discussed in Peck, Hanson and Thornburn (1974), are idealized in Figure 5.2. They were developed for homogeneous soil profiles representing

- Drained loadings in sand.

- Undrained loadings in stiff to hard fissured clays.

- Undrained loadings in soft to medium clays.

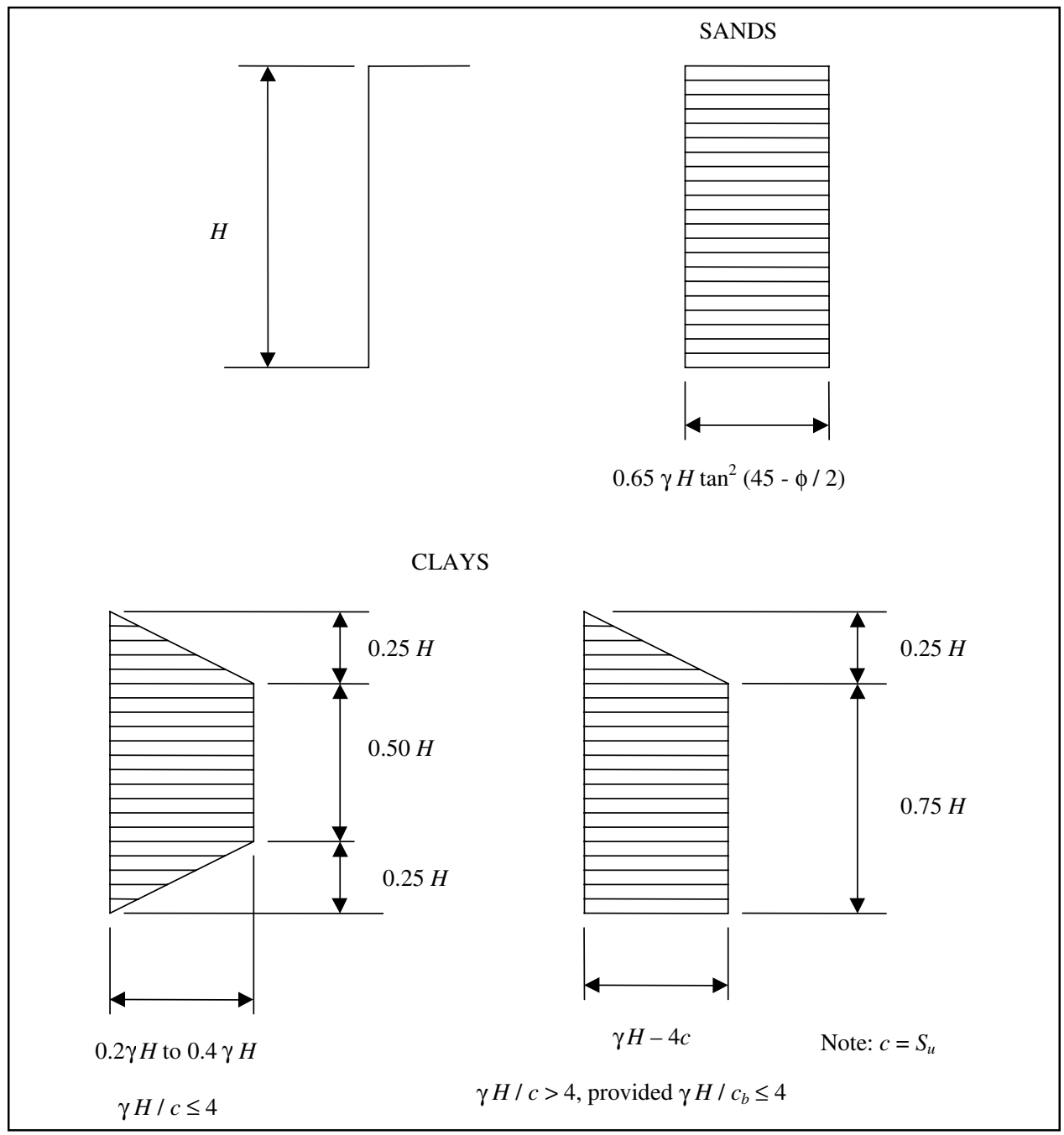

Figure 5.2. Apparent earth pressure diagrams (adapted from Figure 19, Weatherby 1998) 


\subsubsection{Modifications to Terzaghi and Peck apparent pressure diagrams}

Since 1969, modifications to the original Terzaghi and Peck apparent pressure diagrams have been proposed. Two notable modifications (Sabatini, Pass, and Bachus 1999) are described below:

- Henkel (1971) modified the equation used to calculate the maximum earth pressure ordinate for the Terzaghi and Peck (1967) soft to medium clay apparent pressure diagram. Henkel assumed a failure mechanism consistent with deep-seated movements for excavations in soft to medium clays that had not been previously used by Peck (1969). Backcalculated values of the active earth pressure coefficient for excavations in which deep-seated movements occurred indicated that Peck's method underpredicted the active pressure coefficient whereas Henkel's method more accurately predicted the active pressure coefficient.

- In Weatherby (1998), a variation in the distribution of earth pressure calculated from Terzaghi and Peck's (1967) apparent earth pressure diagram for sand and stiff to hard fissured clay is proposed. Weatherby (1998) notes that permanent ground anchors are seldom built in normally consolidated clay deposits. However, Weatherby includes an apparent pressure diagram for soft clays, which can be used in the design of tieback walls that are provided only for temporary support. This diagram is presented as Figure 5.6 of this report. The earth pressure for anchored walls with flexible wall elements is greatly influenced by the prestressing and lock-off procedure used for each anchor. Earth pressures concentrate at anchor locations. The apparent earth pressure diagram for anchored walls in sand and stiff to hard fissured clay requires that the location of the uppermost and lowermost anchor be known. Therefore, the distribution of earth pressure, in addition to being influenced by excavation depth (as in the case for Terzaghi and Peck apparent earth pressure diagrams), is also influenced by the location of the anchors.

The above recommendations are often incorporated into the RIGID analysis procedure for walls with homogeneous soil profiles. Additional modifications in the design and analysis procedure applicable to stiff walls (secant pile and continuous slurry trench walls) are discussed later. The use of the apparent pressure diagram approach in a RIGID analysis procedure permits relatively simple "hand calculations" of ground anchor loads and wall bending moments (see Figures 5.3 and 5.4). These figures are intended to represent a loading envelope that can be used to design ground anchor supports, soldier beams, and other tieback wall structural components for flexible wall systems for conditions reflecting the entire construction and in-service history. They do not represent loads that might exist on the wall at any one time. 


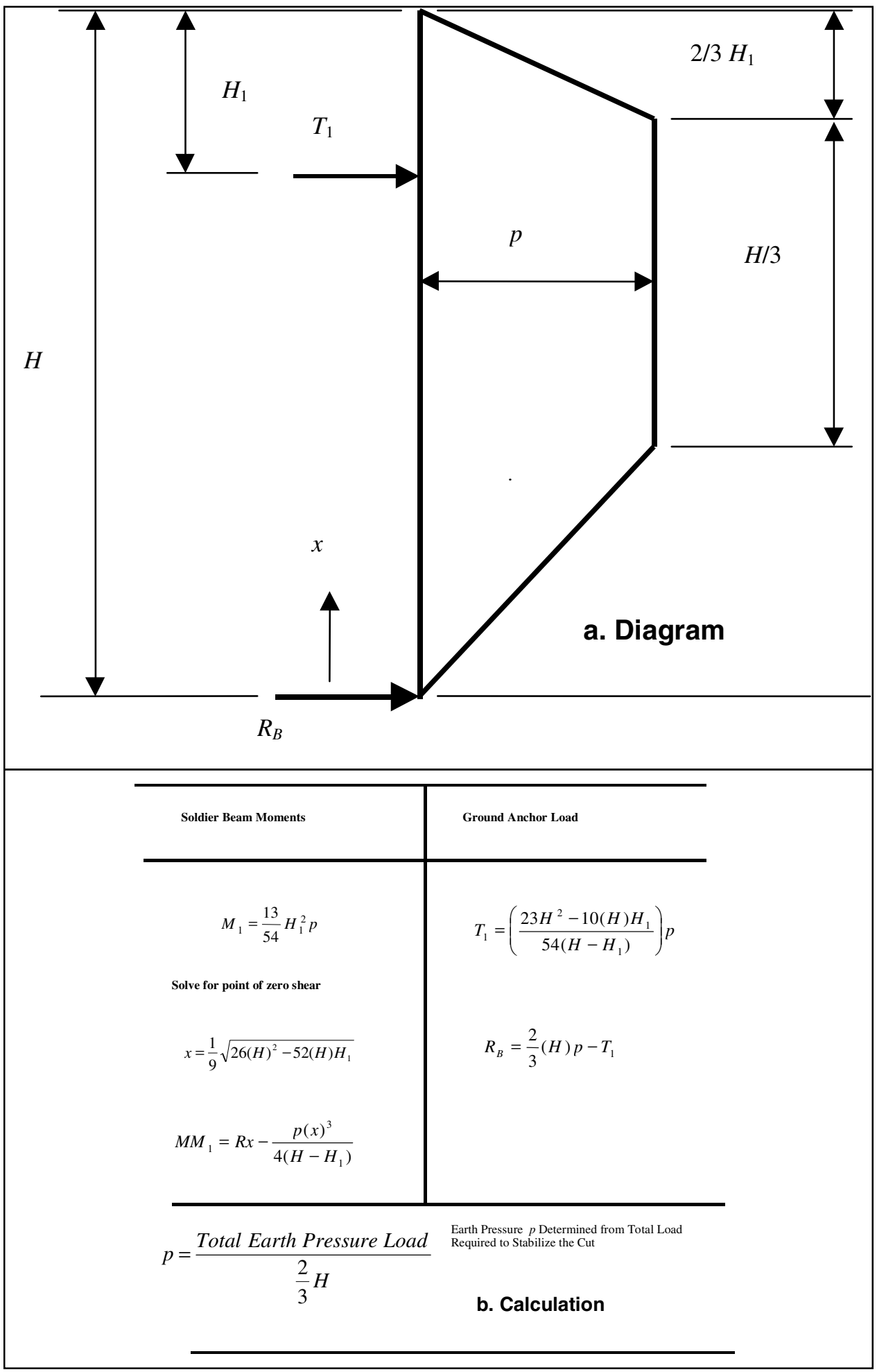

Figure 5.3. Recommended apparent earth pressure diagram formulas for wall supported by one row of anchors (for granular soils) (Figure 28, Weatherby 1998) 


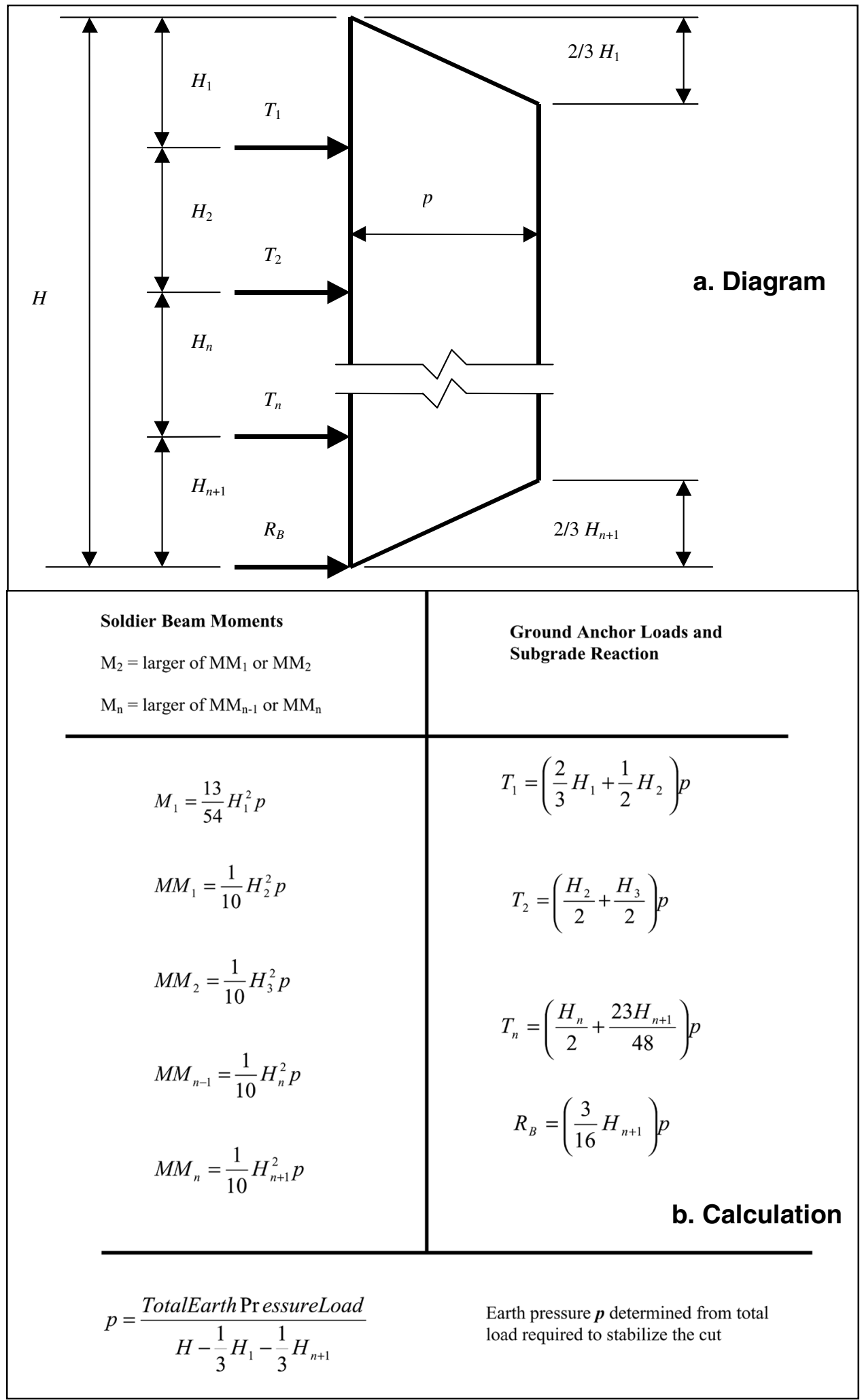

Figure 5.4. Recommended apparent earth pressure diagram formulas for wall supported by multiple rows of anchors (for granular soils) (adapted from Figure 29, Weatherby 1998). [Note: Errors in equation notation given in Weatherby 1998 (Figure 29) are corrected here.] 
Redistribution of earth pressures results in actual bending moments being less that those computed using apparent pressure diagram loadings (Mueller, Long, Weatherby, Cording, Powers, and Briaud 1998). Accordingly, Peck, Hanson, and Thornburn (1974) indicated that soldier beam moments might be computed using two-thirds of the apparent pressures. Consistently good results over many years have shown that, with respect to flexible wall systems, apparent earth pressure diagrams are suitable for determining anchor loads and are conservative in estimating bending moments (Weatherby 1998). For stiffer wall systems, a somewhat different approach has been used to determine bending moments. This approach is described in later paragraphs.

\subsubsection{Total load approach}

FHWA procedures (Sabatini, Pass, and Bachus 1999; Weatherby 1998) allow the apparent pressure diagrams to be constructed based on a "total load" approach. In this approach, factors of safety are applied to active limit state conditions to obtain the total design load. This total design load is then converted to an apparent pressure diagram, which is used as the basis for determining anchor loads and wall bending moments.

In practice, two safety factor approaches are used. In the first approach the total active load (determined by Rankine, Coulomb, or limiting equilibrium analysis) is increased by a factor of safety to obtain the total design load. This method is defined as the $F S_{\text {Load }}$ method since the factor of safety is applied to the active pressure load. In the second approach, the factor of safety is applied to the soil shear strength parameters $\left(c^{\prime}\right.$ and $\left.\phi^{\prime}\right)$. This method is defined as the $F S_{\text {Strength }}$ method. The $F S_{\text {Strength }}$ method is common to limiting equilibrium analysis and, unless otherwise indicated, is the method to be assumed with respect to the following discussions.

\subsubsection{Apparent earth pressure diagrams for coarse-grained soils}

Figures 5.3a and 5.4a show apparent pressure diagrams for coarse-grained soils constructed in accordance with new procedures developed by Weatherby (1998). The diagram in Figure 5.3a is for a flexible wall supported by one row of anchors, and the diagram in Figure 5.4a is for a flexible wall supported by multiple rows of anchors. The intensity of the pressure in these diagrams is calculated from the total lateral earth load.

Total lateral earth load can be the total load from Terzaghi and Peck's sand diagram $\left(0.65 \mathrm{~K}_{\mathrm{a}} \gamma \mathrm{H}^{2}\right)$ or the load determined from a limiting equilibrium analysis. These pressure diagrams are called "nonsymmetrical trapezoidal pressure diagrams." Additional discussion about nonsymmetrical earth pressure diagrams is contained in Weatherby, Chung, Kim, and Briaud (1998). The earth pressure in these diagrams increases to a maximum at a depth equal to two-thirds the distance to the upper anchor. 
For a wall supported by one row of anchors, the maximum pressure continues downward for a distance equal to one-third the height of the wall. Below that depth, the pressure decreases linearly to zero at the bottom of the excavation. The total lateral load is $0.65 K_{a} \gamma H^{2}$ (the total load from the Terzaghi and Peck diagram), and the intensity of the maximum pressure on the one-row wall is approximately $K_{a} \gamma H$.

For a wall supported by multiple levels of ground anchors, the maximum earth pressure continues to a point below the lowest support equal to one-third the distance from the lower support to the bottom of the excavation. From there the pressure decreases linearly to zero at the bottom of the excavation. The total load for the multitiered wall is the same as for the one-tier wall, $0.65 K_{a} \gamma H^{2}$. The nonsymmetrical trapezoid is considered to be more appropriate than the rectangular diagram for the design of flexible tieback walls since

- Measurements show that arching concentrates the earth pressures at the ground anchor locations.

- The earth pressures in a sand deposit must be zero at the ground surface.

- Actual earth pressures increase from the ground surface to the ground anchor location.

- Bending moments predicted using the nonsymmetrical diagram fit measured results for flexible tieback wall systems better than those predicted by other apparent pressure diagrams.

- Ground anchor loads determined from the nonsymmetrical trapezoid diagram are similar to those determined using other apparent pressure diagrams.

Equations for determining the ground anchor loads and soldier beam bending moments are presented in Figures 5.3b and 5.4b. These equations use the tributary area method for determining ground anchor loads. The equation for the bending moment at the upper anchor is determined by summing moments about the anchor. Bending moment equations below the upper support are empirical and are based on the maximum earth pressure intensity and the span distance between anchors. These moments can represent either maximum positive or maximum negative bending moment and, as such, the location of the point of maximum moment is unidentified.

As stated previously, the total lateral earth load from Terzaghi and Peck's sand diagram is $0.65 \mathrm{~K}_{\mathrm{a}} \gamma \mathrm{H}^{2}$. Total lateral earth load can be expressed as an EPF (earth pressure factor) $\left(0.65 \mathrm{~K}_{\mathrm{a}} \gamma\right)$ times the square of the wall height. Figure 5.5 is a plot of EPFs for the coarse-grained soils in Table 5.1. Using values for the effective angle of internal friction between 27 and $38 \mathrm{deg}$ and total unit weights between 95 and 134 pcf, Weatherby shows that the EPF has a narrow range between 20 and 24 pcf (see Table 5.1). The narrow range of EPFs reflects the relationship among friction angle, total unit weight, and density. When the deposit is dense, the friction angle and the total unit weight are high. The opposite is true for loose deposits. Since the EPFs were developed from Terzaghi 
and Peck's sand diagram, they include a factor of safety of about 1.3 applied to the soil shear strength. Figure 5.5 can be used to determine the total lateral earth pressure load to be used to develop the pressure for the nonsymmetrical earth pressure diagram.

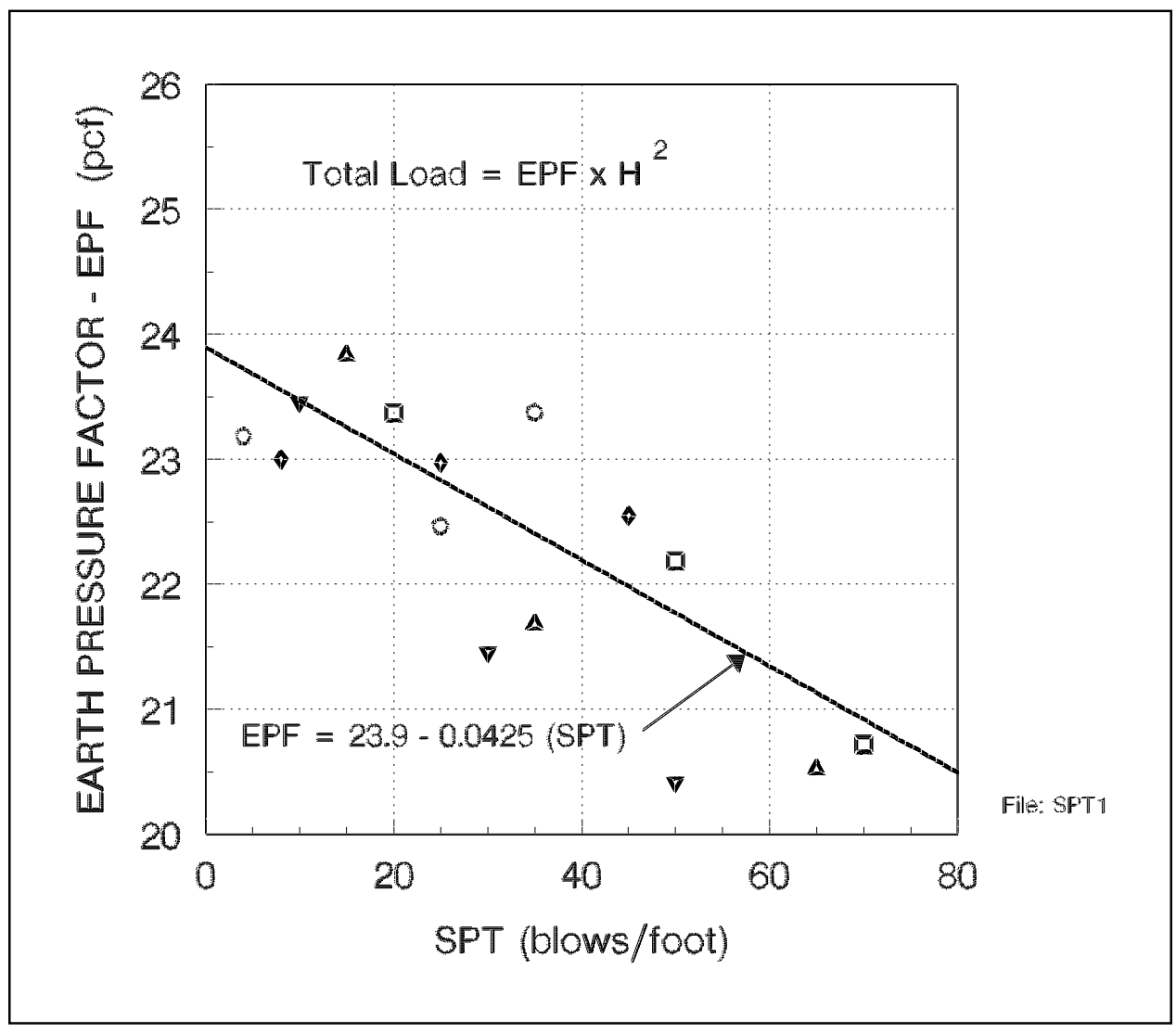

Figure 5.5. Earth pressure factors (EPF) as a function of standard penetration resistance - symbols represent plot of Table 5.1 data (adapted from Figure 30, Weatherby 1998) 


\begin{tabular}{|c|c|c|c|c|c|c|}
\hline \multicolumn{7}{|c|}{$\begin{array}{l}\text { Table } 5.1 \\
\text { Earth Pressure Factors for Typical Coarse-Grained Soils (after Weatherby 1998a, Table } 8\end{array}$} \\
\hline Soil Type & $\begin{array}{l}\text { Relative } \\
\text { Density }\end{array}$ & $\mathrm{SPT}^{1}$ (blows/ft) & $\begin{array}{l}\phi \\
\text { (deg) }\end{array}$ & $\mathbf{K}_{\mathbf{A}}$ & $\begin{array}{l}\text { Total Weight } \gamma \\
\text { (pcf) }\end{array}$ & EPF (pcf) \\
\hline \multirow{3}{*}{$\begin{array}{l}\text { GP } \\
\text { (Poorly graded } \\
\text { gravel, gravel } \\
\text { sand mixture) }\end{array}$} & Dense & 70 & 38 & 0.238 & 134 & 20.72 \\
\hline & Medium dense & 50 & 35 & 0.271 & 126 & 22.19 \\
\hline & Loose & $<20$ & 32 & 0.307 & 117 & 23.37 \\
\hline \multirow{3}{*}{$\begin{array}{l}\text { SW } \\
\text { (Well graded } \\
\text { sand, gravelly } \\
\text { sand) }\end{array}$} & Dense & 65 & 37 & 0.249 & 127 & 20.52 \\
\hline & Medium dense & 35 & 34 & 0.283 & 118 & 21.68 \\
\hline & Loose & $<15$ & 30 & 0.333 & 110 & 23.83 \\
\hline \multirow{3}{*}{$\begin{array}{l}\text { SP } \\
\text { (Poorly graded } \\
\text { sand, gravelly } \\
\text { sand) }\end{array}$} & Dense & 50 & 36 & 0.260 & 121 & 20.42 \\
\hline & Medium dense & 30 & 33 & 0.295 & 112 & 21.46 \\
\hline & Loose & $<10$ & 29 & 0.347 & 104 & 23.46 \\
\hline \multirow{3}{*}{$\begin{array}{l}\text { SM } \\
\text { (Silty sand, sand } \\
\text { silt mixture) }\end{array}$} & Dense & 45 & 35 & 0.271 & 128 & 22.55 \\
\hline & Medium dense & 25 & 32 & 0.307 & 115 & 22.97 \\
\hline & Loose & $<8$ & 29 & 0.347 & 102 & 23.00 \\
\hline \multirow{3}{*}{$\begin{array}{l}\text { ML } \\
\text { (Silt with little or } \\
\text { no plasticity) }\end{array}$} & Dense & 35 & 33 & 0.295 & 122 & 23.38 \\
\hline & Medium dense & 25 & 31 & 0.320 & 108 & 22.47 \\
\hline & Loose & $<4$ & 27 & 0.376 & 95 & 23.19 \\
\hline
\end{tabular}

Limiting equilibrium analyses can also be used to determine the pressure for the nonsymmetrical trapezoid (Weatherby 1998). This is accomplished by determining the total earth pressure load corresponding to a factor of safety of 1.3 on the soil shear strength. A factor of safety of 1.3 assumes that wall deflections are tending toward deflections consistent with active limit state conditions. Higher factors of safety (1.4 to 1.5 ) would be used for conditions representative of at-rest earth pressure conditions. The total lateral earth pressure load (horizontal component) determined from the limiting equilibrium analysis can then be used to calculate the maximum apparent earth pressure intensity using the equations in Figures 5.3b and 5.4b.

\subsubsection{Apparent earth pressure diagrams for stiff clays}

Report FHWA-RD-97-130 (Weatherby 1998) recommends a nonsymmetrical apparent pressure diagram for stiff clays that is identical in shape to the one recommended for granular soils (Figures 5.3a and 5.5a) when undrained (short-term) conditions exist. Weatherby (1998) also uses the abovementioned diagrams for long-term drained conditions in stiff clays. This FHWA report recommends an EPF of 20 pcf unless experience suggests that a higher EPF should be used. In addition, the report indicates that the transition from a stiff clay apparent pressure diagram to a soft to medium clay diagram does not occur at a unique undrained shear strength. For a given wall height or excavation depth, $H$, the undrained shear strength of the soil, $s_{u}$, should be greater than 
$\frac{H}{4}(\gamma-22.857)$ in order to use the apparent pressure diagram recommended above for stiff clays.

Limiting equilibrium analyses cannot be used to calculate the total lateral earth load for a wall built in stiff clay because loads on walls in a stiff clay deposit correspond to a quasi-elastic state instead of a state of limiting equilibrium under short-term (undrained) loading conditions. Limiting equilibrium analyses for stiff clays suggest that the ground is strong enough to support itself, or they give loads that are too low based on measured strut loads.

Weatherby (1998) indicates that long-term (postconstruction) earth pressures for stiff fissured clays may depend on the drained shear strength of the soil, and could result in a higher total load than that determined using stiff clay apparent earth pressure diagrams based on undrained (short-term) conditions. Liao and Neff (1990) recognized that, for clays, the apparent increase of strut of tieback loads with time can probably, in most cases, be explained in terms of negative pore-water pressure dissipation leading to increases in total stress imposed on the excavation wall. Designers of walls in clay need to carefully consider these issues to ensure that the greatest potential total load has been selected for use in the design and evaluation of tieback wall systems. Earth pressures for long-term conditions should consider drained shear strength conditions and reasonable pore water pressures. Permanent tieback walls should be designed to support both short- and long-term earth pressure conditions.

Sabatini, Pass, and Bachus (1999) recommend that the total load for stiff to hard fissured clays be based on previous experience with excavations constructed in similar deposits. These investigators suggest that for temporary wall loadings (short term), the total load will vary between $0.17 \gamma \mathrm{H}^{2}$ and $0.33 \gamma \mathrm{H}^{2}$ (see Table 5 of Sabatini, Pass, and Bachus 1999). They also suggest that, for permanent conditions in homogeneous soil profiles, the total load will be approximately equal to $0.65 K_{A} \gamma H^{2}$, where $K_{A}$ is based on the drained friction angle of the clay soil.

\subsubsection{Apparent earth pressure diagrams for soft to medium clays}

Temporary and permanent ground anchor walls in soft to medium clay must resist the short-term lateral earth pressures determined using undrained shear strengths and total unit weights. Weatherby (1998) notes that permanent ground anchors are seldom built in normally consolidated clay deposits. This section (taken from Weatherby 1998) discusses the determination of these pressures when competent ground is at or near the bottom of the excavation. As cited above for ground anchor walls in stiff clays, the long-term loading determined using drained shear strengths, effective unit weights, and pore pressures may be greater than the loads determined for short-term conditions using undrained shear strengths and total unit weights. In addition, the concerns expressed by Liao and Neff (1990) with respect to the dissipation of pore pressures in the long term and the result this has on the total stress applied to the wall need to be considered in the long-term evaluation of tieback walls constructed in soft to medium clays. 
Apparent pressure diagrams for soft to medium clays based on drained shear strengths are addressed in subsequent sections.

Terzaghi and Peck's soft to medium clay diagram forms the basis for determining the lateral earth pressures based on the undrained strength of the clay (see Figure 5.2). In Figure 5.2 the cohesive strength, $c$, is equal to the undrained shear strength, $S_{u}$. Earth pressure factors for clay are equal to $0.875(1-4$ $\left.S_{u} / \gamma H\right) \gamma$, with the total lateral earth load equal to $0.875 H^{2}\left(1-4 S_{u} / \gamma H\right) \gamma$. The total lateral earth load is distributed to the wall using an earth pressure diagram with the same shape as Terzaghi and Peck's soft to medium clay diagram. Figure 5.6 shows the earth pressure diagram and contains an equation for determining the earth pressure intensity based on the total earth pressure load.

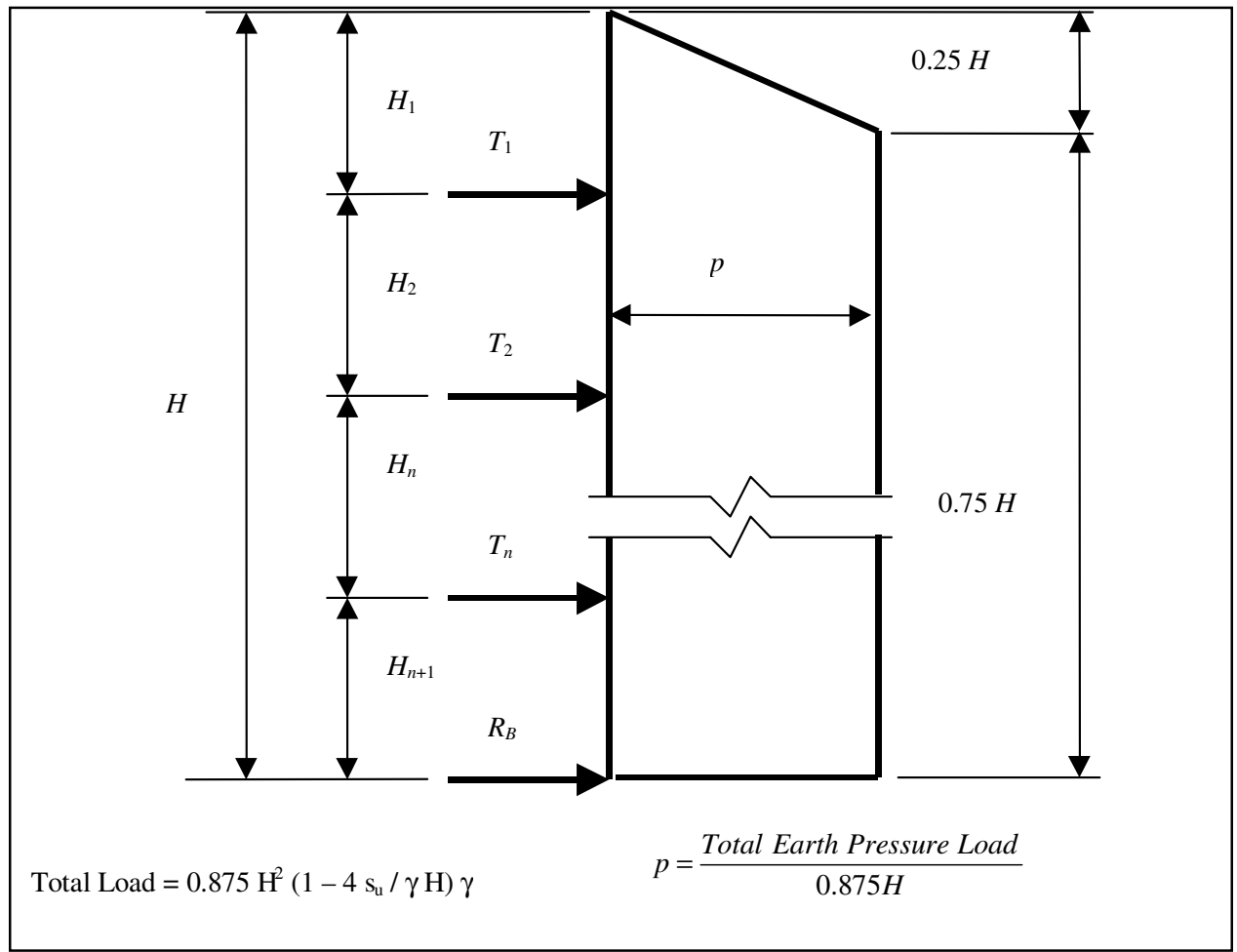

Figure 5.6. Soft to medium clays (used for temporary support only) recommended apparent earth pressure diagram for wall supported by multiple rows of anchors (adapted from Figure 30, Weatherby 1998)

Figure 5.6 illustrates a wall with multiple tieback anchors. The shape of the earth pressure diagram for a one-tier tieback wall system is identical to that for the multitiered system. Anchor loads are often determined by the tributary area method. The negative moment at the top anchor support is determined by summing moments about that support. Methodologies used to determine moments below the upper anchor can be as illustrated for granular soil one-tier and multitier systems. The total load determined by the EPFs or the Terzaghi and Peck soft to medium clay diagram has a safety factor on the shear strength of about 1.3 (Long, Weatherby, and Cording 1998). 
Earth pressures for soft to medium clay correspond to a state of limiting equilibrium. Therefore, limiting equilibrium analyses can be used to calculate the total lateral load that should be distributed to a flexible wall system using the apparent pressure diagram in Figure 5.6. A safety factor of 1.3 is commonly used for wall systems that deflect sufficiently to approach active state earth pressure conditions in the retained soil. Higher factors of safety are used for those walls that have little outward movement and are therefore closer to an at-rest pressure state rather than an active pressure state.

\subsubsection{Apparent earth pressure diagrams for soft to medium clays in soils subject to deep-seated failure}

Permanent ground anchor walls are generally not recommended for sites where the bottom of the wall is underlain by deep deposits of weak soils. However, temporary earth-retaining tieback walls are sometimes used at these locations. Tieback walls constructed at soft to medium soil sites can experience excessive deflections. Clough and O'Rourke (1990) indicate that excavations in soft to medium clays can result in large vertical and horizontal movements so that there is a chance for severe to very severe damage to structures adjacent to the excavation.

Earth pressures computed based on the soft to medium clay diagram of Figure 5.6 underestimate the total lateral earth load when weak soils extend below the bottom of the wall. Loads higher than those predicted develop when the soil below the wall yields plastically. Terzaghi, Peck, and Mesri (1996) recommended that Henkel's method be used to calculate a value of $K_{a}$ to be used to determine the total load with the soft to medium clay apparent earth pressure diagram providing the basis for the total earth pressure load determination. The total load for soft to medium clays is equal to $0.875 K_{A} \gamma H^{2}$. The distribution of the total load is in accordance with Figure 5.6. The earth pressure coefficient $\left(K_{A}\right)$ for soft to medium clays, developed by Henkel (1971), includes the consideration of deep-seated failures in soft to medium clays below the cut as shown in Figure 5.7.

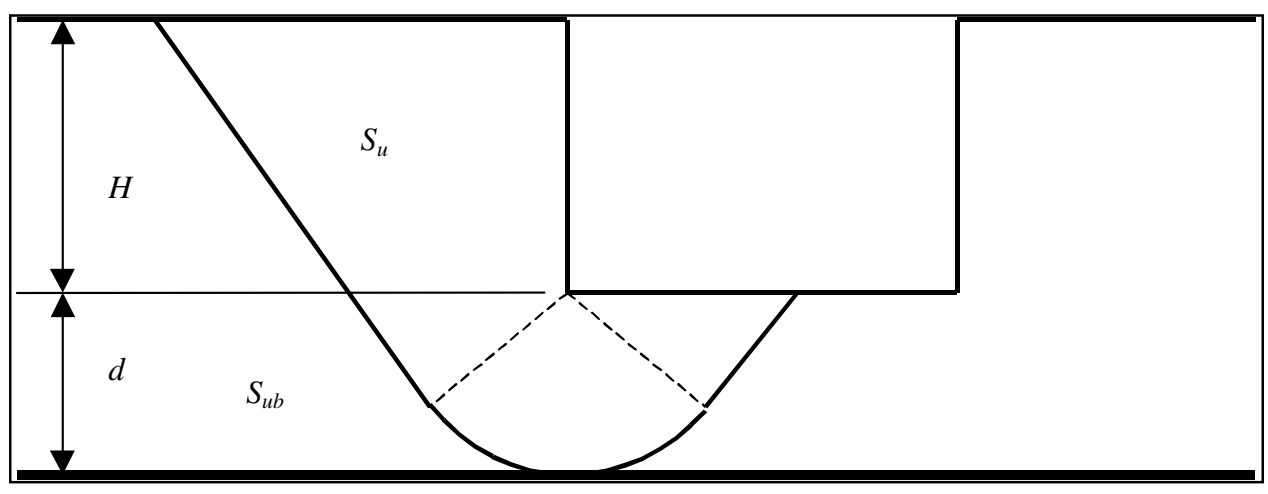

Figure 5.7. Failure surface assumed for Henkel's method (adapted from Figure 22; Sabatini, Pass, and Bachus 1999) 
According to Henkel,

$$
\begin{aligned}
K_{A}= & 1-\frac{4 S_{u}}{\gamma H}+2 \sqrt{2} \frac{d}{H}\left[1-(2+\pi) \frac{S_{u b}}{\gamma H}\right] \\
& =1-\frac{4 S_{u}}{\gamma H}+2 \sqrt{2} \frac{d}{H}\left(1-\frac{5.14 S_{u b}}{\gamma H}\right)
\end{aligned}
$$

(non-SI units)

(SI units)

where

$S_{u}=$ undrained shear strength of the soil through which the excavation extends

$d=$ depth of failure surface below the cut

$S_{u b}=$ strength of the soil providing bearing resistance

Limiting equilibrium analyses are generally recommended for determining the total earth load on temporary tieback walls constructed in soft to medium clays and subject to deep-seated failures. Limiting equilibrium methods can account for plastic yielding, basal heave, and failure mechanisms analyzed by Henkel.

\subsubsection{Total load by sliding wedge analysis}

As described in Sabatini, Pass, and Bachus (1999), a sliding wedge force equilibrium method may be used to evaluate the total horizontal load required to provide stability to a vertical cut. An example failure surface, free body diagram, and force vector diagram are shown in Figure 5.8 for a wall of height $H$ with a soil behind and in front of the wall characterized by an effective stress friction angle $\left(\phi^{\prime}\right)$. It is assumed that the critical potential failure surface passes in front of the anchor bond zone such that the full anchor loads contribute to wall stability. The shear strength is factored by the target factor of safety such that $\phi_{m o b}^{\prime}=\tan ^{-1}\left(\tan \phi^{\prime} / F S\right)$. Mobilized passive resistance is assumed to develop over the wall embedment depth $(d)$. For the assumed failure surface, an interface friction angle $(\delta)$ equal to $\phi_{\text {mob }}^{\prime}$ may be used to calculate the passive earth pressure coefficient.

In the analysis, $P_{R E Q}$ represents the external horizontal force required to provide stability to the vertical cut. This force represents the combined resistance provided by the horizontal component of the anchor forced $(T \cos i)$ and the lateral resistance provided by the embedded portion of the wall $\left(S P_{H}\right)$. The assumption that $\left(P_{R E Q}\right)$ is horizontal implies that the vertical resistance provided by the soldier beam $\left(S P_{V}\right)$ is equal in magnitude and opposite in sign to the vertical component of the ground anchor loads $(T \sin i)$. This assumption should be verified using the procedures described in Chapter 8 to evaluate axial capacity of the wall. The required force $\left(P_{R E Q}\right)$ is then calculated as 


$$
P_{R E Q}=\frac{1}{2} \gamma H^{2}\left[\frac{(1+\xi)^{2}}{\tan (\alpha)}-K_{p} \xi^{2}\left(\sin (\delta)+\frac{\cos (\delta)}{\tan (\alpha-\phi)}\right)\right] \tan (\alpha-\phi)
$$

where all the terms are defined in Figure 5.8. The solution is found iteratively by adjusting the angle of the potential failure surface $(\alpha)$ and the wall embedment $(d)$ until the greatest $\left(P_{R E Q}\right)$ is found. The value for $K_{p}$ in Equation 5.2 is based on the assumption that the failure surface beneath the bottom of the cut on the passive portion of the soil has a log spiral shape. The passive coefficient, $K_{p}$, can be obtained for a log spiral solution using information provided in NAVFAC (1982) and repeated in Long, Weatherby, and Cording (1998), or can be obtained from Figures 3.11 and 3.12 of Ebeling and Morrison (1992). This load $\left(P_{R E Q}\right)$ should then be redistributed into an apparent pressure envelope for calculating ground anchor loads and bending moments in the exposed portion of the wall. Detailed discussion on the use of this simplified method is provided in Long, Weatherby, and Cording (1998).

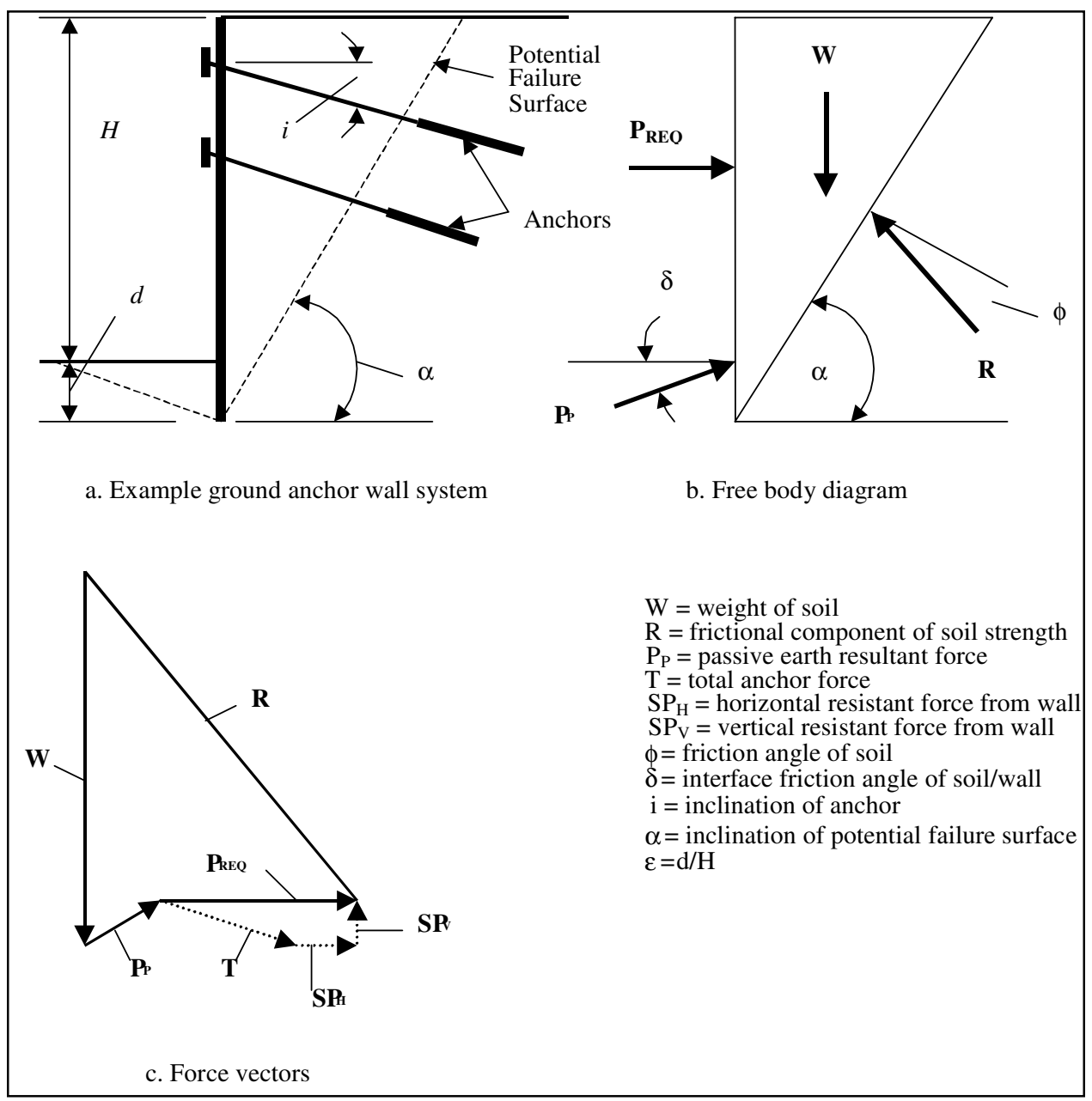

Figure 5.8. Force equilibrium method for anchored walls (after Long, Weatherby, and Cording 1998) 
Sliding wedge and limit equilibrium methods are described in the following FWHA and Corps reports: ETL 1110-2-256 and EM 1110-1-2908 (HQDA); Long, Weatherby, and Cording (1998); Nicholson (1983); Sabatini, Pass, and Bachus (1999); and Weatherby (1998).

\subsubsection{Total load approach for stiff wall systems}

5.3.9.1 General. For the most part, the total load approach as presented above applies to yielding soil conditions where the total load used for constructing apparent pressure diagrams is determined by applying a factor of safety to the soil shear strength parameters. The factor of safety is generally 1.3 when it is expected that partial yielding of the soil retained by the wall (i.e., earth pressures between at-rest and active) will occur. This would be the case for the construction of typical tieback walls where some wall movement can be tolerated. Factors of safety higher than 1.3 are often used when structures sensitive to settlement are founded adjacent to the excavation. In certain circumstances, because the wall is stiff rather than flexible, active pressure conditions may not develop in the soil. The wall may be stiff because wall section properties must be increased to carry hydrostatic loads, or because they must be increased to meet displacement performance objectives. In certain circumstances, the toe of the wall may be embedded in rock, restricting the ability of the wall toe to move toward the excavation as normally occurs with typical top-down construction sequencing. In such cases it may be difficult for "near" active conditions to develop in the soil. In addition, the actual pressure distribution may be closer to triangular than to the trapezoidal pressure distribution assumed for typical top-down construction. The usual assumption is to assume triangular distribution of soil pressure when evaluating bending moments in stiff tieback wall systems. An evaluation is generally performed for all stages of construction.

5.3.9.2 Total load for at-rest pressure conditions. Under circumstances where the wall will not displace sufficiently for "near" active pressure states (with factor of safety equal to 1.3) to occur, the wall can be designed assuming at-rest pressure conditions. In the context of anchored wall design using steel soldier beams or sheet-pile wall elements, design earth pressures based on at-rest conditions are not typically used. Using at-rest earth pressures implicitly assumes that the wall system undergoes no lateral deformation. This condition, however, may be appropriate for use in designing heavily preloaded, stiff wall systems (Sabatini, Pass, and Bachus 1999). It should be recognized that, contrary to the above statements, Teng recommended using at-rest pressure conditions for the design of sheet-pile walls anchored with prestressed tiebacks (U.S. Steel 1969, pp 62-63). Teng suggested using the following $K_{0}$ values:

$$
\begin{aligned}
0.35-0.60 & \text { for sand and gravel } \\
0.45-0.75 & \text { for clay and silt } \\
\geq 1.00 & \text { for overconsolidated clays }
\end{aligned}
$$

For homogeneous soils, the total load assuming at-rest conditions would be 


$$
K_{0} \gamma \frac{H^{2}}{2}
$$

When the total load is determined by limiting equilibrium analyses, the total load or external horizontal force required to provide stability to the vertical cut $\left(P_{R E Q}\right)$ can be determined by applying a factor of safety to the soil shear strength. In this method, available soil strength parameters are reduced by a factor of safety equal to about 1.5 and then used in limiting equilibrium analysis to obtain the total load $\left(P_{R E Q}\right)$ representing at-rest pressure conditions. The reduced soil parameters are defined as the mobilized strength

where

$$
\phi_{m o b}=\tan ^{-1}\left(\frac{\tan \phi}{S F}\right)
$$

and

$$
c_{m o b}=\frac{c}{S F}
$$

and the values $\phi$ and $\mathrm{c}$ represent the available friction angle and available cohesive strength.

5.3.9.3 Pressure distribution for stiff wall systems. When the tieback wall system is stiff, or when the tieback wall toe is embedded in rock or stiff soils that severely restrict toe movement during top-down excavation, the pressure distribution may be closer to triangular than to trapezoidal. It has been suggested that, for stiff diaphragm walls, staged excavation analysis be performed using triangular earth pressure distribution (Ratay 1996). Sometimes, an apparent pressure diagram enveloping both triangular and trapezoidal pressure distributions is used to determine anchor loads. This approach, illustrated in Figure 5.9, has been used to design the tieback anchors for the Bonneville Navigation Lock guard wall. It can be used assuming either active (with factor of safety equal to 1.0) or at-rest pressure conditions with respect to the triangular pressure segment of the earth pressure diagram. However, if used with active pressure conditions, it should be demonstrated that an active state can develop behind the wall even though little movement is occurring at the toe. The Bonneville guard wall anchors were designed assuming at-rest pressure conditions. For the stiffer wall systems it is common practice to assume a triangular distribution of soil pressure and evaluate each stage of construction. In this type of analysis, the vertical element resisting horizontal soil pressures (soldier beam, secant pile, or reinforced concrete wall) is treated as a continuous beam spanning between anchor locations, or anchor location and point of ground fixity in the case of the lowermost span. In some cases the system is analyzed as a continuous beam on nonrigid supports where the displacement occurring at a tieback anchor location (before the tieback is installed) is applied as a specified support displacement. Staged analysis for stiff wall systems is described below and in Ratay (1996). 


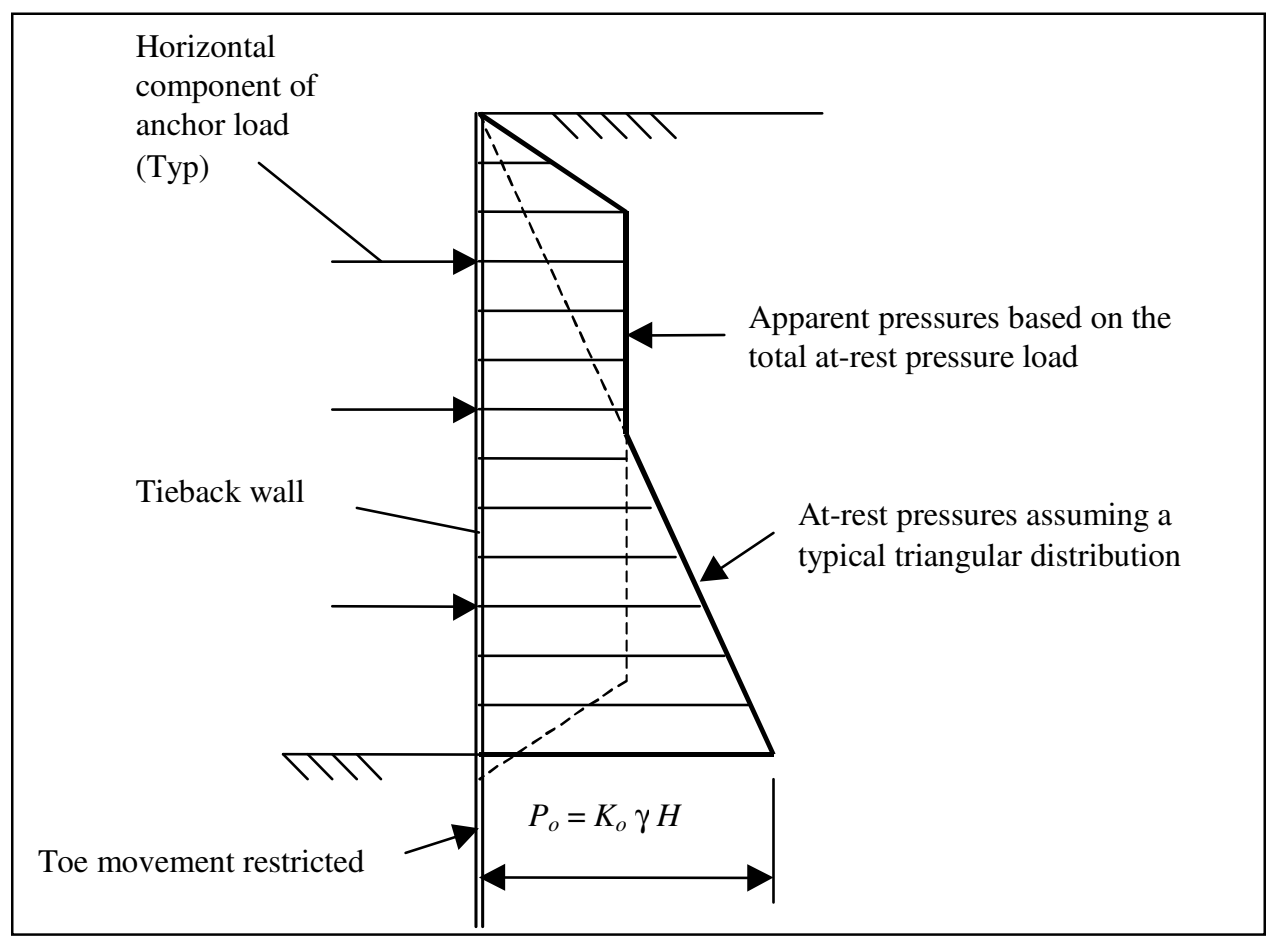

Figure 5.9. Pressure distribution when toe movements are restricted

5.3.9.4 Staged analysis for stiff wall systems. Stiffer wall systems are generally analyzed for conditions that exist before each level of tieback anchors is installed, as well as for the final condition where all tiebacks have been installed and the excavation is to final grade. A beam on rigid supports (RIGID) analysis is generally used. However, continuous beam on nonrigid support and beam on elastic foundation (Winkler) analyses are also commonplace. The latter analysis is described in Chapter 6.

\subsection{Continuous beam on rigid supports analysis. The salient} features of a RIGID analysis have been described above and in Kerr and Tamaro (1990). When used in a staged excavation analysis, assumptions similar to those made in the design of cantilever and anchored sheet-pile walls (U.S. Steel 1969) are used to estimate earth pressures on each side of the wall, and to determine the below-grade point (fictitious support location) satisfying force and moment equilibrium. Driving side pressures can be near at-rest or at rest when high tieback prestress loads restrict wall lateral movement (Ratay 1996).

Passive earth pressures are applied to the resisting side of the wall, and net pressures are determined and used in a structural analysis to obtain anchor loads, shears, and bending moments. Since the first-stage excavation analysis is statically determinate, it is possible by simple hand calculation to calculate the maximum moments and shears. In the second stage and subsequent excavation analyses, it is generally assumed that a point of contraflexure coincides with the zero net pressure point. Using this assumption, the upper portion of the anchored tieback wall can be treated as an equivalent beam that is simply supported at anchor locations and at the first point of zero net pressure intensity. The secondstage excavation analysis, as with first stage, is statically determinate and 
therefore the maximum wall moments and shears can be determined by simple hand calculations. Subsequent excavation stages are generally evaluated using the same equivalent beam approach used for the second-stage excavation. These analyses, however, will involve a beam on three or more supports and are therefore statically indeterminate. The continuous beam on rigid supports analysis is demonstrated in Strom and Ebeling (2002a).

Full passive resistance has been used as resisting side earth pressure in the staged excavation analysis of stiff wall systems (i.e., Bonneville guard wall). However, mobilized passive earth pressure can also be based on the soil shear strength factored by a target factor of safety as described in Section 5.3.8. There is no universal agreement in the engineering community as to whether full or mobilized passive earth pressure should be used with respect to the staged excavation analysis of stiff wall systems. This subject requires further research. When a RIGID analysis is used to evaluate each stage of excavation, the process is similar to that illustrated in Figure 5.10.

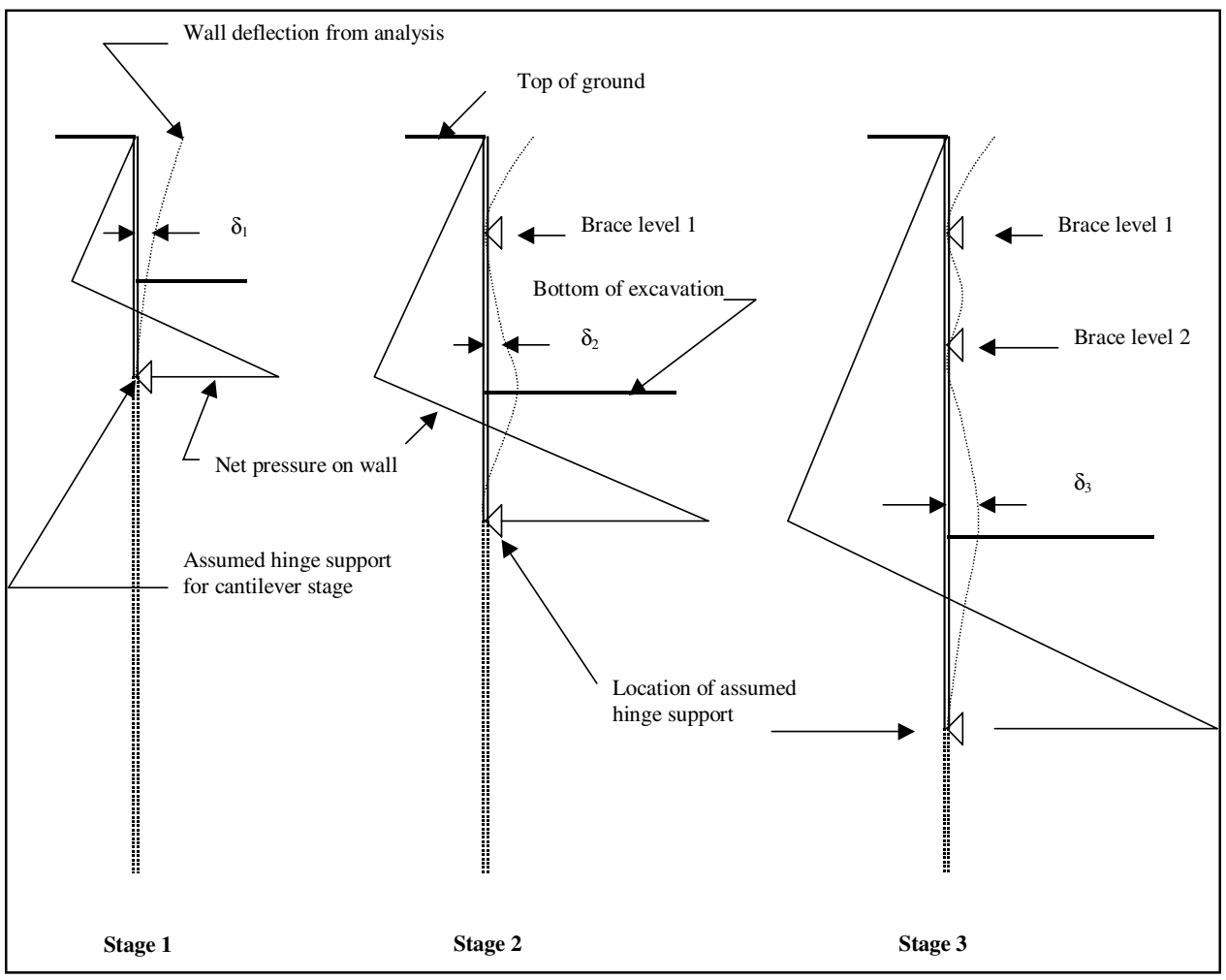

Figure 5.10. Modeling for staged excavation analysis by RIGID method

In general, the continuous beam on rigid supports staged excavation analysis will provide reasonable preliminary estimates of anchor forces and wall bending moments. However, this type of construction sequencing analysis is often followed by a beam on elastic foundation (Winkler) construction sequencing analysis to verify anchor forces (Pfister, Evers, Guillaud, and Davidson 1982).

5.3.9.4.2 Continuous beam on nonrigid supports analysis. In the aforementioned RIGID analysis, tieback anchor locations are treated as rigid 
supports with zero lateral displacements for all stages of excavation. Two approaches are used in a continuous beam on nonrigid supports analysis. In the approach described in Kerr and Tamaro (1990), the lateral displacement occurring before the tieback is installed is applied to the support (anchor location) and kept at that specified value throughout the remaining stages of the analysis. This type of analysis can be easily accomplished with most structural analysis software. The results are considered to be more realistic than those provided by the RIGID analysis (Kerr and Tamaro 1990). In the continuous beam on nonrigid supports analysis described in Ratay (1996), the tiebacks are modeled as axial loaded elastic members. To account for the wall deflection that takes place at the level of the next lower tieback before it is installed, an initial support displacement is applied at the fixed end of that next tieback. This initial displacement $\delta$ is equal to the wall deflection $\delta$ computed at that level immediately prior to installation of the tieback. The continuous beam on nonrigid support analysis approach described in Ratay (1996) is illustrated in Figure 5.11. It should be remembered, however, that the tiebacks are prestressed (preloaded) and that any displacement contribution due to elastic lengthening of the tieback will only be for that increment of load above the anchor preload. Assuming this displacement contribution is negligible, the method described in Ratay (1996) is identical to that described in Kerr and Tamaro (1990).

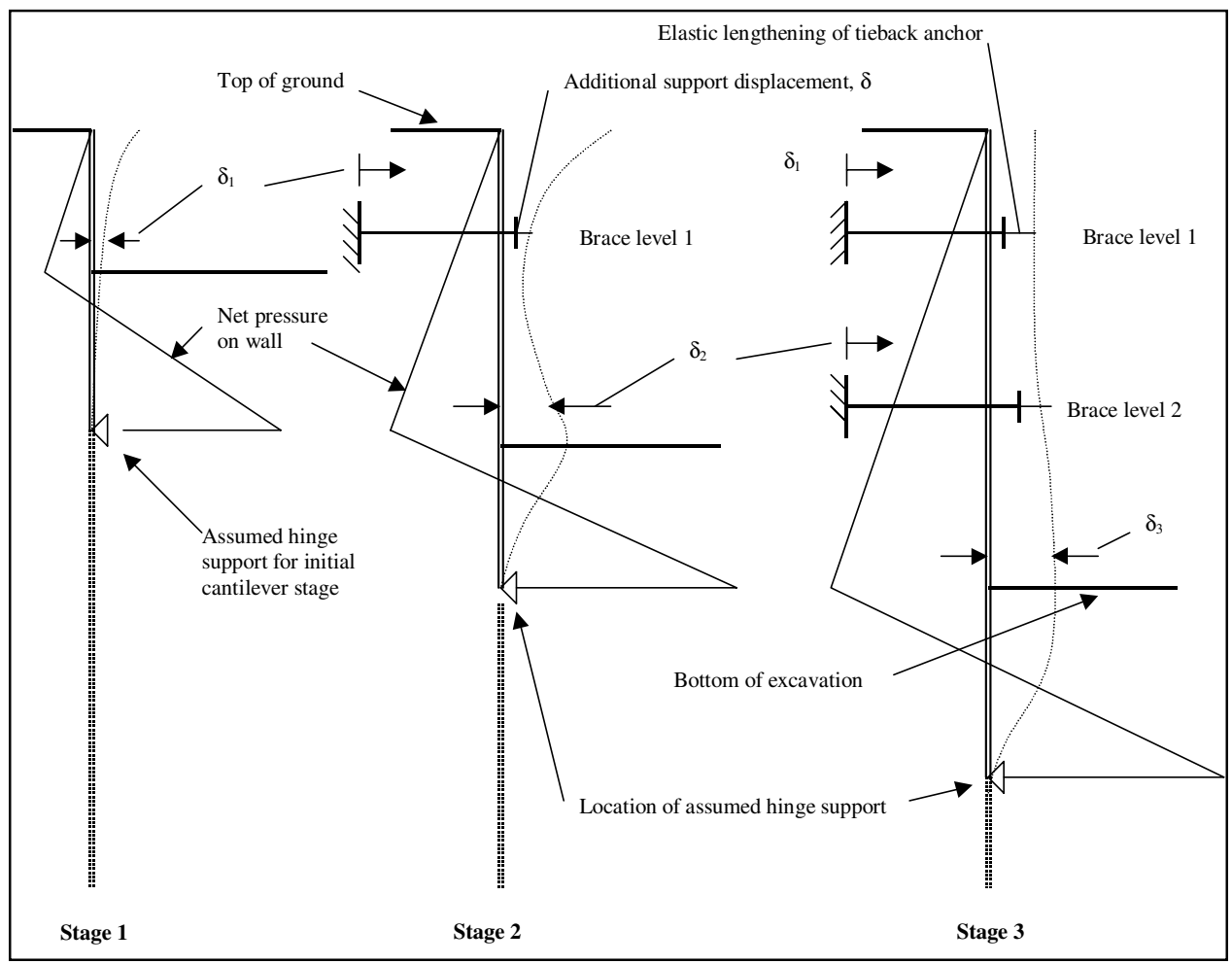

Figure 5.11. Modeling for staged excavation analysis by nonrigid support method 


\subsection{Surcharge Loads}

\subsubsection{General}

Loads due to stockpiled material, roadways, railways, and other influences resting on the soil in the vicinity of the tieback wall increase lateral pressures on the wall. When a wedge method is used for calculating the earth pressures, the resultant of the surcharge acting on the top surface of the failure wedge is included in the equilibrium of the wedge. If the soil admits to application of the coefficient method, the effects of surcharges (other than a uniform surcharge) are evaluated from the theory of elastic solutions presented in the following paragraphs.

\subsubsection{Uniform surcharge}

Where uniform surcharges $\left(p_{s}\right)$ (see Figure 5.12) are present, the vertical effective stress increases by the amount of the surcharge, and the horizontal earth pressure due to the surcharge is assumed to be a uniform increase in lateral stress over the entire height of the wall equal to

$\begin{aligned} K_{A}\left(p_{s}\right) & \text { for yielding soil conditions } \\ \text { and } K_{0}\left(p_{s}\right) & \text { for nonyielding soil conditions }\end{aligned}$

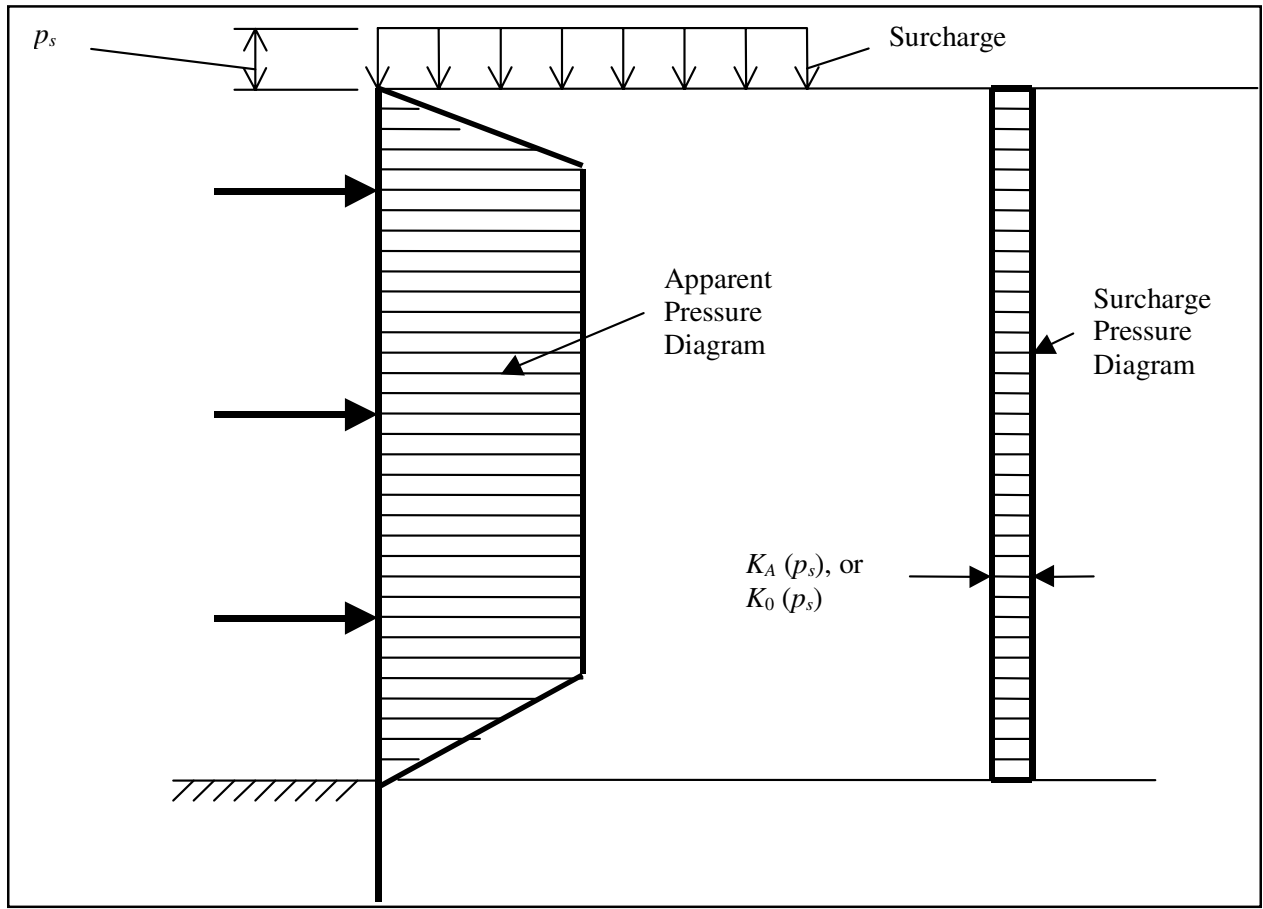

Figure 5.12. Apparent earth pressure diagram - with uniform surcharge 


\subsubsection{Point loads, line loads, and strip loads}

Point loads, line loads, and strip loads are vertical surface loadings that are applied over limited areas as compared to uniform surcharge loads. As a result, the increase in lateral earth pressure used for wall system design is not constant with depth, as is the case for uniform surcharge loads. These loadings are typically calculated using equations based on elasticity theory for lateral stress distribution with depth. Lateral pressures resulting from these surcharges should be added explicitly to the design (apparent pressure) earth pressure envelope.

5.4.3.1 Point loads. A surcharge load distributed over a small area may be treated as a point load. The equations for evaluating lateral pressures due to point loads are given in Figure 5.13. Because the pressures vary horizontally parallel to the wall, it will be necessary to determine the point load horizontal pressure tributary to a particular soldier beam (or tributary to a line of vertical anchors) and apply this pressure in accordance with the vertical distribution pattern shown in Figure 5.13.

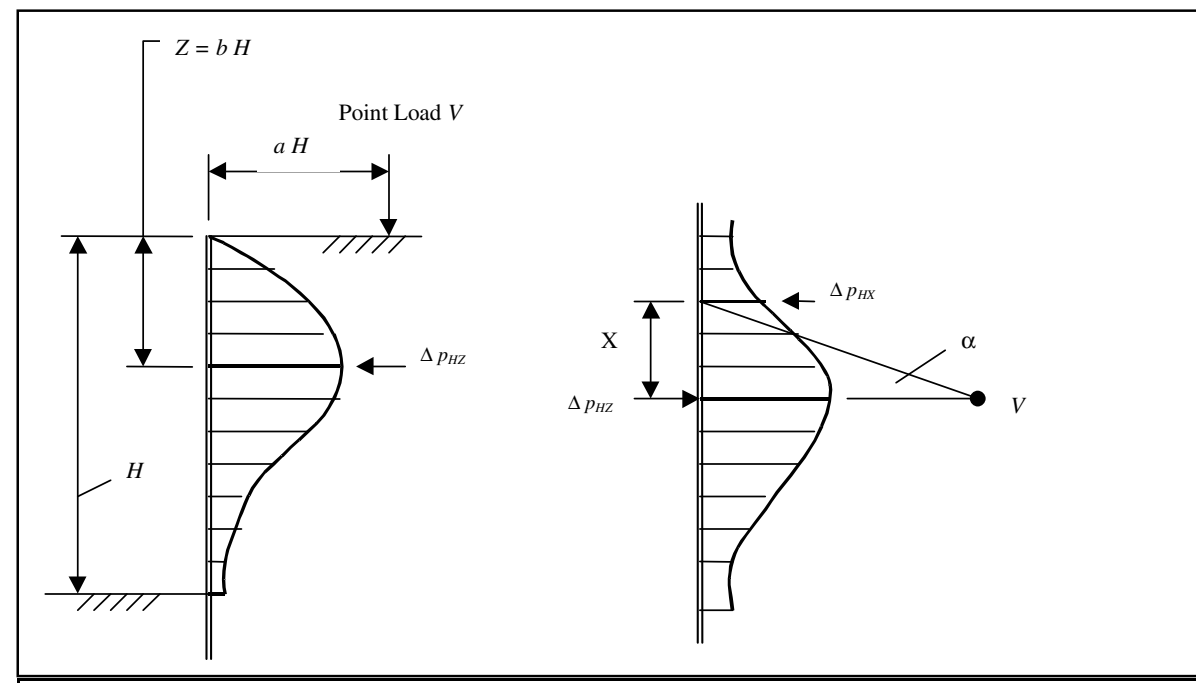

Increase in horizontal pressure, $\Delta \mathrm{p}_{\mathrm{HZ}}$, on a section through point load $V$.

$$
\begin{array}{ll}
\Delta p_{H Z}=\frac{V}{H^{2}}\left(\frac{a^{2} b^{2}}{\left(a^{2}+b^{2}\right)^{3}}\right) & \text { For } \mathrm{a}>0.4 \\
\Delta p_{H Z}=\frac{0.28 V}{H^{2}}\left(\frac{b^{2}}{\left(0.16+b^{2}\right)^{3}}\right) & \text { For } \mathrm{a} \leq 0.4
\end{array}
$$

Increase in horizontal pressure, $\Delta \mathrm{p}_{\mathrm{HX}}$, at distance $\mathrm{X}$ from plane of load $V$.

$$
\begin{aligned}
& \alpha=\tan ^{-1}\left(\frac{X}{a H}\right) \\
& \Delta p_{H X}=\Delta p_{H Z} \cos ^{2}(1.1 \alpha)
\end{aligned}
$$

Figure 5.13. Increase in pressure due to point load (from EM 1110-2-2502) 
5.4.3.2 Line loads. A continuous concentrated load parallel to the longitudinal axis of the wall is considered a line load. The lateral earth pressure due to a line load can be determined using the equations in Figure 5.14.

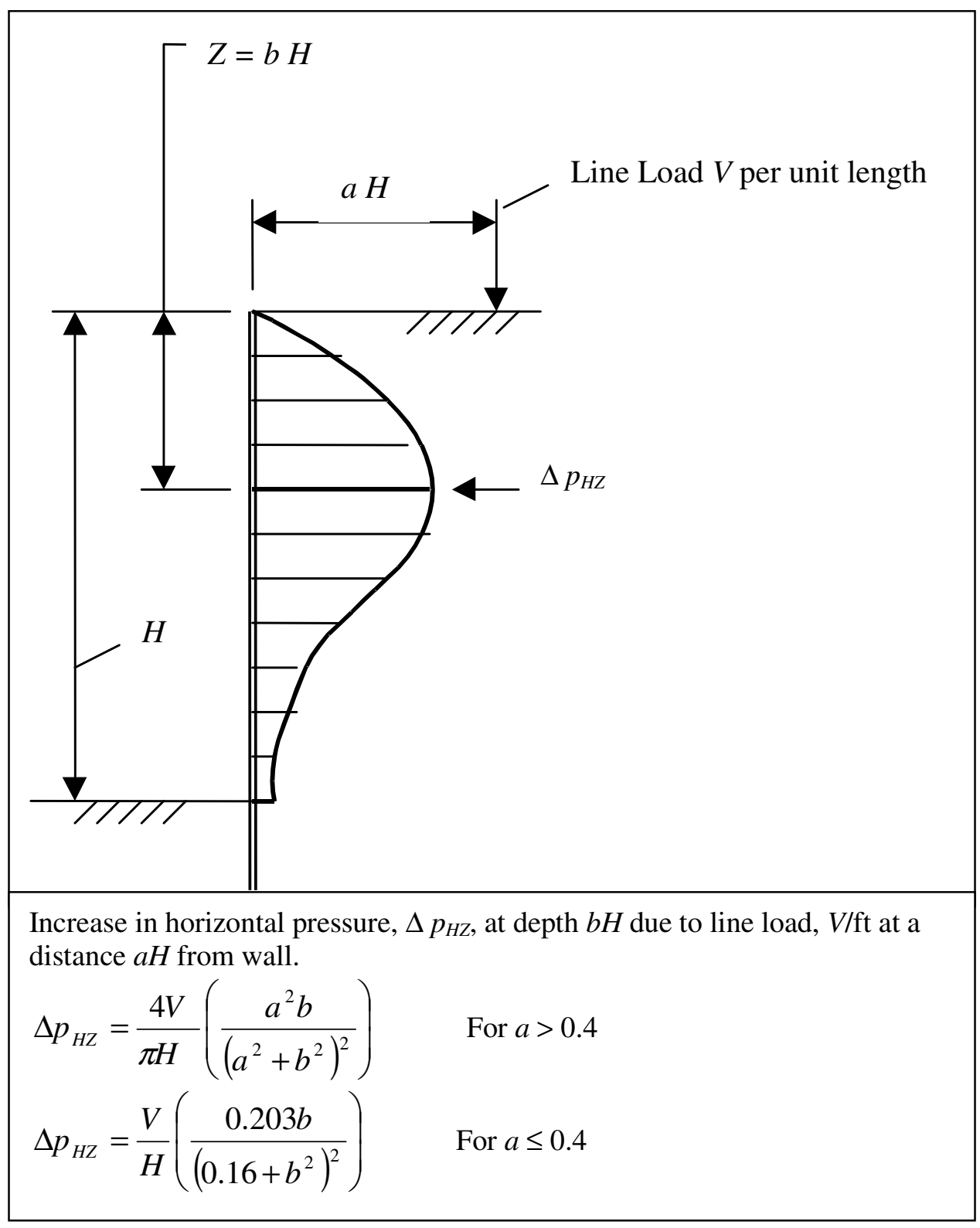

Figure 5.14. Increase in pressure due to line load (from EM 1110-2-2502)

5.4.3.3 Strip loads. A continuous uniform load of finite extent parallel to the longitudinal axis of the wall is considered a strip load. The lateral earth pressure due to a strip load can be determined using the equations in Figure 5.15. 


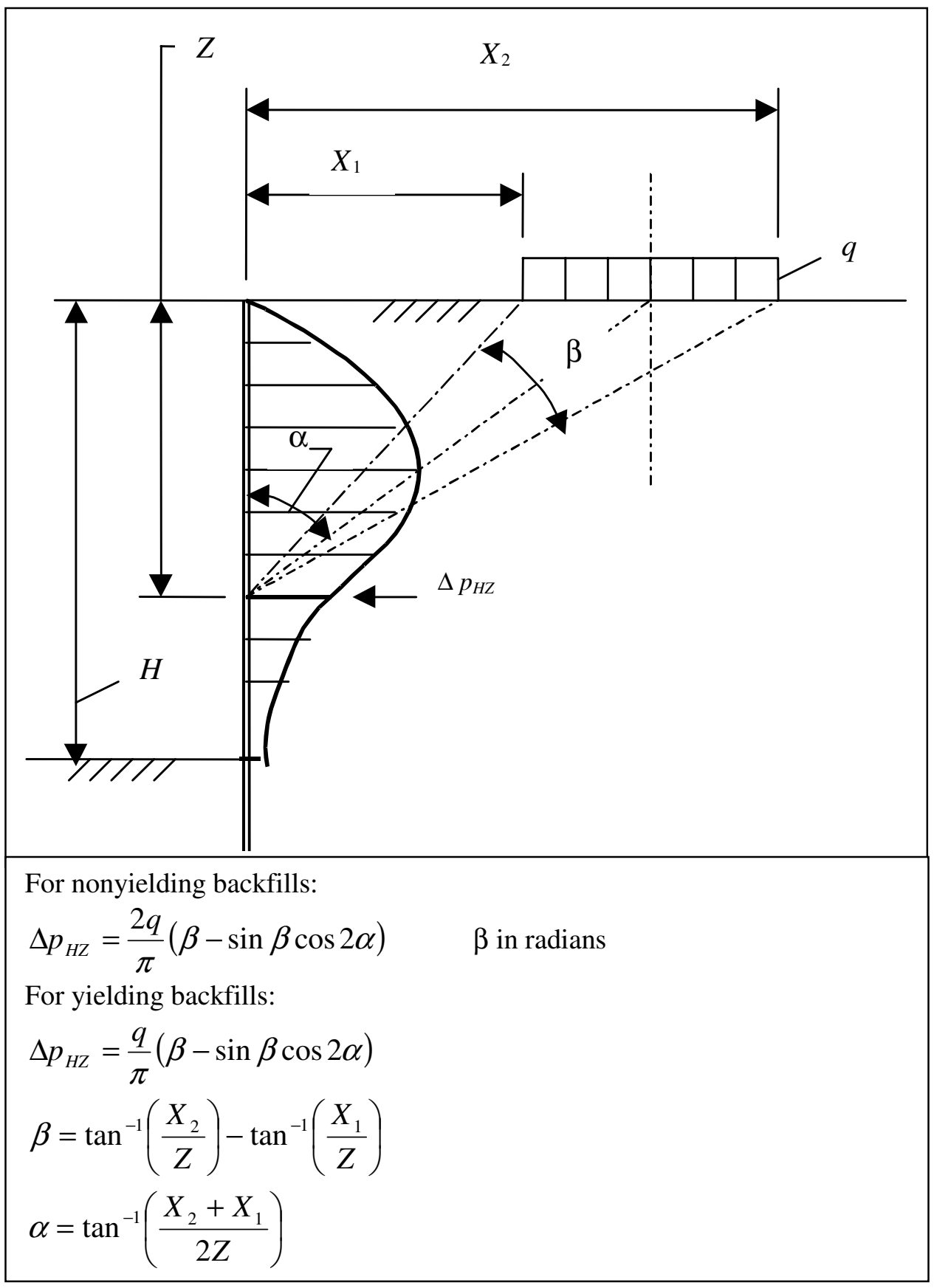

Figure 5.15. Increase in pressure due to strip load (from EM 1110-2-2502) 


\subsection{Water in the Retained Soil}

\subsubsection{General}

Water loads must be considered for excavations involving relatively impermeable walls, and for permanent walls that are subject to fluctuating water levels. There is no universal agreement in the engineering community as to how to account for a water table in the retained earth of a tieback wall system. This is a subject that requires further research. To date, two approaches are available for combining earth and water pressures: the average effective unit weight approach and the total unit weight approach. Both should be considered in the design of tieback wall systems subjected to hydrostatic pressures. Sands and other freedraining soils are designed for both the short term and long term (postconstruction/permanent) by the average effective unit weight approach (i.e., effective stress analysis) using effective weights (buoyant unit weights) and pore-water pressures. For short-term loadings, clay soils are evaluated based on a total unit weight approach (i.e., total stress analysis) using saturated soil weights for submerged soils and moist unit weights for soils above the water table. Internal pore pressures within the submerged soil mass are not considered explicitly in total stress analyses, but the effects of the pore pressures for the undrained condition are reflected in the soil's undrained shear strength. For long-term loadings, clay soils are generally evaluated using the average effective unit weight approach in the same manner as for free-draining soils according to Weatherby (1998).

\subsubsection{Combining earth and water pressures}

In a majority of retaining structures, the lateral pressures are caused by a combination of earth and water pressures. A hydrostatic water table within the retained earth is a common assumption. The validity of this assumption and the simplified calculation procedures that follow are to be carefully evaluated by the design team. In the case of earth submerged in water, the following two assumptions are sometimes used:

a. The lateral pressure of earth and water for hydrostatic water table conditions is taken as the full water pressure, plus the earth pressure based on the buoyant weight of the material (saturated weight minus the unit weight of water). This assumption is used for sand and other freedraining soils for both short- and long-term loadings and is used for clays for long-term loadings only. For cases other than hydrostatic (i.e., steadystate seepage conditions), the resultant body force used in the effective lateral earth pressure computations may be obtained by combining the buoyant soil weight and the seepage force. See Equation 27 (Ebeling and Morrison 1992; p 33) for computation of the effective unit weight to be used in computation of the effective (vertical) weight of the backfill.

$b$. The total pressure of earth and water is taken as the pressure of the earth only, where the weight of the earth per cubic foot is equal to the weight of a cubic foot of earth saturated with water. This assumption is used for clays for short-term loading (undrained) conditions. 
External water pressures, if present (i.e., in front of the retained multianchored wall after the excavated site is flooded), should be taken into account in the analyses, whether they are performed in terms of total or effective stresses.

5.5.2.1 Average effective unit weight approach-Assumption $a$. The above conclusion has led to an average effective unit weight approach for use in the design of braced cofferdams and tieback walls. In this approach, an average effective unit weight is determined by either of two methods:

Method 1 The average effective weight $\left(\gamma_{e}\right)$ is determined by multiplying submerged height of wall $\left(H_{1}\right)$ by the buoyant weight $\left(\gamma_{1}\right)$, multiplying the nonsubmerged height of wall $\left(H_{2}\right)$ by the moist weight $\left(\gamma_{2}\right)$, adding the two quantities, and dividing by the total height of wall $(H)$, or

$$
\gamma_{e}=\frac{\gamma_{1} H_{1}+\gamma_{2} H_{2}}{H}
$$

An apparent pressure diagram is developed based on the average effective weight $\left(\gamma_{\mathrm{e}}\right)$, and the water pressure is then added separately to obtain the total pressure to be used to design the wall and anchorages. This method assumes a hydrostatic water table in the retained soil. The Method 1 approach with respect to the design of braced cuts in sand is illustrated in Figure 1 of U.S. Steel (Dec 1969, p 57, Sheet Piling Design Manual).

Method 2 Method 2 follows procedures presented in Ebeling and Morrison (1992). In this method, instead of a weighting process based on the heights of wall tributary to submerged and nonsubmerged soils, weighting is based on the submerged and nonsubmerged soil areas contained in the failure wedge. Referring to Figure 5-16 (or Figure 4.13 of Ebeling and Morrison 1992):

$\gamma_{e}=\left[\frac{A_{1}}{A_{1}+A_{2}}\right] \gamma_{1}+\left[1-\frac{A_{1}}{A_{1}+A_{2}}\right] \gamma_{2}$

or

$$
\gamma_{e}=\left[\frac{H_{1}}{H}\right]^{2} \gamma_{1}+\left[1-\left(\frac{H_{1}}{H}\right)^{2}\right] \gamma_{2}
$$

where 


$$
\begin{aligned}
& \gamma_{1}=\gamma_{\text {Saturated }}-\gamma_{\text {Water }} \\
& \gamma_{2}=\gamma_{\text {Moist }}
\end{aligned}
$$

Method 2 more appropriately considers the weights of the submerged and nonsubmerged soils in the failure wedge and is therefore preferred over Method 1. When used in limiting equilibrium analyses, Method 2 will provide the correct solution. As with Method 1, these calculations assume a hydrostatic water table in the retained soil.

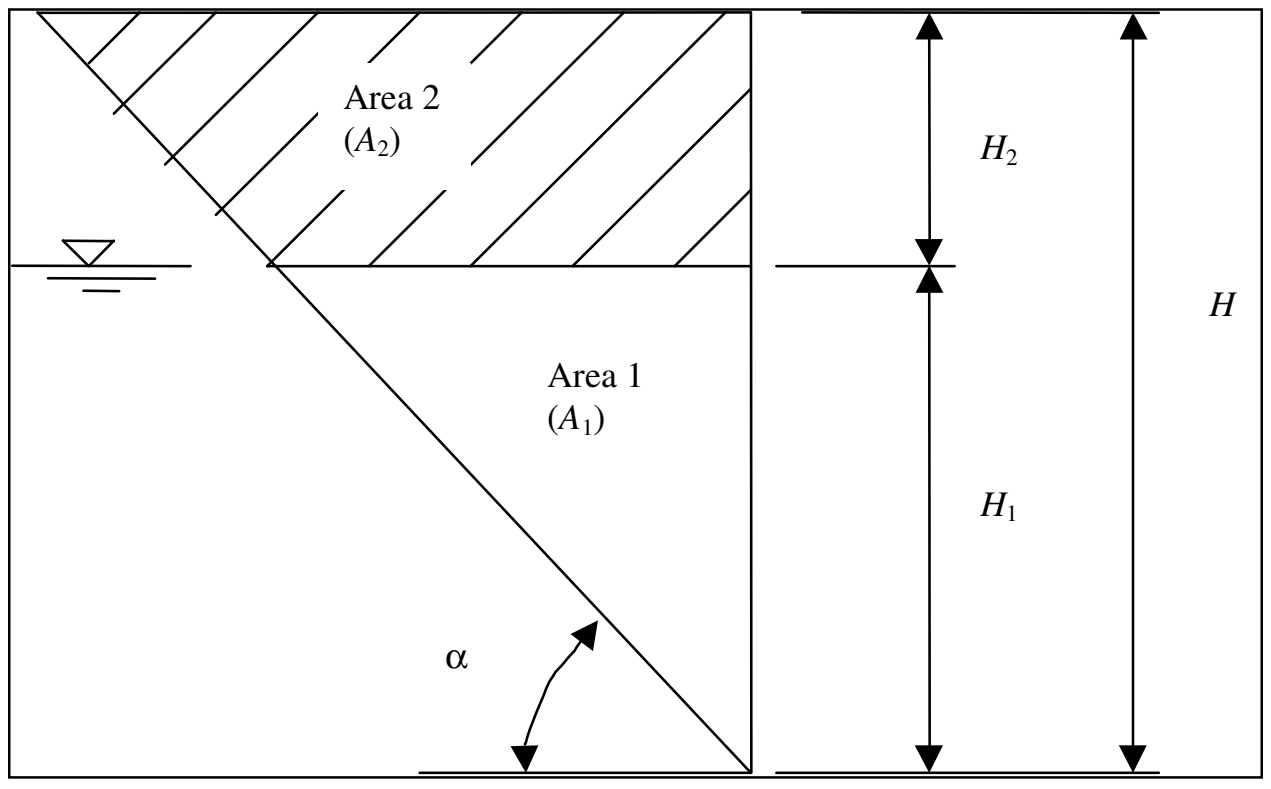

Figure 5.16. Method 2, effective unit weight for partially submerged soils (from Ebeling and Morrison 1992)

The average effective weight approach is illustrated in Figure 5.17. As stated above, this approach is often used to estimate loads on tieback walls that retain sands and other free-draining soils. The average effective weight approach for sands and other free-draining soils is applicable to both long- and short-term conditions. It can also be used to estimate the loads on walls retaining clay soils under long-term (drained) conditions according to Weatherby (1998).

5.5.2.2 Total unit weight approach-Assumption $\boldsymbol{b}$. With respect to the above effective unit weight approach, Liao and Neff (1990) state:

In excavations with relatively impermeable support walls and/or where significant drawdown of the water table does not occur, geotechnical engineers often adapt Peck's recommendations using the buoyant unit weight $(\gamma)$ rather than the total unit weight $(\gamma)$ of the soil (or possibly a pro-rated unit weight in cases where the water table is at an intermediate depth between the ground surface and the excavation bottom). Then the lateral pressure due to hydrostatic is superimposed onto the earth 
pressure diagram. Though this approach follows a logic of sorts, the validity of this method is questioned by engineers who view Peck's recommendations as a purely empirical method based on total stresses. To cast Peck's method into an effective stress framework by using buoyant unit weights, in this view, [would] be equivalent to unnecessarily tampering with a successful method of design. The proponents of this view would advocate using Peck's method with total unit weights, even when the water table behind excavation wall is not perturbed, and not account for the hydrostatic pressures. Generally this results in more severe loading conditions than the approach adapted using buoyant weights.

With respect to the total unit weight approach, Duncan and Buchignani (1975) indicate that internal pore pressures (within the soil mass) are not considered explicitly in total stress analyses, but the effects of the pore pressures in the undrained tests are reflected in the value(s) of $S_{u}$. If the laboratory specimens are representative of the soils in the field, the pore pressures in the laboratory specimens will be the same as the pore pressures in the field at the locations where the total stresses are the same, and the use of total stress strength parameters from undrained (shear) tests therefore properly accounts for pore pressure effects in short-term, undrained (loading) conditions.

Moist soil unit weights are assigned to the soil regime above the water table, and saturated soil unit weights are used below the water table. 


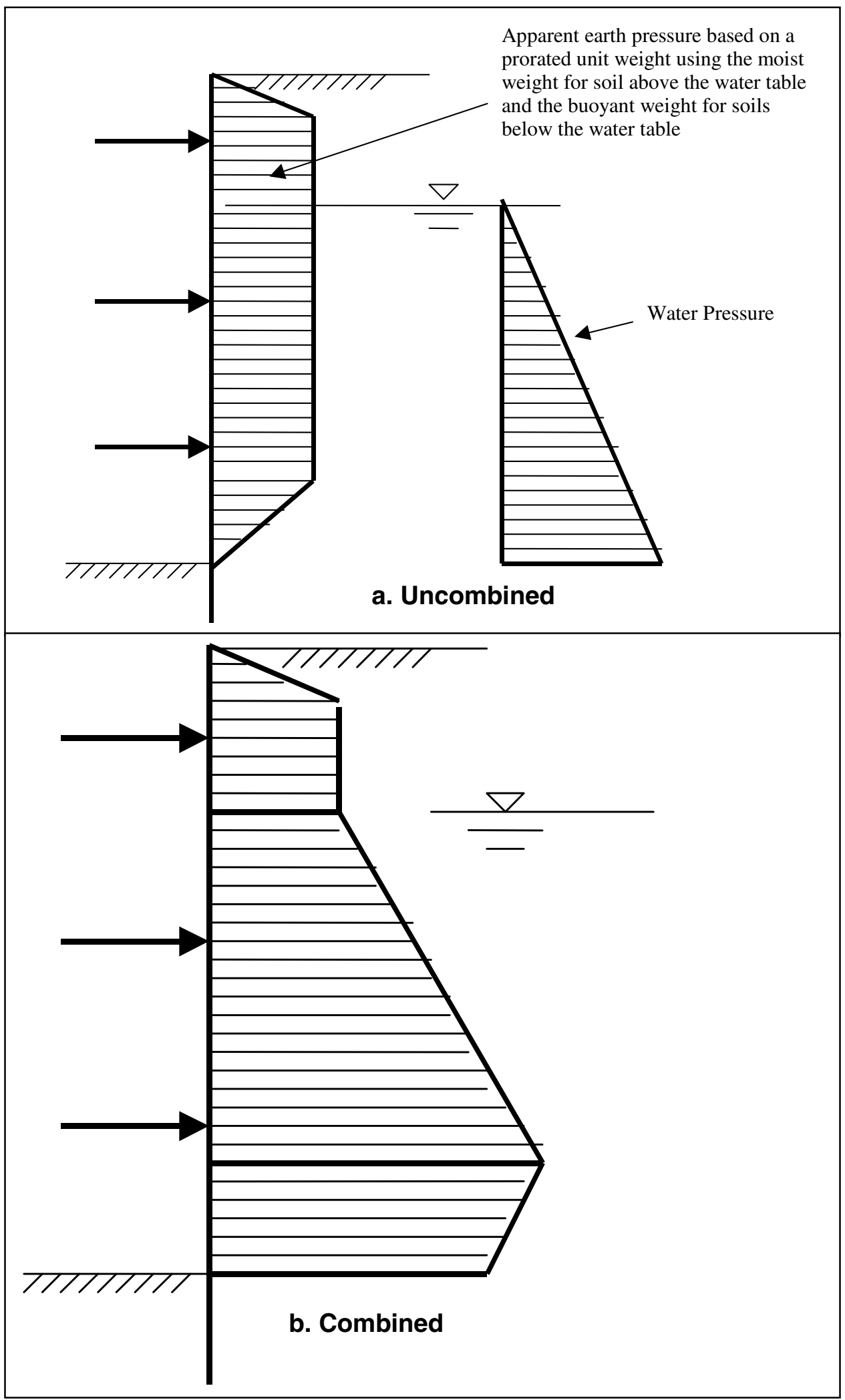

Figure 5.17. Apparent earth pressure diagram computed using effective stress soil strength parameters with hydrostatic water table 


\section{Winkler Analysis Procedure}

\subsection{Introduction}

Beam on elastic foundation analysis, or Winkler spring analysis, is a soilstructure interaction (SSI) method of analysis that enforces compatibility of deflections, soil pressures, and anchor forces while accounting for wall and anchor flexibility. The method is based on a one-dimensional (1-D) finite element representation of the wall/soil system consisting of linearly elastic beamcolumn elements for the wall, distributed nonlinear Winkler springs to represent the soil, and nonlinear preloaded concentrated springs to represent the anchors. In tieback wall design and analysis, the Winkler spring analysis can be used to

- Evaluate the lateral resistance of the tieback wall toe for wall loadings based on apparent pressure distributions.

- Evaluate actual (rather than apparent) soil pressure distributions, wall forces, anchor loads, and displacements at final excavation.

- Simulate in approximate manner construction sequencing, and actual soil pressure distributions, wall forces, anchor loads, and displacements at intermediate stages of construction.

\subsection{Soil Spring Stiffness}

\subsubsection{General}

Design and analysis of pile foundation systems through the use of beam on elastic foundation analysis techniques has been available to engineers for many years. It is natural that engineers would also use this method to obtain the quantities of interest with respect to the design and analysis of tieback wall systems-moments, shears, deflections, anchor forces, and soil pressures. The beam on elastic foundation, or Winkler spring analysis method, considers the behavior of a continuous flexural member with stiffness (EI) supported by infinitely closely spaced soil-springs with stiffness $\left(k_{h}\right)$. Winkler (1867) assumed each spring acted independently (that is, the behavior of one spring has no effect on the adjacent springs). 
In the Winkler analysis, springs can be taken as either linear or nonlinear with their response based on curves that relate soil resistance, $p$, to wall displacement, $y$. In general $p-y$ curves are nonlinear; however, they can be approximated as ideal elastoplastic systems. An idealized elastoplastic representation of $p-y$ response is the basis for the Winkler springs described herein. The $p-y$ curve concept is illustrated in Figure 6.1 with respect to a secant pile tieback wall system. Before excavation begins, the pressures on the secant pile are in equilibrium and therefore the resultant force, $p$, is zero. The secant pile moves toward the excavation as excavation takes place. This movement causes earth pressures on the unexcavated side of the pile to decrease and those on the excavated side to increase, as indicated in Figure 6.1.

A plot of the net pressure difference (reaction), $p$, on the pile versus pile lateral movement, $y$, is designated the $p-y$ curve. These curves can be generated for various elevations along the wall height. These $p-y$ curves are the basis for the idealized elastoplastic springs used in the Winkler analysis. Since for the idealized Winkler spring the relationship between the horizontal reaction $p$ and the displacement $y$ is linear, the ratio $p / y$ is the spring stiffness:

$$
\frac{p}{y}=k_{h}
$$

where the spring stiffness, $k_{h}$, is termed the coefficient of horizontal subgrade reaction (Terzaghi 1955).

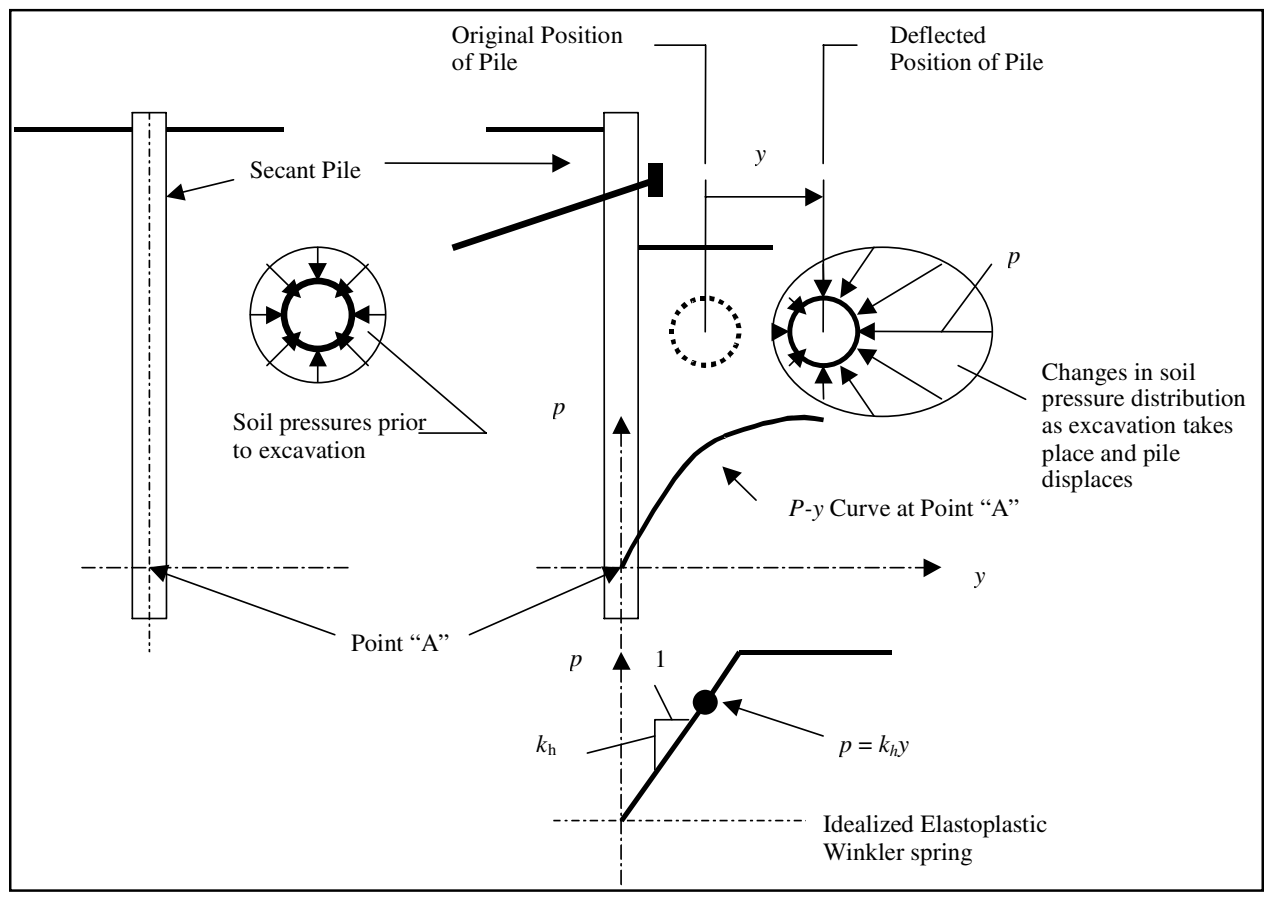

Figure 6.1. Concept of $p-y$ curve 
The Winkler soil spring system for a tieback wall is illustrated in Figure 6.2. These infinitely closely spaced soil springs can have a stiffness that increases linearly with depth to approximate the behavior of cohesionless soils, normally consolidated silts, and normally consolidated clays, or may be constant with depth to represent the approximate behavior of some types of cohesive soils.

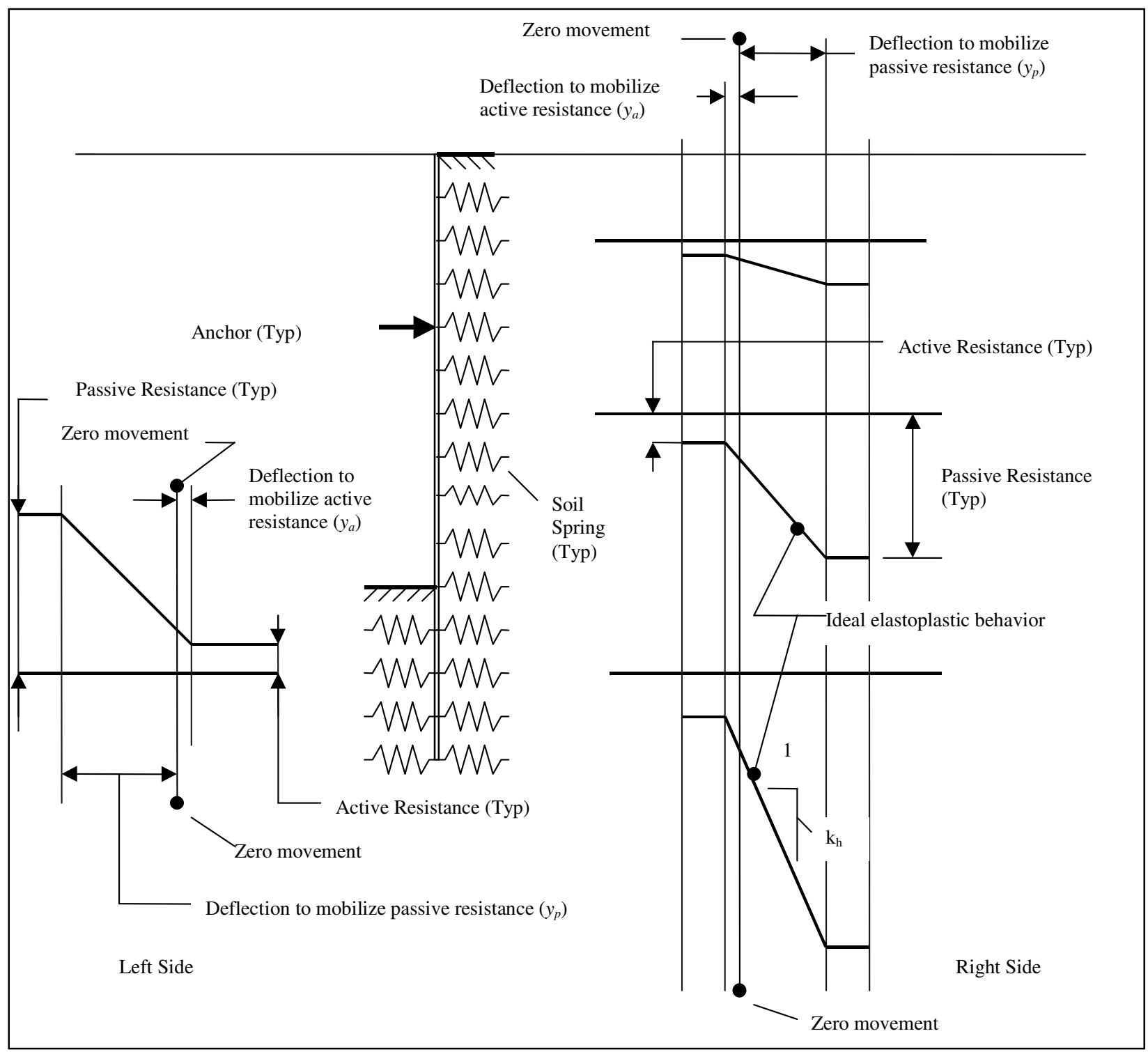

Figure 6.2. Tieback wall soil springs for Winkler analysis

Difficulties in obtaining reasonable results from a Winkler spring analysis often occur because

- The load deformation characteristics of the soil are not linear and may not be suitably represented by ideal elastoplastic behavior.

- The soil stiffness varies with respect to confining pressure and zone of influence. 
- The soil stiffness changes with submergence.

- The ultimate resistance of the soil is dependent on different failure mechanisms depending on whether the soil is near the surface or at some depth below the surface.

- The behavior of discrete wall systems (soldier beam systems) is different from continuous wall systems because the earth pressure distribution behind the wall is different (zone of influence is different) and because soil has a tendency to arch between the structural elements of discrete wall systems.

Simplifying assumptions similar to those made with respect to the behavior of bearing piles and sheet-pile walls subject to lateral loadings are also made with respect to tieback wall systems. These simplifying assumptions are described below.

\subsubsection{Coefficient of horizontal subgrade reaction}

In his treatise on subgrade reaction, Terzaghi (1955) indicates that the coefficient of horizontal subgrade reaction $\left(k_{h}\right)$ is dependent on the deformation characteristics of the subgrade. For stiff (that is, overconsolidated) clays, the deformation characteristics are more or less independent of depth such that the subgrade reaction $(p)$ would be uniformly distributed with respect to depth along the face of the soldier beam or continuous wall, as the case might be. Therefore, for stiff clays, the coefficient of subgrade reaction, $k_{h}$, would be constant with depth. For cohesionless sands, the pressure required to produce a given horizontal displacement increases in direct proportion to the effective confining pressure, which for uniform soil layers would be in direct proportion to the depth (z). Therefore, for cohesionless sands the coefficient of subgrade reaction, $k_{h}$, would increase linearly with depth. The assumption used for sand has also been proven valid for normally consolidated silts and normally consolidated clays (Peck and Davisson 1962). The relationships described above are illustrated in Figure 6.3.

The coefficient of horizontal subgrade reaction, $k_{h}$, is used in a Winkler spring analysis to define the behavior of the soil in the linear elastic range. For cohesionless soils, normally consolidated silts, and normally consolidated clays, the coefficient of horizontal subgrade reaction is a function of the constant of horizontal subgrade reaction, $n_{h}$, for discrete wall systems and is a function of the subgrade constant, $l_{h}$, for continuous wall systems. For stiff clays, the coefficient of horizontal subgrade reaction is a function of $s_{u}$, the undrained unconfined compressive strength. 


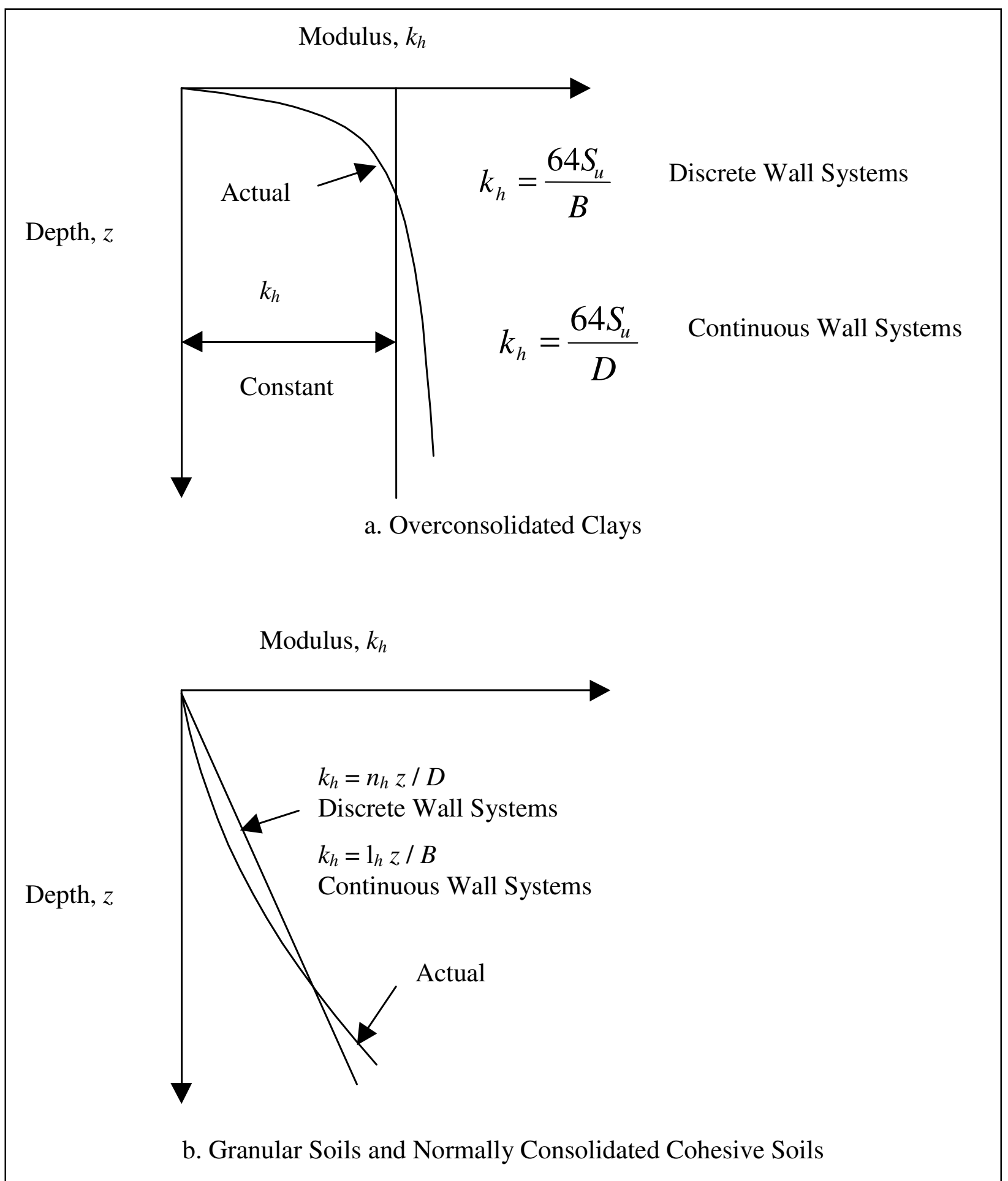

Figure 6.3. Subgrade reaction idealizations 


\subsubsection{Coefficient of horizontal subgrade reaction for discrete wall elements}

This section summarizes the recommendations given in Terzaghi (1955). For soldier beams in stiff clay, the coefficient of horizontal subgrade reaction $\left(k_{h}\right)$ can be assumed to be constant with depth, and taken as equal to

$$
k_{h}=\frac{64 S_{u}}{B}
$$

where

$$
\begin{aligned}
S_{u}= & \text { undrained strength of the soil (equals one half the unconfined } \\
& \text { compressive strength) } \\
B= & \text { soldier beam width }
\end{aligned}
$$

For soldier beams in cohesionless soils, the coefficient of horizontal subgrade reaction $\left(k_{h}\right)$ can be assumed to increase linearly with depth and taken as equal to

$$
k_{h}=n_{h} \frac{z}{B}
$$

\begin{tabular}{|c|c|c|c|}
\hline & & & \\
\hline Relative Density & Loose & Medium & Dense \\
\hline "Dry" or moist sand (range) & $4-13$ & $13-43$ & $43-86$ \\
\hline "Dry" or moist sand (adopted) & 8 & 25 & 64 \\
\hline Submerged sand (range) & 3-8 & $8-27$ & 27-54 \\
\hline Submerged sand (adopted) & 5 & 16 & 40 \\
\hline
\end{tabular}

where

$$
\begin{aligned}
& n_{h}=\text { constant of horizontal subgrade reaction for soldier beams } \\
& z=\text { depth below ground surface } \\
& B=\text { pile width }
\end{aligned}
$$

Values of $n_{h}$ for loose medium and dense sands are provided in Table 6.1. 


\subsubsection{Subgrade reaction for continuous walls}

Terzaghi (1955) indicated that, for continuous walls (i.e., sheet-pile walls and diaphragm walls) in stiff clay, the subgrade reaction $\left(k_{h}\right)$ can be assumed to be constant with depth, and taken as equal to

$$
k_{h}=\frac{64 S_{u}}{D}
$$

where $D$ is the effective contact dimension (Haliburton 1971), or interaction distance.

For the Bonneville temporary tieback wall, $D$ was taken as the average vertical distance between tendons. Dawkins (1994a) provided guidance for estimating the interaction distance. These guidelines are illustrated for singleand multiple-tieback anchor walls in Figure 6.4.

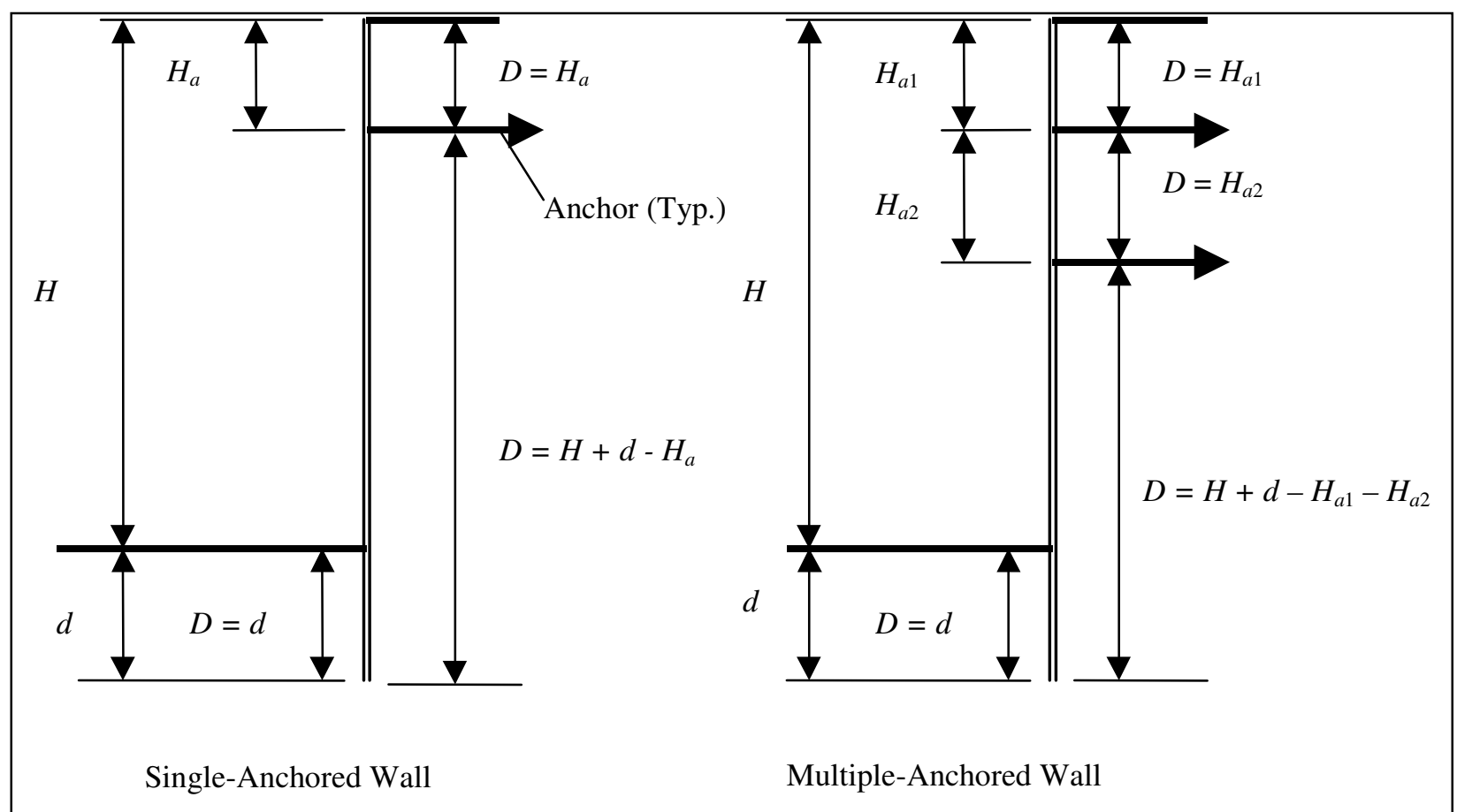

Figure 6.4 Initial estimates of interaction distances, $D$ (adapted from Dawkins 1994a)

For continuous walls in cohesionless soils, or in normally consolidated silts and clays, the coefficient horizontal subgrade reaction $\left(k_{h}\right)$ can be assumed to increase linearly with depth and taken as equal to 


$$
k_{h}=l_{h} \frac{z}{D}
$$

where

$$
\begin{aligned}
& l_{h}=\text { subgrade constant for continuous walls } \\
& z=\text { depth below ground surface }
\end{aligned}
$$

\begin{tabular}{|c|c|c|c|}
\hline \multicolumn{4}{|c|}{$\begin{array}{l}\text { Table } 6.2 \\
\text { Estimated Values of the Subgrade Constant for Continuous Wall } \\
\text { Systems in Moist and Submerged Sands (based on Table 4, } \\
\text { Terzaghi 1955) }\end{array}$} \\
\hline Soil Type - Sand & \multicolumn{3}{|c|}{ Subgrade Constant, $I_{h}(\mathrm{pci})$} \\
\hline Relative Density & Loose & Medium & Dense \\
\hline "Dry" or moist sand (adopted) & 3 & 9 & 23 \\
\hline Submerged sand (adopted) & 2 & 6 & 15 \\
\hline
\end{tabular}

Values of $l_{h}$ for loose, medium, and dense sands are provided in Table 6.2.

It should be noted that many computations have indicated that the moments and shears in soldier beams and continuous tieback wall systems are rather insensitive to the constant of horizontal subgrade reaction selected for the Winkler analysis. Upper and lower bound constants should be used to determine the impact on soldier beam and wall moments and shears. However, wall and soldier beam deflections are very sensitive to the constant of horizontal subgrade reaction used in the Winkler analysis. Therefore, it is difficult to obtain reasonable deflection values using this method of analysis.

\subsection{5 “Dry” versus submerged soils}

The constant of horizontal subgrade reaction for cohesionless sands is related to the relative density of the sand and the effective unit weight $\gamma$ of the sand (Terzaghi 1955). The constant of horizontal subgrade reaction for cohesionless soils will therefore be less for submerged soils than for dry or moist soils. The process of determining the coefficient of horizontal subgrade reaction, $k_{h}$, for a continuous wall constructed in cohesionless soils at partially submerged site is illustrated in Figure 6.5. 

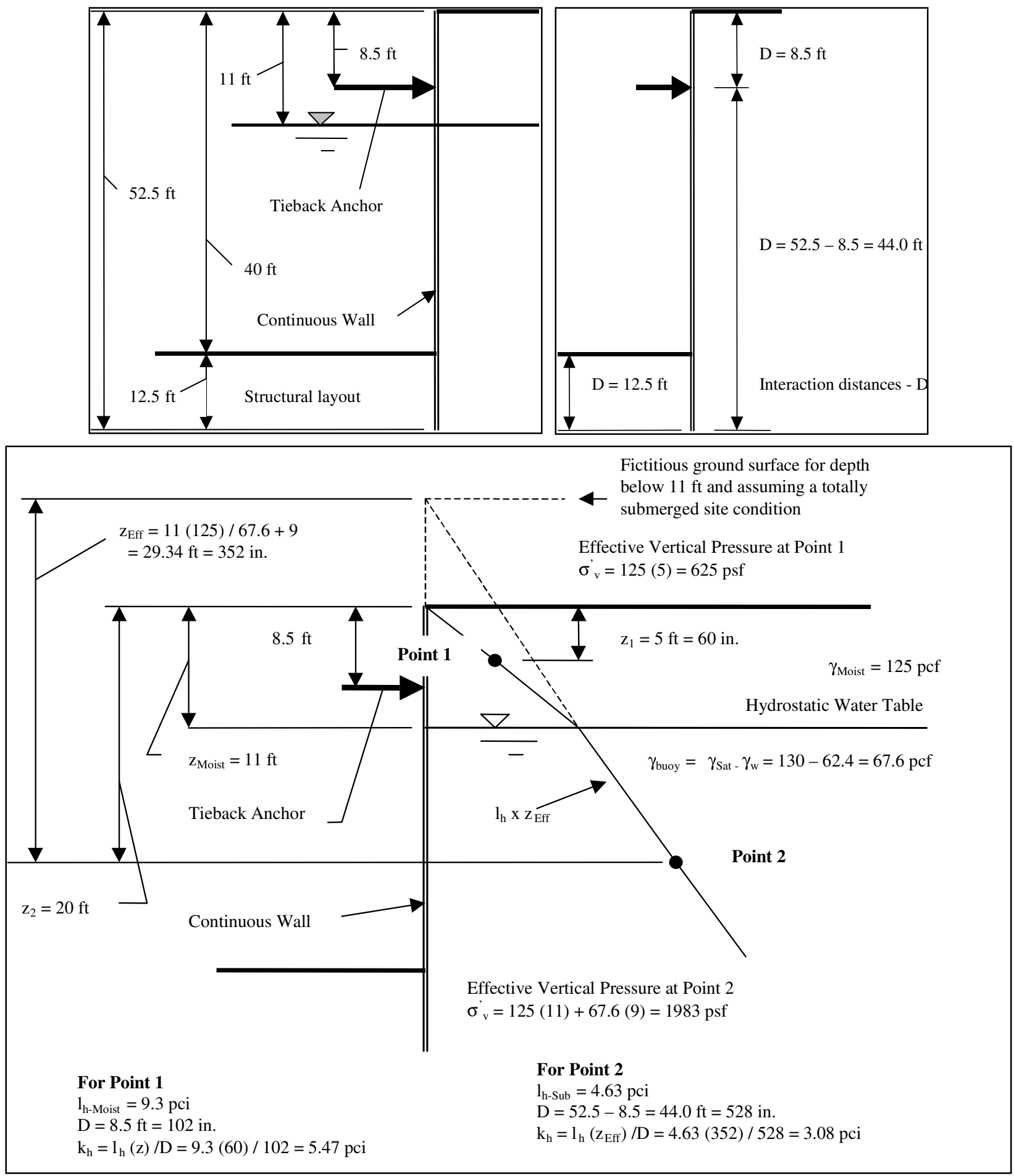

a. Solution based on equivalent submerged site methods

Figure 6.5. Subgrade reaction for a continuous wall, partially submerged conditions, hydrostatic water table in a granular soil $\left(\phi^{\prime}=35 \mathrm{deg}\right)($ Sheet 1 of 3$)$ 
Equation 12, Dawkins, 1994

$$
S_{a / p}=s_{a / p}\left(p_{v} / \gamma_{e}\right)
$$

Where: $\quad s_{\mathrm{a} / \mathrm{p}}=$ input soil active / passive stiffness coefficient, or $1_{\mathrm{h}}$ in the above example

$p_{\mathrm{v}}=$ effective vertical soil pressure at the calculation point, or $\sigma_{\mathrm{v}}$ in the above example $\gamma_{\mathrm{e}}=$ effective soil unit weight at the calculation point

For Point 1 (No interaction curve provided)

$S_{a / p}=s_{a / p}\left(p_{v} / \gamma_{e}\right)=9.3(625 / 144) /(125 / 1728)=558 p s i$

$K_{a / p}=S_{a / p} / D=558 / 102=5.47 p c i$

For Point 2 (See interaction curve below)

$S_{a / p}=s_{a / p}\left(p_{v} / \gamma_{e}\right)=4.63(1983 / 144) /(67.6 / 1728)=1629 p s i$

$K_{a / p}=S_{a / p} / D=1629 / 528=3.08 p c i$

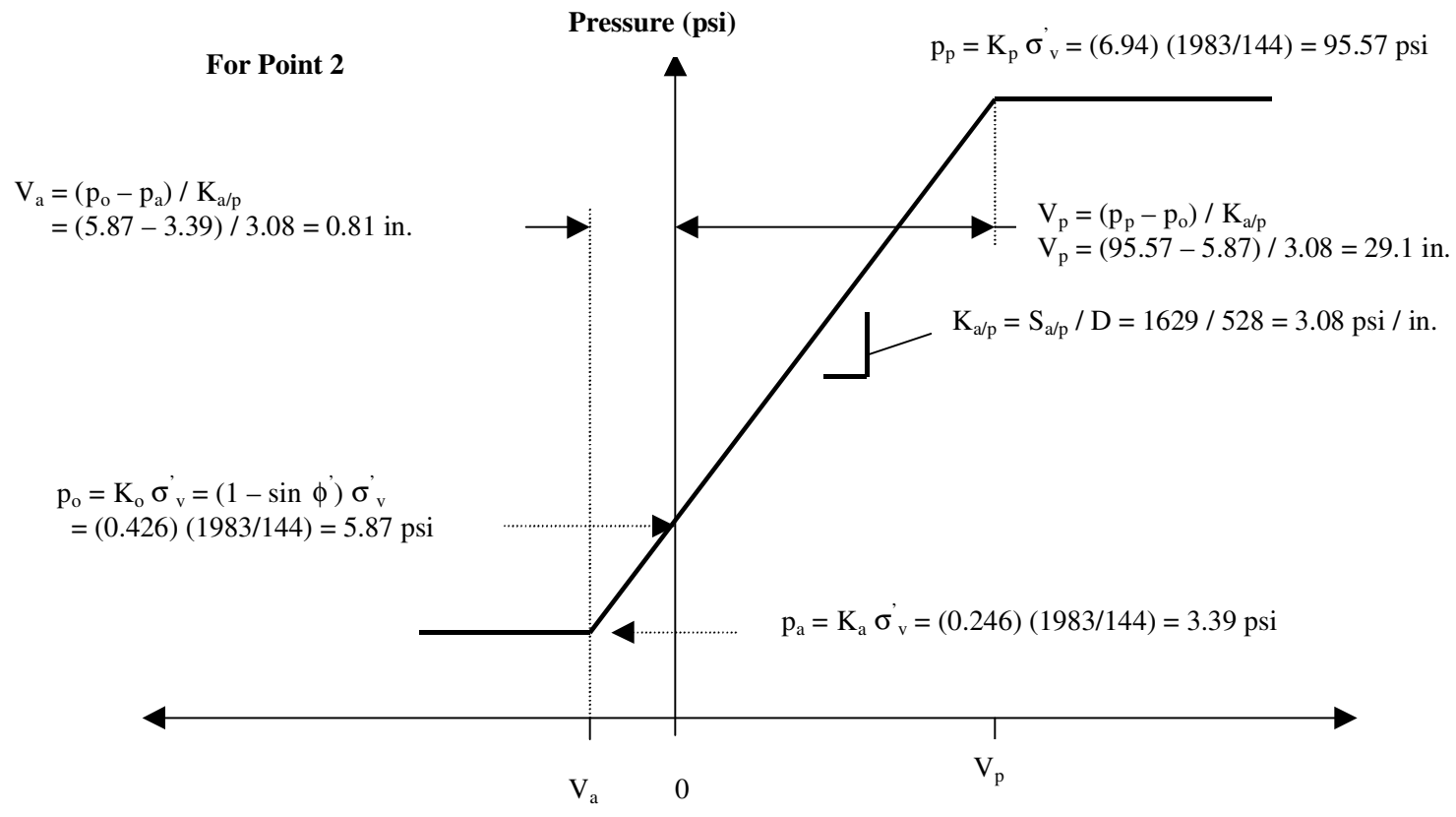

Displacement (inches)

Note: See Figure 6.5c for calculations used to determine the active, passive, and at-rest pressure coefficients

b. Solution based on effective stresses per CWALSSI nomenclature (Dawkins 1994a) (per Ebeling and Abraham SSI Course class notes)

Figure 6.5. (Sheet 2 of 3 ) 


\section{Active Earth Pressure Coefficient}

By Rankine equation assuming $\phi=35 \mathrm{deg}, \delta=0 \mathrm{deg}$, Equation 5 (Ebeling and Morrison 1992)

$$
K_{A}=\tan ^{2}(45-\phi / 2) \quad K_{A}=0.27
$$

By Coulomb equation assuming $\phi=35 \mathrm{deg}, \delta=17.5 \mathrm{deg}$; for level backfill $\beta=0$, for a vertical wall $\theta=0$ (Equation 16, Ebeling and Morrison 1992).

Note: Coulomb is only valid for $\delta \leq \phi^{\prime} / 2$

$$
K_{A}=\frac{\cos ^{2}(\phi-\theta)}{\cos ^{2} \theta \cos (\theta+\delta)\left[1+\sqrt{\frac{\sin (\phi+\delta) \sin (\phi-\beta)}{\cos (\delta+\theta) \cos (\beta-\theta)}}\right]^{2}} \quad K_{A}=0.246 \Leftarrow \text { Use }
$$

\section{Passive Earth Pressure Coefficient}

By Rankine equation assuming $\phi=35 \mathrm{deg}, \delta=0 \mathrm{deg}$, Equation 11 (Ebeling and Morrison 1992)

$$
K_{P}=\tan ^{2}(45+\phi / 2) \quad K_{P}=3.69
$$

By Coulomb equation assuming $\phi=35 \mathrm{deg}, \delta=17.5 \mathrm{deg}$; for level backfill $\beta=0$, for a vertical wall $\theta=0$ (Equation 29, Ebeling and Morrison 1992).

Note: Coulomb is only valid for $\delta \leq \phi^{\prime} / 2$

$$
K_{P}=\frac{\cos ^{2}(\phi+\theta)}{\cos ^{2} \theta \cos (\delta-\theta)\left[1-\sqrt{\frac{\sin (\phi+\delta) \sin (\phi+\beta)}{\cos (\delta-\theta) \cos (\beta-\theta)}}\right]^{2}} \quad K_{P}=7.357
$$

By Log Spiral assuming $\phi=35 \mathrm{deg}, \delta=17.5 \mathrm{deg}$, Figure 3.11 (Ebeling and Morrison 1992)

$K_{P}=R\left(K_{P}\right.$ for $\left.\delta / \phi=-1\right)=0.674(10.3)=6.94 \Leftarrow$ Use

\section{At-Rest Earth Pressure Coefficient}

An adequate value of the at-rest pressure coefficient $\left(K_{o}\right)$ can be estimated using Jaky's equation for normally consolidated sands (not including compaction effects).

$K_{o}=1-\sin \phi^{\prime}=1-\sin 35 \mathrm{deg}=0.426 \Leftarrow$ Use

\section{Active, passive, and at-rest pressure coefficients}

Figure 6.5. (Sheet 3 of 3 ) 
The differences in the subgrade constant, $l_{h}$, for moist and submerged soils should be noted. The interaction distance, $D$, is problem dependent and for this particular example happens to be different for the two points under consideration. In actuality the value of $k_{h}$ in cohesionless soil is a function of the effective vertical stress, $\sigma_{v}^{\prime}$. However, in a uniform soil layer the value of $k_{h}$ at Point 2 (or any other point below the water table) for the submerged condition can be determined using a fictitious depth, $z_{E f f}$, which would be the depth of a totally submerged soil providing the same effective confining pressure. This process is illustrated in Figure 6.5a.

Figure $6.5 \mathrm{~b}$ provides calculations for the coefficient of horizontal subgrade reaction, $k_{h}$, using the effective vertical stress method in accordance with the CWALSSI nomenclature (Dawkins 1994a) and SSI course class notes. ${ }^{1}$ The effective vertical stress method should be used for nonuniform soil conditions. The maximum and minimum resistance used to define the plastic plateaus of the elastoplastic Winkler curve for submerged and partially submerged soils at depth $z$ will depend on the effective vertical stress, $\sigma_{v}^{\prime}$, and on the values for the passive and active earth pressure coefficients.

Three methods are available for calculating these coefficients: the Rankine method, the Coulomb method, and the logarithmic spiral failure surface analysis. These methods are described in Ebeling and Morrison (1992) and illustrated in Figure 6.5c. In Figure 6.5c, the Coulomb method was selected for determining the active earth pressure coefficient, $K_{a}$, and the logarithmic spiral failure surface analysis for determining the passive earth pressure coefficient, $K_{p}$. The at-rest pressure coefficient, $K_{o}$, was determined using Jaky's equation for normally consolidated sands. Methods for calculating the displacements associated with the intersection of the linear elastic and plastic regions of the $R-y$ curve are illustrated in Figure 6.5b.

\subsubsection{Soil springs}

In general practice, the SSI analysis for tieback wall systems must consider the nonlinear characteristics of the soil springs. This is usually accomplished with springs that use ideal elastoplastic behavior to capture the nonlinear response, although more exact representations of the nonlinear soil response have been developed by the American Petroleum Institute (Murchison and O'Neill 1984, O'Neill and Murchison 1983).

A typical elastoplastic soil pressure curve is shown in Figure 6.6. This curve is generally constructed by the Coefficient of Subgrade Reaction Method, the Reference Deflection Method, or the Pfister Method as described below. Earth pressure-deflection springs below the excavation for a discrete wall system are different from those of a continuous wall system. The earth-pressure defection springs for discrete wall systems must include three-dimensional (3-D) effects, similar to a laterally loaded pile (Weatherby, Chung, Kim, and Briaud 1998).

\footnotetext{
${ }^{1}$ R. M. Ebeling and K. Abraham. (1999). "Soil-structure interaction—class notes," U.S. Army Engineer Research and Development Center, Vicksburg, MS.
} 
To be consistent with the terminology used in the FWHA reports, the elastoplastic soil response curves used for the evaluation of continuous and discrete tieback wall systems will be designated as $R-y$ curves. The active and passive loads defining the $R-y$ curve plastic regions for continuous wall systems use active and passive pressures over a unit width of wall. The active and passive loads defining the $R-y$ curve plastic regions of discrete wall systems use active and passive pressures multiplied by the soldier beam spacing. $R-y$ curves are used in one of two different beam-column computer programs, BMCOL76 or CBEAMC. Both programs model the earth pressure-deflection behavior of the system identically and give the same results for similar problems. The soil spring designations shown in Figure 6.6 follow the conventions of CBEAMC (Dawkins 1994b), Example 4.

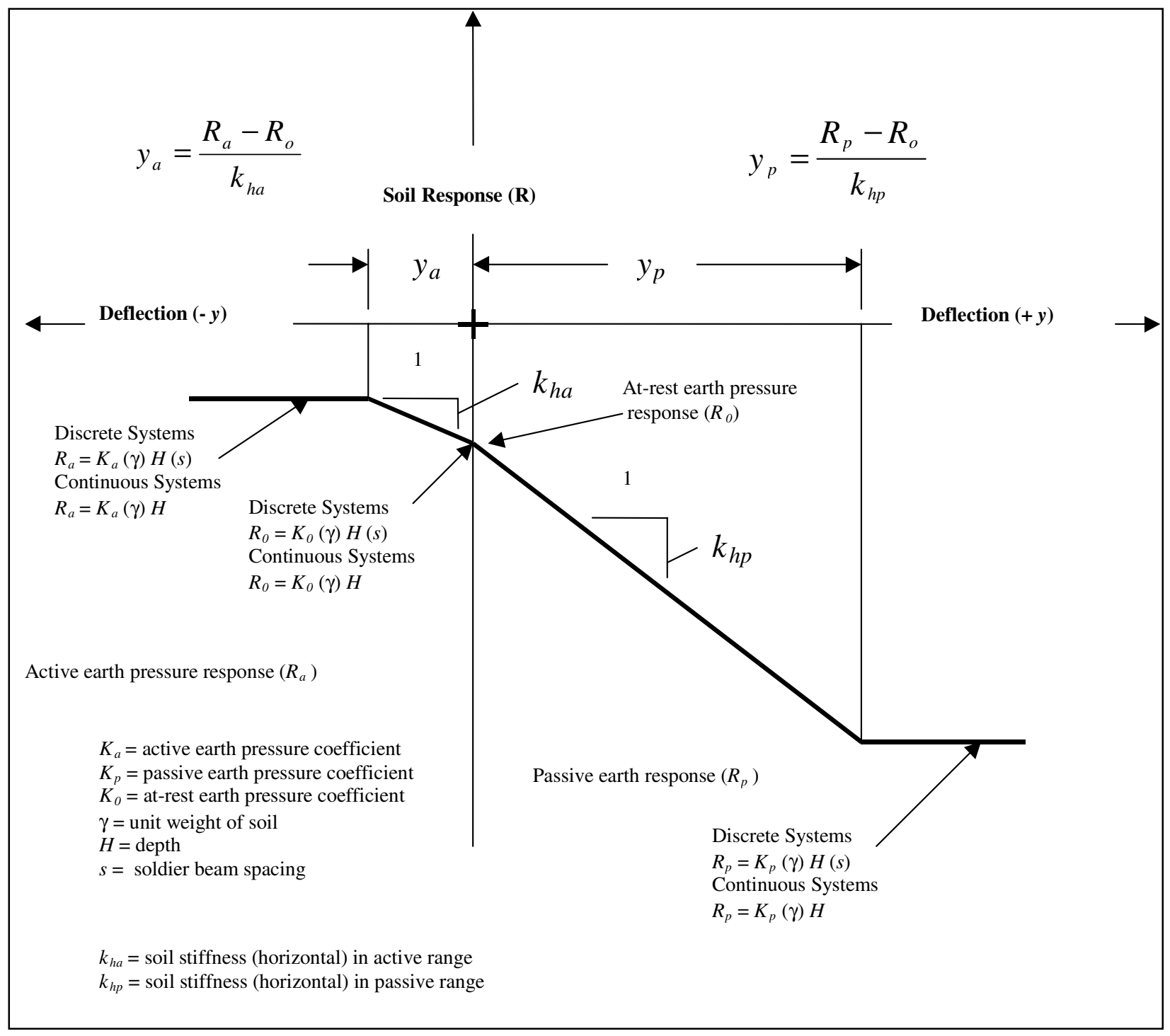

Figure 6.6. Idealized elastoplastic earth response-deflection $(R-y)$ curve (right side soil spring per CBEAMC convention) (Dawkins 1994b) 
6.2.6.1 Coefficient of subgrade reaction method. In the coefficient of subgrade reaction method, the linear elastic portion of the $R-y$ curve is developed using constants of subgrade reaction values or subgrade constants equal or similar to those described in Tables 6.1 and 6.2. A single constant of subgrade reaction or subgrade constant may be used to describe the linear elastic range between active and passive pressure, or different values may be used to define the region between at-rest and active and between at-rest and passive. Recall that at-rest pressure corresponds to zero deflection of the soil behind the retaining wall.

For the coefficient of subgrade reaction method, the elastic stiffness is determined directly for each point from the top of the wall to the toe. In practice, however, values are provided for discrete points usually representing a change in soil properties or change in effective stress. The computer program then generates a series of infinitely closely spaced soil springs with a coefficient of subgrade reaction $\left(k_{h}\right)$ varying linearly between the values described at the discrete points. In this way the coefficient of subgrade reaction can either vary linearly with depth (cohesionless soils, normally consolidated silts, and normally consolidated clays) or be constant with depth (cohesive soils). The deflections representing the change from linear elastic to active $\left(y_{a}\right)$ and linear elastic to passive $\left(y_{p}\right)$ are determined by the following equations:

$$
\begin{gathered}
y_{a}=\frac{P_{0}-P_{a}}{k_{h a}} \\
y_{p}=\frac{P_{p}-P_{0}}{k_{h p}}
\end{gathered}
$$

where

$y_{a}=$ deflection representing the change from linear elastic to active

$P_{0}=$ at-rest stress state for soil spring (at zero deformation)

$P_{a}=$ active stress state for soil spring

$k_{h a}=$ horizontal coefficient of subgrade reaction - at-rest to active (soil spring active state elastic stiffness)

$y_{p}=$ deflection representing the change from linear elastic to passive

$P_{p}=$ passive stress state for soil spring

$k_{h p}=$ horizontal coefficient of subgrade reaction - at-rest to passive (soil spring passive state elastic stiffness)

Often $k_{h a}$ and $k_{h p}$ are assumed to be equal (constant slope for linear elastic region). Active and passive stress states for the soil springs are usually based on conventional earth pressure theory (Rankine or Coulomb). However, passive soil failures related to the toe region of discrete soldier beam systems require the consideration of special failure mechanisms that cannot be predicted by 
conventional earth pressure theory. These failure mechanisms and their associated passive state capacities are described in Chapter 8.

6.2.6.2 Reference deflection method. With cohesionless soils, the limit state pressures (active and passive pressures) increase linearly with depth. Based on information presented above with respect to $p-y$ curves, the displacements required to develop active or passive pressure will also increase linearly with depth. This suggests that the displacements required to generate active or passive conditions should be constant with depth, and gives rise to the reference deflection method for developing soil spring elastoplastic $R-y$ curves. The reference deflections are those necessary to mobilize active and passive soil resistance, and depend on soil type.

In Weatherby, Chung, Kim, and Briaud (1998), the reference deflections for sand were based on measurements obtained from the Texas A\&M full-scale wall tests. The wall tested was $7.6 \mathrm{~m}(25 \mathrm{ft})$ high and consisted of soldier beams and wood lagging supported by one and two rows of pressure-injected ground anchors. The wall was constructed in a homogenous sand deposit. Wall design and construction is described in Section 2.2.2 of Weatherby, Chung, Kim, and Briaud (1998). Earth pressures acting on the soldier beams were calculated by double differentiation of bending moments determined from strain gauge data. Earth pressures were plotted against measured lateral displacements. The deflection required to fully mobilize active earth pressure was found to be $0.13 \mathrm{~cm}$ (0.05 in.). The deflection required to fully mobilize passive earth pressure was assumed to be $1.27 \mathrm{~cm}$ (0.5 in.). Earth pressure-displacement relationships for the test wall are described in Section 3.2.2 of Weatherby, Chung, Kim, and Briaud (1998).

Reference deflections for clay were assumed and verified by comparing the predicted behavior with case history results (Weatherby, Chung, Kim, and Briaud 1998). In the horizontal coefficient of subgrade reaction method, the deflections $y_{a}$ and $y_{p}$ are determined conceptually using the procedure outlined in Figure 6.5. The reference deflection method differs from the horizontal coefficient of subgrade reaction method in that the deflections $y_{a}$ and $y_{p}$ are established values (dependent on soil type), rather than dependent on predetermined values of soil spring stiffness. In the reference deflection method, the soil spring stiffness is a by-product of the reference deflections and soil maximum and minimum loads. An active reference deflection of $0.13 \mathrm{~cm}(0.05 \mathrm{in}$.) and a passive reference deflection of $1.27 \mathrm{~cm}(0.5 \mathrm{in}$.) have been used in the development of $R-y$ curves for cohesionless soils. The process is illustrated in Figure 6.7 for continuous wall systems and for discrete (soldier beam) systems.

The same FHWA report (Weatherby, Chung, Kim, and Briaud 1998) provides reference deflections for soldier beam and continuous wall tieback systems constructed in clay. Figure 6.8 shows the typical $R-y$ curve construction using the reference deflection method for different portions of a continuous anchored wall supporting a cohesive soil. Both the undrained and drained cases are illustrated. 

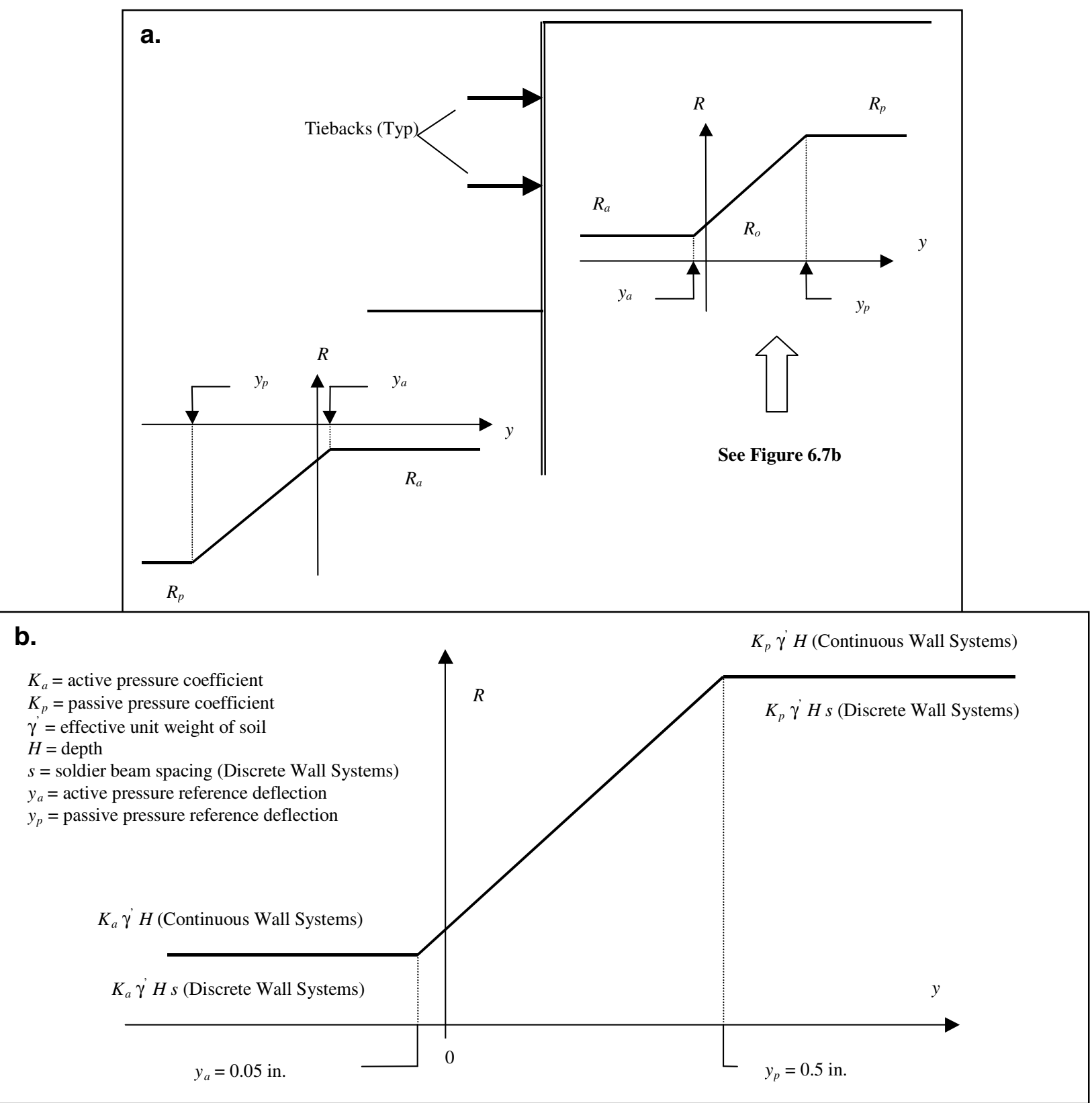

Figure 6.7. Diagram illustrating $R-y$ curves for tieback walls in cohesionless soils (general $R-y$ curve system per Weatherby, Chung, Kim, and Briaud 1998) 


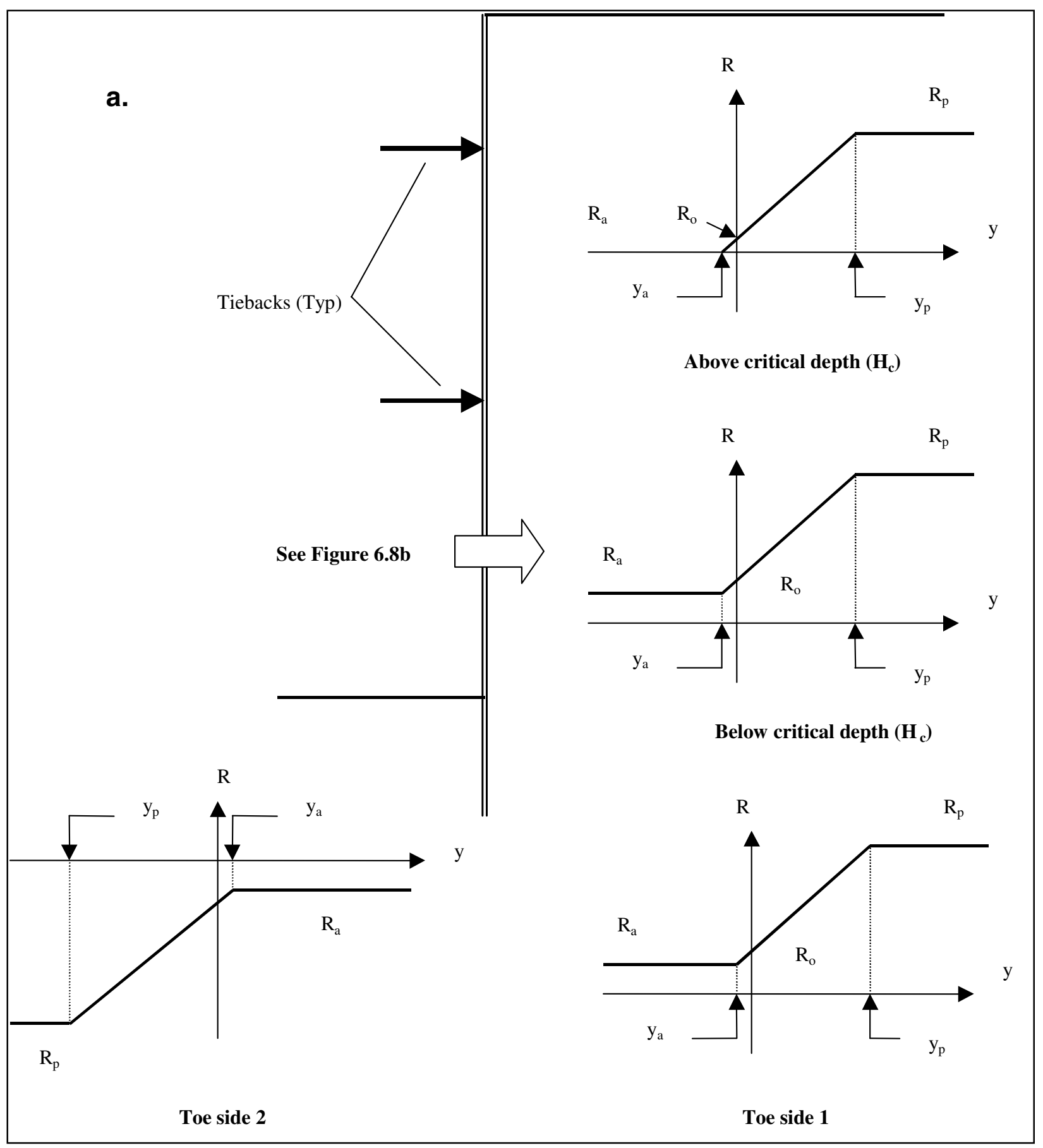

Figure 6.8. Diagram illustrating $R-y$ curves for continuous tieback walls in cohesive soils (general $R-y$ curve system per Weatherby, Chung, Kim, and Briaud 1998) (Continued) 


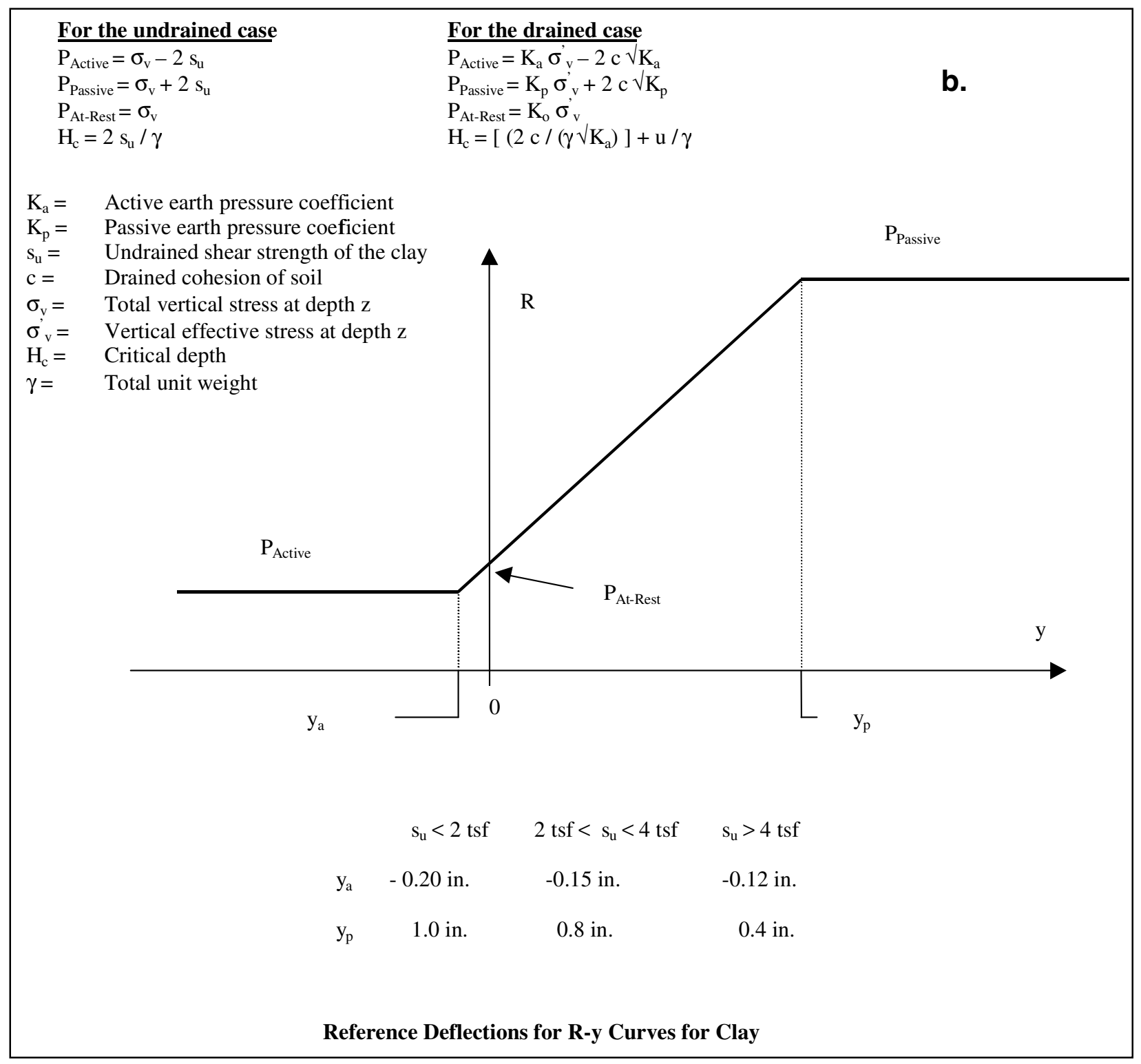

Figure 6.8. (Concluded)

The soil resistance-deflection $(R-y)$ curves for the toe of a soldier beam (discrete wall system) in clay are different from the $R-y$ curves for a continuous wall system in clay. The $R-y$ curves for soldier beam toes in clay are based on laterally loaded pile tests performed by Reese, Cox, and Koop (1975). The development of a typical lateral resistance-deflection curve for a single pile in stiff clay is shown in Figure 6.9a. The $P$ - $y$ curve in Figure 6.9a is symmetrical since the ground surface is horizontal. $R-y$ curves for the toe of a soldier beam in clay will be nonsymmetrical, since the ultimate resistance, $P_{u}$, depends on overburden depth. Figure $6.9 \mathrm{~b}$ illustrates how an $R-y$ curve would be developed from the $P-y$ curve of Figure 6.9a. The reference deflections required to define the elastic portion of the $R-y$ curve are also provided in Figure 6.9. These deflections were developed from laterally loaded pile practice and verified/ modified after comparing predicted bending moments with measured bending moments for the case histories presented in Weatherby, Chung, Kim, and Briaud (1998). 


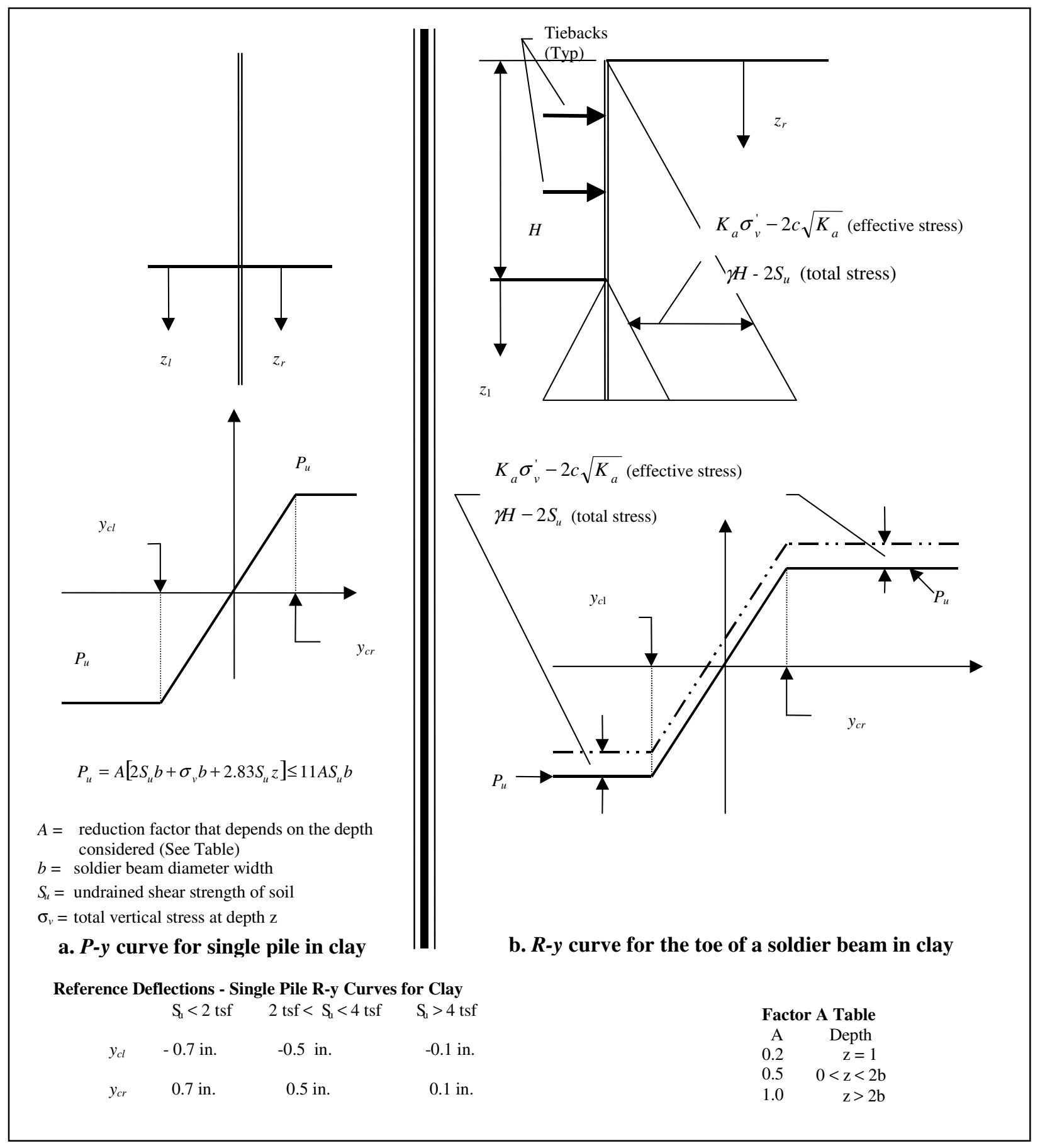

Figure 6.9. Diagram illustrating $P-y$ and $R-y$ single pile curves in clay

Parametric studies (Weatherby, Chung, Kim, and Briaud 1998) showed that the bending moments in flexible wall systems were not very sensitive to the slope of the $R-y$ curve (stiffness of the nonlinear spring). The parametric studies did show that the moments were sensitive to the values of the maximum and minimum resistance used to define the plastic plateaus of the elastoplastic curve. The studies indicated that the active resistance had to be reduced by \pm 50 percent 
to obtain results comparable to those of the test wall. Since the test wall was a discrete soldier beam system, the pressures acting on the back face will be considerably smaller than active Rankine or Coulomb earth pressures due to arching effects. The opposite is true for passive pressure resistance. The use of an active resistance pressure lower than Rankine/Coulomb, and a passive resistance pressure greater than Rankine/Coulomb, is therefore justified with respect to the Winkler spring analysis of discrete tieback wall systems. However, the actual relationship between arching effects and active pressure resistance is unknown.

6.2.6.3 Pfister method. Pfister developed relationships between soil strength and horizontal subgrade reaction for stiff continuous diaphragm walls (Pfister, Evers, Guillaud, and Davidson 1982). Soletanche uses these relationships to develop elasto-plastic soil springs for use in beam on elastic foundation analyses. The beam on elastic foundation analyses are used by Soletanche to evaluate tieback wall behavior for various stages of construction. The Pfister relationships between soil strength and horizontal subgrade reaction are presented in Figure 6.10. They are generally used where no information other than the shear parameters of the loaded soils is available. The procedure used to develop elasto-plastic soil springs for a beam on elastic foundation analysis is illustrated in Pfister, Evers, Guillaud, and Davidson (1982, pp 138 and 141) and described below.

In the illustration, two sets of soil parameters were provided by the soils engineer with the understanding that either set (I or II) could be used for the analysis. The soil parameters are as indicated in Table 6.3.

\begin{tabular}{|l|l|l||}
\hline \hline \multicolumn{2}{|l||}{$\begin{array}{l}\text { Table 6.3 } \\
\text { Soil Parameters for Pfister Method Illustration }\end{array}$} \\
\hline \hline Soil Parameter & Soil I & Soil II \\
\hline \hline Angle of internal friction, $\phi$ & $25 \mathrm{deg}$ & $35 \mathrm{deg}$ \\
\hline Cohesion, $C$ & $300 \mathrm{psf}\left(1.5 \mathrm{t} / \mathrm{m}^{2}\right)$ & $0 \mathrm{psf}\left(0 \mathrm{t} / \mathrm{m}^{2}\right)$ \\
\hline
\end{tabular}

From Figure 6.10:

$$
\begin{aligned}
& \text { For Soil I } \quad k_{h}=2,200 \mathrm{t} / \mathrm{m}^{3}\left(138 \mathrm{k} / \mathrm{ft}^{3}\right) \\
& \text { For Soil II } \quad k_{h}=4,000 \mathrm{t} / \mathrm{m}^{3}\left(250 \mathrm{k} / \mathrm{ft}^{3}\right)
\end{aligned}
$$

Since the SPT results for the soil increased slightly with depth, Pfister, Evers, Guillaud, and Davidson (1982) used judgment in assigning the following horizontal subgrade reaction parameters:

- A coefficient of subgrade reaction was selected to be equal to $2,000 \mathrm{t} / \mathrm{m}^{3}\left(125 \mathrm{k} / \mathrm{ft}^{3}\right)$ at an effective vertical soil pressure of $0 \mathrm{psf}$, and 


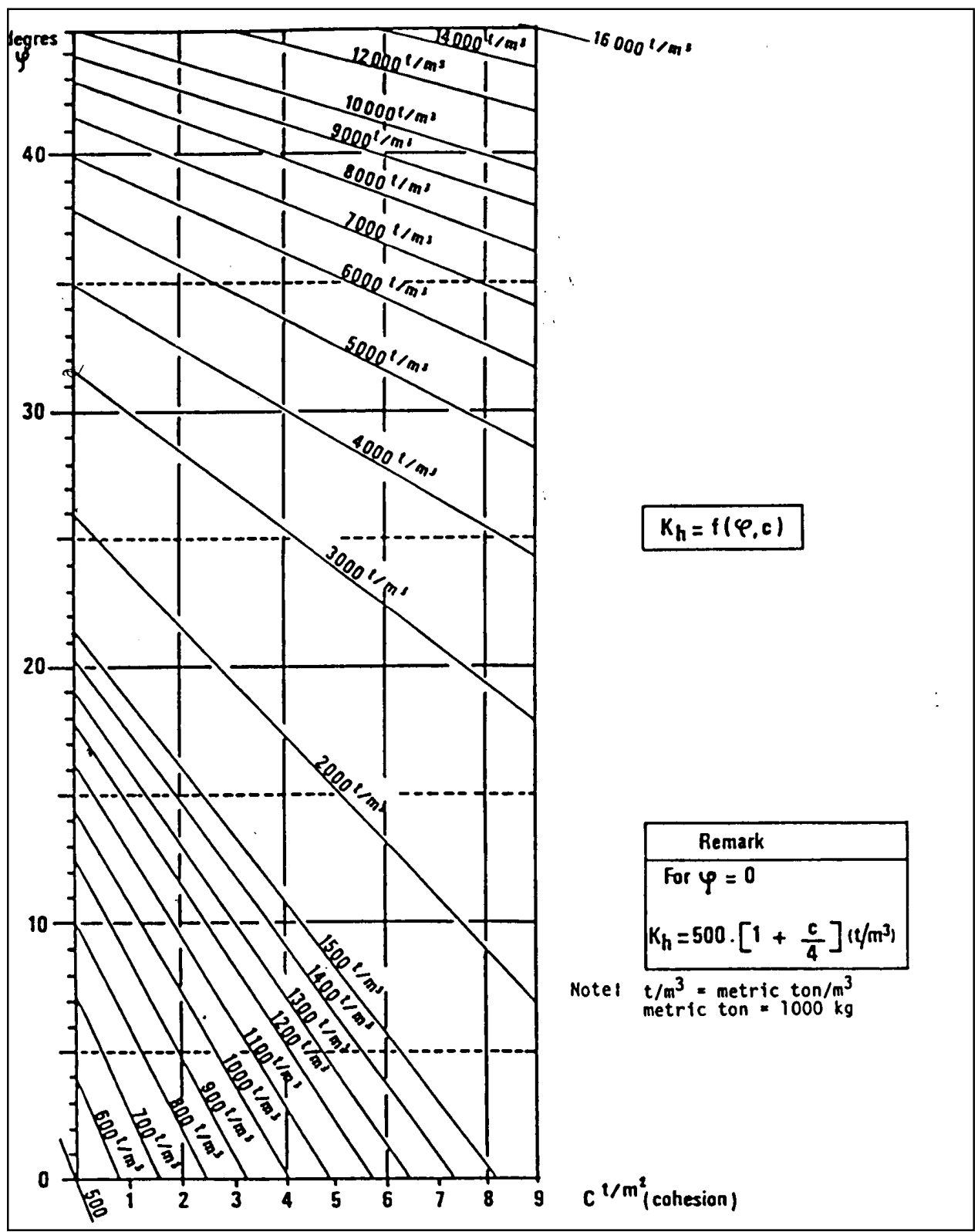

Figure 6.10. Horizontal subgrade moduli, $k_{h}$ (after Pfister, Evers, Guillaud, and Davidson 1982)

- The coefficient of subgrade reaction was selected to increase at a rate of $200 \mathrm{t} / \mathrm{m}^{3}\left(12.5 \mathrm{k} / \mathrm{ft}^{3}\right)$ for each increase in effective vertical soil pressure of $1 \mathrm{t} / \mathrm{m}^{2}\left(0.207 \mathrm{k} / \mathrm{ft}^{2}\right)$.

Illustrating the development of the elasto-plastic soil spring for an effective vertical soil pressure of $1,000 \mathrm{psf}$ :

- The coefficient of subgrade reaction, $k_{h}$, is $125+12.5(1 / 0.207)=$ $185 \mathrm{k} / \mathrm{ft}^{3}$. 
- Assuming that the active, at-rest, and passive pressure coefficients are $0.271,0.430$, and 7.346 , respectively, the displacements for the start of the active pressure and passive pressure plateaus are

$$
\begin{aligned}
& \Delta_{a}=\frac{\left(p_{o}-p_{a}\right)}{k_{h}}=\frac{(0.430-0.271)}{185}=0.0009 \mathrm{ft}=0.0103 \mathrm{in} . \\
& \Delta_{p}=\frac{\left(p_{p}-p_{o}\right)}{k_{h}}=\frac{(7.346-0.430)}{185}=0.0374 \mathrm{ft}=0.4488 \mathrm{in} .
\end{aligned}
$$

Using this information, the elasto-plastic soil spring for an effective vertical soil pressure of $1,000 \mathrm{psf}$ can be constructed and other elasto-plastic soil springs for different effective vertical soil pressures constructed in a similar fashion.

\subsection{Prestressed Anchor Springs}

The load-deflection $(T-y)$ curve for a prestressed tieback anchor for final design load conditions is shown in Figure 6.11 and described in Weatherby (1998). This represents the load-deflection response after all tendon losses have occurred and assumes that, when the wall has a zero displacement, the tieback anchor is at a design stress equal to 60 percent of its ultimate stress. With highstrength steel anchors, there is no clearly defined yield plateau. For plain and deformed prestressing bars, the yield stress $\left(f_{y}\right)$ is assumed to be 95 percent of the ultimate stress. For seven-wire low-relaxation prestressing strand, the minimum yield stress $\left(f_{y}\right)$ is assumed to be at 1 percent elongation, or at 1,675 MPa (243 ksi) for an ultimate stress of 1,862 MPa (270 ksi), and 1,551 MPa (225 ksi) for an ultimate stress of $1,724 \mathrm{MPa}(250 \mathrm{ksi})$. The linear elastic portion of the $T-y$ curve can be developed using the yield displacement $\left(y_{y}\right)$, the tendon yield strength $\left(T_{y}\right)$, and the tendon elastic stiffness $\left(k_{T}\right)$.

The yield displacement $\left(y_{y}\right)$ is equal to

$$
y_{y}=-\frac{\left(f_{y}-0.60 f_{y}\right) L_{u}}{E_{s}} \cos \alpha
$$

The tendon yield strength $\left(T_{y}\right)$ is

$$
T_{y}=A_{s} f_{y} \cos \alpha
$$

and the tendon elastic stiffness $\left(k_{T}\right)$ is

$$
k_{s}=\frac{A_{s} E_{s}}{L_{u}} \cos \alpha
$$


where

$L_{u}=$ effective elastic length of the anchor (assumed to be equal to the unbonded length + one-half the bond length)

$\alpha=$ anchor inclination with horizontal

$E_{s}=$ Young's modulus for anchor tendon

$A_{s}=$ area of anchor tendon

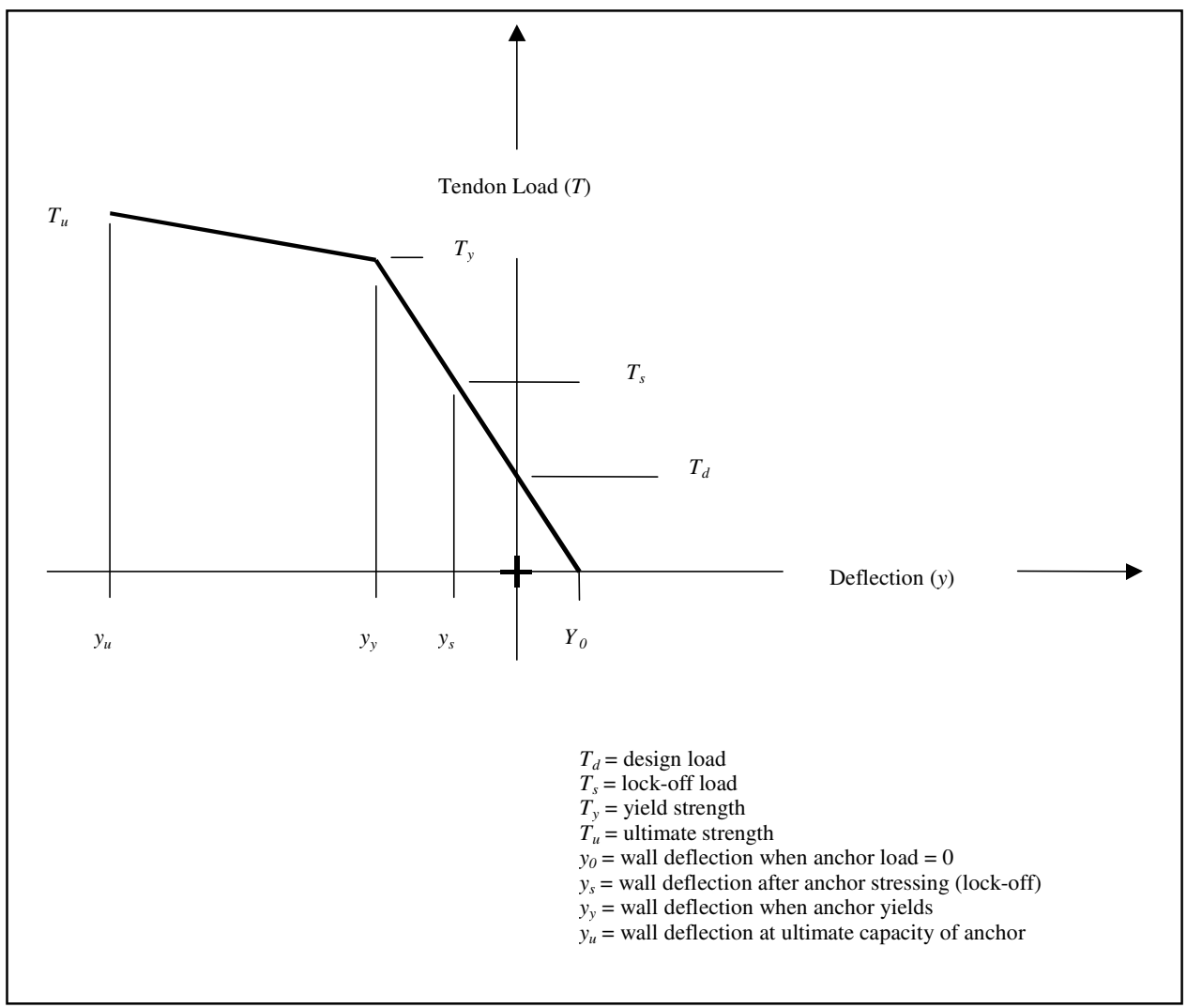

Figure 6.11. Ground anchor $T-y$ curve (adapted from Figure 59, Weatherby 1998)

The tensile load region of the $T-y$ curve beyond yield can be assumed as horizontal (constant tensile force) to ultimate. This would be similar to the elastoplastic behavior assumed for the soil. Or, the actual tensile force associated with ultimate stress capacity $\left(T_{u}=A_{s} f_{u} \cos \alpha\right)$, along with the ultimate displacement, $y_{u}=-\frac{\left(f_{u}-0.60 f_{y}\right) L_{u}}{E_{s}} \cos \alpha$, can be used to construct this region of the $T-y$ curve. This approach would allow consideration of strain hardening in the post-yield region. The load-deflection $(T-y)$ curve used for prestressed tieback anchors in a construction sequencing analysis differs from that described above for final design load conditions. Construction sequencing analyses using Winkler spring analysis are discussed below. 


\subsection{Tieback Wall Toe Evaluation}

The Winkler spring approach can also be used to evaluate the pressure distribution and displacement of the embedded toe portion of the wall, as well as the effects of toe behavior with respect to the overall load-displacement performance of the tieback wall (Weatherby 1998).

The Winkler soil spring system for a tieback wall toe evaluation is illustrated in Figure 6.12. In this figure, discrete springs (one for the backside embedded portion of the wall and one for the front side embedded portion of the wall) are shown. These springs represent infinitely closely spaced soil springs that can have a stiffness that increases linearly with depth to approximate the behavior of cohesionless soils, normally consolidated silts, and normally consolidated clays, or may be constant with depth to represent the approximate behavior of cohesive soils. In this particular model, apparent earth pressures are used as loads to capture the load-displacement behavior of the part of the wall above the bottom of the excavation. The procedure for developing soil and anchor springs is as described above, except that the maximum resistances for the soil $R-y$ curves are usually computed using the relationships developed by Wang and Reese (1986), as described in Chapter 8.

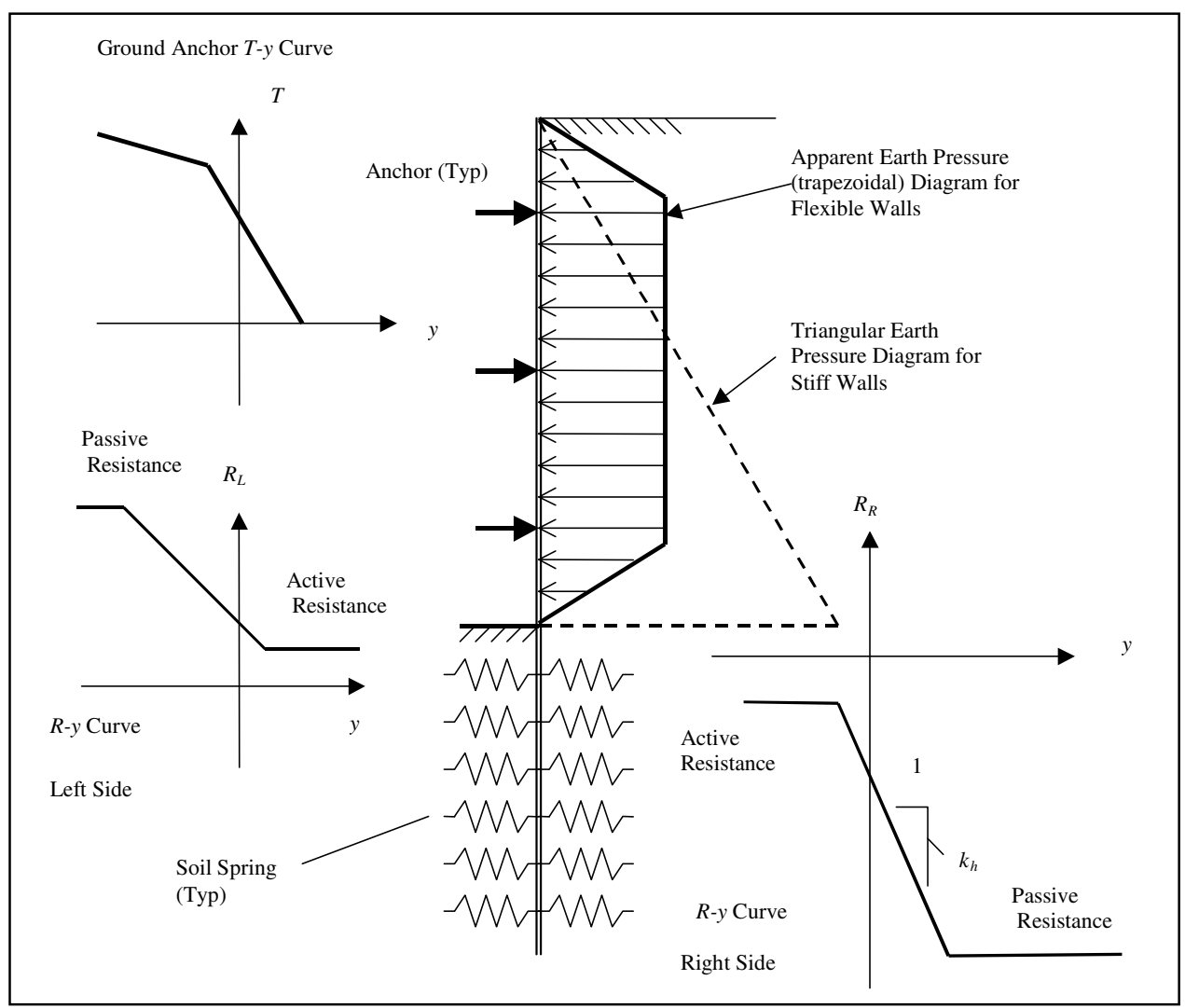

Figure 6.12. Winkler spring model for toe analysis (adapted from Figure 56, Weatherby 1998) 


\subsection{Final Excavation Evaluation Using Winkler Spring Analyses (Illustrated Using the Temporary Tieback Wall at Bonneville as an Example)}

\subsubsection{General}

Tieback wall behavior with respect to the completed wall is often accomplished using a Winkler spring analysis using a "one-step construction" model that does not consider construction sequencing. This approach was used to evaluate the performance of the temporary tieback wall for the Bonneville Navigation Lock (Munger, Jones, and Johnson 1991). To facilitate construction of a new navigation lock at Bonneville Dam, a 15.2-m (50-ft)-high continuous reinforced concrete tieback wall was built to temporarily retain the ground adjacent to the lock excavation. A typical section through the wall is shown in Figure 6.13.

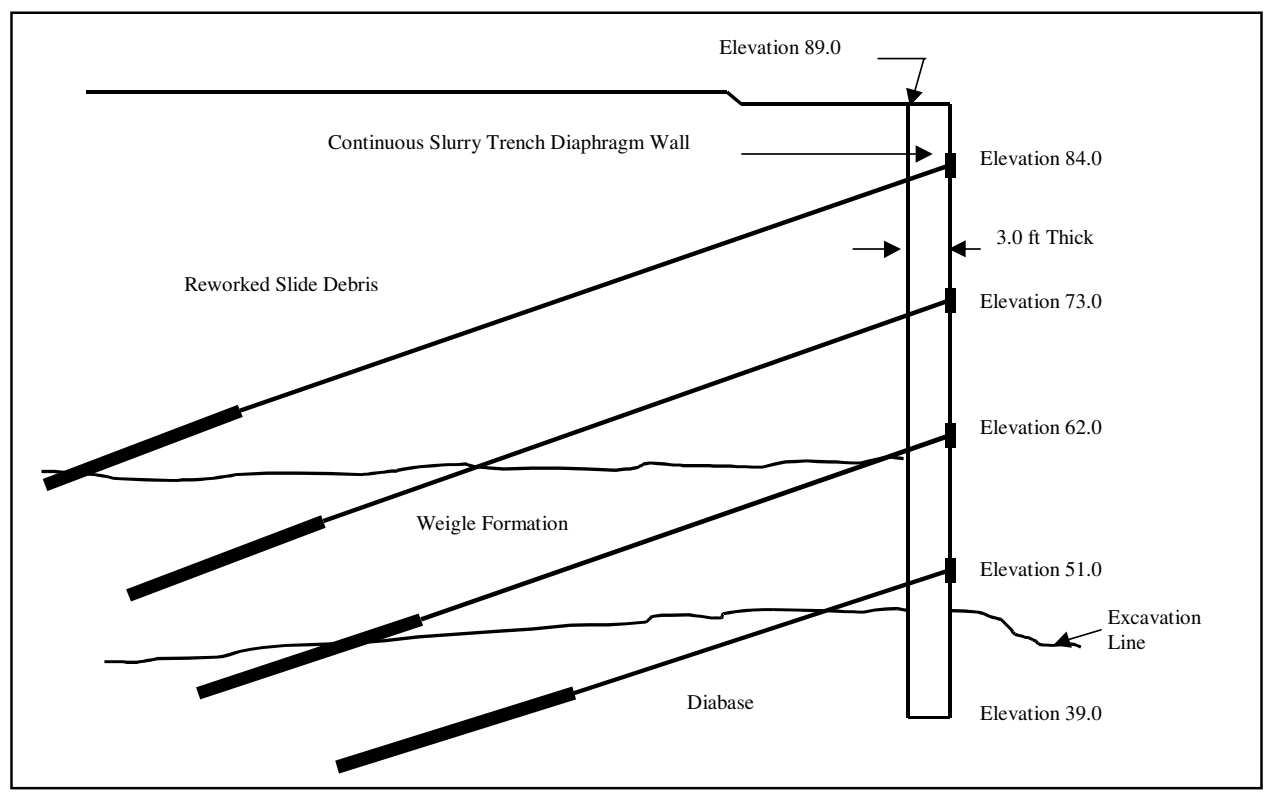

Figure 6.13. Bonneville Navigation Lock temporary tieback wall

The ground adjacent to the lock supports the Union Pacific Railroad. The design of this wall is of particular interest with respect to Corps tieback wall construction because

- Displacement performance objectives as well as collapse prevention performance must be satisfied.

- Tieback anchors must be designed to accommodate at-rest soil pressures rather than active soil pressures to limit displacements of the ground behind the wall to acceptable levels. 
a. A continuous wall system that is stiff rather than flexible must be used to limit displacements of the ground behind the wall to acceptable levels.

The Bonneville tieback wall Winkler spring evaluation used to evaluate the performance of the tieback wall is described in following paragraphs.

\subsubsection{Tieback wall evaluation using Winkler spring analysis}

The performance of the Bonneville Navigation Lock temporary tieback wall was evaluated using the computer program CBEAMC (Dawkins 1994b), which models SSI using beam-column structures with 1-D linear and nonlinear supports based on the Winkler soil spring model. Initial loads on the wall were at-rest soil loads with the tendons stressed to a design load based on at-rest pressure conditions (i.e., at zero deformations). The stiffness of the soil springs used in the analysis was a function of Terzaghi's subgrade constant $\left(l_{h}\right)$, with the coefficient of horizontal subgrade reaction $\left(k_{h}\right)$ assumed to increase linearly with depth and taken as equal to

$$
k_{h}=l_{h} \frac{z}{D}
$$

A value for the interaction zone depth $(D)$ was taken as the average vertical distance between tendons in accordance with Haliburton (1971). The horizontal spacing between anchors was equal to $3.35 \mathrm{~m}(11.0 \mathrm{ft})$. The wall was idealized as a 3.4-m (11.0-ft)-wide beam spanning vertically between tieback anchors. Beam properties and soil springs were developed for the 11.0-ft-wide beam section and used as input to the CBEAMC Program. No considerations were given to plate action (3-D) effects.

The water table was below the base of the wall. Nonlinear soil springs were developed using the "coefficient of horizontal subgrade reaction method" described above with the displacements marking the start of nonlinear behavior determined in accordance with Equations 6.6a and 6.6b. The wall toe was embedded in rock, and rock-springs were developed as "passive pressure only" springs. The modulus of elasticity of the rock was used as the spring elastic stiffness, with the displacement marking passive nonlinear behavior determined as for soil using a maximum passive load equal to 80 percent of the unconfined compressive strength of the rock. The effective length of the tendons was assumed to be equal to the unbonded length plus one-half the bonded length. The design required that the tendon load not exceed 80 percent of the ultimate strength. The elastic spring representing the tendon had an elastic stiffness based on the elastic modulus of steel, the cross-sectional area of the tendon, and the effective length of the tendon. Beyond 80 percent of ultimate, the tendon load was zero, representing tendon failure. Tendon behavior in the inelastic range $\left(T_{y}\right.$ to $T_{u}$ in Figure 6.11) was not permitted in the Bonneville tieback wall analysis. 


\subsubsection{Winkler spring analysis results}

The tieback wall at Bonneville Dam was heavily instrumented to monitor deflections, wall moments, and tendon loads. The initial predictions of the wall deflections and moments were reasonably close to measured values. Increasing the subgrade coefficient of subgrade reaction by a factor of 5 brought the Winkler spring analysis deflections to close proximity with measured values, but did not appreciably alter the magnitude of the calculated moments. This demonstrates that wall moments and shears are not too sensitive to the value used for the coefficient of subgrade reaction, but that the opposite is true with respect to displacements. The behavior of the Bonneville tieback wall was also evaluated by Weatherby using Winkler spring (beam-column) analysis techniques. The Weatherby analysis (Weatherby, Chung, Kim, and Briaud 1998) considered a simplified construction sequencing approach, identified in this report as the Shifted $R-y$ Curve method. This method is described in the following paragraph.

\subsection{Simplified Construction Sequencing Evaluation - Shifted $R-y$ Curve Method}

\subsubsection{Approximate modeling of the excavation sequence (based on para 3.3.1.2, Weatherby, Chung, Kim, and Briaud 1998)}

Construction activities may influence wall and soil behavior. Excavation stages, dewatering, installation and loading of the ground anchors, workmanship, and the time the excavation remains open can affect the behavior of the wall. Simulating the construction sequences for an anchored wall involves modeling a series of loading and unloading stages (Figure 6.14). 


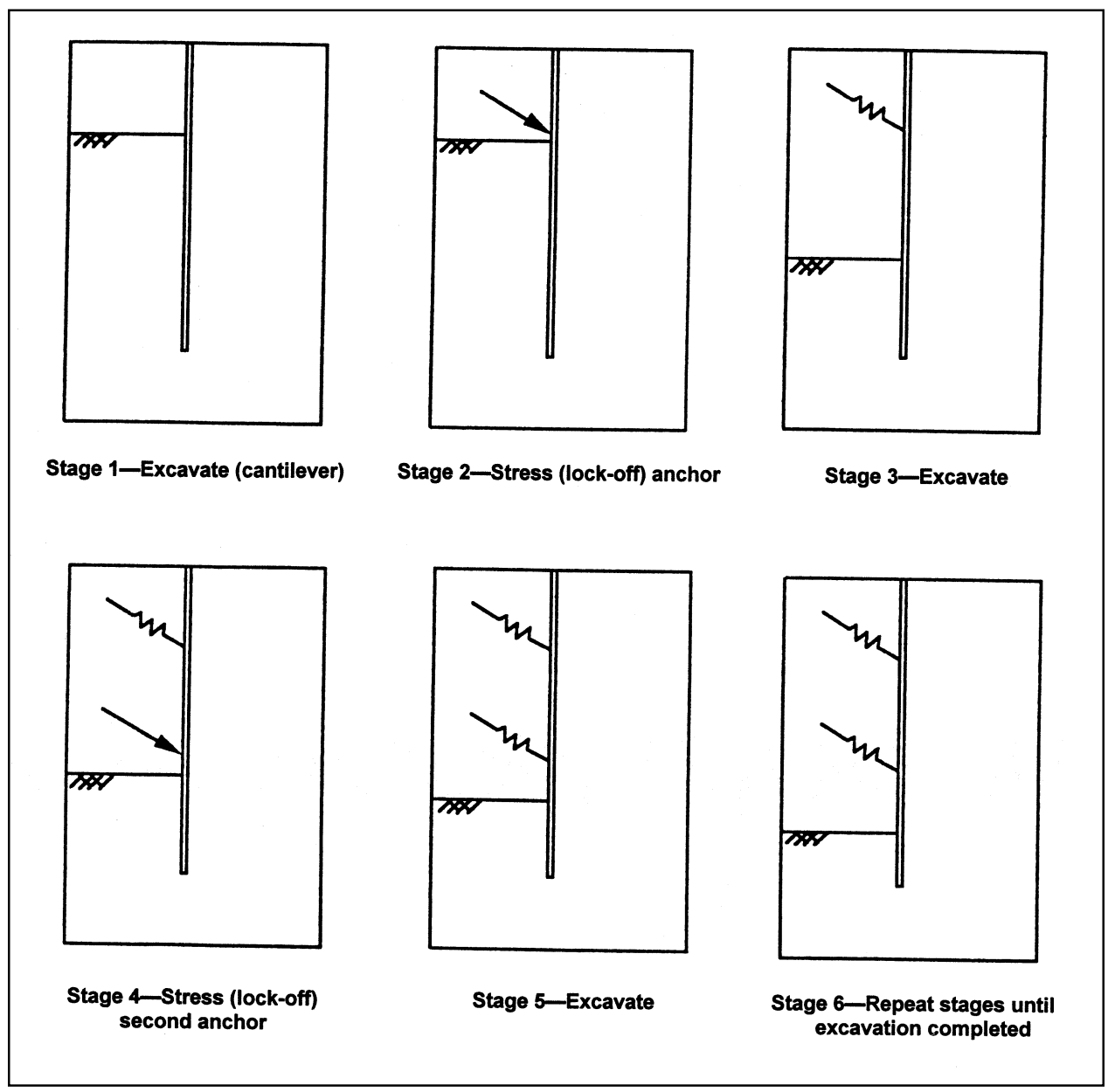

Figure 6.14. Typical construction stages in a soil-structure interaction analysis (after Figure 83; Weatherby, Chung, Kim, and Briaud 1998)

Surcharge loads, if applied, can be considered as a separate construction stage in the analysis. Ground retained behind the wall is unloaded as the wall deflects in response to excavating to the ground anchor level (cantilever stage). Deflections at some locations will be large enough to mobilize the active state of stress. Figure 6.15 shows idealized wall and soil response curves at three locations $\left(R_{1}, R_{2}\right.$, and $\left.R_{3}\right)$. In this report the wall and soil response curves are called $R-y$ curves to distinguish them from $P-y$ curves. At $R_{1}$, the wall moved laterally a distance represented by the deflection, $y_{1}$. This deflection is shown in the deflection profile for the wall and the $R-y$ curve for location $R_{1}$ (Figure 6.15). 


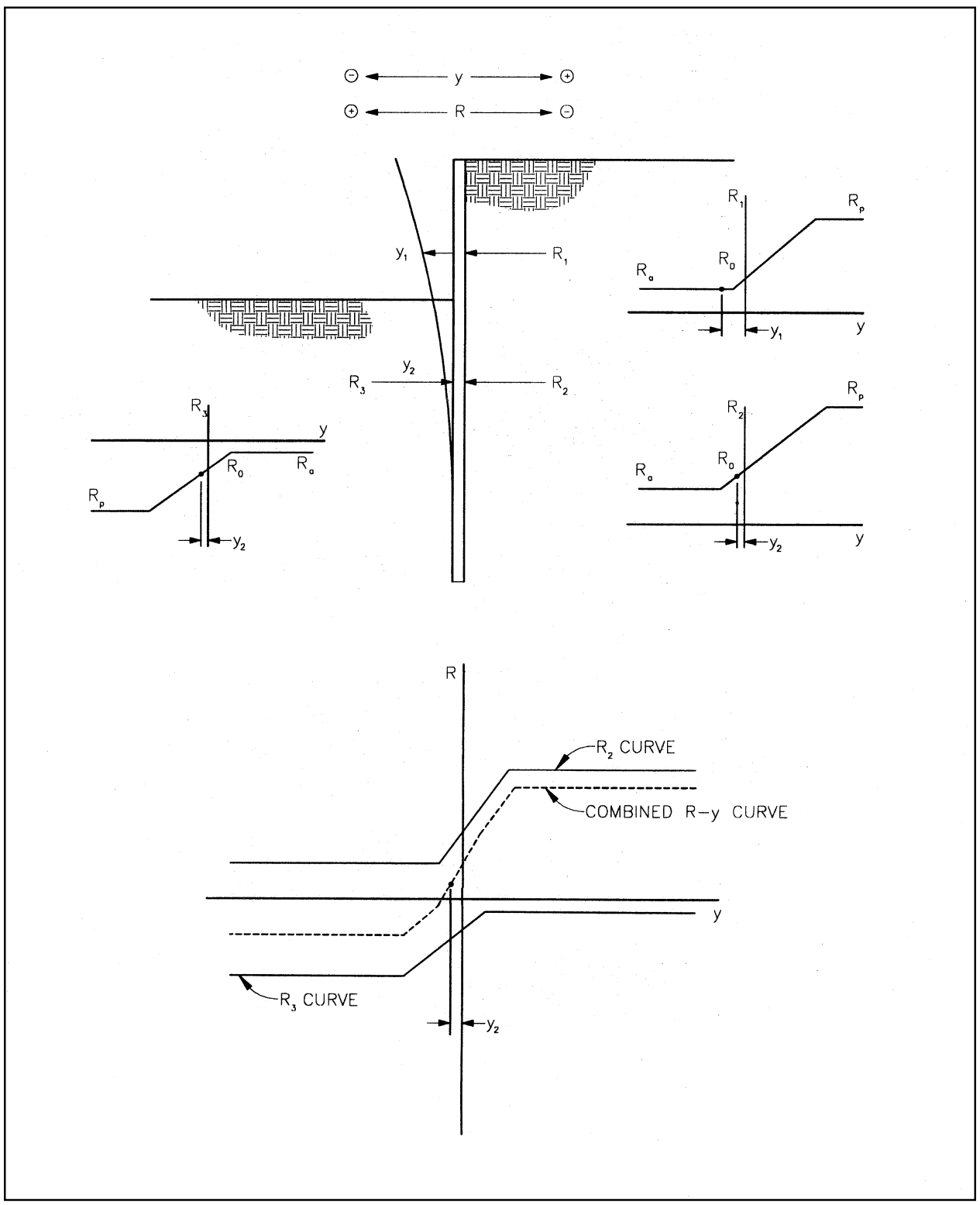

Figure 6.15. Diagram illustrating the $R-y$ curve for the cantilever stage (after Figure 84; Weatherby, Chung, Kim, and Briaud 1998)

The $R-y$ curve shows that the deflection exceeded the deflection necessary to mobilize the active state of stress (earth pressures remained constant with additional movement). Deflections greater than the reference deflections are plastic (nonrecoverable) movements. Below the bottom of the excavation, the wall moved outward to mobilized passive resistance. The response of the wall soil system below the bottom of the excavation for illustration purposes is represented by a combined $R-y$ curve, recognizing that, usually, for Winkler analyses separate $R-y$ curves are developed for each side of the wall. The combined $R-y$ curve is the sum of the $R_{2}$ and $R_{3}$ curves. 
When the ground anchor is stressed, the wall is pulled back into the ground and the soil is loaded by the wall. Figure 6.16 shows the idealized soil response curves for this stage of construction at the same locations as those shown in Figure 6.15 .

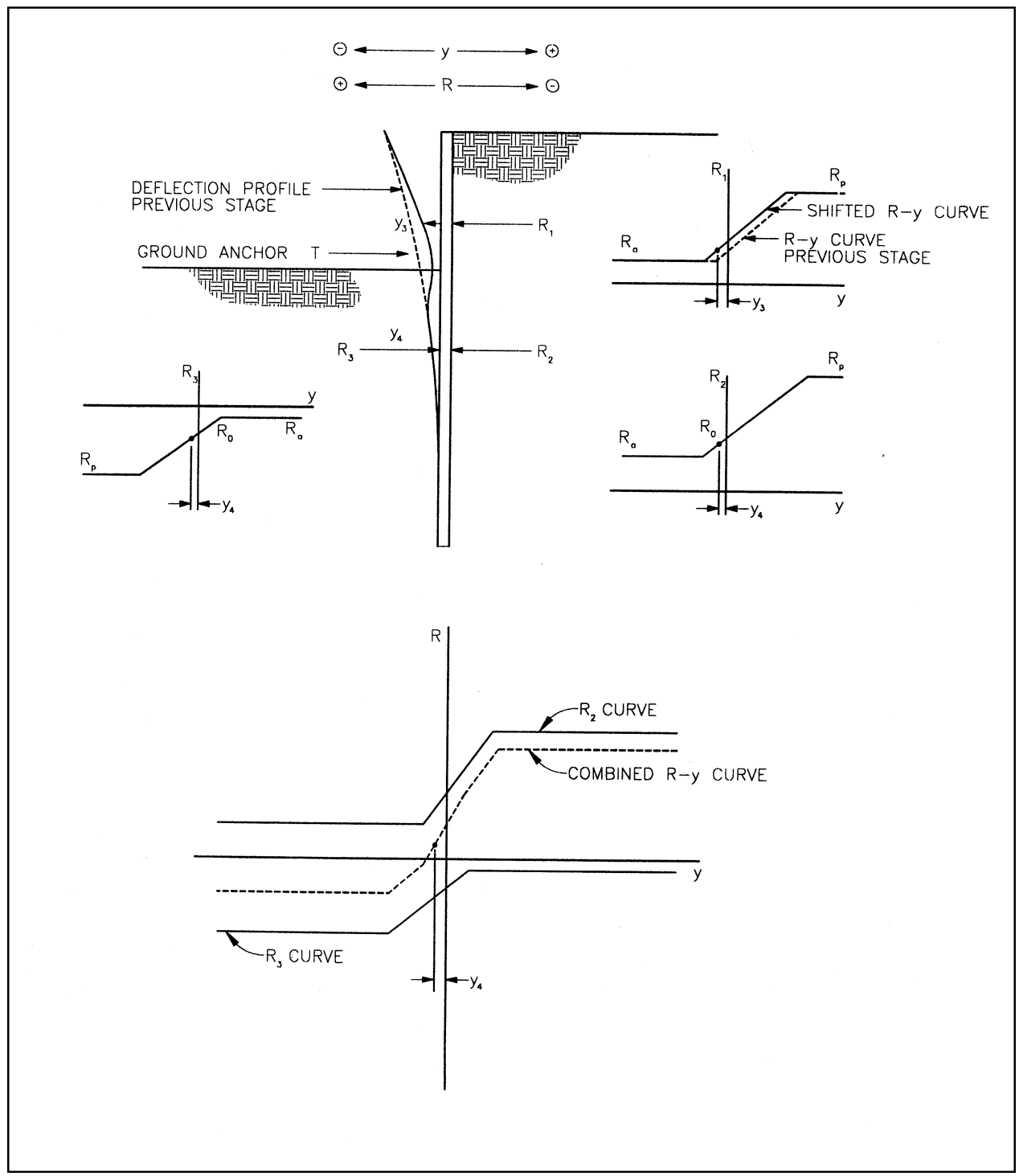

Figure 6.16. Diagram illustrating the $R$ - $y$ curve at anchor stressing (after Figure 85; Weatherby, Chung, Kim, and Briaud 1998)

At this stage of construction, the ground anchor is modeled as a concentrated load. The $\mathrm{R}_{1}$ curve in Figure 6.16 is shifted from the position shown in Figure 6.15 to account for the plastic movement that occurred at that location during the cantilever excavation stage. Figure 6.17 shows how the shifted $R_{1}$ curve was developed. In Figure 6.17 the $R-y$ curve for the cantilever stage (Curve 1) and the shifted $R-y$ curve for the anchor stressing stage (Curve 2) are shown. Curve 1 shows that, before any construction, the force on the wall was $R_{0}$ and the deflection was zero. As the cantilever excavation was made, the wall was unloaded. This step is represented by the cantilever excavation path in Figure 6.17. 


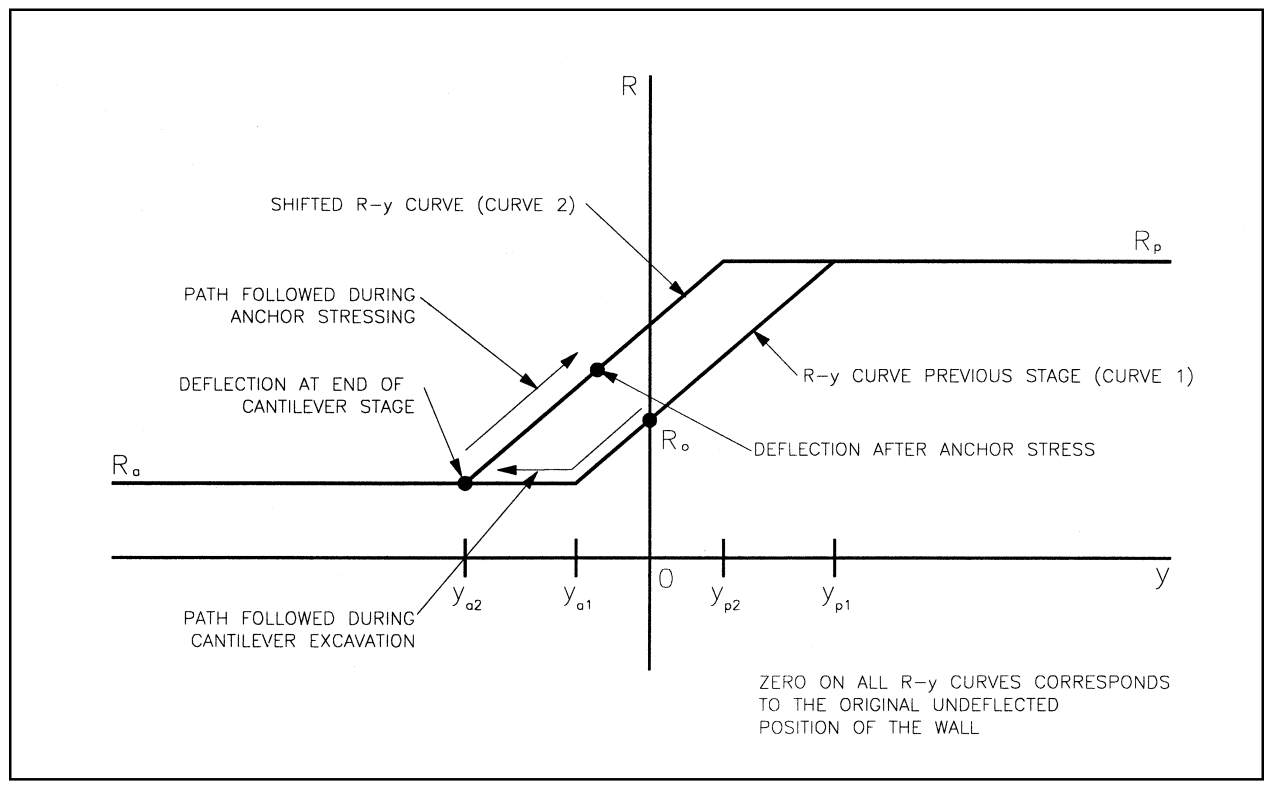

Figure 6.17. Shifted $R-y$ curve to model construction stages (after Figure 86; Weatherby, Chung, Kim, and Briaud 1998)

At the conclusion of the cantilever excavation, the force on the wall is $R_{a}$ and the deflection of the wall is $y_{a 2}$ (Figure 6.17), which is equal to $y_{1}$ (Figure 6.15). Since plastic movements occurred at $R_{1}$ during the cantilever stage, a new $R-y$ curve is required for the anchor stressing stage. The new $R-y$ curve is required because reloading associated with anchor stressing does not follow the unloading curve. Instead, passive resistance is mobilized when the wall moves back into the ground. The new $R-y$ curve (Curve 2) is developed by shifting Curve 1 an amount equal to the plastic movement from the previous stage $\left(y_{a 2}-y_{a 1}\right)$. The shifted $R-y$ curve (Curve 2) has new reference deflections $y_{a 2}$ and $y_{p 2}$, and it models the reloading response during anchor stressing. The anchor stress path is shown on Curve 2 in Figure 6.17. The $R_{2}$ and $R_{3}$ curves in Figure 6.15 were not shifted for the anchor stressing stage since the movements for the cantilever excavation and the stressing stage were in the elastic range.

Shifted $R$ - $y$ curves account for the effects of construction and allow beamcolumn methods to model anchored wall construction sequences without storing beam deflections from each construction stage. Shifted $R-y$ curves simulate construction activities by accumulating plastic movements from prior construction stages. When using the shifted $R-y$ curves in a beam-column program, the beam is assumed to be in its original position (zero deflection) for each run, and the shifted curves include the effects of construction activities. Anchor load, after lock-off, depends upon wall deflection at anchor location. A ground anchor is modeled by a load-deflection $(T-y)$ curve.

Figure 6.18 shows $R$ - $y$ curves and a $T-y$ curve for the ground anchor when the excavation extends below the ground anchor. The $R_{1}$ and $R_{2}$ curves from the anchor stressing (Figure 6.17) were not shifted since plastic movement did not occur during the anchor stressing stage. A new $R_{3}$ curve is shown in Figure 6.18 since the active and passive pressures on the left side of the wall changed in response to deepening the excavation. In developing the $T-y$ curve for the ground 
anchor, horizontal components of the ground anchor deflections and loads are used. The horizontal component of the anchor lock-off load corresponds to zero deflection of the wall after stressing. The initial slope of the $T-y$ curve is the anchor tendon stiffness. The $T-y$ curves change slope at the yield strength of the anchor tendon. The second portion of the curve represents the ground anchor behavior between yield and ultimate strength.

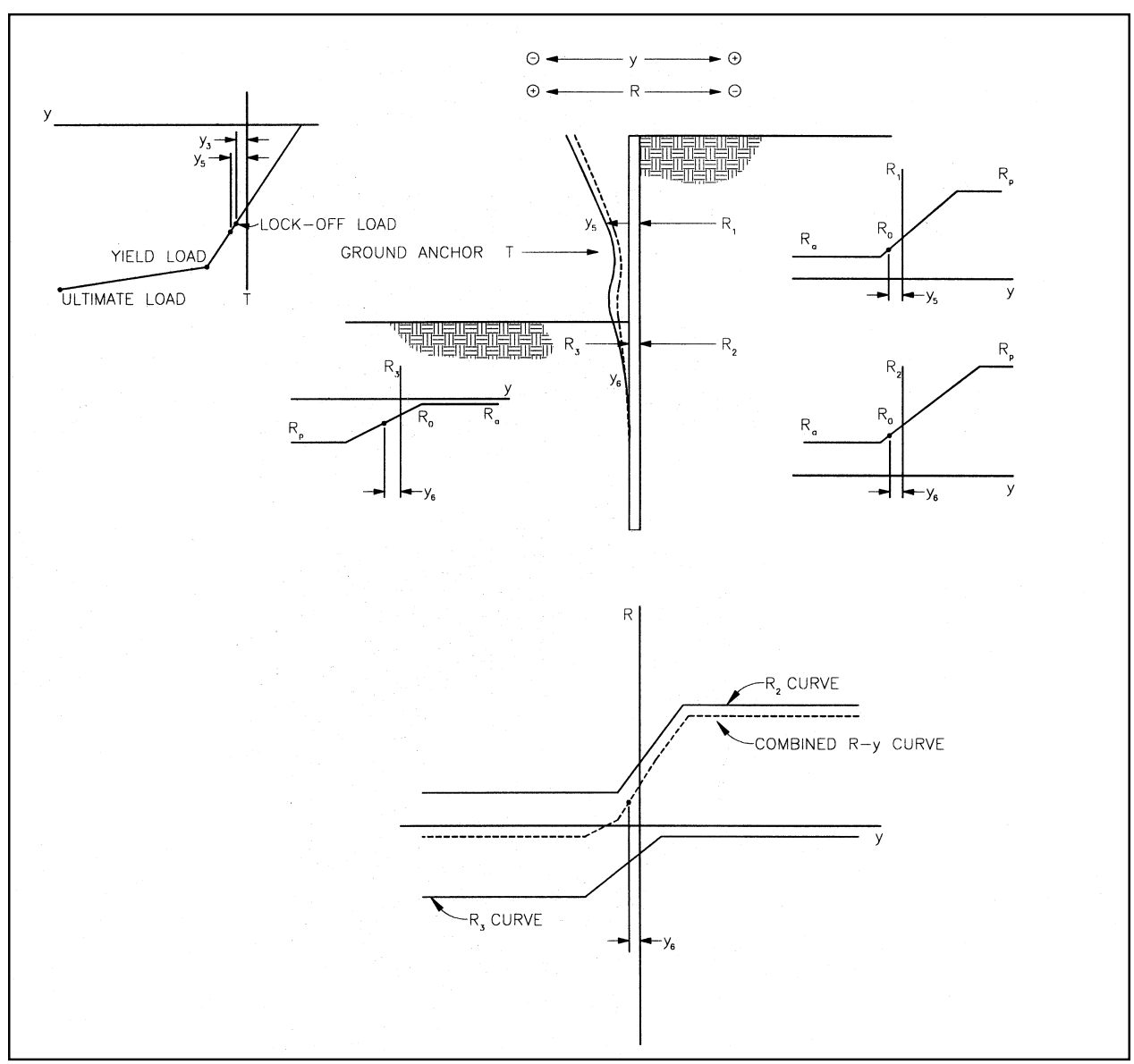

Figure 6.18. Diagram illustrating the $R-y$ curves during excavation below the ground anchor (after Figure 87; Weatherby, Chung, Kim, and Briaud 1998)

\subsubsection{Temporary tieback wall - Bonneville Navigation Lock}

Construction sequencing by Winkler spring analysis (Weatherby, Chung, Kim, and Briaud 1998) was used to evaluate the performance of the temporary tieback wall for the Bonneville Navigation Lock (see Figure 6.13). Soils at the site were assumed to be homogeneous sand, and plane strain $R-y$ curves for sand were used to determine nonlinear soil spring characteristics. Coulomb earth pressure coefficients were used to determine maximum (passive), minimum (active), and at-rest pressures for use in developing the $R-y$ curves. The reference deflection method was used to construct the $R-y$ curves. The reference deflections for the active and passive pressures were assumed to be $1.27 \mathrm{~mm}(0.05 \mathrm{in}$.) and $12.7 \mathrm{~mm}$ (0.5 in.), respectively. 
The construction sequence implemented in the Winkler spring analysis consisted of the following nine stages:

(1) Excavate to $1.8 \mathrm{~m}(6 \mathrm{ft})$ below ground surface.

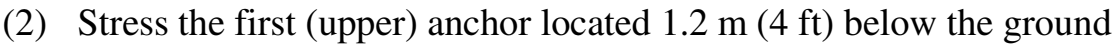
surface.

(3) Excavate to $5.5 \mathrm{~m}(18 \mathrm{ft})$ below ground surface.

(4) Stress the second anchor located $4.9 \mathrm{~m} \mathrm{(16} \mathrm{ft)} \mathrm{below} \mathrm{the} \mathrm{ground}$ surface.

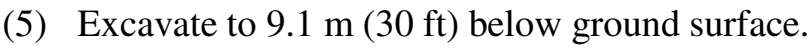

(6) Stress the third anchor located $8.5 \mathrm{~m}(28 \mathrm{ft})$ below the ground surface.

(7) Excavate to $12.2 \mathrm{~m}(40 \mathrm{ft})$ below ground surface.

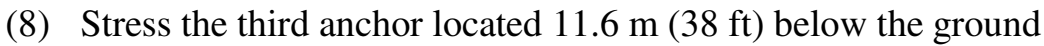
surface.

(9) Excavate to $12.8 \mathrm{~m}(42 \mathrm{ft})$ - completion of excavation stage.

Wall deflections, wall moments, and earth pressures predicted by the Winkler spring shifted $R-y$ curve construction sequence analysis (for the completion of excavation stage) are illustrated in Figure 6.19. Both measured and predicted deflection plots show that the wall was pulled back into the ground as the anchors were stressed. In the analysis, the $R-y$ curves were shifted where wall movements exceeded the reference deflections (deflections representing the break between elastic behavior and plastic behavior). This occurred for only the first stage of excavation prior to stressing the upper anchor. Below the upper anchor, movements were small and, therefore, the $R-y$ curves did not need to be shifted to model the construction sequence. Since most $R-y$ curves were not shifted, the predicted deflections for the analysis where the construction sequence was not modeled (Munger, Jones, and Johnson 1991) were similar to those for the analysis where the construction sequence was modeled.

The bending moments for the analysis where the construction sequence was not modeled were somewhat higher than those for the analysis where the construction sequence was modeled ( $75 \mathrm{ft}$-kips per foot versus $40 \mathrm{ft}$-kips per foot). The measured values for bending moment fell between the predicted values of the two analyses.

The NLFEM for the Bonneville temporary tieback wall is described in Chapter 7. In this particular instance, the Winkler spring analysis predicted the wall deflections satisfactorily. Accurate deflection predictions may have resulted from the large anchor loads, which prevented plastic movements from developing. Ground anchors with prestress load capacities high enough to pull the wall into the ground are sometimes necessary on Corps projects that are required to meet stringent displacement control performance objectives. Highcapacity ground anchors, such as those used at Bonneville, are not required for installations that are designed only to meet collapse-prevention performance objectives. The anchors used at Bonneville produced earth pressure loadings exceeding at-rest pressure conditions. 
As can be seen in Figure 6.19, the predicted earth pressures far exceed active earth pressures. They are also much greater than those represented by an apparent pressure diagram based on a total load equal to active pressures increased by a factor of safety of 1.3 applied to the shear strength of the soil. The maximum wall moment based on the apparent pressure diagram would be $12.7 \mathrm{ft}$-kips per foot $\left[(1 / 10)(1.050)(11)^{2}\right]$, which is about 10 percent of the actual wall maximum bending moment determined in the NLFEM analysis. The higher moments are due to the high earth pressures created by the tieback prestress loads (prestress loads that were required to limit wall displacements, to satisfy displacement control performance objectives).

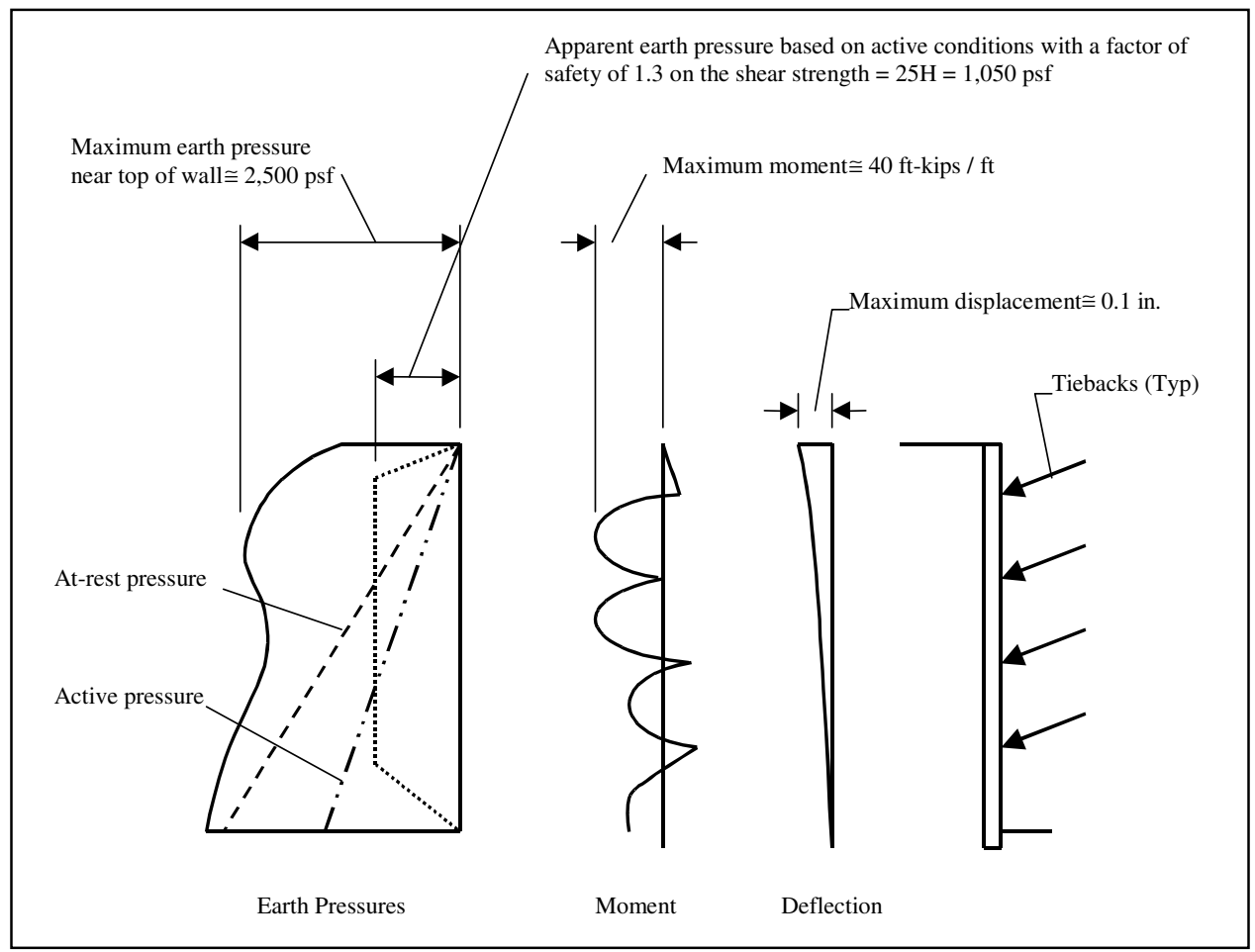

Figure 6.19. Winkler staged analysis - shifted $R$ - $y$ curve method (Bonneville Navigation Lock temporary tieback wall, final excavation results) (adapted from Figure 106; Weatherby, Chung, Kim, and Briaud 1998)

\subsection{Simplified Construction Sequencing Evaluation-Static Load Approach}

In the simplified approach, the soil pressure on the back side (driving side) of the wall is modeled as a static load, or following earth pressure force, equal to the design earth pressure. This procedure is described in Ratay (1996) and illustrated in ASCE/SEI (2000). For stiffer wall systems, the design earth pressure is something equal to, or approaching, at-rest pressure. The tiebacks are represented as spring supports and the resisting side soil as a series of Winkler soil springs that are preloaded to represent soil pressure conditions at each stage of the excavation. 
The wall displacement $(\delta)$ that occurs at a tieback location prior to installation of the tieback can be represented by a fictitious load, $k \delta$, applied against the reaction spring at that level or by imposing the $\delta$ displacement on that spring. Prestressing of the tieback can be modeled by applying an appropriate concentrated force at its location against the wall. The soil springs can be elastoplastic with the yield plateau set at the limiting passive pressure, or the soil springs can be linear-elastic and passive pressures monitored by the designer. In the latter case, any springs that exceeded the passive pressure limit would be removed, replaced by a passive pressure force, and the analysis rerun. This process would be repeated (iterative process) until all the Winkler springs were at or below the passive limit state. The iterative approach can be used with almost any structural analysis software and permits 3-D effects (plate action) to be evaluated. Three-dimensional effects are important with respect to behavior of slurry wall systems that are continuous.

This type of iterative approach was used to design the secant pile system for Monongahela River Locks and Dam 2. A 3-D analysis allowed the designer to evaluate the effects of load redistribution in cases where a single anchor failed. The simplified Winkler spring analysis for tieback wall systems is illustrated in Figure 6.20. When used to evaluate plate-bending effects in diaphragm walls and 3-D effects for a series of soldier beams or cylinder piles connected by a cap beam, this approach is also referred to in this report as a linear elastic finite element (LEFEM) analysis. This approach is demonstrated in ASCE/SEI (2000).

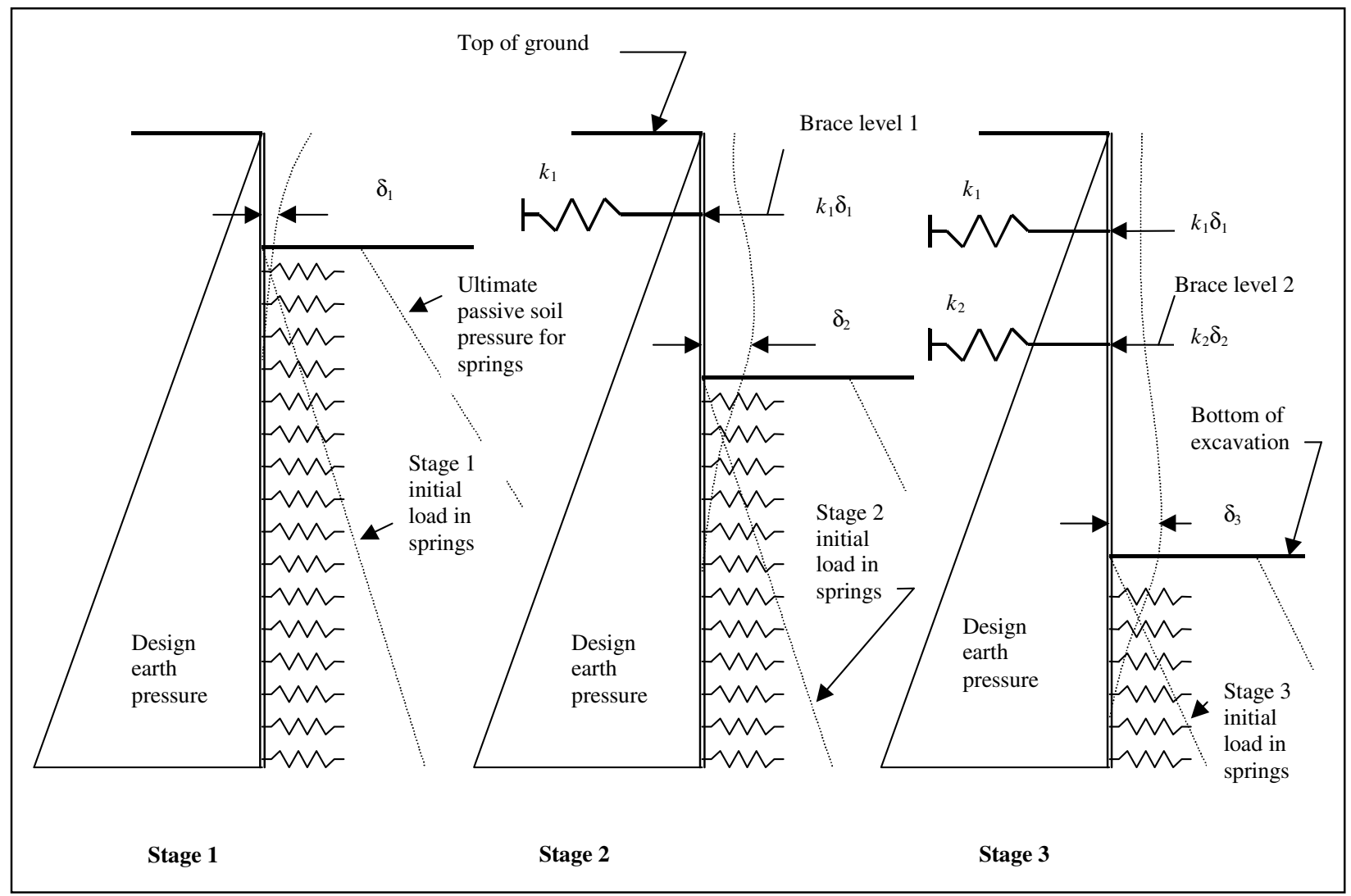

Figure 6.20. Simplified construction sequencing evaluation - static load approach 


\section{Finite Element Method (FEM) Analysis Procedure}

\subsection{Background}

Multi-anchored or tieback wall systems are often used for temporary support of excavations that have space restrictions due to adjacent structures, highways, railroads, etc. In some cases, multi-anchored systems may remain as permanent structures after construction. In Corps of Engineers projects, permanent tieback wall systems are used as guide walls and approach walls on navigation projects, and as retaining walls on highway and railroad protection and relocation projects.

The behavior of multi-anchored systems may be strongly influenced by factors such as the sequence of excavation and installation of anchors, fluctuations in the water table, and the nonlinear stress-strain behavior of soils. Therefore, to obtain accurate predictions of the magnitudes of stresses and deformations in the structure and the surrounding soil, it is necessary to perform soil-structure interaction (SSI) analyses that model the construction and operation stages of the system. For such analyses, adequate models for soils and soil-to-structure interfaces are required.

A substantial amount of research has been performed in recent years on another type of earth-retaining structure: navigational (such as lock walls). These studies have included SSI analyses of the Red River Lock and Dam No.1 (Ebeling, Mosher, Abraham, and Peters 1993; Ebeling and Mosher 1996; Ebeling, Peters, and Mosher 1997), the North Lock Wall at McAlpine Locks (Ebeling and Wahl 1997), and Locks 27 (Ebeling, Pace, and Morrison 1997) and are good examples of state-of-the-art techniques available for SSI analyses. These studies showed that the behavior of the soil-structure interface has a significant influence on the magnitudes of the loads acting against lock walls. They also illustrated that the pre- and post-construction stress paths followed by interface elements are complex, often involving simultaneous changes in normal and shear stresses, as well as unloading-reloading due to postconstruction rise of the groundwater level.

Gómez, Filz, and Ebeling (2000) developed an extended hyperbolic model for interfaces and implemented it into the finite element program SOILSTRUCTALPHA. The model is based on the Clough and Duncan (1971) hyperbolic formulation, which was extended to model a variety of stress paths. Gómez, Filz, and Ebeling (2000) performed a series of interface tests between uniform fine 
sands and concrete. Some of these tests followed complex stress paths that included unloading-reloading and simultaneous changes in normal and shear stresses.

Gómez, Filz, and Ebeling (2000) also carried out a pilot-scale lock wall simulation that modeled placement and compaction of the backfill, surcharge application, and changes in the elevation of the water table behind the wall. By comparing model predictions to interface test results, and the results of SOILSTRUCT-ALPHA analyses to measurements from the lock wall simulation, these investigators concluded that the extended load/unload/reload hyperbolic model might provide accurate estimates of the response of backfill-to-lock wall interfaces.

Important similarities exist between the types of loading that occur at structure-to-soil interfaces in both multi-anchored systems and lock walls. Therefore, it is possible that the Gómez-Filz-Ebeling model for interfaces could also be used for SSI analyses of multi-anchored systems. However, because the model was developed based on the results of interface tests performed using uniform fine sands, additional testing was required to validate model performance for coarser soils.

A series of virgin shear tests were performed under constant stress at the interface between a coarse sand and concrete. The results of these tests were used to determine the hyperbolic parameter values of the interface following the recommendations given by Gómez, Filz, and Ebeling (2000). An interface test was performed following a complex stress path that included unloading-reloading as well as simultaneous changes in shear and normal stresses. The interface response measured during this test was compared with the response calculated using the extended hyperbolic model. It was found that the Gómez-Filz-Ebeling interface model provided accurate estimates of the response of this type of interface. Therefore, it can be concluded that the extended hyperbolic model can be used for prediction of the response of interfaces between concrete and a variety of granular soils. The hyperbolic parameter values of the interface tested also add to the database of interface properties available in the literature. The extended hyperbolic model, together with the interface data that have been generated, provide useful tools for analyses of multi-anchored retaining systems and other U.S. Army Corps of Engineers structures.

\subsection{Common Types of Multi-Anchored Systems}

Multi-anchored systems can be constructed using different materials and configurations. The following are the most common types found in practice:

- Vertical sheet-pile systems with wales and post-tensioned tieback anchors.

- Soldier beam systems with wood or reinforced concrete lagging and posttensioned tieback anchors. 
- Secant cylinder pile systems with post-tensioned tieback anchors.

- Continuous reinforced concrete slurry wall systems with post-tensioned tieback anchors.

- Discrete concrete slurry wall systems (soldier beams with concrete lagging) with post-tensioned tieback anchors.

Figure 7.1 illustrates the use of a multi-anchored system for a typical navigation project. Because of the space restrictions imposed by an adjacent railroad, excavation for the expansion of the waterway requires the use of a multi-anchored system. For simplicity, it is assumed that the multi-anchored system depicted in the figure corresponds to a continuous, reinforced concrete slurry wall with tieback anchors. Tiebacks consist of post-tensioned tendons with a grouted anchor region. A berm of granular material or riprap is placed at the toe of the wall to minimize erosion and improve stability.

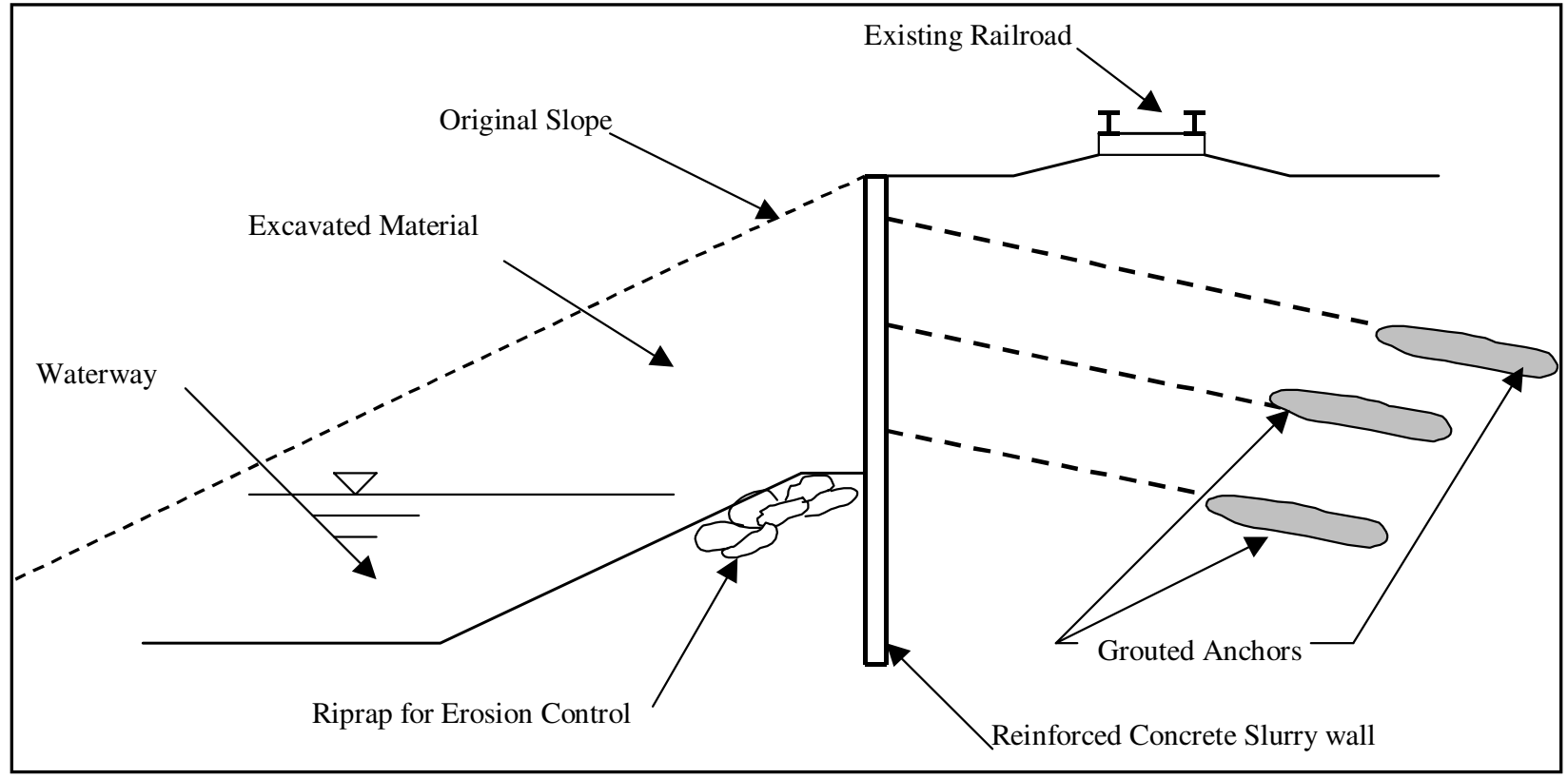

Figure 7.1. Typical multi-anchored tieback wall system for Corps navigation project

Figure 7.2 illustrates the typical construction sequence of a reinforced concrete slurry wall. Initially, a trench is excavated using a clamshell-type tool. The excavation is stabilized by the use of mud slurry. The finished trench acts as formwork for the reinforced concrete panel. Placement of the concrete using a tremie pipe displaces the mud slurry and leaves a structural concrete wall that can be excavated and tied back in much the same manner as the other tieback wall systems. The walls are reinforced using preassembled cages, which are dropped into the slurry trench just before concrete placement. Slurry wall systems are usually 0.6 to $0.9 \mathrm{~m}$ ( 2 to $3 \mathrm{ft}$ ) thick and can be placed to depths of $30 \mathrm{~m}(100 \mathrm{ft})$ or more. The construction process can be summarized as follows: 


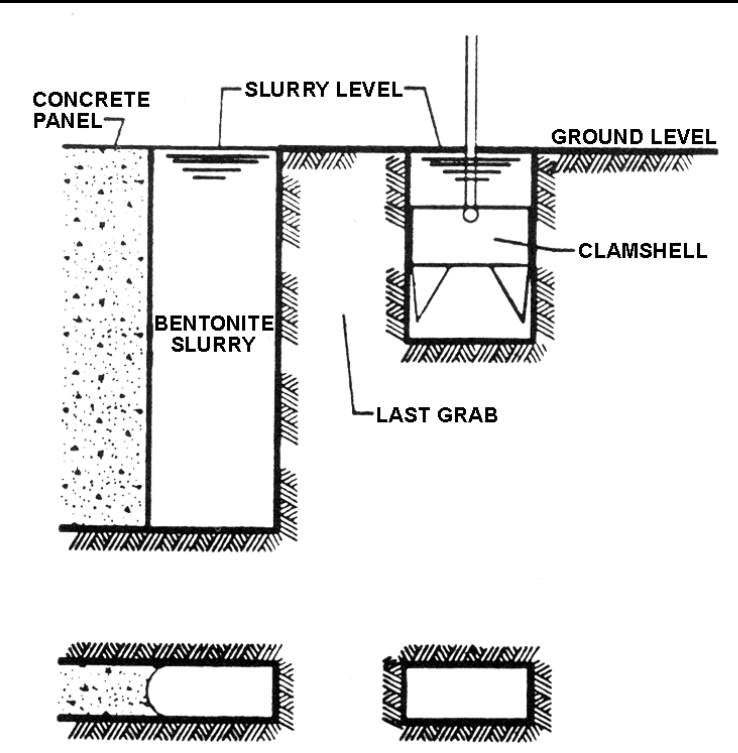

a. Excavation under mud slurry

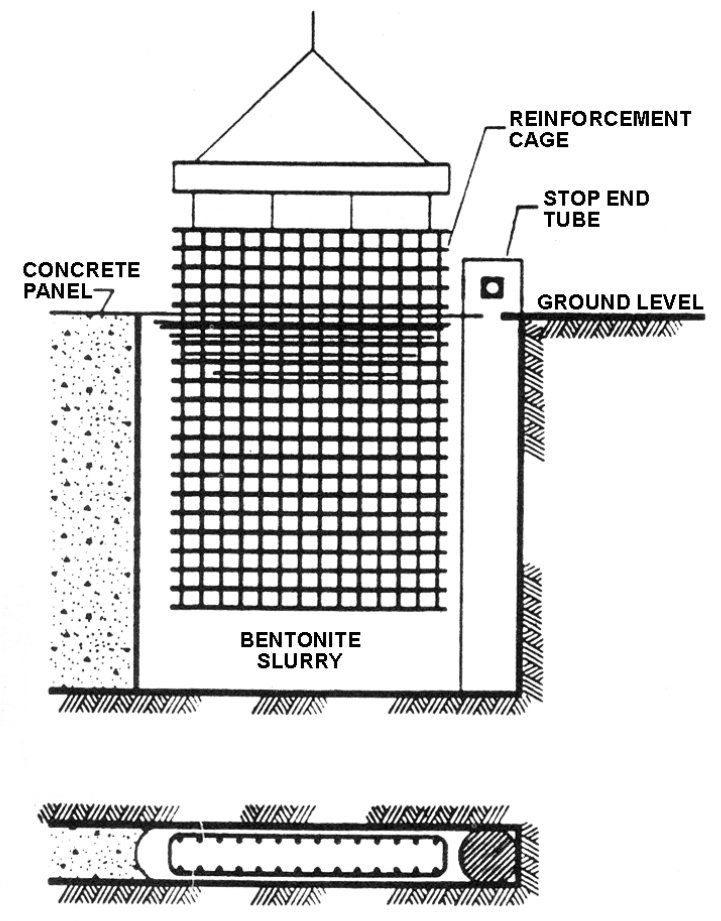

\section{c. Placement of reinforcement cage}
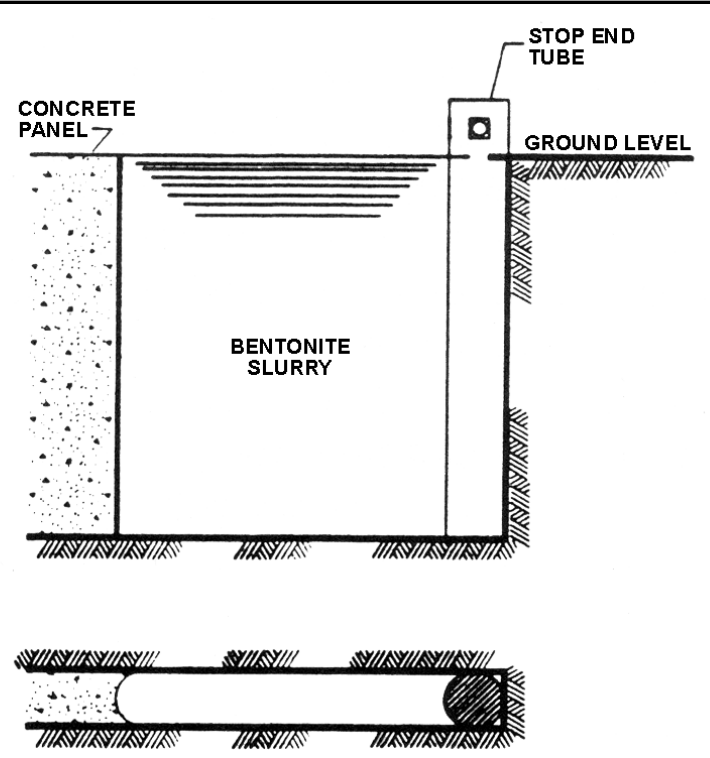

b. Placement of stop end tube

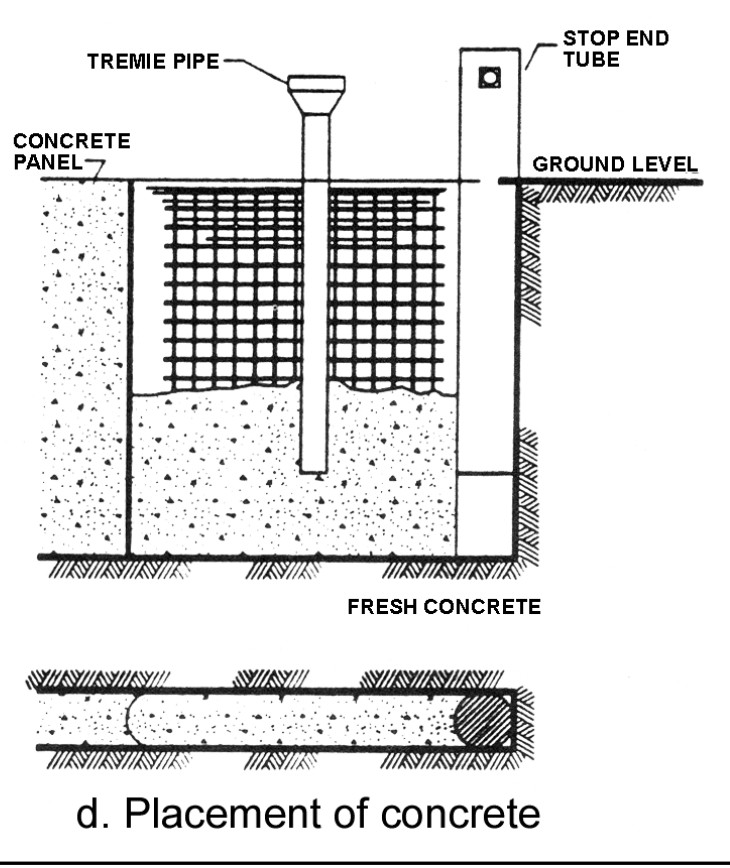

Figure 7.2. Typical construction sequence of a reinforced concrete slurry wall

a. Guide walls are constructed to facilitate positioning and alignment of the clamshell during the excavation process. To stabilize the excavation, mud slurry is kept inside the excavation to a level above the water table. As illustrated in Figure 7.2a, the excavation for each panel follows a 
staggered sequence. Two end excavations are performed first, leaving a central core intact. After the end excavations are completed, the central core is removed.

$b$. A stop end tube is placed at one end of the panel excavation. This tube is extracted after concrete placement, leaving a semicircular indentation.

This indentation serves as a guide for the excavation of the adjacent panel and allows the creation of a shear key between the panels.

c. Once the panel has been excavated to the desired depth and the slurry cleaned of fine excavation material (desanded), the reinforcement cage is lowered into the excavation.

d. One or more tremie pipes are used to place the concrete without contamination from the slurry.

$e$. Once the wall is finished and the concrete reaches its desired strength, the excavation and tieback installation process can begin.

Construction of the second navigation lock at Bonneville Lock and Dam required the use of concrete slurry walls to retain the foundation of an adjacent railroad line. Detailed descriptions of construction procedures for the continuous reinforced concrete slurry wall and for the discrete slurry wall systems used at Bonneville Lock are presented by Munger, Jones, and Johnson (1991) and Maurseth and Sedey (1991), respectively.

\subsection{Response of Soil-to-Wall Interfaces in Multi- Anchored Systems}

A waterways expansion project, such as that presented in Figure 7.1, requires performing SSI analyses to determine the magnitude of the deformations of the soil above the excavation, and the bending moments and stresses in the retaining wall. Such analyses require close modeling of the construction stages of the multianchored system, as well as adequate constitutive models for the soil and for the interfaces between soil and structural components. The finite element analyses performed by Mosher and Knowles (1990) for the tieback walls at Bonneville Lock and Dam are a good example of the available techniques that can be used in SSI analyses of multi-anchored systems.

Figure 7.3 illustrates some of the construction and operation stages of the hypothetical navigation project shown in Figure 7.1. For simplicity, it is assumed that construction is performed in the dry. After completion of the continuous reinforced concrete slurry wall (Figure 7.3b), the soil in front of the wall is excavated to an elevation slightly below the position of the first row of anchors. The anchors are then installed and tensioned according to the project specifications (Figure 7.3c). Once these anchors are tensioned and tested, excavation continues until reaching the position of the second row of anchors. The process is repeated until reaching the bottom of the excavation (Figures $7.3 \mathrm{~d}$ and 7.3e). Once the excavation is completed, the granular toe berm is placed against 


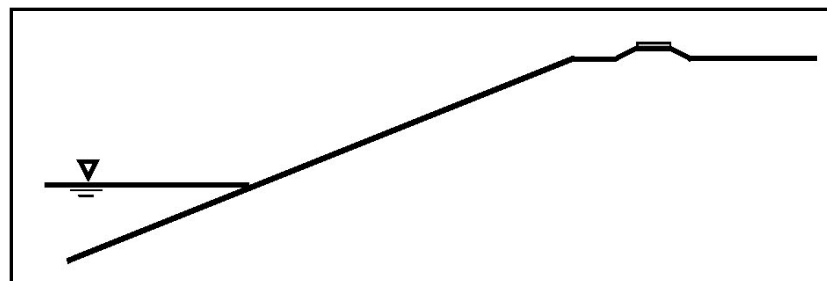

a. Initial state

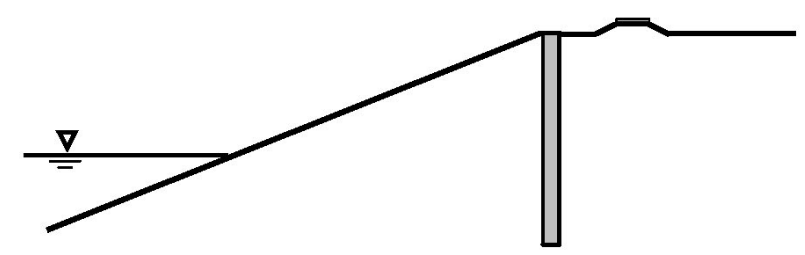

b. Construction of reinforced concrete slurry wall

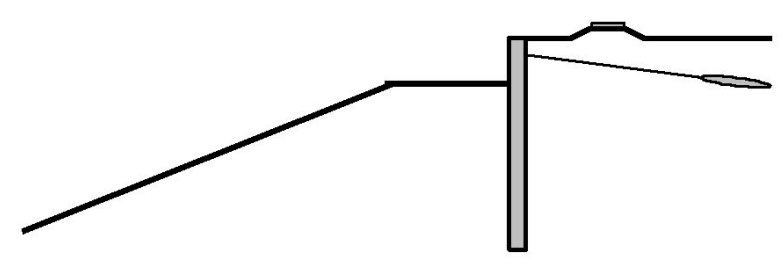

c. First stage of excavation and installation of anchors

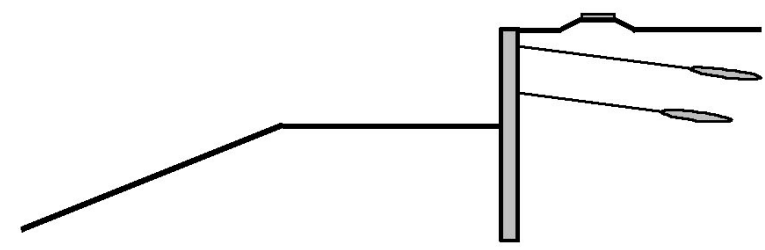

d. Intermediate stage of excavation and installation of anchors

Figure 7.3. Typical construction and operation stages

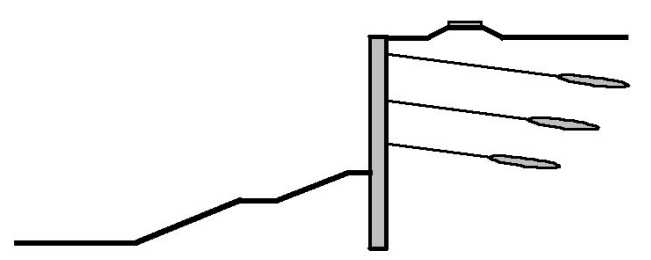

e. Final excavation and installation of bottom row of anchors

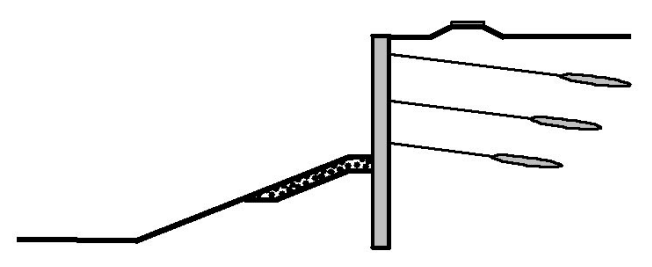

f. Construction of riprap blanket

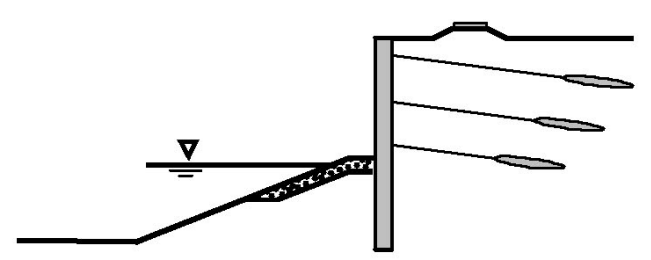

g. Operational stage 
the toe of the wall (Figure 7.3f). During operation of the navigation facility, the water level outside the wall reaches its normal elevation, which may fluctuate periodically during the life of the structure (Figure $7.3 \mathrm{~g}$ ).

Figure 7.4 illustrates the type of loading expected to occur on a soil-to-wall interface element during construction of such multi-anchored systems. Immediately after construction of the slurry wall, the interface element is subjected to a normal stress $\sigma_{n}$ (Figure 7.4a). Because little or no relative movement has taken place between the soil and the wall, the shear stress acting on the interface element at this stage may be assumed zero. Excavation of the soil in front of the structure may induce outward deformations of the wall and reduction in the lateral stresses within the soil mass behind the wall. The relative settlement of the soil behind the wall, which may take place as a consequence of the reduction in lateral stresses, induces shear on the soil-to-wall interface element (Figure 7.4b). Subsequent installation and tensioning of a row of anchors may increase the normal stresses acting on the interface element. Tensioning of anchors may also induce relative heave of the soil mass behind the wall and a consequent reduction in the shear stress acting on the interface (Figure 7.4c).

Subsequent stages of excavation and installation of anchors may produce progressive shearing of the interface element under varying normal stress with intermediate cycles of unloading-reloading. During the life of the structure, fluctuations of the water level on both sides of the wall may induce further cycles of unloading and reloading of the wall-soil interface.

The type of loading imposed on the soil-to-wall interface of a multi-anchored system may differ from the simplified loading mechanisms illustrated in Figure 7.4. Factors such as the sequence of excavation, the distribution of anchors, the stiffness of the wall, and the response of the foundation soil will influence the behavior of the soil-wall system. 


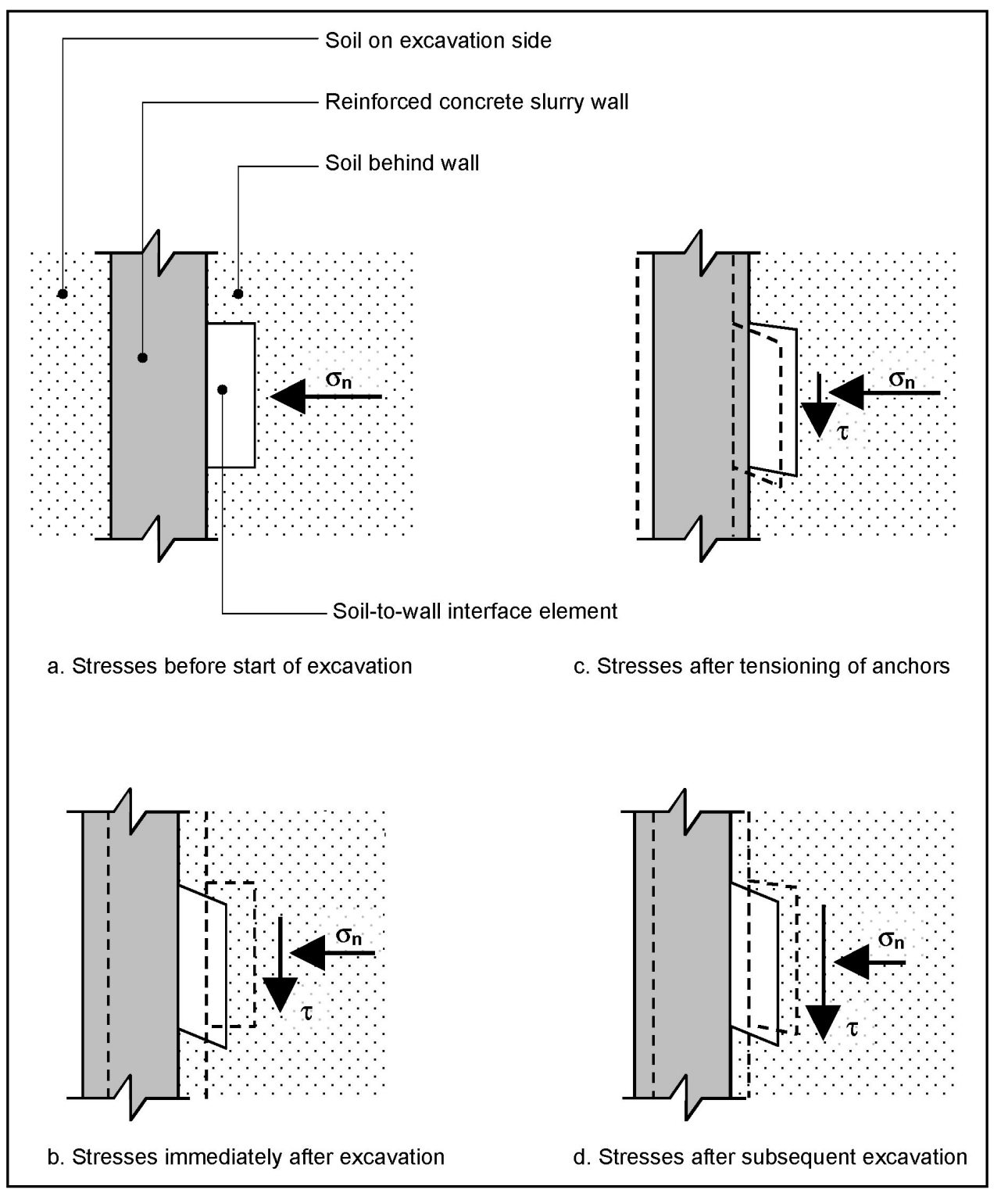

Figure 7.4. Loading on soil-to-wall interface

\subsection{Gómez-Filz-Ebeling Interface Model}

A number of interface constitutive models have been developed by different authors. Quasi-linear models have been used by Goodman, Taylor, and Brekke (1968); Desai, Muqtadir, and Scheele (1986); Matsui and San (1979); and Wong, Kulhawy, and Ingraffea (1989). Nonlinear models have been used by Clough and Duncan (1971); Zaman, Desai, and Drumm (1984); Desai, Drumm and Zaman (1985); and others.

Clough and Duncan (1971) developed the hyperbolic model for interfaces. This model has been used extensively in SSI analyses and design of geotechnical structures, including analyses of lock wall behavior (Ebeling, Mosher, Abraham, 
and Peters 1993; Ebeling and Mosher 1996; Ebeling, Peters, and Mosher 1997; Ebeling and Wahl 1997; and Ebeling, Pace, and Morrison 1997). The hyperbolic model can provide an accurate approximation of the interface response under monotonic loading at constant normal stress. A recent study by Gómez, Filz, and Ebeling (2000) has shown that the original Clough and Duncan (1971) interface model is not accurate for modeling the interface response under simultaneous changes in shear and normal stresses or for unloading-reloading.

Gómez, Filz, and Ebeling (2000) introduced the extended hyperbolic model for interfaces and implemented it into the finite element program SOILSTRUCTALPHA. The model is based on the Clough and Duncan (1971) hyperbolic formulation and incorporates new features to model a variety of stress paths. The Gómez-Filz-Ebeling model does not require any interface parameter values in addition to those used in the Clough and Duncan (1971) hyperbolic model. Therefore, hyperbolic parameter values available in the literature (Clough and Duncan 1969; Petersen, Kulhway, Nucci, and Wasil 1976; Lee, Kane, Drumm, and Bennett 1989) for a variety of interfaces can be used with the Gómez-FilzEbeling model.

Gómez, Filz, and Ebeling (2000) performed a series of interface tests between uniform, fine sands and concrete. Some of their tests followed complicated stress paths that included unloading-reloading and simultaneous changes in normal and shear stresses. Comparisons between test results and model predictions showed that the extended hyperbolic model could accurately predict the response of interfaces of the types tested.

Gómez, Filz, and Ebeling (2000) also carried out a pilot-scale lock wall simulation that modeled placement and compaction of backfill, surcharge application, and changes in the elevation of the water table behind the wall. The backfill consisted of a uniform fine sand identical to one of the soils used for interface testing. The lock wall simulation test was also modeled using SOILSTRUCT-ALPHA. Comparison between the data from the lock wall simulation and the results of the SOILSTRUCT-ALPHA analyses confirmed the accuracy of the extended hyperbolic model and its applicability for SSI analyses of lock walls.

The Gómez-Filz-Ebeling model is also presumed to be applicable for SSI analyses of multi-anchored systems. As illustrated by the simplified interface loading mechanism in Figure 7.4, the soil-to-wall interface in a reinforced concrete slurry wall may be subject to simultaneous changes in shear and normal stresses, as well as unloading-reloading. The Gómez-Filz-Ebeling model is accurate for predicting the response of interfaces to this type of loading. While the model has been verified against the results of tests performed on interfaces between fine sands and concrete, it has not been evaluated against results of tests performed on interfaces between coarse sands and concrete. In addition, further work on interface testing is required to expand the existing database on interface hyperbolic parameter values.

It was found that the Gómez-Filz-Ebeling model provides accurate estimates for the coarse sand-to-concrete interface. It can be concluded from this result and previous work that the Gómez-Filz-Ebeling model provides accurate predictions 
of the response of interfaces between concrete and a variety of granular soils. The hyperbolic parameters for the coarse sand-to-concrete interface, determined from the tests, also add to the database of interface parameter values.

\subsection{Need for Nonlinear Finite Element SSI Analyses}

The RIGID method described in Chapter 5 does not take soil deformations into account and, although widely used, has limited application for those circumstances in which actual load-displacement characteristics of the system are desired. The Winkler method described in Chapter 6 relates soil pressures to wall deformations and, for that reason, can be considered an improved method of analysis, especially when used in a staged construction analysis. However, it has not been a reliable tool for evaluating soil displacements that occur either in the soil mass or at the ground surface of the soil retained by the tieback wall. Wall displacement information provided by the Winkler analysis is unreliable because the value of the coefficient of horizontal subgrade reaction is dependent on the extent of the zone of influence, a quantity that is difficult to properly establish. In addition, arching that takes place in the retained soil influences the soil displacement-pressure response.

The nonlinear finite element soil structure interaction analysis method (NLFEM) described above can overcome the shortcomings of the other analysis procedures since it permits a complete and precise solution based on the stressstrain laws for the soils involved, the boundary conditions of the problem, and the basic equations of mechanics. Although the finite element method is the most suitable one, practical difficulties are such that it is not routinely used in the design or evaluation of tieback wall systems.

As indicated above, the NLFEM has been used with success to evaluate the performance of tieback wall systems. Its particular application with respect to the performance evaluation of a temporary tieback wall used to facilitate construction of the Bonneville Navigation Lock (Mosher and Knowles 1990) is described below. The NLFEM provided a detailed and accurate representation of the tieback wall-soil system response to various loadings that occurred prior to, during, and after wall construction. The objectives of the NLFEM were to confirm by incremental analysis that ground movements behind the wall during and after construction would meet stringent displacement requirements. The results of the analysis were confirmed by instrumentation. The Bonneville Navigation Lock NLFEM analysis is described in detail to demonstrate the differences between actual behavior and that usually assumed in the design of tieback wall systems. These differences are especially noticeable with respect to stiff wall systems with high anchor prestress loads. 


\subsection{Bonneville Temporary Tieback Wall Analysis}

\subsubsection{Wall description}

The temporary tieback retaining wall is approximately $134 \mathrm{~m}(440 \mathrm{ft})$ long. The wall is constructed by slurry trench methods in $6-\mathrm{m}(20-\mathrm{ft})$-long sections similar to those shown in the section view (horizontal) of Figure 7.5.

The heights of the panels ranged from 6 to $34 \mathrm{~m}$ (20 to $110 \mathrm{ft})$. Excavation sequencing was similar to that shown in Figure 7.3. Anchors were installed in a grid pattern of approximately $3 \mathrm{~m}(10 \mathrm{ft})$ horizontal by $3.4 \mathrm{~m}(11 \mathrm{ft})$ vertical. Each tieback anchor was composed of nineteen 15-mm (0.6-in.)-diam strands with a guaranteed ultimate strength of $1,862 \mathrm{mPa}(270 \mathrm{ksi})$. Each anchor was prestressed to 150 percent of its design load. The design loads are approximately 50 percent of the anchor ultimate load capacity. Panel 6 was the focus of the NLFEM analysis. A section view (vertical) of Panel 6 is shown as Figure 7.6. The tieback anchor loads are summarized in Table 7.1.

\subsubsection{Overall design and evaluation process}

The overall design and evaluation process for the Bonneville Navigation Lock temporary tieback wall involved a RIGID analysis and a Winkler analysis, in addition to the nonlinear soil structure finite element analysis described in this chapter. The purposes and results of the RIGID and Winkler analyses are described in general terms and illustrated using Figure 7.7.

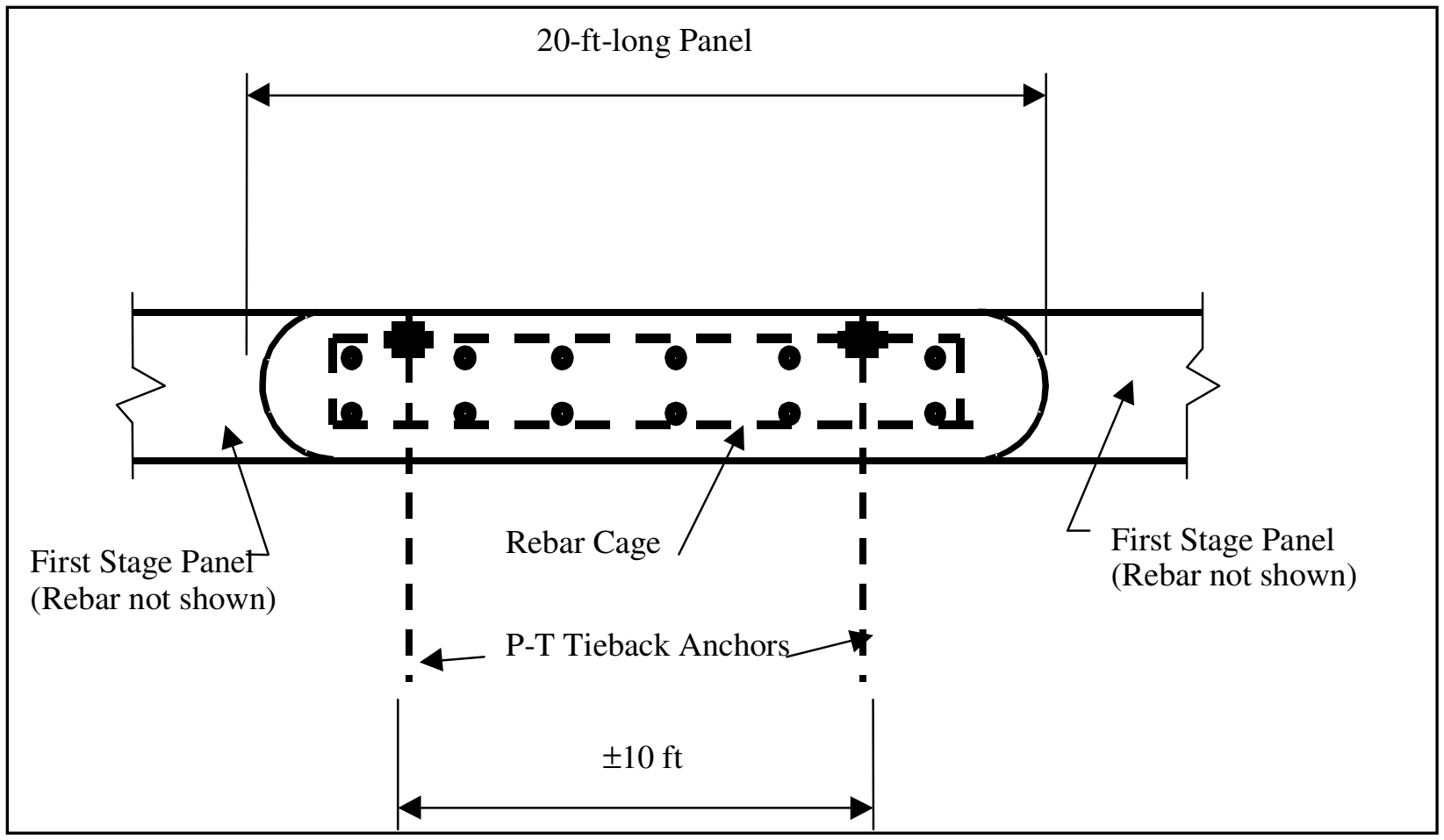

Figure 7.5. Bonneville Navigation Lock, temporary tieback wall-horizontal section 


\subsubsection{RIGID analysis}

A simple preliminary analysis using the RIGID analysis procedure was required to estimate the size and spacing of anchors. The RIGID analysis for Bonneville was based on a composite apparent pressure diagram as shown in Figure 7.7c. The upper rectangular-shaped region is based on at-rest pressures (in accordance with Figure 29, Chapter 3, of Design Manual 7.2 (NAVFAC 1982). The lower triangular-shaped region is also based on at-rest pressures (Coulomb's equation with a factor of safety of 1.5 applied to the shear strength of the soil). This composite diagram assumed that wall displacements would be small, thereby keeping earth pressure near an at-rest state. The triangular lower portion of the diagram was considered to be appropriate since the upper anchors could possibly lose some tension during the life of the wall, thereby leading to higher loads at the base of the wall. Anchors sizes were estimated based on a continuous beam analysis using the pressure diagram loading of Figure 7.7c. There was very little difference between the anchor loads obtained from the continuous beam analysis and those determined using the tributary area method (Munger, Jones, and Johnson 1991).

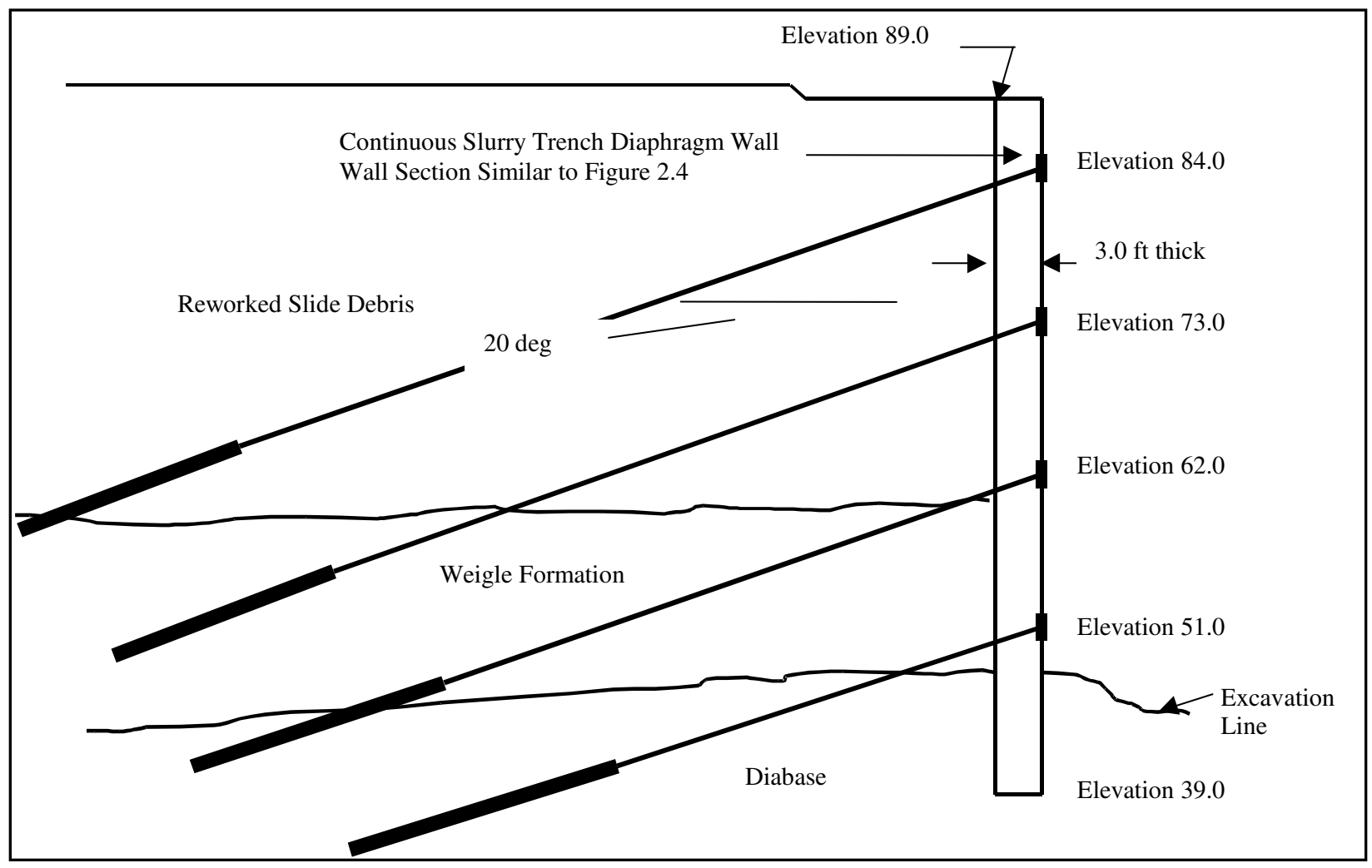

Figure 7.6. Bonneville Navigation Lock, temporary tieback wall-vertical section 


\begin{tabular}{|c|c|c|c|c|c|c|c|c|c|}
\hline \multicolumn{10}{|c|}{$\begin{array}{l}\text { Table } 7.1 \\
\text { Panel } 6 \text { Anchor Loads }\end{array}$} \\
\hline \multicolumn{2}{|c|}{ Anchor Elevation } & \multicolumn{2}{|c|}{ Anchor Length } & \multicolumn{2}{|c|}{ Design Load } & \multicolumn{2}{|c|}{$\begin{array}{c}\text { Prestress Load } \\
\text { @ } 150 \text { percent } \\
\text { Design Load }\end{array}$} & \multicolumn{2}{|c|}{ Lock-Off Load } \\
\hline $\mathbf{m}$ & $\mathrm{ft}$ & $\mathbf{m}$ & $\mathrm{ft}$ & $\mathbf{k N}$ & kips & $\mathbf{k N}$ & kips & $\mathbf{k N}$ & kips \\
\hline 25.6 & 84 & 27.1 & 89 & $1,249.9$ & 281 & $1,874.9$ & 421.5 & $1,209.9$ & 272 \\
\hline 22.3 & 73 & 24.1 & 79 & $1,249.9$ & 281 & $1,874.9$ & 421.5 & $1,298.8$ & 292 \\
\hline 18.9 & 62 & 20.7 & 68 & $1,249.9$ & 281 & $1,874.9$ & 421.5 & $1,289.9$ & 290 \\
\hline 15.5 & 51 & 15.8 & 52 & $1,592.5$ & 358 & $2,388.7$ & 537.0 & $1,583.6$ & 356 \\
\hline
\end{tabular}

The wall bending moment diagram resulting from a RIGID analysis is illustrated in Figure 7.7b. Because this type of analysis does not consider the wall displacement that occurs at anchor locations, it will underestimate actual bending moment demands on the wall. Therefore, a Winkler analysis was performed to estimate wall bending moments and to confirm anchor loads.

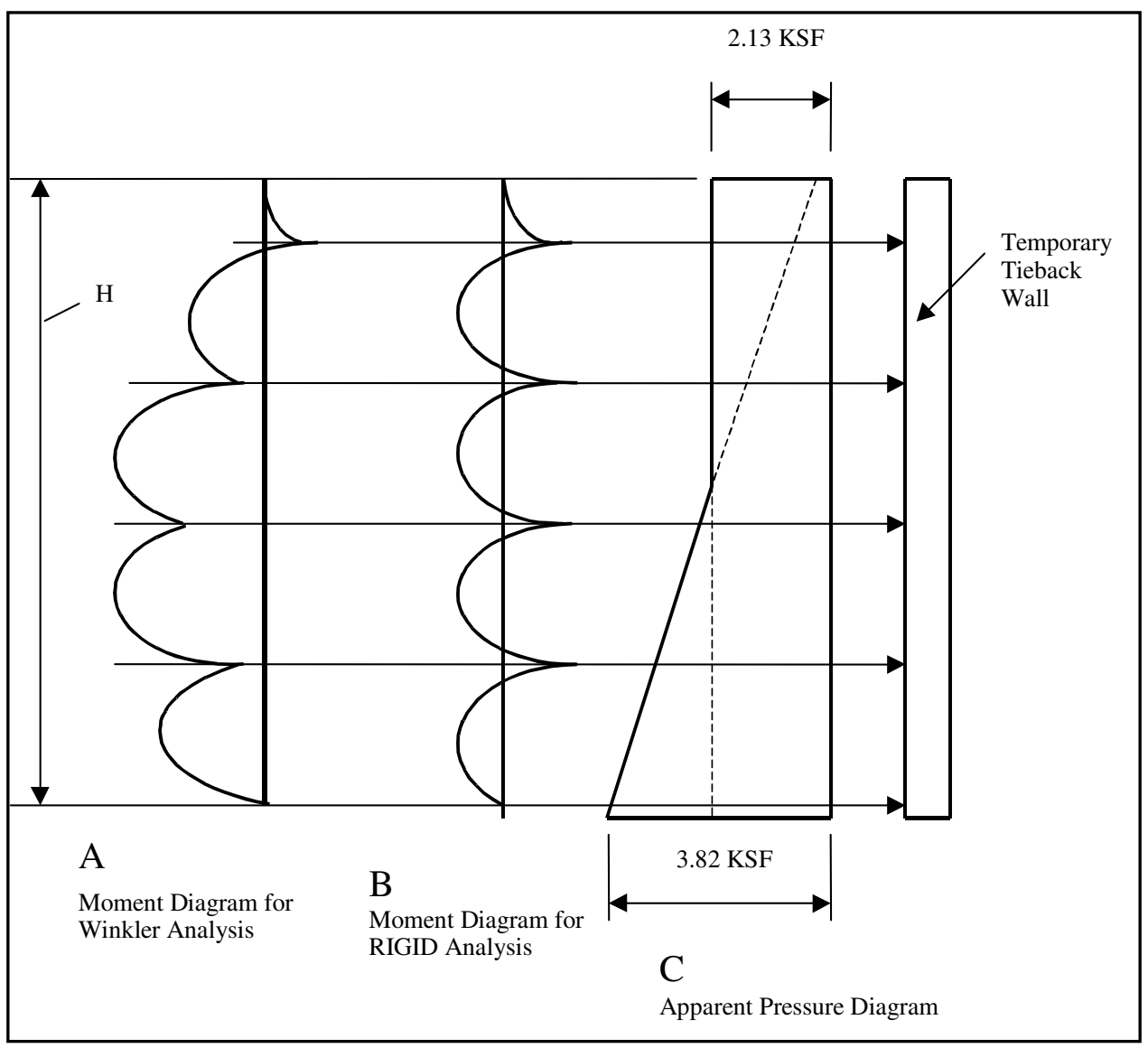

Figure 7.7. Overall design and evaluation process 


\subsubsection{Winkler analysis}

The Winkler analysis used to evaluate the Bonneville Navigation Lock temporary tieback wall is described in Chapter 6 of this report and in Munger, Jones, and Johnson (1991). The moment diagram for the wall was determined using a one-step analysis that modeled the final excavation condition only. The moment diagram for the Winkler analysis is illustrated in Figure 7.7a. This analysis, because it accounts for wall displacements at anchor locations, does a good job of estimating moment demands on the wall. A RIGID analysis that considers construction staging and considers support displacements that accumulate during each stage of construction (yielding supports analysis) could also provide good results.

As illustrated in Figure 7.7a, the shape of the moment diagram obtained by the Winkler analysis is much different from that which would be obtained by the standard RIGID analysis. Since the Winkler analysis cannot give reliable information with respect to wall displacements, and since wall displacements and displacements of the ground retained by the wall were critical to performance, it was decided to perform a NLFEM analysis. The NLFEM analysis investigated wall displacements and bending moment demands for each stage of construction.

\subsection{NLFEM Analysis}

\subsubsection{NLFEM analysis objectives}

The purpose of the NLFEM analysis was threefold: (1) to provide a means for additional confirmation of the procedure used in designing the wall, (2) to predict potential wall performance during excavation and tieback installation, and (3) to assist in the interpretation of instrumentation results. The results from the study depict wall behavior in terms of lateral deflection, bending moments, and earth pressures for each stage of construction.

\subsubsection{NLFEM analysis description}

The computer program SOILSTRUCT was used for the analysis.

SOILSTRUCT was specifically developed to model complex soil-structure interaction problems. The finite element mesh developed to model Panel 6 was similar to that shown in Figure 1.6. Descriptions of the elements used in the analysis can be found in Mosher and Knowles (1990).

SOILSTRUCT is designed so that the actual construction process can be simulated. Simulation of the actual sequence of construction is important because the soil stress response is nonlinear and stress path dependent. SOILSTRUCT provides for simulation of initial stresses, fill placement, material excavation, dewatering, and placement of structural materials in a series of incremental load steps. Incremental stresses and displacements are computed after each load step. Table 7.2 lists the loading steps used to model the sequence for the wall construction and lock channel excavation. 


\subsubsection{NLFEM analysis results}

7.7.3.1 General. The results for each stage of construction were studied, from the in situ state and wall construction through the excavation and tie installation procedure. The results for each stage of construction are illustrated in Figures 7.8a-7.8i and described below in terms of earth pressures, wall bending moments, and displacements.

\begin{tabular}{|c|c|}
\hline \multicolumn{2}{|c|}{$\begin{array}{l}\text { Table } 7.2 \\
\text { Loading Steps in SOILSTRUCT Analysis }\end{array}$} \\
\hline $\begin{array}{l}\text { Step } \\
\text { No. }\end{array}$ & Description \\
\hline 1 & Construct surcharge to pre-excavation grade (four increments) \\
\hline 2 & Excavate for railroad relocation \\
\hline 3 & Construct slurry trench temporary tieback wall \\
\hline 4 & Excavate in front of wall to elev $78.5 \mathrm{ft}(23.9 \mathrm{~m})$ \\
\hline 5 & $\begin{array}{l}\text { Install upper tieback anchor at elev } 84 \mathrm{ft}(25.6 \mathrm{~m}) \text { and prestress to } 150 \text { percent } \\
\text { of the design load }\end{array}$ \\
\hline 6 & Excavate in front of wall to elev $67.5 \mathrm{ft}(20.6 \mathrm{~m})$ and lock off upper anchor at design load \\
\hline 7 & $\begin{array}{l}\text { Install second tieback anchor at elev } 73 \mathrm{ft}(22.3 \mathrm{~m}) \text { and prestress to } 150 \text { percent } \\
\text { of the design load }\end{array}$ \\
\hline 8 & $\begin{array}{l}\text { Excavate in front of wall to elev } 56.5 \mathrm{ft}(17.2 \mathrm{~m}) \text { and lock off second anchor at design } \\
\text { load }\end{array}$ \\
\hline 9 & $\begin{array}{l}\text { Install third tieback anchor at elev } 62 \mathrm{ft} 18.9 \mathrm{~m} \text { ) and prestress to } 150 \text { percent of the } \\
\text { design load }\end{array}$ \\
\hline 10 & Excavate in front of wall to elev $45 \mathrm{ft}(13.7 \mathrm{~m})$ and lock off third anchor at design load \\
\hline 11 & $\begin{array}{l}\text { Install fourth tieback anchor at elev } 51 \mathrm{ft}(15.5 \mathrm{~m}) \text { and prestress to } 150 \text { percent of design } \\
\text { load }\end{array}$ \\
\hline 12 & Excavate to bottom of wall at elev $39 \mathrm{ft}(11.9 \mathrm{~m})$ and lock off fourth anchor at design load \\
\hline
\end{tabular}

7.7.3.2 Earth pressures. Lateral earth pressures on the wall for each stage of construction are illustrated in Figures 7.8a-7.8i. The initial pressure on the wall is approximately 50 percent greater than at-rest pressure. This increase can be attributed to overconsolidation and replacement of the soil by a concrete wall. The dotted line represents at-rest pressure increased by 50 percent. The earth pressure distribution changes throughout construction as a result of excavation and anchor prestressing. After the first excavation to elevation $78.5 \mathrm{ft}(23.9 \mathrm{~m})$, the soil behind and near the top of the wall is in an active state as a result of the wall moving toward the excavation. Farther down the wall, the lateral earth pressure is greater than active, but less than the initial pressure on the wall. The analysis shows that, with prestressing of the first anchor, earth pressures increase to greater than initial pressures behind the upper one-third of the wall. Subsequent excavations and anchor prestressings show decreases and increases, respectively, of the earth pressures along the wall. Bulging of pressures around the anchors appears in the lower one-half of the wall. Although the shape of the pressure diagram is 
approximately trapezoidal during the initial stages of construction, it is closer to triangular for the later stages of construction.

7.7.3.3 Wall bending moments. Wall bending moments for each stage of construction are illustrated in Figures 7.8a-7.8i. Except for the first excavation, the moment diagram for the wall retains the same general form throughout construction. The region immediately behind the upper anchor experiences negative bending moment, and the lower region below the upper anchor experiences positive bending moment. The maximum moment is always positive and varies during construction with a maximum value of about $180 \mathrm{kN} \cdot \mathrm{m}$ (133 ft-kips). The maximum moment develops during the intermediate stages of excavation after the second anchor is prestressed.

7.7.3.4 Wall displacements. Wall displacements for each stage of construction are illustrated in Figures 7.8a-7.8i. With the first excavation to elevation $78.5 \mathrm{ft}(23.9 \mathrm{~m})$, the wall moves $0.5 \mathrm{in}$. $(13 \mathrm{~mm})$ toward the excavation. With prestressing of the upper tieback anchor, the wall is pulled back into the retained soil, resulting in a displacement of $0.78 \mathrm{in} .(20 \mathrm{~mm})$ past vertical in a direction away from the excavation. In subsequent construction steps there is little change in the deflected position of the wall. In general, the wall moves into the soil when each anchor is prestressed and back toward vertical with each excavation.

\subsubsection{Conclusions}

It has been demonstrated through the NLFEM analysis of the Bonneville Navigation Lock temporary tieback wall that the behavior assumed with respect to the RIGID analysis of flexible tieback wall systems is inconsistent with the behavior observed for the temporary tieback wall. The reasons are numerous; a few are cited below.

- The wall is stiff rather than flexible.

- Prestressing to achieve stringent displacement control objectives results in total pressures in excess of at-rest conditions.

- Construction sequencing has a major impact on wall performance.

- Pressure distributions are approximately triangular, especially during the final stages of construction. 


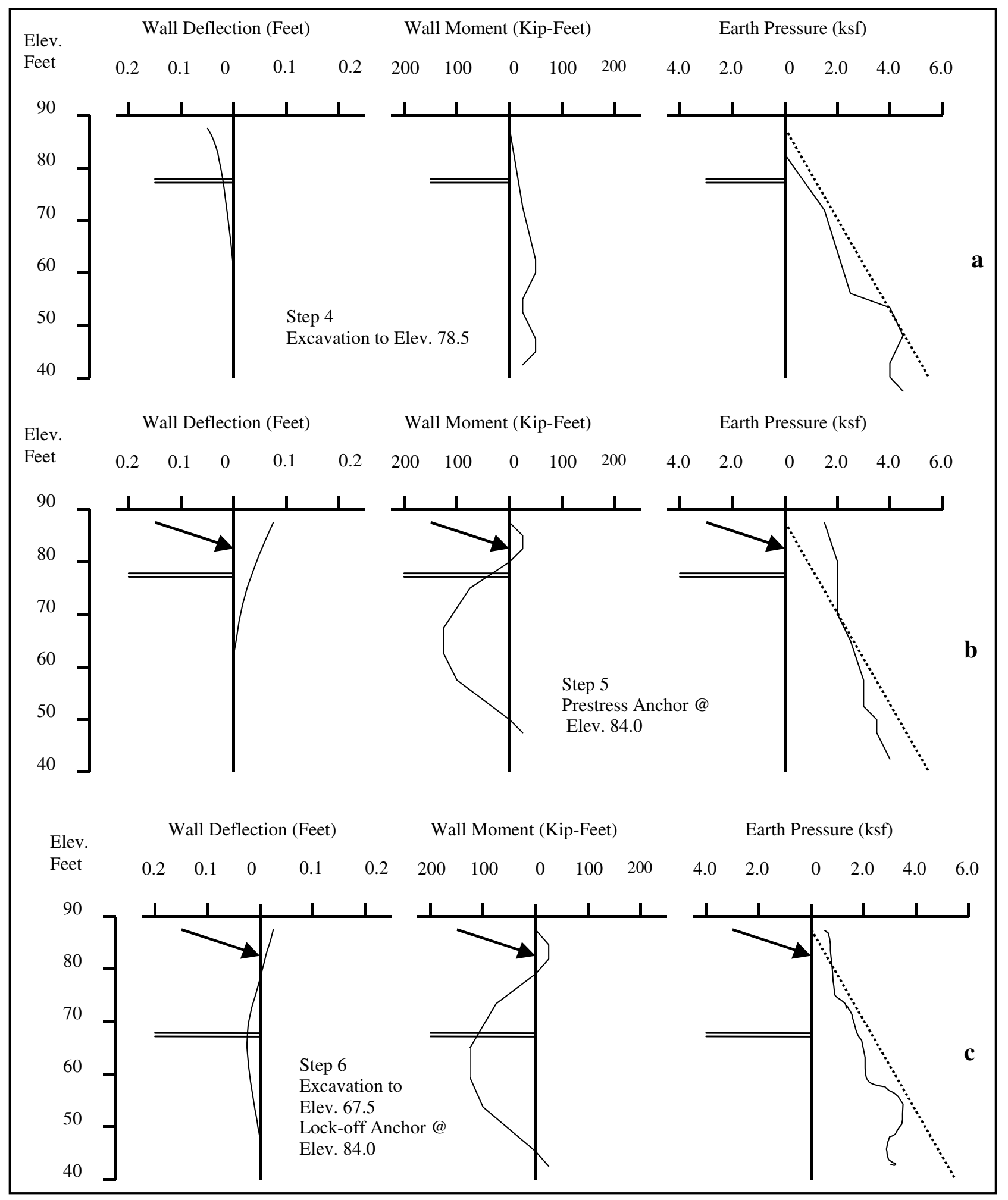

Figure 7.8. Deflections, moments, and earth pressures for Bonneville Navigation Lock, temporary tieback wall - Panel 6 (Sheet 1 of 3) 


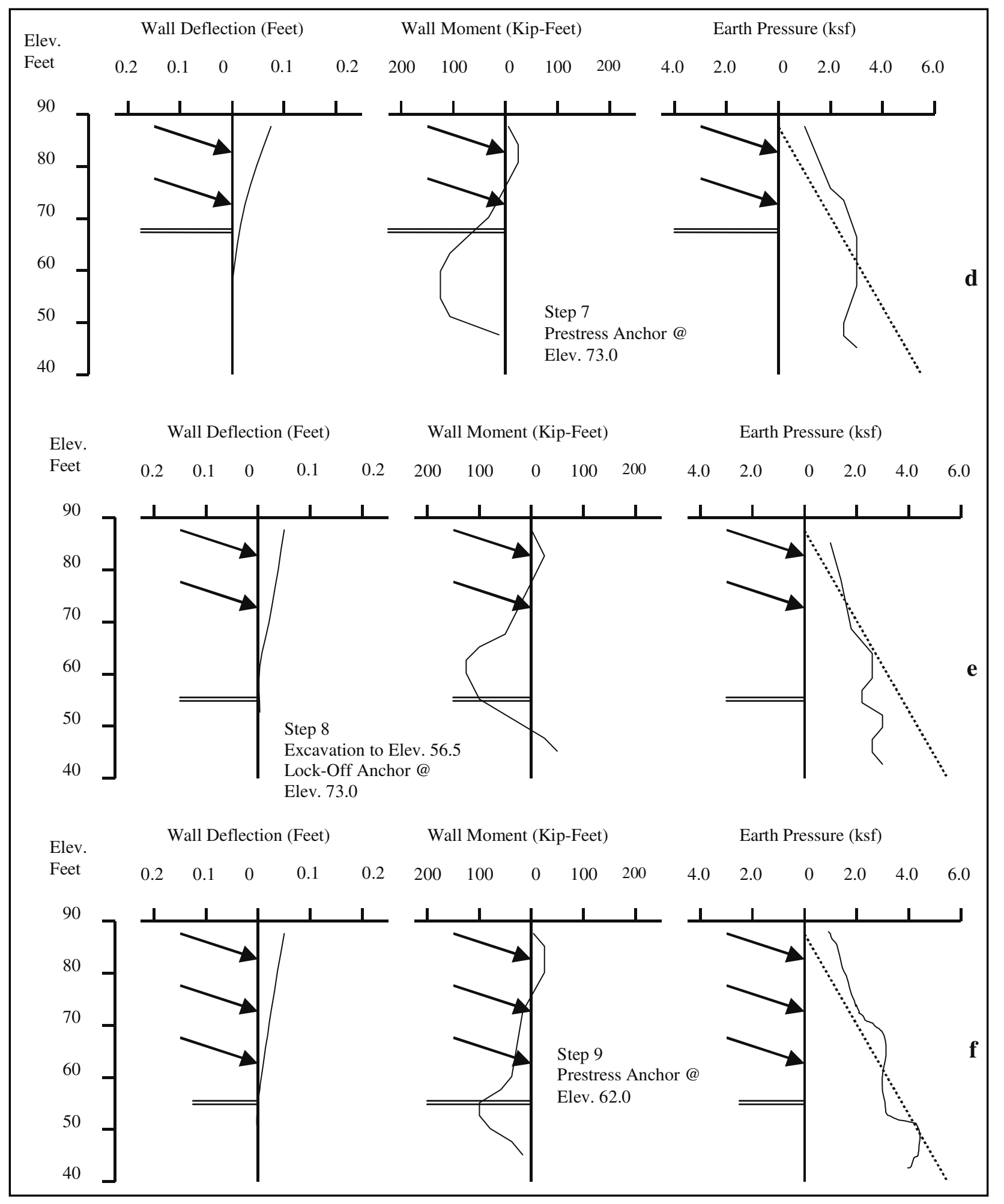

Figure 7.8. (Sheet 2 of 3) 


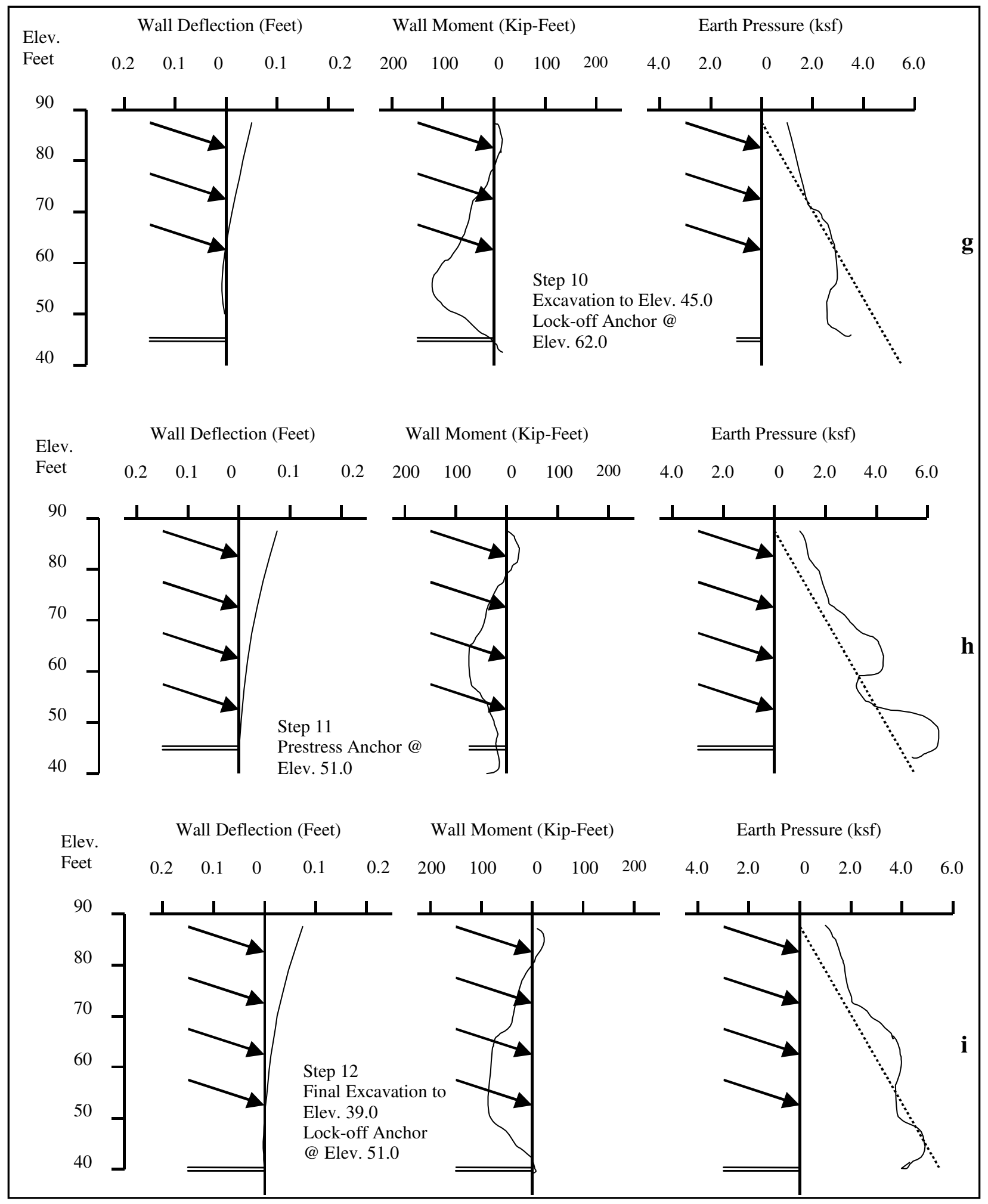

Figure 7.8. (Sheet 3 of 3 ) 


\section{Design of Tieback Wall System Components and Wall Toe}

\subsection{Ground Anchors}

The following information on ground anchors basically follows the recommendations of Sabatini, Pass, and Bachus (1999).

\subsubsection{General}

A prestressed grouted ground anchor is a structural element installed in soil or rock that is used to transmit an applied tensile load to the ground. Grouted ground anchors, referred to simply as ground anchors (or "tiebacks") are installed in grout-filled holes. The basic components of a ground anchor include the anchorage, the free stressing (unbonded) length, and the bond length. These and other components of a ground anchor are shown schematically in Figure 8.1.

The anchorage is the combined system of anchor head, bearing plate, and trumpet that is capable of transmitting the prestress force from the prestressing steel (bar or strand) to the tieback wall. The unbonded length is that portion of the prestressing steel that is free to elongate elastically and transfer the resisting force from the bond length to the tieback wall. A bondbreaker is a smooth plastic sleeve that is placed over the tendon in the unbonded length to prevent the prestressing steel from bonding to the surrounding grout. The bondbreaker enables the prestressing steel in the unbonded length to elongate without obstruction during testing and stressing, and leaves the prestressing steel unbonded after lock-off. The tendon bond length is that length of the prestressing steel that is bonded to the grout and is capable of transmitting the applied tensile force to the ground. The anchor bond length should be located behind the critical failure surface.

A portion of the complete ground anchor assembly is referred to as the tendon. The tendon includes the prestressing steel element (strands or bars), corrosion protection, sheaths (also referred to as sheathings), centralizers, and spacers, but specifically excludes the grout. The definition of a tendon, as described in PTI (1996), also includes the anchorage; however, it is assumed herein that the tendon 


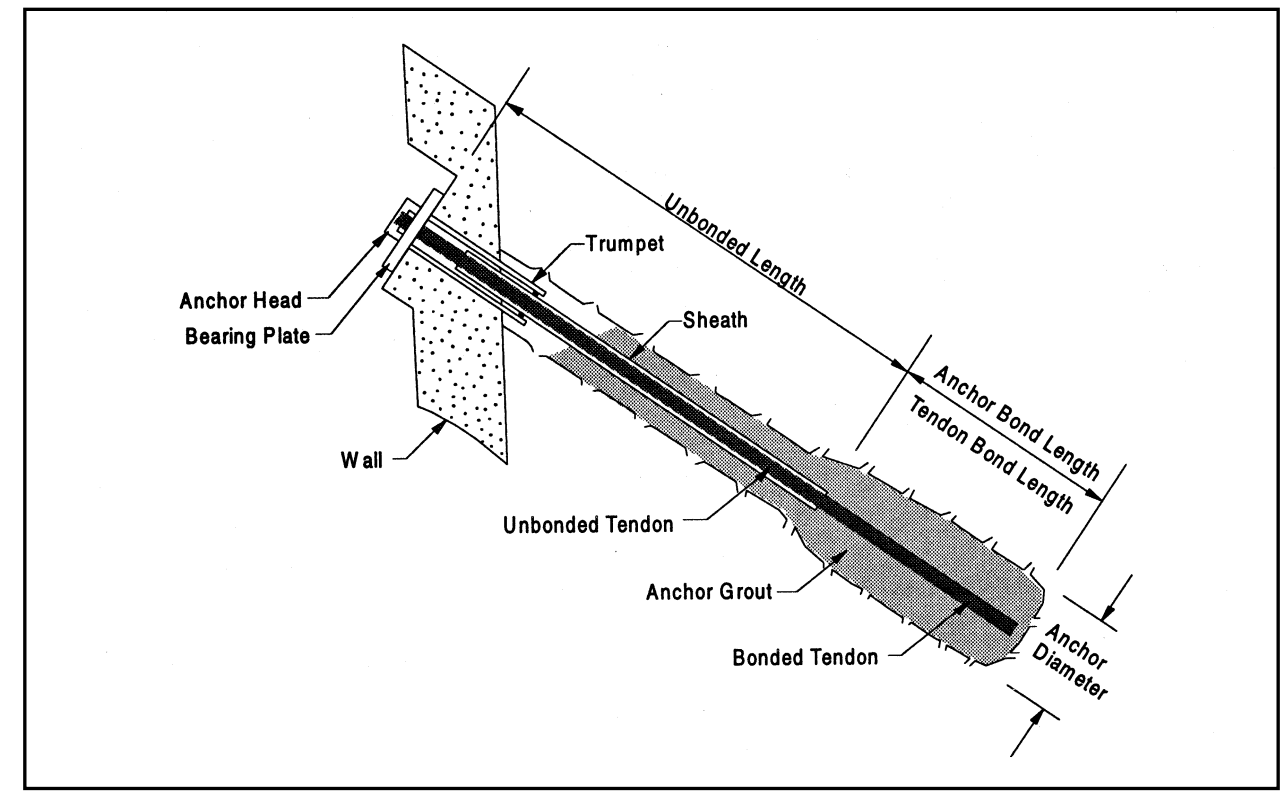

Figure 8.1. Components of a ground anchor (after Figure 1 of Sabatini, Pass, and Bachus 1999)

does not include the anchorage. The sheath is a smooth or corrugated pipe or tube that protects the prestressing steel in the unbonded length from corrosion. Centralizers position the tendon in the drill hole such that the specified minimum grout cover is achieved around the tendon. For multiple-element tendons, spacers are used to separate the strands or bars of the tendons so that each element is adequately bonded to the anchor grout. The grout is a portland cement-based mixture that provides load transfer from the tendon to the grout and provides corrosion protection for the tendon.

\subsubsection{Types of ground anchors}

Three main ground anchor types are currently used in the United States: (1) straight-shaft gravity-grouted ground anchors (Type A); straight-shaft pressure-grouted ground anchors (Type B); and (3) post-grouted ground anchors (Type C). Although not common today in U.S. practice, another type of anchor is the under-reamed anchor (Type D). These ground anchor types are illustrated in Figure 8.2 and are briefly described in the following sections.

8.1.2.1 Straight-shaft gravity-grouted ground anchors. Straight-shaft gravity-grouted anchors are typically installed in rock and very stiff to hard cohesive soil deposits using either rotary drilling or hollow-stem auger methods. Tremie (gravity displacement) methods are used to grout the anchor in a straightshaft borehole. The borehole may be cased or uncased depending on the stability of the borehole. Anchor resistance to pullout of the grouted anchor depends on the shear resistance that is mobilized at the grout/ground interface. 


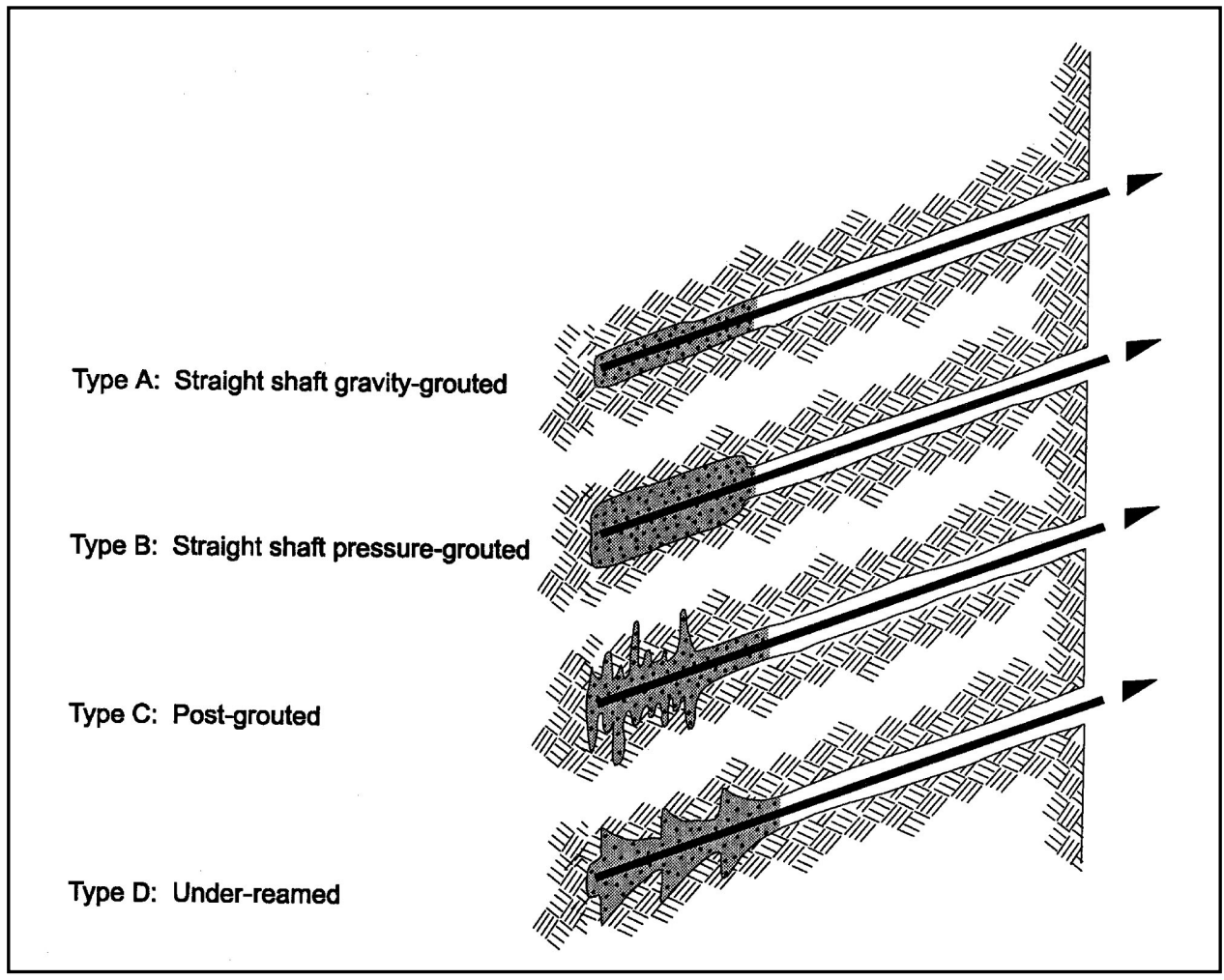

Figure 8.2. Main types of grouted ground anchors (after Figure 4 of Sabatini, Pass, and Bachus 1999)

8.1.2.2 Straight-shaft pressure-grouted ground anchors. Straight-shaft pressure-grouted anchors are most suitable for coarse granular soils and weak fissured rock. This anchor type is also used in fine-grained cohesionless soils. With this type of anchor, grout is injected into the bond zone under pressures greater than $50 \mathrm{psi}(345 \mathrm{kPa})$. The borehole is typically drilled using a hollowstem auger or rotary techniques with drill casings. As the auger or casing is withdrawn, the grout is injected into the hole under pressure until the entire anchor bond length is grouted. This grouting procedure increases resistance to pullout relative to tremie grouting methods by (1) increasing the normal stress (i.e., confining pressure) on the grout bulb resulting from compaction of the surrounding material locally around the grout bulb and (2) increasing the effective diameter of the grout bulb.

8.1.2.3 Post-grouted ground anchors. Post-grouted ground anchors use delayed multiple grout injections to enlarge the grout body of the gravity-grouted ground anchors. Each injection is separated by 1 or 2 days. Post-grouting is accomplished through a sealed grout tube installed with the tendons. The tube is equipped with check valves in the bond zone. The check valves allow additional grout to be injected under high pressure into the initial grout, which has set. The high-pressure grout fractures the initial grout and wedges it outward into the soil, enlarging the grout body. Two fundamental types of post-grout anchors are used. One system uses a packer to isolate each valve. The other system pumps the grout down the post-grout tube without controlling which valves are open. 
8.1.2.4 Under-reamed anchors. Under-reamed anchors consist of tremiegrouted boreholes that include a series of enlargement bells or under-reams. This type of anchor may be used in firm to hard cohesive deposits. In addition to resistance through side shear, as is the principal load transfer mechanism for other anchors, resistance may also be mobilized through end bearing. Care must be taken to form and clean the under-reams.

\subsubsection{Tendon materials}

Both bar and strand tendons are commonly used for soil and rock anchors. Material specifications for bar and strand anchors are codified in American Society for Testing and Materials (ASTM) A722 and ASTM A416, respectively. Indent strand is codified in ASTM A886. Bar tendons are commonly available in sizes ranging from 1 to $2.5 \mathrm{in}$. (26 to $64 \mathrm{~mm}$ ) in diameter. Uncouple bar lengths up to $60 \mathrm{ft}(18 \mathrm{~m})$ are available. When longer lengths are needed, bar sections can be coupled. Anchor design loads up to 467 kips $(2,077 \mathrm{kN})$ can be resisted by a single 2.5-in. (64-mm)-diam tendon. As compared to strand tendons, bars are easier to stress, and their loads can be adjusted after lock-off. Strand tendons consist of multiple seven-wire strands. The common strand in U.S. practice is $0.5 \mathrm{in} .(13 \mathrm{~mm})$ in diameter. Anchors using multiple strands have no practical load or anchor length limitations.

\subsection{Ground Anchor Design}

\subsubsection{Introduction}

In accordance with Sabatini, Pass, and Bachus (1999), this section presents procedures that are commonly used to locate the critical potential failure surface and to calculate the ground anchor loads from apparent pressure diagrams, the design of the unbonded and bonded lengths of the anchor, the allowable load requirements for the prestressing steel element, and the horizontal and vertical spacing and inclination of the anchor.

\subsubsection{Location of critical potential failure surface}

The location of the critical potential failure surface must be evaluated since the anchor bond zone must be located sufficiently behind the critical potential failure surface so that the load is not transferred from the anchor bond zone into the "no-load" zone. The no-load zone is defined as the zone between the critical potential failure surface and the wall, and is also referred to as the unbonded length. The unbonded length is typically extended either a minimum distance of

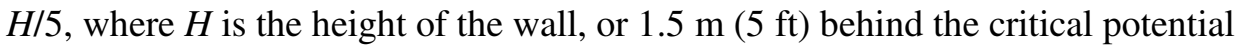
failure surface. Minimum requirements on the unbonded length of the anchor and the location of the anchor bond zone are described below.

For walls constructed in cohesionless soils, the critical potential failure surface can be assumed to extend up from the corner of the excavation at an angle of 
$45 \mathrm{deg}+\phi^{\prime} / 2$ from the horizontal (i.e., the active wedge). The sliding wedge force equilibrium method presented in Chapter 9 may also be used to more accurately evaluate the location of the critical potential surface. Limit equilibrium methods and trial wedge analysis may be used for general ground conditions and can incorporate surcharge loadings and variable soil stratigraphies.

\subsubsection{Calculation of ground anchor loads from apparent pressure diagrams}

Ground anchor loads for flexible anchored wall applications can be estimated from apparent earth pressure envelopes. Methods commonly used-the tributary area method and the hinge method-were developed to enable hand calculations to be made for statically indeterminate systems. Both methods, when used with appropriate earth pressure diagrams, have provided reasonable estimates of ground anchor loads and wall bending moments for anchored wall systems constructed in competent soils. The calculations for horizontal ground anchor loads using the tributary area method are illustrated in Chapter 5. For flexible wall systems, the total load approach described in Chapter 5 is often used to construct the apparent pressure diagram and, based on the apparent pressure diagram, the horizontal ground anchor loads are determined.

The procedures used to determine the anchor loads for stiffer wall systems are not specifically discussed in Sabatini, Pass, and Bachus (1999). For the stiffer wall systems the total earth load is often based on conditions approaching "atrest." Often, a triangular distribution of the total earth load is used to determine anchor loads since the apparent pressure diagram will usually underpredict the lowermost anchor loads on the stiffer wall systems. In addition, a trapezoidal distribution of the total earth load (apparent pressure diagram) may be used to estimate the loads for the uppermost anchors.

Information on the calculation of horizontal ground anchor loads using the hinge method can be found in Sabatini, Pass, and Bachus (1999). Both the tributary area method and the hinge method assume that a hinge (i.e., zero bending moment) develops at the excavation subgrade and that the excavation subgrade acts as a strut support. This latter assumption is reasonable for walls that penetrate into competent materials. The maximum bending moment that controls the design of the wall typically occurs in the exposed portion of the wall, i.e., above the excavation subgrade. For walls constructed in competent materials, a reaction force, $R$, is assumed to be supported by the passive resistance of the soil below the excavation subgrade. In general, the wall must be embedded sufficiently deep to provide a passive resistance capacity equal to or greater than 1.5 times the reaction force, $R$. This embedment depth will vary depending on whether the wall is continuous below grade or consists of discrete elements (soldier beams). It will also depend on the strength of the soil or rock materials in which the wall is embedded. Embedments deeper than that necessary to satisfy factor of safety requirements may be desirable in order to minimize wall displacements and rotations. 
For walls that penetrate weak materials, sufficient passive capacity below the base of the excavation may not be available to resist the reaction force regardless

of the wall embedment depth. For that case, the lowest anchor may be designed to carry the same load as defined for the lowest anchor plus the load corresponding to the reaction force. Alternatively, soil-structure interaction analyses (e.g., beam on elastic foundation, linear FEM, nonlinear FEM) may be used to design continuous beams with small toe reactions, as it may be overly conservative to assume that all load is carried by the lowest anchor.

The values determined for the anchor loads, as a result of earth pressures, water pressures, and surcharge loadings, is generally the horizontal component of the anchor load per unit width of wall, $T_{h i}$. The total horizontal anchor load, $T_{h}$, is then calculated as

$$
T_{h}=T_{h i}(s)
$$

where $s$ is the horizontal spacing between adjacent anchors.

The anchor load, $T$, to be used in sizing the anchor and its components and in designing the anchor bond zone (i.e., the design load) is calculated as

$$
T=\frac{T_{h}}{\cos \theta}
$$

where $\theta$ is the angle of inclination of the anchor below horizontal.

The vertical component of the anchor load is calculated as

$$
T_{v}=T \sin \theta
$$

\subsubsection{Design of the unbonded length}

The minimum unbonded length for rock and soil ground anchors is $4.5 \mathrm{~m}$

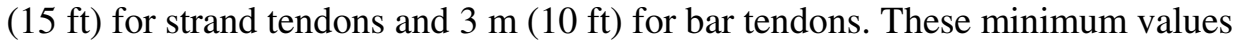
are intended to prevent significant reductions in load resulting from seating losses during transfer of load to the structure following anchor load testing. Longer unbonded lengths may be required to (1) locate the bond length a minimum distance behind the critical potential failure surface; (2) locate the anchor bond zone in ground suitable for anchoring; (3) ensure overall stability of the anchored system; and (4) accommodate long-term movements. In general, the unbonded length is extended a minimum distance of $H / 5$ or $1.5 \mathrm{~m}(5 \mathrm{ft})$ behind the critical potential failure surface to accommodate minor load transfer to the grout column above the top of the anchor bond zone.

As a general rule, the anchor bond zone and unbonded zone should be grouted in one stage to maintain hole stability and to create a continuous grout cover for corrosion protection. However, for large-diameter anchors in which the 
unbonded length of the anchor extends just behind the critical potential failure surface, significant strains at the top of the anchor bond zone may cause load transfer into the grout column above the anchor bond zone. Large-diameter anchors have been grouted in two stages (two-stage grouting). With two-stage grouting, the anchor bond length is grouted (Stage 1) and the anchor is tested. The unbonded length portion of the drill hole is then grouted (Stage 2) after the anchor is tested. The two-stage procedure is not recommended since local collapse of the ground can occur, which will compromise the corrosion protection provided by the grout.

\subsubsection{Compression anchors}

Compression anchors are anchors in which the grout body in the bond length is, at least partially, loaded in compression. For a typical tension ground anchor (Figure 8.1), the anchor bond length and tendon bond length are the same. For typical tension ground anchors, load is transferred through bond first at the top of the anchor bond length and, with continued loading (prestressing), bond-slip causes load transfer to progress downward to the bottom of anchor bond length. For single-stage grouted tension anchors, because load is first transferred to the top of the anchor bond zone, there is the potential for load transfer into the noload zone (i.e., that area of the tendon between the structure and the assumed failure plane). This is especially a concern for large-diameter anchors installed in some cohesive soils for which relatively large residual movements are required to develop bond at the grout/ground interface.

Two types of compression anchors have been used. These include a ground anchor fitted with an end plate (Figure 8.3a) and a composite design where the top of the tendon bond length is extended a certain distance into the anchor bond length (Figure 8.3b).

During stressing, the entire grout column for the endplate compression anchor is loaded in compression. For the composite design, the portion of the anchor grout located above the tendon bond length is loaded in compression. The use of compression-type anchors minimizes the load transferred above the anchor bond zone into the no-load zone. Compression anchor design should consider expected levels of compressive strain in the grout body. Strains should be within tolerable limits to minimize the potential for the grout to fail due to compression loading. Where compression anchors are to be used for a permanent application, a predesign test program may be warranted unless the behavior and satisfactory performance of the proposed compression anchor has been verified through prior experience or research results.

Compression anchors are not commonly used for small-diameter anchors in cohesionless deposits, but may be used for large-diameter anchors in cohesive soils. In cohesive soils, composite design of compression anchors typically involves a tendon bond length equal to between 50 and 100 percent of the anchor bond length. 


\subsubsection{Design of the anchor bond length}

Estimates of load transfer capacity in the anchor bond length are typically based on previous field experience. When estimating capacities using previous field results, potential variations in capacity due to differing installation and grouting methods must be considered. In a given soil deposit, the actual capacity in the field will depend on the method of drilling, including quality of drill hole cleaning and period of time that the drill hole is left open, the diameter of the drill hole, the method and pressure used in grouting, and the length of the anchor bond zone. Except for certain minimum values, the selection of these items should be left to the discretion of the specialty anchor contractor.

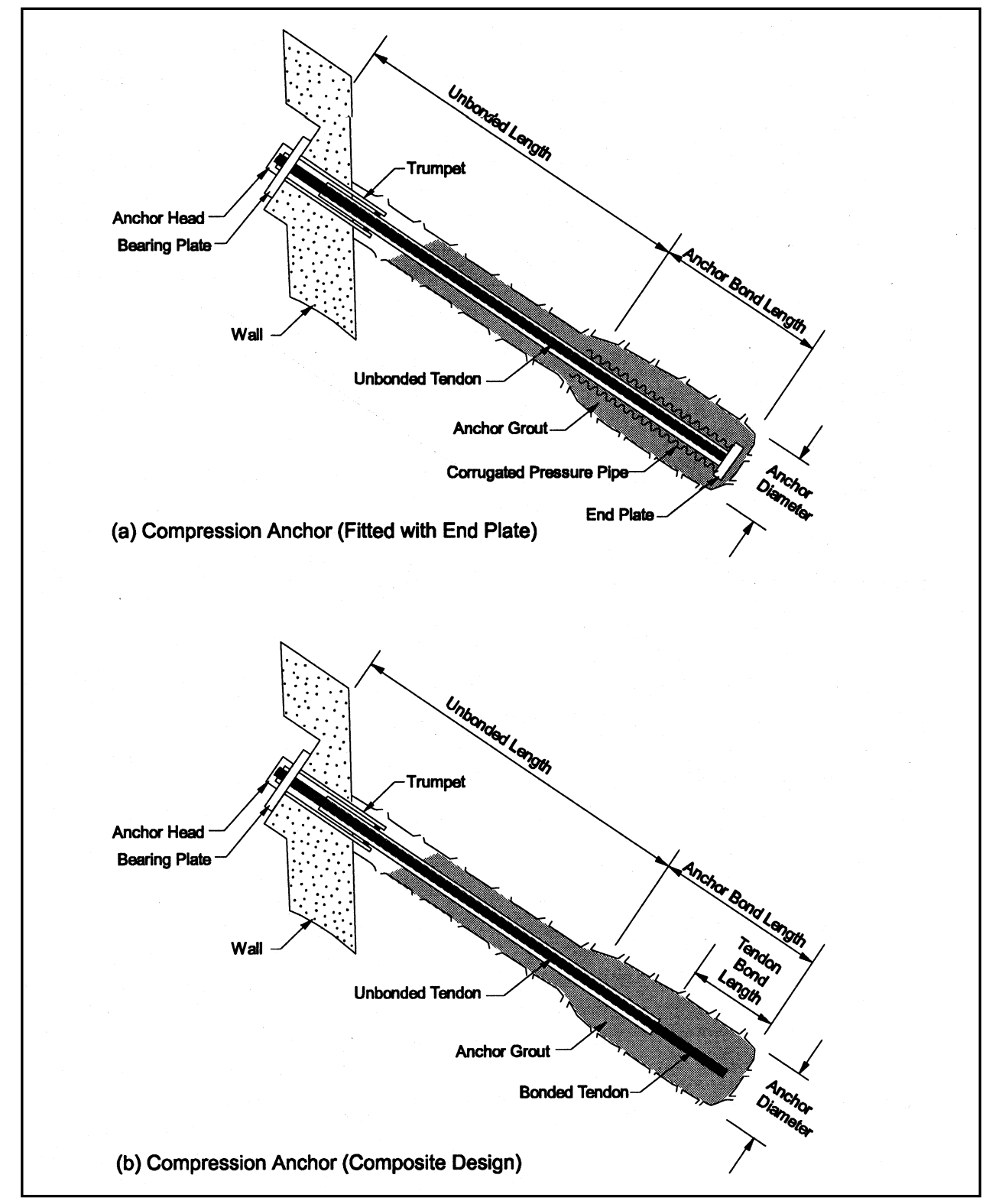

Figure 8.3. Types of compression anchors (after Figure 35 of Sabatini, Pass, and Bachus 1999) 
The main responsibility for the designer is to define a minimum anchor capacity that can be achieved in a given ground type. Therefore, estimation of anchor capacity should be based on the simplest commonly installed anchor, i.e., the straight-shaft gravity-grouted anchor. Estimates made assuming that this anchor will be installed will produce a design capacity that may confidently be achieved while allowing the specialty contractors to use more effective and/or economical anchoring methods to achieve the specific capacity. The design capacity of each anchor will be verified by testing before acceptance.

Many tieback wall projects consisting of vertical sheet-pile/wale/posttensioned tieback anchor-type systems or soldier beam/lagging/P-T tieback anchor-type systems have been completed using small-diameter, straight-shaft gravity-grouted anchors. Because of the similarity of many such projects, some fairly typical anchor characteristics can be summarized. These are intended to provide a range of typical design values for the above-mentioned systems to engineers who are unfamiliar with anchor design. Design loads and anchor lengths may be greater than indicated below for high-capacity slurry trench wall systems and high-capacity secant pile systems.

8.2.6.1 Anchor design loads. In general, anchor design loads for vertical sheet-pile/wale/P-T tieback anchor-type systems and soldier beam/lagging/P-T tieback anchor-type systems range between 260 and 1,160 kN (60 and 260 kips). Anchor tendons of this capacity can be handled without the need for unusually heavy or specialized equipment. In addition, stressing equipment can be handled by one or two workers without the aid of mechanical lifting equipment. The drill hole diameter is generally less than $150 \mathrm{~mm}$ (6 in.), except for hollow-stem augured anchors, which are typically $300 \mathrm{~mm}$ (12 in.) in diameter.

8.2.6.2 Anchor lengths. Generally the total anchor length varies between 9 and $18 \mathrm{~m}$ (30 to $60 \mathrm{ft}$ ), although anchors in lengths up to and exceeding $60 \mathrm{~m}$ (200 ft) have been used. Because of geotechnical or geometrical requirements, few anchors for walls are less than $9 \mathrm{~m}$ (30 ft) long. A minimum unbonded length

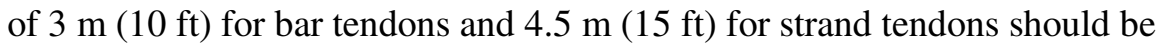
adopted. These minimum unbonded lengths are required to avoid unacceptable load reduction resulting from seating losses during load transfer and prestress losses due to creep in the prestressing steel or the soil.

8.2.6.3 Ground anchor inclination. Ground anchors are generally installed at angles of 15 to 30 deg below the horizontal, although angles of 10 to $45 \mathrm{deg}$ are within the capabilities of most contractors. Regardless of the anchor inclination, the anchor bond zone must be developed behind potential slip surfaces and in soil or rock layers that can develop the necessary design load. Steep inclinations may be necessary to avoid underground utilities, adjacent foundations, right-of-way constraints, or weak soil or rock layers. Anchors should be installed as close to horizontal as possible to minimize vertical loads resulting from anchor lock-off loads; however, grouting of anchors installed at angles less than $10 \mathrm{deg}$ is not common unless special grouting techniques are used. 


\subsubsection{Anchor selection}

For a specific project, the first step in estimating the minimum allowable capacity is to assume a maximum anchor bond length. In the case of a site with no restrictions on right-of-way, a 15-deg inclination of the anchor should be assumed

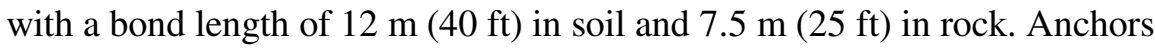
founded in soil and rock should be designed assuming the entire embedment in soil (i.e., assuming a bond length equal to $12 \mathrm{~m}$ (40 ft).

8.2.7.1 Soil anchors. For the purposes of preliminary design (Sabatini, Pass, and Bachus 1999), the ultimate load transferred from the bond length to the soil may be estimated for a small-diameter, straight gravity-grouted anchor from the soil type and density (or Standard Penetration Test (SPT) resistances blowcount value) in accordance with Table 8.1. The maximum allowable design load in soil may be determined by multiplying the bond length by the ultimate transfer load and dividing by a factor of safety. The factor of safety for soil anchors is usually equal to 2 .

\begin{tabular}{|c|c|c|c|}
\hline \multicolumn{4}{|c|}{$\begin{array}{l}\text { Table } 8.1 \\
\text { Presumptive Ultimate Values of Load Transfer for Preliminary } \\
\text { Design of Small-Diameter Straight-Shaft Gravity-Grouted Ground } \\
\text { Anchors in Soil (after Table } 6 \text { of Sabatini, Pass, and Bachus 1999) }\end{array}$} \\
\hline \multirow[b]{2}{*}{ Soil Type } & \multirow{2}{*}{$\begin{array}{l}\text { Relative Density/Consistency } \\
\text { (SPT Range) }^{1}\end{array}$} & \multicolumn{2}{|c|}{$\begin{array}{c}\text { Estimated Ultimate } \\
\text { Transfer Load }\end{array}$} \\
\hline & & $\mathrm{kN} / \mathrm{m}$ & kips/ft \\
\hline 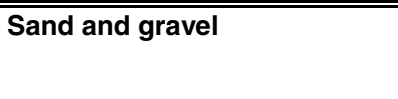 & $\begin{array}{l}\text { Loose (4-10) } \\
\text { Medium dense (11-30) } \\
\text { Dense (31-50) }\end{array}$ & $\begin{array}{l}145 \\
220 \\
290\end{array}$ & $\begin{array}{l}10 \\
15 \\
20\end{array}$ \\
\hline Sand & $\begin{array}{l}\text { Loose }(4-10) \\
\text { Medium dense (11-30) } \\
\text { Dense }(31-50)\end{array}$ & $\begin{array}{l}100 \\
145 \\
190\end{array}$ & $\begin{array}{c}20 \\
7 \\
10 \\
13\end{array}$ \\
\hline Sand and silt & $\begin{array}{l}\text { Loose (4-10) } \\
\text { Medium dense (11-30) } \\
\text { Dense (31-50) }\end{array}$ & $\begin{array}{r}70 \\
100 \\
130\end{array}$ & $\begin{array}{l}5 \\
7 \\
9\end{array}$ \\
\hline $\begin{array}{l}\text { Silt-clay mixture with low } \\
\text { plasticity or fines micaceous } \\
\text { sand or silt mixtures }\end{array}$ & $\begin{array}{l}\text { Stiff }(10-20) \\
\text { Hard }(21-40)\end{array}$ & $\begin{array}{l}30 \\
60\end{array}$ & $\begin{array}{l}2 \\
4\end{array}$ \\
\hline
\end{tabular}

Anchor bond lengths for gravity-grouted, pressure-grouted, and post-grouted anchors are typically 4.5 to $12 \mathrm{~m}$ ( 15 to $40 \mathrm{ft}$ ) since significant increases in

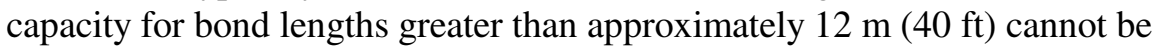
achieved unless specialized methods are used to transfer load from the top of the anchor bond zone toward the end of the anchor. For bond zones that function in tension, initial load increments transferred to the anchor bond zone are resisted by the soil near the top of the anchor bond zone as strains occur in the upper body of the grout (Figure 8.4). As additional increments of load are transferred to the anchor bond zone, the strains in the top of the anchor bond zone may exceed the peak strain for strain-sensitive soils. In that case, the bond stress begins to decrease at the top and the peak strain shifts down the anchor body. In strainsensitive soils, the shape of the stress-strain curve diagram will determine the actual bond length where significant load is mobilized. Attempts to mobilize 


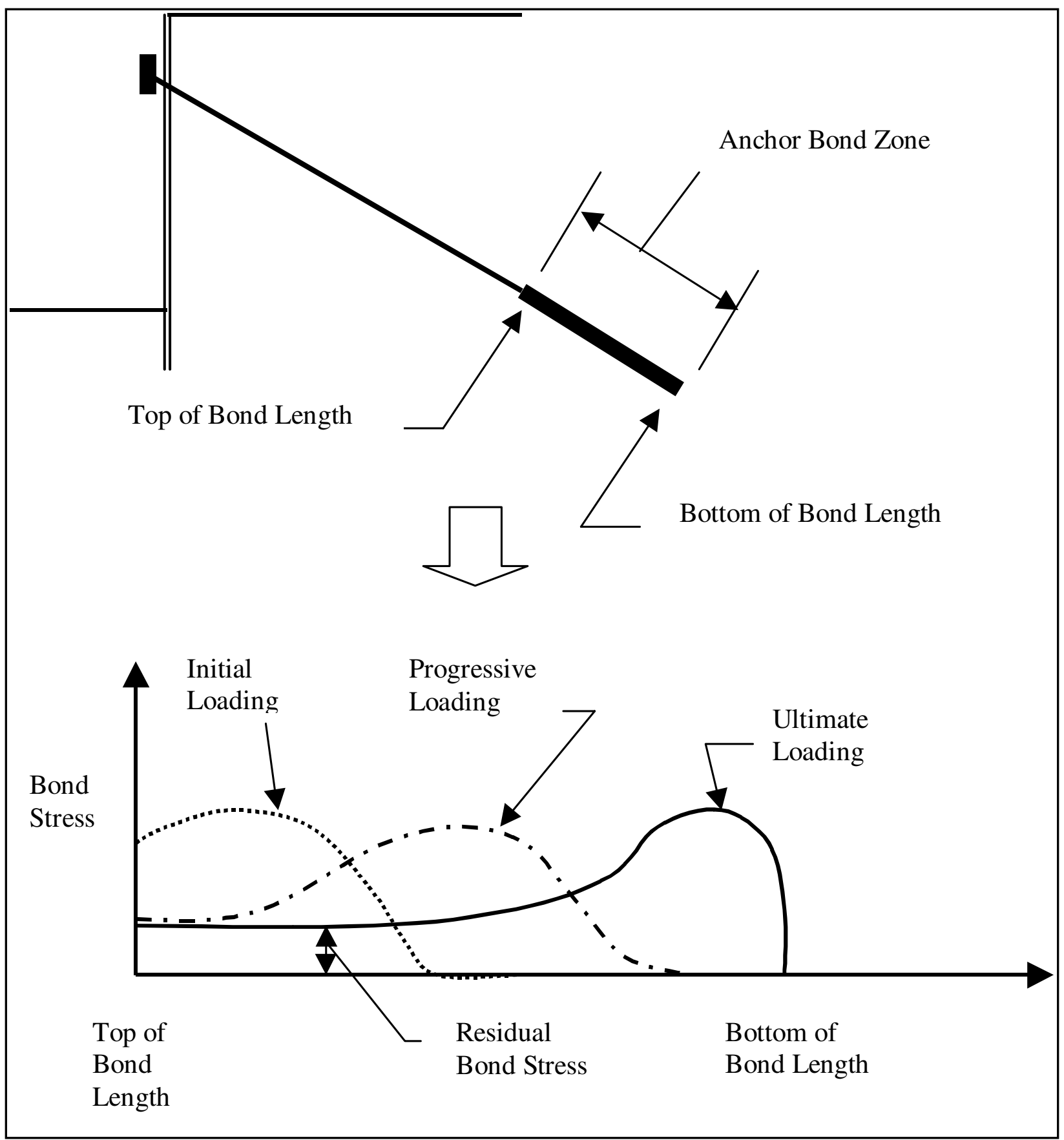

Figure 8.4. Mobilization of bond stress for a tension anchor in anchor bond zone

larger portions of the bond length will result in small increases in capacity as residual load transfer values develop at the top and the peak value shifts toward the bottom.

Pressure grouting in cohesionless soils significantly increases the normal stresses acting on the grout body (i.e., increases confinement). Small increases may also be observed in the effective diameter of the anchor body zone, but 
capacity estimates should be based on the as-drilled hole diameter. A range of ultimate bond stress values that have been measured for gravity- and pressuregrouted soil anchors is provided in Tables 8.2a, 8.2b, and 8.2c for rock, cohesive soil, and cohesionless soil, respectively, to show the variation in field-measured ultimate values. When reviewing ultimate bond stress values such as those presented in the aforementioned tables, it is important to recognize that large bond stress values do not necessarily imply a corresponding large anchor capacity per unit length. For example, a hollow-stem augured anchor can develop more capacity per unit length than a small-diameter, post-grouted anchor due primarily to the anchor diameter, not the bond stress value.

Pressure grouting can be effective in increasing capacity in cohesive soils; however, post-grouting is a more effective means of increasing capacity in cohesive soils. Post-grouting increases the radial stresses acting on the grout body and causes an irregular surface to be developed around the bond length, which tends to interlock the grout and the ground. It is difficult to predict load capacity in post-grouted anchors owing to the complexity of the grouting procedures used. However, post-grouting of ground anchors in cohesive soil can increase load capacity of a straight-shaft anchor by 20 to 50 percent or more per phase of postgrouting, with three phases being the common limit (PTI 1996).

8.2.7.2 Rock anchors. For rock anchors, typical bond lengths range from 3 to $10 \mathrm{~m}$ (10 to $30 \mathrm{ft}$ ) with a minimum of $3 \mathrm{~m}$. The ultimate load transferred from the bond length to competent sound rock may be estimated from the rock type (Table 8.3). Lower values may be recommended after input from a geologist, especially if the rock mass strength is controlled by discontinuities. The maximum allowable design anchor load in competent rock is often determined by multiplying the bond length by the ultimate transfer load and dividing by a factor of safety of 3 . This relatively high value of the factor of safety (compared to that of soil) is used to account for uncertainties associated with potential discontinuities in the rock mass, such as joints, fissures, and clay-filled fissures. In weak rocks such as clay shales, bond stress transfer is relatively uniform as compared to bond stress transfer in more competent rock. These weak rocks may be termed "intermediate geomaterials" and have unconfined compressive strengths defined as varying from 0.5 to $5.0 \mathrm{MPa}$ ( 75 to $750 \mathrm{psi}$ ). Design values for evaluation anchor bond lengths in these materials should use a factor of safety of 2 on the ultimate load transfer value.

Typical ranges of ultimate bond stress values for the rock/grout interface (which have been measured) are provided in Table 8.2a. Alternatively, PTI (1966) suggests that the ultimate bond stress between rock and grout be approximated as 10 percent of the unconfined compressive strength of the rock up to a maximum value for ultimate bond stress of 3.1 $\mathrm{MPa}$ (500 psi).

In the calculation of bond length, the implicit assumption is that the bond at the rock-grout interface is mobilized uniformly. This is unlikely to be the case unless the anchor bond zone is formed in soft or weak rock. For conditions where the ratio of the elastic modulus of the grout to the elastic modulus of the rock is less than 1 (e.g., in competent rock), load is transferred from the tendon to the rock in only the upper 1.5 to $3 \mathrm{~m}$ ( 5 to $10 \mathrm{ft}$ ) of the anchor bond zone; any 


\begin{tabular}{|c|c|c|}
\hline \multicolumn{3}{|c|}{$\begin{array}{l}\text { Table } 8.2 \\
\text { Presumptive Average Ultimate Bond Stress for Ground/Grout Along } \\
\text { Anchor Bond Zone (after Table } 7 \text { of Sabatini, Pass, and Bachus } \\
\text { 1999) }\end{array}$} \\
\hline \multicolumn{3}{|c|}{$\begin{array}{ll}\text { a. Rock } \\
\end{array}$} \\
\hline \multirow[b]{2}{*}{ Rock Type } & \multicolumn{2}{|c|}{ Average Ultimate Bond Stress } \\
\hline & $\mathrm{MPa}$ & psi \\
\hline Granite and basalt & $1.7-3.1$ & $250-450$ \\
\hline Dolomitic limestone & $1.4-2.1$ & $200-300$ \\
\hline Soft limestone & $1.0-1.4$ & $150-200$ \\
\hline Slates and hard shales & $0.8-1.4$ & $120-200$ \\
\hline Soft shales & $0.2-0.8$ & $30-120$ \\
\hline Sandstones & $0.8-1.7$ & $120-250$ \\
\hline Weathered sandstones & $0.7-0.8$ & $100-120$ \\
\hline Chalk & $0.2-1.1$ & $30-160$ \\
\hline Weathered marl & $0.15-0.25$ & $20-40$ \\
\hline Concrete & $1.4-2.8$ & $200-400$ \\
\hline \multicolumn{3}{|c|}{ b. Cohesive Soil } \\
\hline \multirow[b]{2}{*}{ Anchor Type } & \multicolumn{2}{|c|}{ Average Ultimate Bond Stress } \\
\hline & $\mathrm{MPa}$ & psi \\
\hline Gravity-grouted anchors (straight shaft) & $0.03-0.07$ & $4-10$ \\
\hline \multicolumn{3}{|l|}{ Pressure-grouted anchors (straight shaft) } \\
\hline Soft silty clay & $0.03-0.07$ & $4-10$ \\
\hline Silty clay & $0.03-0.07$ & $4-10$ \\
\hline Stiff clay (med. to high plasticity) & $0.03-0.10$ & $4-15$ \\
\hline Very stiff clay (med. to high plasticity) & $0.07-0.17$ & $10-25$ \\
\hline Stiff clay (med. plasticity) & $0.10-0.25$ & $15-35$ \\
\hline Very stiff clay (med. plasticity) & $0.10-0.35$ & $15-50$ \\
\hline Very stiff sandy silt (med. plasticity) & $0.28-0.38$ & $40-55$ \\
\hline \multicolumn{3}{|c|}{ c. Cohesionless Soil } \\
\hline \multirow[b]{2}{*}{ Anchor Type } & \multicolumn{2}{|c|}{ Average Ultimate Bond Stress } \\
\hline & $\mathrm{MPa}$ & psi \\
\hline Gravity-grouted anchors (straight shaft) & $0.07-0.14$ & $10-20$ \\
\hline \multicolumn{3}{|l|}{ Pressure-grouted anchors (straight shaft) } \\
\hline Fine-med. sand (med. dense-dense) & $0.08-0.38$ & $12-55$ \\
\hline Med.-coarse sand, w/gravel (med. dense) & $0.11-0.66$ & $16-96$ \\
\hline Med.-coarse sand, w/gravel (dense-very dense) & $0.25-0.97$ & $36-140$ \\
\hline Silty sands & $0.17-0.41$ & $25-60$ \\
\hline Dense glacial till & $0.30-0.52$ & $44-75$ \\
\hline Sandy gravel (med. dense-dense) & $0.21-1.38$ & $30-200$ \\
\hline Sandy gravel (dense-very dense) & $0.28-1.38$ & $40-200$ \\
\hline
\end{tabular}

additional length of anchor bond zone may be considered to provide the additional margin of safety. Therefore, use of average bond stress values such as those provided in Table 8.2a may result in a calculated bond length significantly greater than that required to resist the design load. 


\begin{tabular}{|c|c|c|}
\hline \multicolumn{3}{|c|}{$\begin{array}{l}\text { Table } 8.3 \\
\text { Presumptive Ultimate Values of Load Transfer for Preliminary } \\
\text { Design of Ground Anchors in Rock (after Table } 8 \text { of Sabatini, Pass, } \\
\text { and Bachus 1999) }\end{array}$} \\
\hline \multirow[b]{2}{*}{ Rock Type } & \multicolumn{2}{|c|}{$\begin{array}{ll}\text { Estimated Ultimate Transfer Load } \\
\end{array}$} \\
\hline & $\mathrm{kN} / \mathrm{m}$ & kips/ft \\
\hline Granite of basalt & 730 & 50 \\
\hline Dolomitic limestone & 580 & 40 \\
\hline Soft limestone & 440 & 30 \\
\hline Sandstones & 440 & 30 \\
\hline Slates and hard shales & 360 & 25 \\
\hline Soft shales & 150 & 10 \\
\hline
\end{tabular}

\subsubsection{Predesign and production load testing}

Predesign load tests are occasionally performed to evaluate ultimate anchor load-carrying capacity and/or creep behavior of anchors installed in creepsusceptible soils. When the capacity of the individual anchors is critical to the design, it may be desirable to install and test several test anchors. Predesign load tests may be performed for cases where the required capacity of the anchors exceeds local experience, or where the required construction method is unusual. In general, predesign load tests are not commonly used and, when they are conducted, they are usually performed as part of a separate contract that is paid for by the owner.

Anchors used for predesign load tests are generally not incorporated into the final structure as load-carrying elements because of the damage that may be induced by the high testing loads required to evaluate ultimate anchor capacity. If possible, the anchors should be fabricated and installed exactly as planned for the production anchors. If testing will exceed 80 percent of the guaranteed ultimate tensile strength (GUTS) of the production anchors, additional tendon capacity should be provided. (That is, increase the number of strands or use larger bar diameter.) The procedure used for a predesign testing program can be found in Appendix D of Sabatini, Pass, and Bachus (1999). The objective of most predesign test programs is to establish the anchor load at which the creep rate becomes unacceptable. Complete documentation of a predesign test program for the I-90 project in Seattle, WA, is contained in Cheney (1990). In general, predesign load testing test programs are rarely executed due to time and cost factors.

Preproduction anchor testing programs, which can provide similar information concerning acceptable anchor loads, are commonly performed. With a preproduction testing program, the contractor performs performance tests on several anchors. Performance tests involve incremental loading and unloading to a maximum test load equal to 133 percent of the design load. Extended creep tests are commonly used in the preproduction testing program to evaluate the creep behavior of the anchor at all test loads from 25 to 133 percent of the design load. The advantages of preproduction load testing, as compared to predesign load testing, include these: (1) less expensive since contractor mobilizes to site on only one occasion, (2) less time consuming (e.g., 1 day), and (3) ability to duplicate 
ground conditions for production anchors. The results of the early-on performance tests carried out as part of a preproduction load testing program may be used to verify anchor bond zone load transfer rates, or as a means to optimize wall design through the use of a higher load transfer rate as compared to the load transfer rate used to develop the original design.

\subsubsection{Spacing requirements for ground anchors}

Each ground anchor in an anchored system is commonly designed assuming that the anchor carries a tributary area of load based on the horizontal and vertical spacing between adjacent anchors. The size and strength of the anchor tendon, drilling and grouting procedures, and diameter and length of the anchor are selected to ensure that the ground anchor can carry this load throughout its service life. The horizontal and vertical spacing of the ground anchors will vary depending on project-specific requirements and constraints, which may include (1) necessity for a very stiff system (i.e., closely spaced anchors) to control lateral wall movements, (2) existing underground structures and utilities that may affect the positioning and inclination of the anchors, and (3) type of vertical wall elements selected for the design.

The vertical position of the uppermost ground anchor (i.e., the ground anchor closest to the ground surface) should be evaluated considering the allowable cantilever deformations of the wall. The vertical position of the uppermost anchor must also be selected to minimize the potential for exceeding the passive capacity of the retained soil during proof and performance testing. During load testing, permanent anchors are typically loaded to 133 percent of the design load, resulting in movement of the wall into the retained ground. If the design load for the uppermost ground anchor is relatively large, as is the case where large surcharge loads must be resisted, or of the soils are disturbed or relatively weak, the passive capacity of the soil may be exceeded during testing. If the passive capacity is exceeded, the soldier beams or sheet pile will move excessively into the ground; for soldier beam wall systems, the timber lagging may bend and crack excessively. The passive capacity of the soldier beam required to resist the test load applied to the upper ground anchor may be calculated using Equation 8.4 (Weatherby 1998a). For this calculation, it is assumed that the passive resistance, $F_{p}$, will be developed over a depth of 1.5 times the distance to the upper ground anchor.

$$
F_{p}=1.125 K_{p} \gamma h_{1}^{2} s
$$

where

$$
\begin{aligned}
K_{p} & =\text { passive earth pressure coefficient } \\
\gamma & =\text { total unit weight of soil } \\
h_{1} & =\text { distance from surface to uppermost ground anchor } \\
s & =\text { soldier beam spacing }
\end{aligned}
$$


The passive earth pressure coefficient $\left(K_{p}\right)$ can be determined using Rankine, Coulomb, or the log-spiral method. In using Equation 8.4, a factor of safety of 1.5 is applied to the maximum capacity to obtain the allowable resistance. The allowable resistance should be greater than the upper ground anchor test load.

For ground anchors installed in soil, a minimum overburden of $4.5 \mathrm{~m} \mathrm{(15 \textrm {ft } )}$ over the center of the anchor bond zone is required (Figure 8.5). This is needed to prevent grout leakage during the installation of pressure-grouted anchors and to prevent heave at the ground surface resulting from large grouting pressures. For gravity-grouted anchors, the minimum overburden criterion is required to provide the necessary soil overburden pressure to develop anchor capacity.

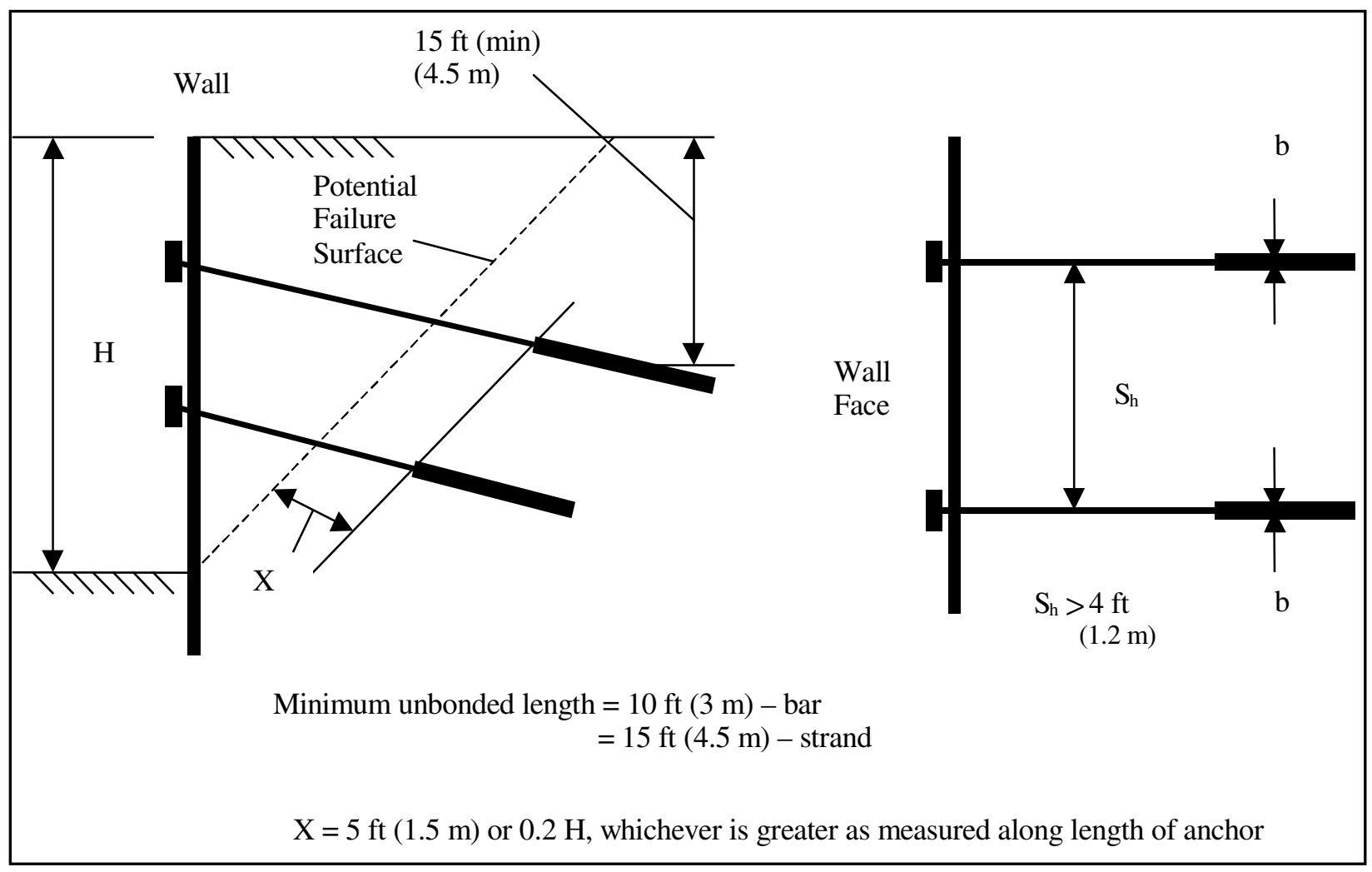

Figure 8.5. Vertical and horizontal spacing requirements for ground anchors

The maximum allowable spacing between anchors is based on allowable individual ground anchor loads and flexural capacity of individual soldier beams or sheet-pile sections. Typical horizontal spacing for soldier beams is 1.5 to $3 \mathrm{~m}$

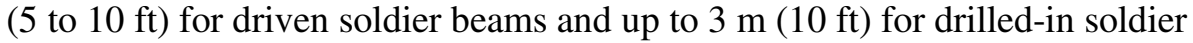
beams. The minimum horizontal spacing between anchors indicated in Figure 8.5 ensures that group effects between adjacent ground anchors are minimized and that anchor intersection due to drilling deviations is avoided. Group effects reduce the load-carrying capacity of individual ground anchors. 


\subsubsection{Selection of prestressing steel element}

The prestressing steel element of the tendon (i.e., strand or bar) must be capable of safely transmitting load in the anchor bond zone to the structure without tendon rupture. For the design load and the lock-off load, separate factors of safety are applied with respect to the potential failure mechanism of tendon rupture. The design load shall not exceed 60 percent of the specified minimum tensile strength (GUTS) of the prestressing steel. The lock-off load shall not exceed 70 percent of the GUTS, and the maximum load shall not exceed 80 percent of the GUTS.

For example, if the maximum test load is 133 percent of the design load, then the ground anchor should be selected based on a maximum allowable design load of $(0.8 / 1.33)$ GUTS, or 0.60 GUTS. If the maximum test load is 150 percent of the design load, the ground anchor should be selected based on a maximum allowable design load of (0.8/1.50) GUTS, or 0.53 GUTS.

Dimensions and strengths of bars and strands commonly used in the United States are provided in Tables 8.4 and 8.5, respectively. Larger size strand tendons (i.e., strand tendons with more strands than shown in Table 8.5) are available for applications requiring high-capacity anchorage systems. Information on 0.5 -in.(13-mm-) diameter strand can be found in ASTM A416.

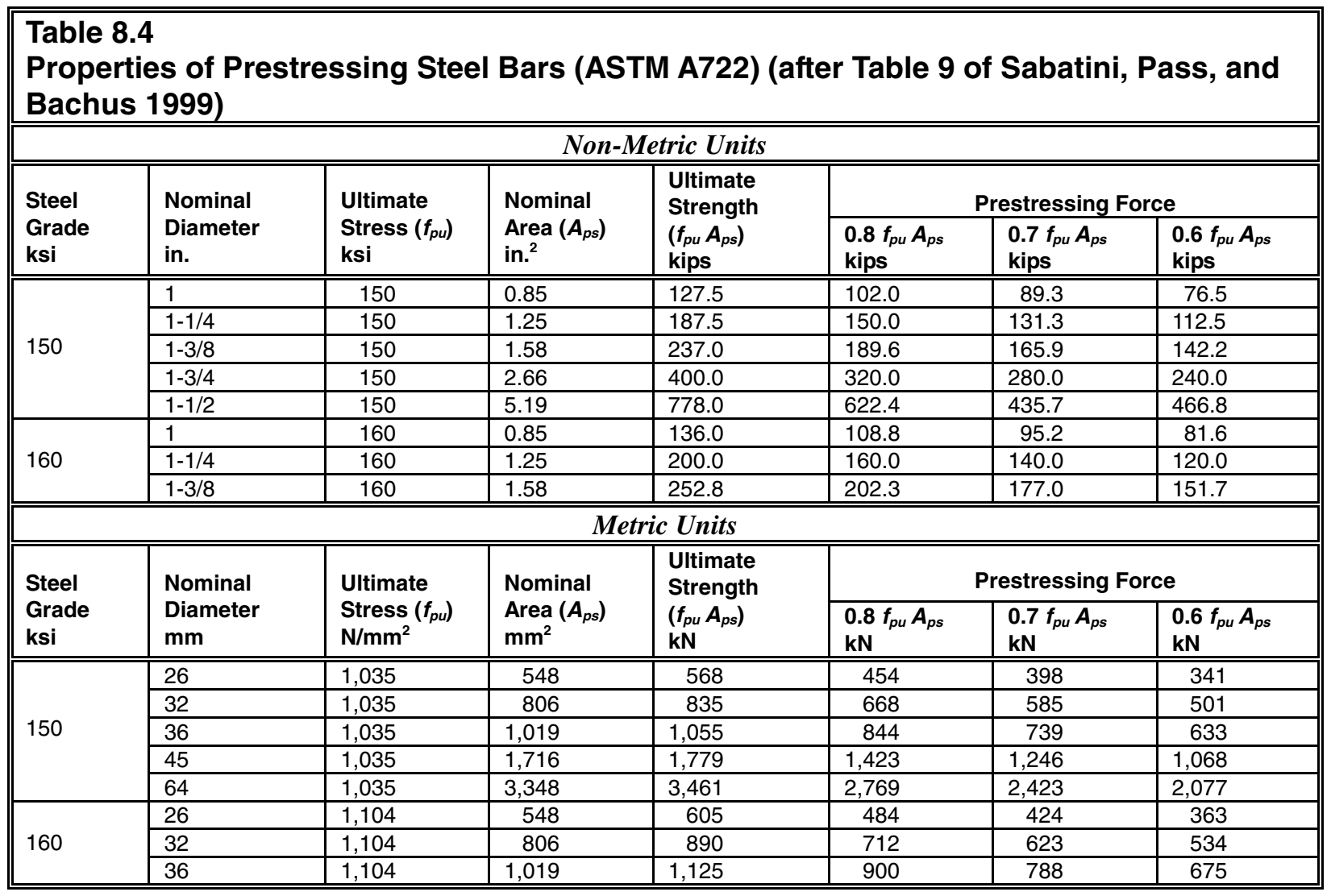




\begin{tabular}{|c|c|c|c|c|c|}
\hline \multicolumn{6}{|c|}{$\begin{array}{l}\text { Table } 8.5 \\
\text { Properties of Prestressing Steel Strands (ASTM A416, Grade 270) } \\
\text { (after Table } 10 \text { of Sabatini, Pass, and Bachus 1999) }\end{array}$} \\
\hline \multicolumn{6}{|c|}{ Non-Metric Units } \\
\hline \multirow{2}{*}{$\begin{array}{l}\text { Number of } \\
0.6 \text {-in.-diam } \\
\text { Strands }\end{array}$} & \multirow[b]{2}{*}{$\begin{array}{l}\text { Area } \\
\text { in. }^{2}\end{array}$} & \multirow{2}{*}{$\begin{array}{l}\text { Ultimate } \\
\text { Strength } \\
\text { kips }\end{array}$} & \multicolumn{3}{|c|}{$\begin{array}{c}\text { Prestressing Force } \\
\end{array}$} \\
\hline & & & $\begin{array}{l}0.8 f_{p u} A_{p s} \\
\text { kips }\end{array}$ & $\begin{array}{l}0.7 f_{p u} A_{p s} \\
\text { kips }\end{array}$ & $\begin{array}{l}0.6 f_{p u} A_{p s} \\
\text { kips }\end{array}$ \\
\hline $\begin{array}{ll}1 \\
\end{array}$ & 0.217 & $\begin{array}{l}58.6 \\
\end{array}$ & $=46.9$ & $\begin{array}{l}41.0 \\
\end{array}$ & $3 \overline{35.2}$ \\
\hline 3 & 0.651 & 175.8 & 140.6 & 123.1 & 105.5 \\
\hline 4 & 0.868 & 234.4 & 187.5 & 164.1 & 140.6 \\
\hline 5 & 1.085 & 293.0 & 234.4 & 205.1 & 175.8 \\
\hline 7 & 1.519 & 410.2 & 328.2 & 287.1 & 246.1 \\
\hline 9 & 1.953 & 527.4 & 421.9 & 369.2 & 316.4 \\
\hline 12 & 2.604 & 703.2 & 562.6 & 492.2 & 421.9 \\
\hline 15 & 3.255 & 879.0 & 703.2 & 615.3 & 527.4 \\
\hline 19 & 4.123 & $1,113.4$ & 890.7 & 779.4 & 668.0 \\
\hline \multicolumn{6}{|c|}{ Metric Units } \\
\hline \multirow{2}{*}{$\begin{array}{l}\text { Number of } \\
0.6 \text {-in.-diam } \\
\text { Strands }\end{array}$} & \multirow[b]{2}{*}{$\begin{array}{l}\text { Area } \\
\mathbf{m m}^{2} \\
\end{array}$} & \multirow{2}{*}{$\begin{array}{l}\text { Ultimate } \\
\text { Strength } \\
\text { kN }\end{array}$} & \multicolumn{3}{|c|}{ Prestressing Force } \\
\hline & & & $\begin{array}{l}0.8 f_{p u} A_{p s} \\
\mathrm{kN}\end{array}$ & $\begin{array}{l}0.7 f_{p u} A_{p s} \\
\mathrm{kN}\end{array}$ & $\begin{array}{l}0.6 f_{p u} A_{p s} \\
\mathrm{kN}\end{array}$ \\
\hline 10 & 14140 & 20260.7 & 209 & $=182$ & 156 \\
\hline 3 & 420 & 782.1 & 626 & 547 & 469 \\
\hline 4 & 560 & 1,043 & 834 & 730 & 626 \\
\hline 5 & 700 & 1,304 & 1,043 & 912 & 782 \\
\hline 7 & 980 & 1,825 & 1,460 & 1,277 & 1,095 \\
\hline 9 & 1,260 & 2,346 & 1,877 & 1,642 & 1,408 \\
\hline 12 & 1,680 & 3,128 & 2,503 & 2,190 & 1,877 \\
\hline 15 & 2,100 & 3,911 & 3,128 & 2,737 & 2,346 \\
\hline 19 & 2,660 & 4,953 & 3,963 & 3,467 & 2,972 \\
\hline
\end{tabular}

The type and size of the anchors should be evaluated prior to design of the anchor bond zone because the required hole diameter varies as a function of the tendon size. Table 8.6 can be used to estimate the minimum trumpet opening for strand or bar tendons.

\begin{tabular}{|c|c|c|c|c|c|}
\hline $\begin{array}{l}\text { Table } \\
\text { Guid } \\
\text { Oper }\end{array}$ & $\begin{array}{l}\text { on } \\
\text { Size }\end{array}$ & $\begin{array}{l}\text { latio } \\
\text { Tabl }\end{array}$ & $\begin{array}{l}\text { etween Te } \\
\text { Sabatini, P }\end{array}$ & $\begin{array}{l}\text { on } \mathrm{S} \\
\mathrm{S} \text {, an }\end{array}$ & $\begin{array}{l}\text { Trumpet } \\
\text { Is 1999) }\end{array}$ \\
\hline & ר Type & & num Suggestec & rumpe & Size \\
\hline No. of & is & Clas & ion Protection & Clas & ion Protection \\
\hline & & in. & $\mathrm{mm}$ & in. & $\mathrm{mm}$ \\
\hline 4 & & $\begin{array}{l}4 \\
\end{array}$ & 102 & $5-7 / 8$ & 150 \\
\hline 7 & & $4-1 / 2$ & 115 & $6-1 / 2$ & 165 \\
\hline 9 & & 5 & 127 & 7 & 178 \\
\hline 11 & & $5-1 / 2$ & 140 & $7-1 / 2$ & 191 \\
\hline 13 & & 6 & 153 & 8 & 203 \\
\hline 17 & & $6-1 / 2$ & 165 & $8-1 / 2$ & 216 \\
\hline & ameter & & & & \\
\hline in. & $\mathrm{mm}$ & & & & \\
\hline 1 & 26 & $2-1 / 2$ & 64 & $3-1 / 2$ & 89 \\
\hline $1-1 / 4$ & 32 & $2-7 / 8$ & 70 & 3-3/4 & 95 \\
\hline $1-3 / 8$ & 36 & 3 & 76 & 4 in. & 102 \\
\hline
\end{tabular}




\subsection{Wall Design-General}

Tieback wall systems are designed to resist lateral loads resulting from apparent pressure envelopes or conventional (Rankine) earth pressure diagrams including appropriate surcharges, water forces, and seismic forces. Loads on tieback wall systems are described in Chapter 5. (Figures 5.3 and 5.4 provide the apparent pressures (per foot of wall) recommended for use in the design of flexible tieback wall systems with single and multiple rows of anchors, respectively.)

The equations that are provided in Chapter 5 are based on the tributary area method and are those often recommended to be used to calculate bending moments in the tieback wall lateral load-resisting system. For the discrete wall systems (soldier beam with wood lagging, secant cylinder pile system, and the slurry wall system consisting of soldier beams with concrete lagging), it is assumed that the soldier beams span vertically between supports. This assumption applies to continuous sheet-pile wall systems and generally to the continuous reinforced concrete slurry wall systems, although in the latter instance some benefits can be obtained through 2-D plate action if reinforcing steel is provided in both directions. In the case of discrete wall systems, the moments determined in accordance with Chapter 5 must be multiplied by the soldier beam spacing to obtain the total apparent pressure moment demand on the soldier beam. For the continuous wall systems, the moments determined in accordance with Chapter 5 can be used directly to determine the wall moment demands on a per unit width basis. For flexible wall systems, where the total load is used to construct the apparent pressure diagram, the selection of an appropriate member section is based on the maximum bending moment calculated per the equations of Chapter 5.

In general, the same approach can be used where stiffer wall systems are needed to meet displacement performance objectives, provided the total load is based on at-rest or "near at-rest" pressure conditions, and provided both apparent (trapezoidal) and triangular pressure distributions are considered in the analysis. For walls constructed in competent soils such as most sands and stiff clays, the maximum moment will occur in the exposed section of the tieback wall and can be determined as described above. For walls that penetrate deep deposits of weak material, the maximum bending moment may occur in the embedded section of the wall. The embedded section of the wall refers to the portion of the wall that is below the final excavation grade. Bending in this section of the wall is discussed in Section 8.7.

The design of flexible tieback wall systems such as sheet-pile systems and soldier beam and lagging systems, when such systems are in relatively uniform competent material, is most often based on apparent earth pressures using an equivalent beam on rigid supports (RIGID) analysis. With such an analysis it is usually only necessary to check the final stage of construction provided that (1) the ground can develop adequate passive resistance below the excavation to support the wall, (2) apparent earth pressure diagrams have been used to assess the loading on the wall, and (3) there is minimal overexcavation below each anchor level. For cases in which there are large concentrated surcharges or berms at the ground surface, it is prudent to check wall bending moments for the initial 
cantilever stage (i.e., stage just prior to installation and lock-off of the uppermost anchor).

Often with the stiffer wall systems, the final excavation height is not the most critical condition, and in such cases designers commonly use a staged construction analysis where the maximum wall bending moment, wall deflections, and wall embedment depth are evaluated for several stages of construction. Staged excavation analysis is required since the maximum bending moment may occur at an intermediate stage of construction (i.e., before the final excavation is reached). For both flexible and stiff wall systems, intermediate construction stages can be critical. This occurs when (1) triangular earth pressure diagrams are used to design the wall; (2) the excavation extends significantly below an anchor level prior to stressing that anchor; (3) a cutoff wall is used to maintain the water level behind the wall; (4) the soil below the bottom of the wall is weak, resulting in active earth pressures that are greater than available resistance provided by the toe of the wall; (5) structures are located near the wall; and (6) displacement control is a performance objective.

\subsection{Discrete Wall Systems}

\subsubsection{General}

Discrete wall systems are the soldier beam and timber lagging systems, the secant cylinder pile systems, and the concrete slurry soldier beam and lagging systems described in Chapter 2. The following sections describe design approaches related to the various structural components of these systems.

\subsubsection{Allowable stress design (ASD) for steel soldier beams}

For permanent walls and temporary walls that are considered to be critical, the allowable bending stress in steel soldier beams, $F_{b}$, is in accordance with American Institutes of Steel Construction, Inc. (AISC-ASD) requirements and is equal to $0.60 F_{y}$, where $F_{y}$ is the yield stress of the steel. For soldier beams that are part of a hydraulic structure, $F_{b}$ is equal to $0.50 F_{y}$. These allowable stresses apply to Corps structures under usual loading conditions. Allowable stress increases are generally permitted for unusual and extreme loading conditions. Tieback walls used to support highways and railways should, when required, also meet AASHTO and AREA design specifications. Steel soldier beams can be either Grade $36\left(F_{y}=36 \mathrm{ksi}, 248 \mathrm{MPa}\right)$ or Grade $50\left(F_{y}=50 \mathrm{ksi}, 345 \mathrm{MPa}\right)$. For temporary support of excavation (SOE) walls, a 20-percent increase in allowable stress may be allowed for positive wall bending moments between anchor locations; no allowable stress increase is recommended for negative wall bending moments at anchor locations. The above criteria apply to both drilled and driven soldier beam systems; however, if driving conditions are difficult (stiff ground, boulders, etc.), a suitable reduction in allowable bending stress should be considered (refer to EM 1110-2-2906). 


\subsubsection{Wales and thru-beam connections}

With soldier beam systems, thru-beam connections rather than horizontal wales are often used to connect the ground anchors directly to the soldier beams. Thru-beam connections are usually fabricated before the soldier beam is driven or drilled in, as the case may be. The thru-beam connection is designed so the ground anchor load is applied at the center of the soldier beam (in line with the web in the case of steel soldier beams). Thru-beam connections in steel soldier beam construction cannot accommodate large-diameter ground anchor systems (i.e., greater than approximately $150 \mathrm{~mm}(6 \mathrm{in}$.)). Thru-beam connections are used when few ground anchor failures are anticipated. This is the case because, when a ground anchor fails, the failed anchor has to be removed from the connection and a new connection has to be fabricated. A "sidewinder" connection may be used with a replacement anchor for a temporary SOE wall, but it is not recommended for a permanent wall. A sidewinder connection is offset from the center of the soldier beam, and the ground anchor load is applied to the beam eccentric to the center line. Sidewinder connections subject the soldier beams to torsion.

For soldier beam and lagging systems, wale design will depend on the type of framing system used and on the fixity at the wale/soldier beam connection. General guidance on wale design is presented in Sabatini, Pass, and Bachus (1999). Allowable stress design is generally used with allowable stresses, as indicated below for sheet-pile wall systems.

Horizontal wales can be installed on the face of the soldier beams, or they can be recessed behind the front flange. When they are installed on the face, they can be exposed or embedded in concrete facing. If the wales remain exposed, then the ground anchor tendon corrosion protection may be exposed to the atmosphere. Therefore, it is necessary that corrosion protection for the anchorage be well designed and constructed. Since exposed wales are unattractive and must be protected from corrosion, they are not recommended for permanent anchored wall systems. Wales placed on the front face of soldier beams require thick cast-inplace concrete facing. Wales can be recessed to allow a normal-thickness concrete facing to be placed. Recessed wales must be individually fabricated, and the welding required to install them is difficult and expensive.

\subsubsection{Lagging design}

Timber lagging is most commonly used in the construction of soldier beam wall systems. However, precast concrete lagging has been used with success on Corps projects.

\subsubsection{Timber lagging for steel soldier beam systems.}

8.4.4.1.1 Installation of lagging. After installation of soldier beams, the soil in front of the wall is excavated in lifts, followed by installation of lagging. Excavation for lagging installation is commonly performed in $1.2-$ to $1.5-\mathrm{m}$ (4- to 5-ft) lifts; however, smaller lift thicknesses may be required in ground that has limited "standup" time. Lagging should be placed from the top down as soon as possible after excavation to minimize erosion of materials into the excavation. 
Prior to lagging installation, the soil face should be excavated to create a reasonably smooth contact surface for the lagging. Lagging may be placed either behind the front flange of the soldier beam or on the soldier beam. Lagging placed behind the soldier beam flanges is cut to approximate length, placed in-between the flanges of adjacent soldier beams, and secured against the soldier beam webs by driving wood wedges or shims. Lagging can also be attached to the front face of the soldier beams with clips or welded studs. In rare circumstances, lagging can be placed behind the back flange of the soldier beam. With either lagging method, gaps behind the lagging and the retained ground must be backpacked to ensure good contact.

Prior to placing subsequent lagging, a spacer (termed a "louver") is nailed to the top of the lagging board at each end of the lagging. This louver creates a gap for drainage between vertically adjacent lagging boards. The size of the gap must be sufficiently wide to permit drainage, while at the same time disallowing the retained soil to fall out from behind the boards. Typically, placing vertically adjacent lagging boards in close contact is considered unacceptable; however, some waterproofing methods may require that the gap between lagging boards be eliminated. In this case, the contractor must provide an alternate means to provide drainage.

Top-down installation of the lagging continues until the excavation reaches a

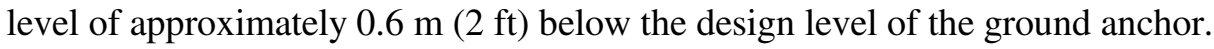
At this point, the excavation is halted and the ground anchor installed. Deeper excavation (i.e., greater than $0.6 \mathrm{~m}$ below the level of the ground anchor) may be required to allow the anchor connection to be fabricated or to provide equipment access. The wall must be designed to withstand stresses associated with a deeper excavation.

The anchor is installed using appropriate drilling and grouting procedures, as previously described. When the grout has reached an appropriate minimum strength, the anchor is load tested and then locked off at an appropriate load. Excavation and lagging installation is continued until the elevation of the next anchor is reached and the next anchor is installed. This cycle of excavation, lagging installation, and grout anchor installation is continued until the final excavation depth is reached.

8.4.4.1.2 Design of timber lagging. The thickness of temporary lagging for soldier beam and lagging walls is based primarily on experience or semiempirical rules. Lagging that is part of a permanent soldier beam and lagging system should be designed in accordance with the provisions for permanent facing systems, as outlined in Section 8.6 below. Permanent timber lagging has been used in lieu of a concrete face to carry permanent wall loads. For permanent applications, the timber grade and dimensions should be designed according to structural guidelines in accordance with the provisions for permanent facing systems. Several problems may exist for permanent timber lagging, including (1) need to provide fire protection for lagging (2) limited service life for timber, and (3) difficulty in providing corrosion protection to the ground anchor. Additional information on design of timber lagging for permanent facing is provided in Section 5.6.6 of AASHTO (1996). As previously mentioned, concrete 
lagging is not recommended due to difficulties in top-down placement of the lagging. Table 8.7 presents recommended thicknesses of construction grade lumber for temporary lagging.

\begin{tabular}{|c|c|c|c|c|c|c|c|c|c|}
\hline & & Unified & & $\operatorname{Rec}$ & commende & $\begin{array}{l}\text { d Thicknes } \\
\text { for Clear }\end{array}$ & $\begin{array}{l}\text { s of Laggir } \\
\text { Spans of: }\end{array}$ & g (Rough-C & Cut) \\
\hline & $\begin{array}{l}\text { Soil } \\
\text { Description }\end{array}$ & $\begin{array}{l}\text { Soil } \\
\text { Class. }\end{array}$ & Depth & $\begin{array}{l}5 \mathrm{ft} \\
(1.5 \mathrm{~m})\end{array}$ & \begin{tabular}{|l}
$6 \mathrm{ft}$ \\
$(1.8 \mathrm{~m})$
\end{tabular} & $\begin{array}{l}7 \mathrm{ft} \\
(2.1 \mathrm{~m})\end{array}$ & $\begin{array}{l}8 \mathrm{ft} \\
(2.4 \mathrm{~m})\end{array}$ & $\begin{array}{l}9 \mathrm{ft} \\
(2.7 \mathrm{~m}) \\
\end{array}$ & $\begin{array}{l}10 \mathrm{ft} \\
(3.0 \mathrm{~m})\end{array}$ \\
\hline Competent & $\begin{array}{l}\text { Silt or fine sand and silt } \\
\text { above water table } \\
\text { Sands and gravels } \\
\text { (medium dense to dense) }\end{array}$ & $\begin{array}{l}\mathrm{ML} \\
\mathrm{SM}-\mathrm{ML} \\
\mathrm{GW}, \mathrm{GP} \\
\mathrm{GM}, \mathrm{GS} \\
\text { SW, SP } \\
\text { SM }\end{array}$ & $\begin{array}{l}0-25 \mathrm{ft} \\
(0-8 \mathrm{~m})\end{array}$ & $\begin{array}{l}2 \mathrm{in} \\
(50 \mathrm{~mm})\end{array}$ & $\begin{array}{l}3 \mathrm{in} . \\
(75 \mathrm{~mm})\end{array}$ & $\begin{array}{l}3 \mathrm{in} . \\
(75 \mathrm{~mm})\end{array}$ & $\begin{array}{l}3 \mathrm{in} . \\
(75 \mathrm{~mm})\end{array}$ & $\begin{array}{l}4 \mathrm{in.} \\
(100 \mathrm{~mm})\end{array}$ & $\begin{array}{l}4 \text { in. } \\
(100 \mathrm{~mm})\end{array}$ \\
\hline & $\begin{array}{l}\text { Clays (stiff to very stiff) } \\
\text { nonfissured } \\
\text { Clays, medium } \\
\text { consistency } \\
\frac{\gamma H}{S_{u}} \leq 5\end{array}$ & $\begin{array}{l}\mathrm{CL}, \mathrm{CH} \\
\mathrm{CL}, \mathrm{CH}\end{array}$ & $\begin{array}{l}25-60 \mathrm{ft} \\
(8-18 \mathrm{~m})\end{array}$ & $\begin{array}{l}3 \mathrm{in} \\
(75 \mathrm{~mm})\end{array}$ & $\begin{array}{l}3 \mathrm{in} \\
(75 \mathrm{~mm})\end{array}$ & $\begin{array}{l}3 \mathrm{in} \\
(75 \mathrm{~mm})\end{array}$ & $\begin{array}{l}4 \text { in. } \\
(100 \mathrm{~mm})\end{array}$ & $\begin{array}{l}4 \mathrm{in.} \\
(100 \mathrm{~mm})\end{array}$ & $\begin{array}{l}5 \mathrm{in} . \\
(125 \mathrm{~mm})\end{array}$ \\
\hline Difficult & $\begin{array}{l}\text { Sandy and silty sand } \\
\text { (loose) } \\
\text { Clayey sands (medium } \\
\text { dense to dense) below } \\
\text { water table }\end{array}$ & $\begin{array}{l}\text { SW, SP } \\
\text { SM } \\
\text { SC }\end{array}$ & $\begin{array}{l}0-25 \mathrm{ft} \\
(0-8 \mathrm{~m})\end{array}$ & $\begin{array}{l}3 \text { in. } \\
(75 \mathrm{~mm})\end{array}$ & $\begin{array}{l}3 \mathrm{in} . \\
(75 \mathrm{~mm})\end{array}$ & $\begin{array}{l}3 \mathrm{in} . \\
(75 \mathrm{~mm})\end{array}$ & $\begin{array}{l}4 \text { in. } \\
(100 \mathrm{~mm})\end{array}$ & $\begin{array}{l}4 \mathrm{in.} \\
(100 \mathrm{~mm})\end{array}$ & $\begin{array}{l}5 \text { in. } \\
(125 \mathrm{~mm})\end{array}$ \\
\hline & $\begin{array}{l}\text { Clay, heavily over- } \\
\text { consolidated, fissured } \\
\text { Cohesionless silt, or fine } \\
\text { sand and silt below water } \\
\text { table }\end{array}$ & $\begin{array}{l}\text { CL, CH } \\
\text { ML } \\
\text { SM-SL }\end{array}$ & $\begin{array}{l}25-60 \mathrm{ft} \\
(8-18 \mathrm{~m})\end{array}$ & $\begin{array}{l}3 \mathrm{in} . \\
(75 \mathrm{~mm})\end{array}$ & $\begin{array}{l}3 \mathrm{in} . \\
(75 \mathrm{~mm})\end{array}$ & $\begin{array}{l}4 \text { in. } \\
(100 \mathrm{~mm})\end{array}$ & $\begin{array}{l}4 \mathrm{in} . \\
(100 \mathrm{~mm})\end{array}$ & $\begin{array}{l}5 \mathrm{in.} \\
(125 \mathrm{~mm})\end{array}$ & $\begin{array}{l}5 \text { in. } \\
(125 \mathrm{~mm})\end{array}$ \\
\hline Potentially & $\begin{array}{l}\text { Soft clays } \\
\frac{\gamma H}{S_{u}} \geq 5\end{array}$ & $\mathrm{CL}, \mathrm{CH}$ & $\begin{array}{l}0-16 \mathrm{ft} \\
(0-5 \mathrm{~m})\end{array}$ & $\begin{array}{l}3 \mathrm{in} . \\
(75 \mathrm{~mm})\end{array}$ & $\begin{array}{l}3 \mathrm{in} . \\
(75 \mathrm{~mm})\end{array}$ & $\begin{array}{l}4 \text { in. } \\
(100 \mathrm{~mm})\end{array}$ & $\begin{array}{l}5 \mathrm{in} . \\
(125 \mathrm{~mm})\end{array}$ & & \\
\hline $\begin{array}{l}\text { Dangerous } \\
\text { Soils }\end{array}$ & $\begin{array}{l}\text { Slightly plastic silts below } \\
\text { water table }\end{array}$ & $\mathrm{ML}$ & $\begin{array}{l}16-25 \mathrm{ft} \\
(5-8 \mathrm{~m})\end{array}$ & $\begin{array}{l}3 \mathrm{in} . \\
(75 \mathrm{~mm})\end{array}$ & $\begin{array}{l}4 \mathrm{in.} \\
(100 \mathrm{~mm})\end{array}$ & $\begin{array}{l}5 \text { in. } \\
(125 \mathrm{~mm})\end{array}$ & $\begin{array}{l}6 \text { in. } \\
(150 \mathrm{~mm})\end{array}$ & & \\
\hline & $\begin{array}{l}\text { Clayey sands (loose) } \\
\text { below water table }\end{array}$ & SC & $\begin{array}{l}25-36 \mathrm{ft} \\
(8-11 \mathrm{~m})\end{array}$ & $\begin{array}{l}4 \mathrm{in.} \\
(100 \mathrm{~mm})\end{array}$ & $\begin{array}{l}5 \text { in. } \\
(125 \mathrm{~mm})\end{array}$ & $\begin{array}{l}6 \text { in. } \\
(150 \mathrm{~mm})\end{array}$ & 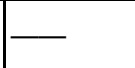 & & 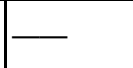 \\
\hline
\end{tabular}

\subsubsection{Concrete lagging for steel soldier beam systems.}

8.4.4.2.1 Precast concrete lagging. Precast (P/C) concrete lagging has been used, but its use may be problematic due to difficulties in handling and very tight tolerances on horizontal and vertical positioning of the soldier beam to ensure easy installation of standard length $\mathrm{P} / \mathrm{C}$ lagging. Trimming of concrete lagging is difficult, and field splicing is not possible. Also, the P/C lagging near anchor locations may crack during anchor testing or stressing. 
8.4.4.2.2 Cast-in-place concrete lagging. Cast-in-place (CIP) reinforced concrete lagging is generally designed using ultimate strength design (USD) methods in accordance with ACI 318 for the loading combinations described in Section 3.7. In most instances cast-in-place lagging is unreinforced, and as with timber lagging, the design is usually based on experience and judgment. With respect to soldier-pile-tremie-concrete systems, the concrete lagging is relatively thick so that bending and shear stresses will likely be within the limits allowed by ACI 318 for plain concrete. The costs of soldier-pile-tremie-concrete systems can be reduced substantially when reinforcing steel can be eliminated. With secant pile systems the lagging piles are unreinforced.

\subsection{Continuous Wall Systems}

\subsubsection{General}

Continuous wall systems are the sheet-pile systems and reinforced concrete slurry wall systems as described in Chapter 2. The following sections describe design approaches related to the various structural components of these systems. Additional information on wall construction procedures, materials, and equipment for other continuous wall systems is presented in FHWA-HI-99-007 (1999).

\subsubsection{Sheet-pile walls}

8.5.2.1 General. Sheet-pile walls are constructed in one phase in which interlocking sheet piles are driven to the final design elevation. Where difficult driving conditions are encountered, a template is often used to achieve proper alignment of the sheet piles. However, it should be recognized that these wall systems may not be feasible for construction in hard ground conditions, or where obstructions exist. Interlocking sheet piles may be either steel or precast concrete, although steel sheet piles are normally used because they are more readily available, and they are of higher strength than precast concrete sheet piles.

Unlike soldier beam and lagging walls, continuous walls act as both vertical and horizontal wall elements. Cycles of excavation and anchor installation proceed from the top of the excavation and then between the level of each anchor. Because of the relative continuity of these wall systems, water pressure behind continuous walls must be considered in the design. In cases where the continuous wall must resist permanent hydrostatic forces, a watertight connection must be provided at the ground anchor/wall connection.

8.5.2.2 System idealization. Sheet piles span vertically between horizontal wales. The wales are assumed to act as rigid supports for the sheetpiling. In the vertical direction the system is considered to be a continuous beam (sheet piles) on rigid support (wales). The wales act as horizontal beams carrying the sheet-pile reaction (a distributed uniform load) to tieback anchors that serve as support for the wales. Therefore, in the horizontal direction the system is also considered to be a continuous beam (wale) on rigid supports (tieback anchors). The tieback anchor load is determined based on the load transferred from the wale to the anchor. 
8.5.2.3 Allowable stress design for steel sheetpiling. For Corps structures, the allowable stresses for sheetpiling for usual load conditions are

$$
\begin{array}{ll}
\text { Combined bending and axial load } & F_{b}=0.50 F_{y} \\
\text { Shear } & F_{v}=0.33 F_{y}
\end{array}
$$

The $0.50 F_{y}$ for combined bending and axial load represents $5 / 6$ of the AISCASD recommended values and reflects the Corps ASD procedure for hydraulic steel structures. For unusual loadings the allowable stresses may be increased by 33 percent. For extreme loadings the allowable stresses may be increased by 75 percent. (See EM 1110-2-2504 for additional information on the design of sheet-pile walls.)

8.5.2.4 Allowable stress design for steel sheet wales. Horizontal wales are necessary in sheet-pile wall systems to provide support for the sheet-pile wall, which spans vertically between horizontal wale supports.

Wales that transfer the tieback forces to the sheetpiling are usually composed of back-to-back channels. From a load standpoint the most desirable position of the wales is outside the sheetpiling. The wale is considered to act as a continuous flexural member over simple supports at the tieback locations. The maximum bending moments in wales $\left(M_{M A X}\right)$ that extend over fewer than three spans is approximated as

$$
M_{M A X}=\frac{1}{8} T_{A H} S_{T}^{2}
$$

where

$$
\begin{aligned}
T_{A H} & =\text { anchor force per foot of wall } \\
S_{T} & =\text { distance between adjacent tie rods }
\end{aligned}
$$

Wales that extend over more than three supports can be considered as continuous and can be designed for a maximum bending moment $\left(M_{M A X}\right)$ equal to

$$
M_{M A X}=\frac{1}{10} T_{A H} S_{T}^{2}
$$

Sizing of the wale cross section, wale to sheetpiling connection, and tieback to wale connection is usually in accordance with AISC-ASD requirements, with the exception that, for hydraulic structures, allowable stresses are limited to 5/6 the AISC allowables. Tieback walls supporting highways or railways are designed to AASHTO and AREA allowable stress design requirements, respectively.

\subsubsection{Reinforced concrete slurry wall systems}

Continuous reinforced concrete slurry wall systems are generally designed using USD methods in accordance with ACI 318 for the loading combinations 
described in Section 3.7. These walls are generally designed for each successive stage of excavation and tieback installation, as described in Chapters 6 and 7 with respect to the Bonneville Navigation Lock temporary tieback wall. Although this construction method provides a competent structural wall system, it should be recognized that the final wall will not be to the standards and tolerances usually associated with formed wall systems. Most often, engineers will assume a 15-percent reduction in concrete strength. They will also assume that the reinforcing steel will have a light mud coating that reduces bond. Therefore, in general, designers will provide rebar splice and development lengths that are 1.5 to 2.0 times those required by ACI 318. The concrete finish obtained as a result of slurry trench construction methods may not be as desired visually with respect to the exposed sections of the wall. In such cases, cast-in-place concrete or precast concrete panel finishes are usually applied over the exposed surfaces of the slurry wall. Because the wall is continuous in both the vertical and horizontal direction, the benefits from plate bending can be considered in the analysis.

\subsection{Permanent Facing Systems}

Cast-in-place or precast facings are normally used in the construction of permanent soldier beam and lagging type walls, but can also be used with sheetpile and reinforced concrete slurry wall systems. Walls using treated timber or precast concrete lagging are not recommended as permanent facing systems since they will require considerable maintenance during their life and they are generally unattractive. Cast-in-place facings are compatible with driven or drilled-in soldier beams. Precast facings are generally used only with drilled-in soldier beam systems because of the stringent tolerance requirements needed to place and connect the precast panels. Still, the connections between the drilled-in soldier beams and the facing must be flexible enough to allow for installation tolerances. Sharply curved walls or walls with varying height generally have a cast-in-place concrete facing. Precast concrete panels allow a variety of architectural finishes to be applied to the face of the wall. Full-height, precast concrete panels are economical for long walls. Precast panels are made using reinforced concrete or prestressed concrete. Handling and lifting stresses are considered when designing tall panels. For anchored walls, permanent facing should be designed to resist earth pressures (apparent or triangular as appropriate), surcharges, water pressures, and dynamic earth pressures whenever the wall could be susceptible to earthquake ground motions. Maximum bending moments in permanent facings can be estimated using Table 8.8 .

Permanent facings that are cast-in-place are typically 200- to 300-mm (8- to 12-in.) thick. This thickness typically will ensure that the wall is structurally sound and will allow some deviations in soldier beam placement. Significant deviations in soldier beam alignment, however, may require that additional concrete in excess of that required for the nominal thickness of the wall be used so that the finished wall face is properly aligned. Precast concrete panels are designed as simple spans between soldier beams. 


\begin{tabular}{|c|c|}
\hline \multicolumn{2}{|c|}{$\begin{array}{l}\text { Table } 8.8 \\
\text { Maximum Design Bending Moments in Permanent Facing (after } \\
\text { Table } 13 \text { of Sabatini, Pass, and Bachus 1999) }\end{array}$} \\
\hline $\begin{array}{l}\text { Support and Soil Condition } \\
\end{array}$ & $\begin{array}{l}\text { Maximum Moment } \\
\text { per Foot of Height }\end{array}$ \\
\hline $\begin{array}{l}\text { Simple span. No soil arching (e.g., soft cohesive soils, rigid concrete facing } \\
\text { placed tightly against soil). }\end{array}$ & $\frac{p I^{2}}{8}$ \\
\hline $\begin{array}{l}\text { Simple span. Soil arching (e.g., granular soil or stiff cohesive soil with } \\
\text { flexible facing where space is available to allow in-place soil to arch). }\end{array}$ & $\frac{p l^{2}}{12}$ \\
\hline $\begin{array}{l}\text { Continuous facing. No soil arching (e.g., soft cohesive soils, rigid concrete } \\
\text { facing placed tightly against soil). }\end{array}$ & $\frac{p l^{2}}{10}$ \\
\hline $\begin{array}{l}\text { Continuous facing. Soil arching (e.g., granular soil or stiff cohesive soil with } \\
\text { flexible facing where space is available to allow in-place soil to arch). }\end{array}$ & $\frac{p l^{2}}{12}$ \\
\hline $\begin{array}{ll}\text { Note: } & p=\text { maximum ordinate of total pressure envelope along span. } \\
& I=\text { span between supports. }\end{array}$ & \\
\hline
\end{tabular}

\subsection{Design of Wall Toe}

\subsubsection{General}

The embedded portion of tieback wall systems must have the capacity to safely carry the lateral load resulting from the tieback wall reaction force (portion of total load not carried by anchors) plus active soil pressure, surcharge pressure, and differential water pressure acting on the embedded portion of the wall. The embedded portion of the wall must have the capacity to resist these loads with a minimum factor of safety. The factor of safety, FS, against lateral load failure is equal to the minimum ultimate lateral load resisting capacity, $F_{u l}$, divided by the tieback wall subgrade reaction force, $R$. Usually, the minimum factor of safety for lateral load resistance is 1.5 , such that

$$
F S=F_{u l t} / R \geq 1.5
$$

Methods for calculating the minimum ultimate lateral load resisting capacity of the embedded portion of the wall for continuous and discrete wall systems are described below.

Traditional methods can be used for calculating passive earth pressure resistance (Rankine, Coulomb, log-spiral) on the embedded portion of continuous-type tieback wall systems. However, the log-spiral method is recommended in Sabatini, Pass, and Bachus (1999). Additionally, Figure 3.8 of Ebeling and Morrison (1992) shows that when $\delta / \phi \geq 1 / 2$, the Coulomb solution (planer failure surface solution) should not be used to compute the passive earth pressure resistance. Passive earth pressure coefficients based on log-spiral solutions can be found in Design Manual 7.2 (NAVFAC 1982). The passive resistance for walls with discrete elements (i.e., soldier beams) below subgrade has been typically evaluated using relationships developed by Broms (1965). In cohesionless soils and for drained conditions in cohesive soils, passive resistance is assumed to be developed over three times the soldier beam width, $b$, with a magnitude determined using the Rankine passive earth pressure coefficient. In cohesive soils (e.g., soils with an undrained shear strength, $s_{u}$, that is constant with depth), the passive 
resistance is assumed to develop over one soldier beam width and to be constant over most of the beam depth with a magnitude of nine times the soil undrained shear strength. No passive resistance is assumed to develop over a depth below the surface equal to 1.5 times the soldier beam width.

Passive resistances back-calculated for soldier beam and lagging systems, however, compare more favorably with passive resistance calculations developed by Wang and Reese (1986). With the Wang and Reese equations, several different failure mechanisms must be evaluated, and the one producing the minimum resistance is selected for use in a factor of safety evaluation. The objective of most tieback toe evaluations is to satisfy minimum factor of safety requirements. Although this will satisfy collapse-prevention performance objectives, it may not satisfy displacement-control performance objectives. The latter may require embedments deeper than those determined based on minimum factor of safety requirements. The Wang and Reese equations will produce a higher passive resistance than that predicted by the Broms equations. A comparison of the two methods is presented in Sabatini, Pass, and Bachus (1999). Since the Wang and Reese equations are more representative of actual passive pressure conditions at the toe of discrete tieback wall systems, and since their use is generally recommended for the development of nonlinear soil springs for a Winkler spring analysis, the equations are presented below.

\subsubsection{Lateral capacity for discrete (soldier beam) wall systems}

Methods developed by Wang and Reese (1986) are often used to develop the ultimate passive resistance for soldier beams embedded in cohesionless and cohesive soils. The Wang-Reese equations, as they apply to cohesionless and cohesive soils, are described below. This information is taken verbatim from Appendix B of Sabatini, Pass, and Bachus (1999).

8.7.2.1 Cohesionless soils. The Wang-Reese equations for ultimate passive resistance of cohesionless soils consider three potential failure mechanisms: (1) a wedge failure in front of an individual shaft (Figure 8.6), (2) an overlapping wedge failure for deep or closely spaced shafts (Figure 8.7), and (3) plastic flow around the shaft (Figure 8.8). For design, the ultimate passive resistance available to resist the reaction force, $R$, is the minimum resistance for each of these mechanisms at any depth.

For the wedge failure in front of a single soldier beam, the passive force, $F_{p}$, is given by

$$
\begin{aligned}
F_{p}=\gamma d^{2} & {\left[\frac{K_{0} d \tan \phi^{\prime} \sin \beta}{\tan \left(\beta-\phi^{\prime}\right) \cos \alpha}+\frac{\tan \beta}{\tan \left(\beta-\phi^{\prime}\right)}\left(\frac{b}{2}+\frac{d}{3} \tan \beta \tan \alpha\right)\right.} \\
& \left.+\frac{K_{0} d \tan \beta}{3}\left(\tan \phi^{\prime} \sin \beta-\tan \alpha\right)\right]
\end{aligned}
$$


where

$\gamma=$ total unit weight

$d=$ depth of bottom of soldier beam

$K_{0}=$ at-rest pressure coefficient

$\phi^{\prime}=$ drained friction angle of the soil

$\beta=45+\phi^{\prime} / 2$

$\alpha=\phi^{\prime}$ for dense sands, $\phi^{\prime} / 3$ to $\phi^{\prime} / 2$ for loose sands

$b=$ soldier beam diameter of width

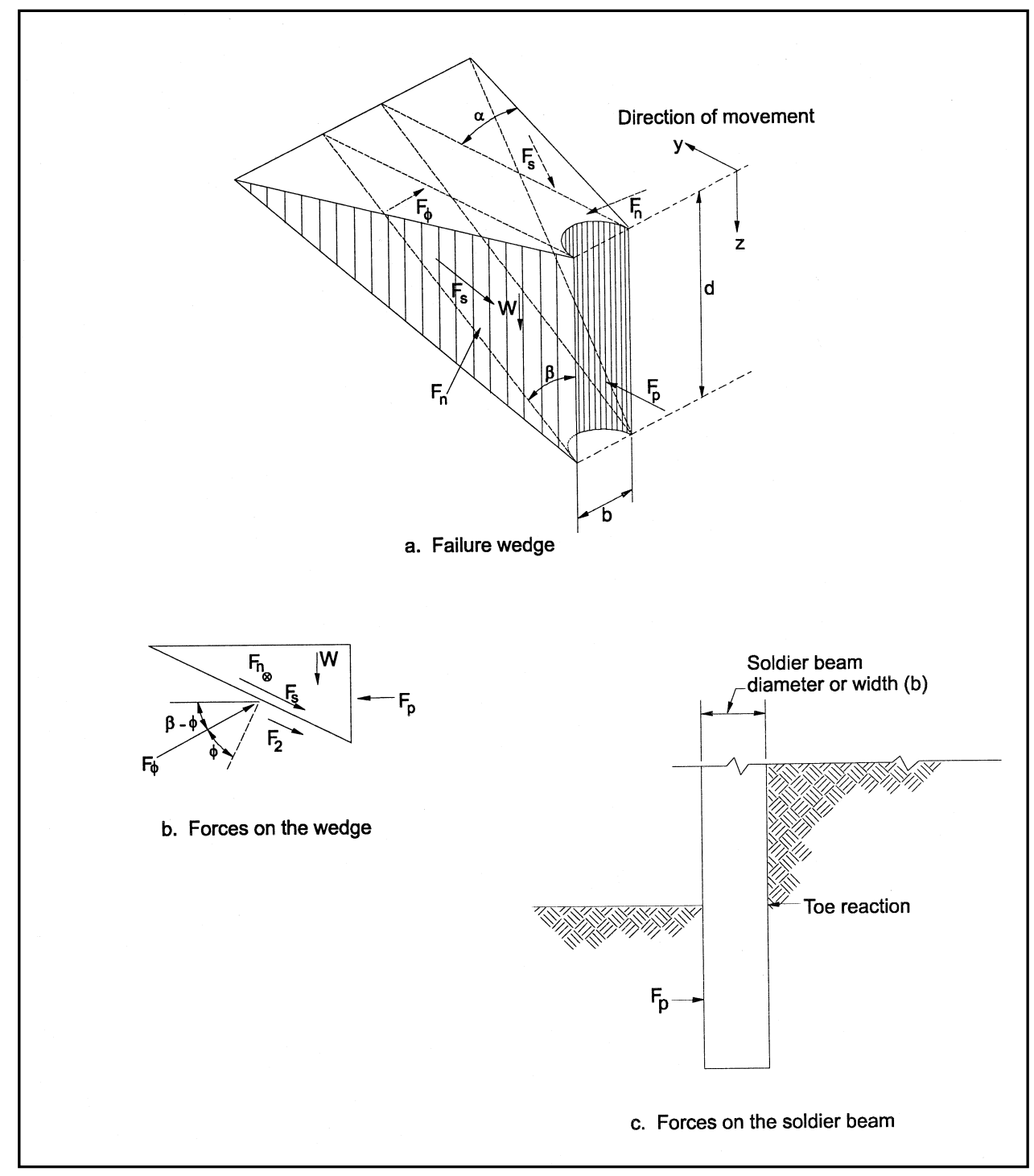

Figure 8.6. Passive wedge failure for a soldier beam in sand (after Figure B-1 of Sabatini, Pass, and Bachus 1999) 
Equation 8.7 is differentiated to give the ultimate soil resistance, $P_{p u}$, at depth $d$.

$$
\begin{aligned}
P_{p u}=\gamma d[ & \frac{K_{0} d \tan \phi^{\prime} \sin \beta}{\tan \left(\beta-\phi^{\prime}\right) \cos \alpha}+\frac{\tan \beta}{\tan \left(\beta-\phi^{\prime}\right)}(b+d \tan \beta \tan \alpha) \\
& \left.+K_{0} d \tan \beta\left(\tan \phi^{\prime} \sin \beta-\tan \alpha\right)\right]
\end{aligned}
$$

Figure 8.7 shows individual failure wedges intersecting.

The failure wedges can intersect when adjacent soldier beams are relatively close to each other or where the depth of the bottom of the soldier beam is relatively large. Equation 8.9 gives the depth of the intersection, $d_{1}$, of adjacent wedges.

$$
d_{1}=d-\frac{s_{c}}{2 \tan \alpha \tan \beta}
$$

where $s_{c}$ is the clear spacing between adjacent soldier beams.

When $d_{1}$ is positive, the failure wedges intersect. If $d_{1}$ is negative, passive resistance is not affected by adjacent soldier beams and may be computed using Equation 8.8. Above the point of intersection, passive resistance is reduced to account for the intersection of the failure wedges. To account for the intersection of the wedges, passive resistance computed using Equation 8.8 is reduced by the resistance computed for a wedge with height $d_{1}$ and a soldier beam with a width of zero. The resistance down to $d_{1}$ is given by

$$
\begin{aligned}
P_{p u}=\gamma d[ & {\left[\frac{K_{0} d \tan \phi^{\prime} \sin \beta}{\tan \left(\beta-\phi^{\prime}\right)}\left(\frac{1}{\cos \alpha}-1\right)+\frac{d \tan \beta \tan \alpha}{\tan \left(\beta-\phi^{\prime}\right)}\right.} \\
& \left.-K_{0} d \frac{\sin ^{2} \beta}{\cos \beta} \tan \phi^{\prime}(\tan \alpha+1)\right]
\end{aligned}
$$

At depth, the ultimate resistance will be limited to the resistance that can develop before soil flows between soldier beams (Figure 8.8). The ultimate flow resistance is given by

$$
P_{p u}=K_{A} b \gamma d \tan ^{8} \beta+K_{0} \gamma d \tan \phi^{\prime} \tan ^{4} \beta
$$

Lateral resistance cannot exceed the passive resistance provided by a continuous wall in cohesionless soil, that is,

$$
P_{p u}=K_{p} \gamma d\left(s_{c}+b\right)
$$




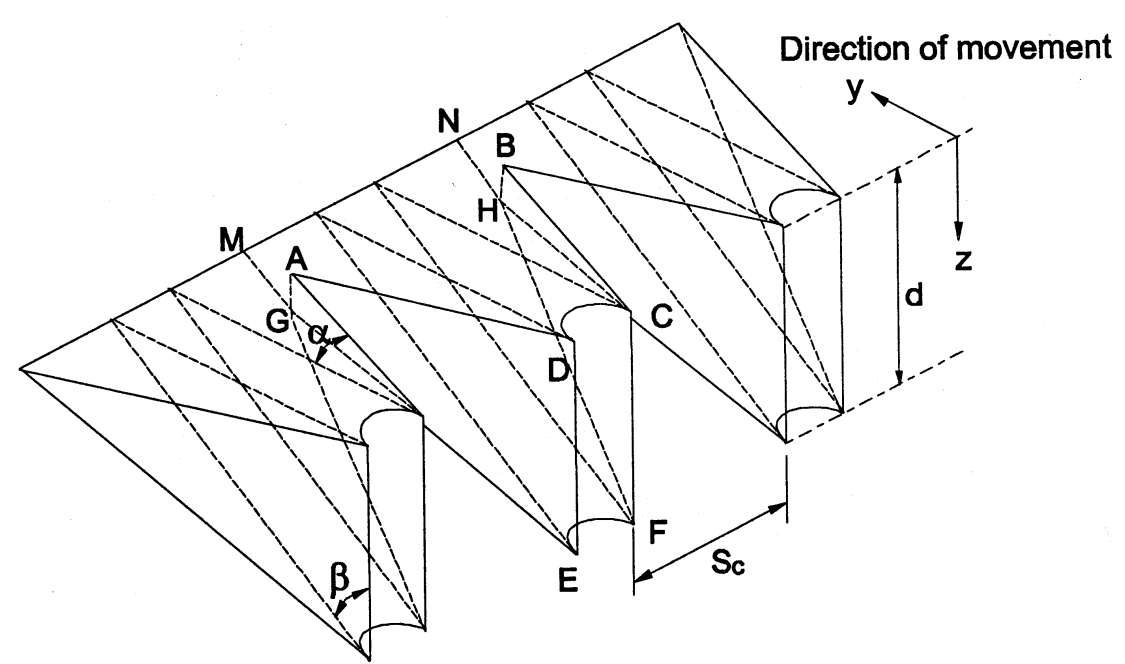

a. General view

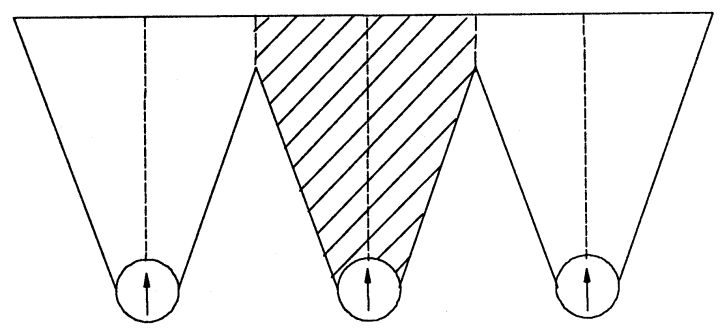

b. Plan view
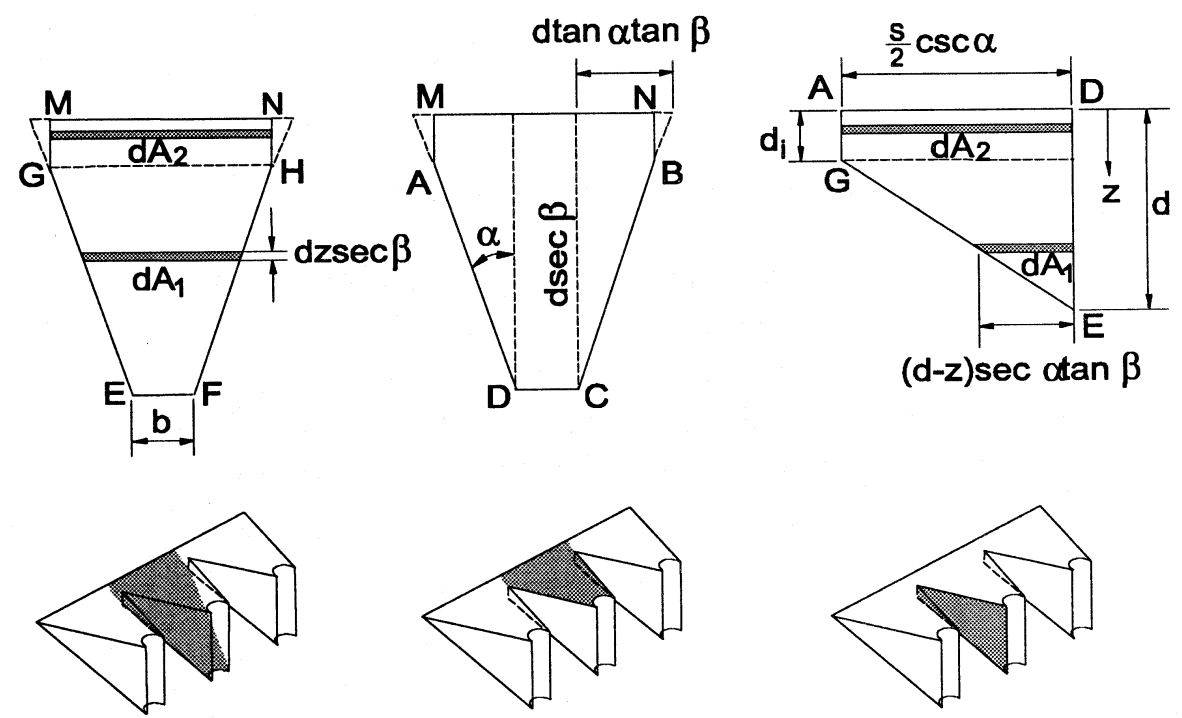

c. Bottom of a wedge

d. Top of a wedge

e. Side of a wedge

Figure 8.7. Intersecting failure wedges for soldier beams in sand (after Figure B-2 of Sabatini, Pass, and Bachus 1999) 


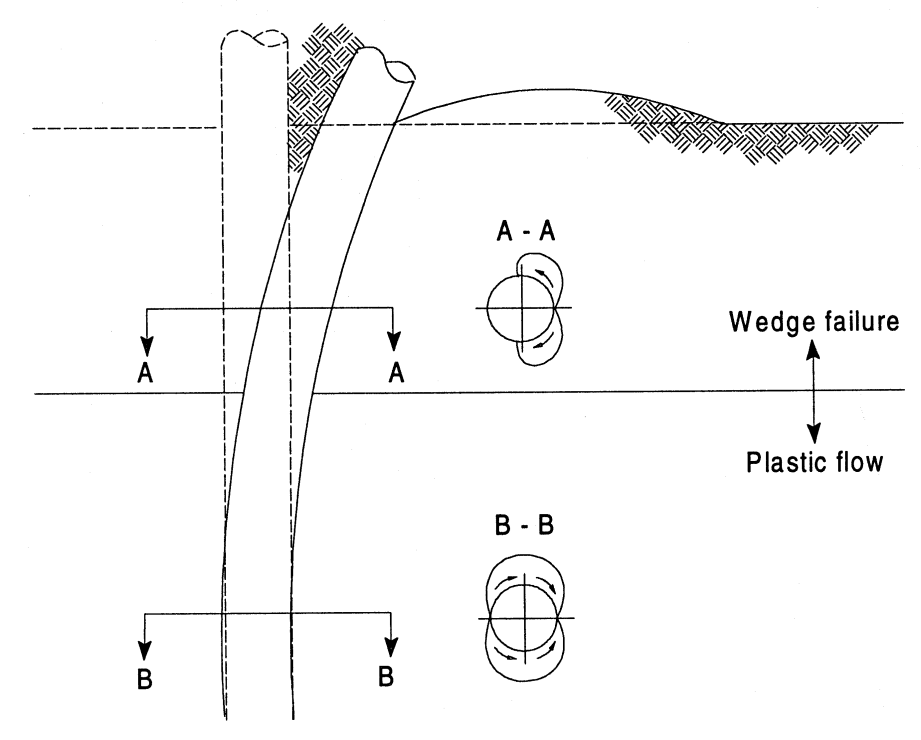

Figure 8.8. Plastic flow around a soldier beam toe (after Figure B-3 of Sabatini, Pass, and Bachus 1999)

8.7.2.1 Cohesive soils. Figure 8.9 shows the failure wedge for a single soldier beam in clay. Reese (1958) developed the expression for the passive resistance, $F_{p}$, for the failure wedge of a single soldier beam in clay.

$$
F_{p}=S_{u} d b[\tan \theta+(1+K) \cot \theta]+\frac{1}{2} \gamma b D^{2}+S_{u} D^{2} \sec \theta
$$

where

$$
\begin{aligned}
S_{u}= & \text { average undrained shear strength } \\
K= & \text { reduction factor to apply to } S_{u} \text { to give the adhesion between the clay and } \\
& \text { the soldier beam }
\end{aligned}
$$

assuming $\theta=45 \mathrm{deg}$ and the shaft friction, $K$, is equal to zero. Equation 8.12 is differentiated to give the ultimate soil resistance at depth, $d$, as

$$
P_{p u}=2 S_{u} b+\gamma b d+2.83 S_{u} d
$$

The assumption of $K=0$ implies that no shear strength is mobilized along the contact plane between the soldier beam and the clay soil.

If adjacent soldier beams are sufficiently close to each other, it may not be possible to mobilize the full shear resistance (forces $F_{3}$ and $F_{4}$ in Figure 8.9) on the sides of the failure wedge directly in front of the soldier beam. Figure 8.10 shows the passive wedges in front of each soldier beam and the wedge of soil between the beams (block FDBGHI). 


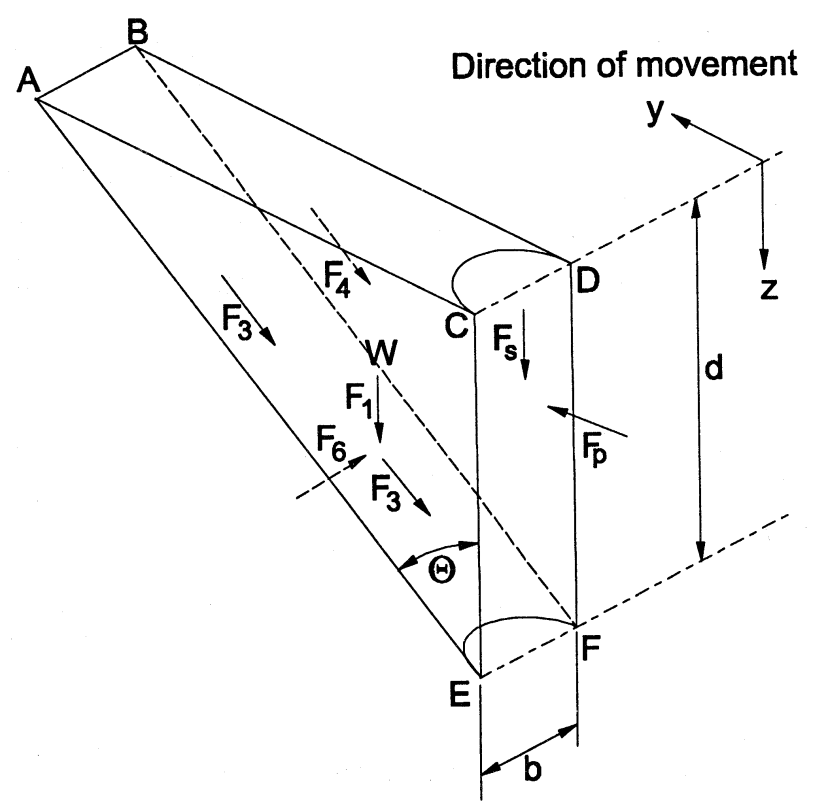

a. Failure wedge
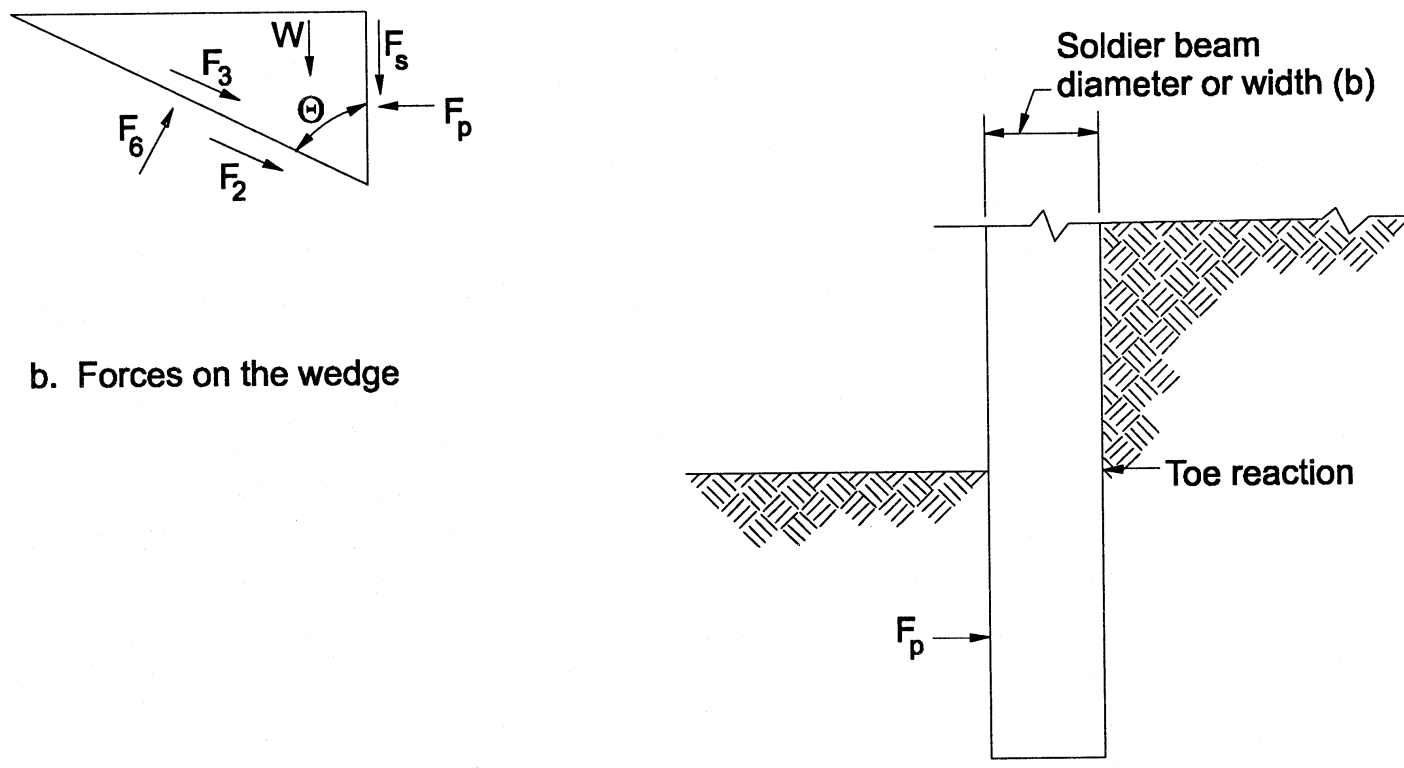

c. Forces on the soldier beam

Figure 8.9. Passive wedge failure for a soldier beam in clay (after Figure B-4 of Sabatini, Pass, and Bachus 1999) 


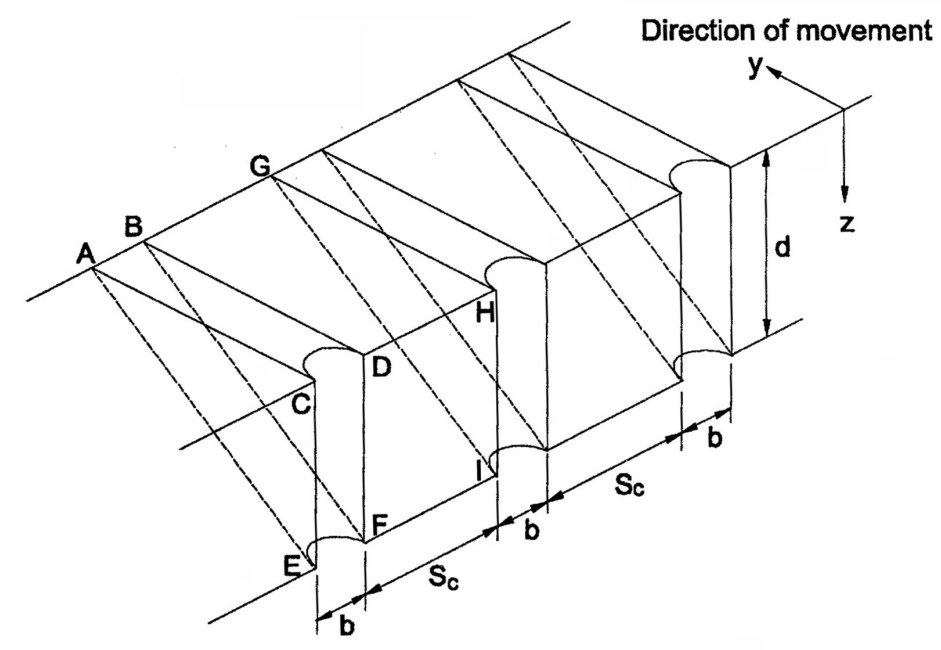

a. General view

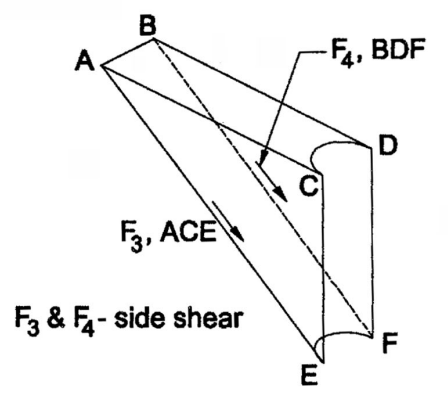

b. Wedge in front of soldier beam

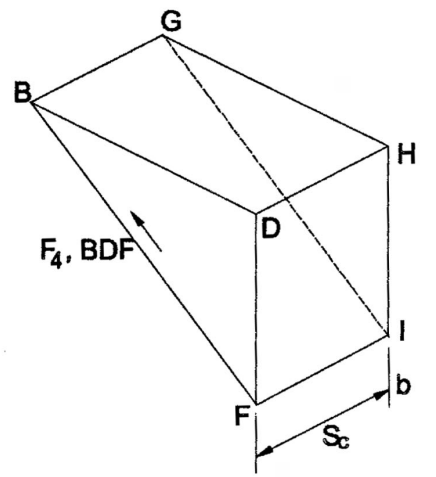

c. Block of soil between beams

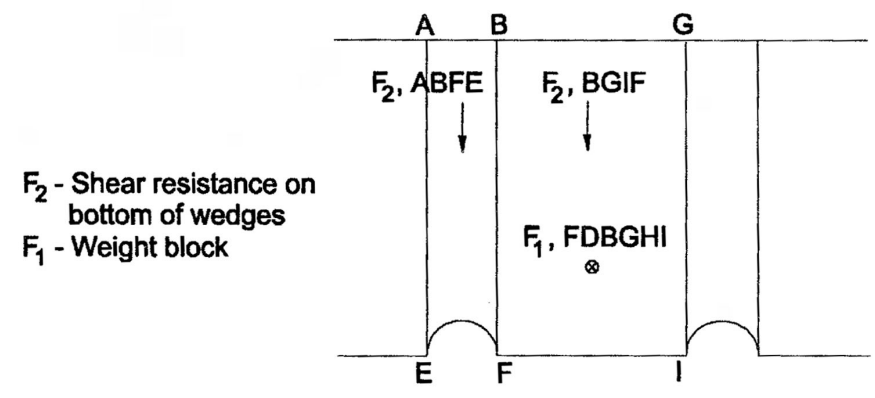

d. Plan view

Figure 8.10. Failure wedges for soldier beams in clay (after Figure B-5 of Sabatini, Pass, and Bachus 1999) 
If the spacing is small, it is assumed that the ground in front of the wall will move together and the individual wedges in front of each soldier beam will not develop. Equation 8.14 gives the critical spacing, $s_{c r}$, where the behavior changes from single soldier beam behavior to group behavior.

$$
s_{c r}=\frac{2.83 S_{u} d}{\gamma d+6 S_{u}}
$$

Passive resistance for a soldier beam considering group behavior is given by

$$
P_{p u}=2 S_{u}\left(b+s_{c}\right)+\gamma d\left(b+s_{c}\right)+S_{u} s_{c}
$$

If the spacing between soldier beams becomes zero and the soldier beam width is taken as unity, Equation 8.15 becomes Equation 8.16a, the passive earth pressure equation for a continuous wall:

$$
P_{p u}=11 S_{u}(b)
$$

The soil may flow around the beam as it moves through the soil if the toe of the soldier beam becomes sufficiently deep. The failure is similar to that shown in Figure 8.8. Wang and Reese (1986) approximated the plastic flow resistance in clay as

$$
P_{p u}=2 S_{u}+\gamma d_{c}
$$

For a wall in clay, the passive resistance at any depth $d_{1}$ cannot exceed the passive resistance provided by a continuous wall.

$$
P_{p u}=\left(2 S_{u}+\gamma d\right)\left(b+s_{c}\right)
$$

Wang and Reese's equations are based on horizontal force equilibrium. The active pressure acting on the wall as it moves away from the retained ground is included in the calculation for cohesionless soils, but not for cohesive soils. As the Wang-Reese equations were developed for stiff clays at relatively shallow depths, the active earth pressures are negative. In neglecting the active pressure term, the tensile strength of the soil is ignored.

\subsubsection{Axial capacity}

8.7.3.1 Introduction. The sum of the vertical component of each ground anchor load and other vertical loads (e.g., dead weight of wall, permanent live loads) must be considered in the design of the wall elements to minimize potential of a bearing failure and/or excessive vertical wall movement. Soldier beams for 
anchored walls are either driven or placed in predrilled holes that are subsequently backfilled with lean-mix or structural concrete. Conventional analysis of axial load capacity for driven piles and drilled shafts may be used to design the vertical wall elements of anchored walls. Analysis methods presented herein are described in Hannigan, Goble, Thendean, Likens, and Rausche (1966) for driven piles and in O'Neill and Reese (1999) for drilled piles.

8.7.3.2 Axial load evaluation. External vertical loads on anchored walls include (1) vertical ground anchor forces, (2) dead weight of wall elements (e.g., soldier beams, lagging, concrete facing), and (3) other external loads. Other loads that may be significant for anchored walls, but which are relatively difficult to evaluate a priori, include (1) load transfer to the retained ground above the subgrade and (2) downdrag loads that result when the retained ground settles relative to the wall. The method recommended herein for designing vertical wall elements of permanent walls for axial capacity assumes that all external loads are resisted by side friction and the end bearing resistance in the embedded portion of the wall. Target factors of safety for calculating the allowable axial load are suggested based on soil type.

Research results (see Weatherby 1998) and a review of the limited case history information indicate that

- In dense to very dense sands or stiff to hard clays, axial load will be partially transferred from the wall to the ground in the retained soil located above the level of excavation. The length of time is unknown as to when these loads will be finally transferred to the embedded portion of the wall.

- Axial load may be minimized by installing anchors as near to horizontal as possible.

- Downdrag loads are reduced to zero when the wall settles approximately $2.5 \mathrm{~mm}(0.01 \mathrm{in}$.) relative to the supported ground.

- Downdrag loads will likely act on walls constructed in soft to medium clays or loose to medium dense sands that are founded on a relatively firm stratum.

These observations support the conservative assumption that all external loads should be designed to be supported by the embedded portion of the wall.

Table 8.9 presents factors of safety $(F S)$ generally used for calculating the allowable axial capacity of driven and drilled-in soldier beams for permanent walls. Lower factors of safety may be justified based on the results of

\begin{tabular}{|c|c|c|}
\hline \multicolumn{3}{|c|}{$\begin{array}{l}\text { Table } 8.9 \\
\text { Recommended Factors of Safety } \\
\text { for Axial Capacity of Driven and } \\
\text { Drilled-In Soldier Beams (after } \\
\text { Table } 14 \text { of Sabatini, Pass, and } \\
\text { Bachus 1999) }\end{array}$} \\
\hline $\begin{array}{l}\text { Soil } \\
\text { Type } \\
\end{array}$ & $\begin{array}{l}\text { Factor of Safety } \\
\text { on Skin Friction }\end{array}$ & $\begin{array}{l}\text { Factor of Safety } \\
\text { on End Bearing }\end{array}$ \\
\hline Clays & 2.5 & 2.5 \\
\hline Sands & 2.0 & 2.5 \\
\hline
\end{tabular}


site-specific load testing. These factors of safety were developed based on the requirement that vertical wall movements are minimized. The allowable axial capacity, $Q_{a}$, of driven and drilled-in soldier beams is defined as

$$
Q_{a}=\frac{Q_{u l t}}{F S}
$$

Methods to calculate the ultimate axial capacity, $Q_{u l t}$, are described subsequently.

For temporary SOE applications, designs may consider the potential for axial load support above the excavation subgrade and, thus, axial capacity for SOE wall elements may be based on lower factors of safety than those listed in Table 8.9. Lower factors of safety can be used if the designer can provide data or demonstrate that vertical settlement of the wall will be relatively small. Tolerable vertical settlement is necessary to ensure that the ground anchor/wall connection does not become overstressed and that lateral movements of the wall resulting from vertical wall movement will be acceptable.

\subsubsection{Axial capacity design of driven soldier beams.}

8.7.3.3.1 Driven Soldier Beams in Sand. Relationships for estimating the axial capacity of driven soldier beams in sand were developed from procedures in Reese and O'Neill (1988). The ultimate load-carrying capacity of driven H-beams in sands is given by

$$
Q_{u l t}=f_{s} A_{s}+q A_{t}
$$

where

$$
\begin{aligned}
Q_{u l t} & =\text { ultimate pile axial capacity } \\
f_{s} & =\text { average unit skin friction resistance } \\
A_{s} & =\text { block perimeter surface area of the soldier beam toe } \\
q & =\text { unit end bearing resistance } \\
A_{t} & =\text { block area of the soldier beam tip }
\end{aligned}
$$

For piles founded on predominantly gravelly soils or rock, the actual area of the pile should be used for $A_{t}$.

The unit skin friction resistance, $f_{s}$, is calculated from the following expression:

$$
f_{s}=K \sigma_{\text {vave }}^{\prime} \tan (\delta)
$$


where

$$
\begin{aligned}
K= & \text { lateral earth pressure coefficient (recommended range, } 1 \text { to } 2 \text { ) } \\
\sigma_{\text {vave }}^{\prime}= & \text { average vertical effective stress along the toe of the soldier beam } \\
\delta= & \text { interface friction angle between the beam and the soil (recommended } \\
& \text { range, } 0.67 \phi \text { to } 0.83 \phi)
\end{aligned}
$$

If the groundwater is below the bottom of the beam, then

$$
\sigma_{\text {vave }}^{\prime}=\gamma(h+d) / 2
$$

where

$$
\begin{aligned}
\gamma & =\text { total unit weight } \\
h & =\text { height of wall } \\
d & =\text { depth of toe embedment }
\end{aligned}
$$

The effective overburden pressure in Equation 8.20 is determined using the average of the wall height plus the toe penetration. The effective overburden pressure on the side of the soldier beam toe depends upon a depth of embedment from the ground surface to the midpoint of the toe. On the other side of the soldier beam, the effective overburden pressure depends upon a depth of embedment from the bottom of the excavation to the midpoint of the toe. Lateral loads on a soldier beam toe are greater than those computed using this procedure since passive pressures develop on the excavation side of the soldier beam. Embedment depth, $d$, is used to compute the point bearing resistance, since bearing capacity is controlled by the shallow failure surface that would develop in front of the wall.

The unit end bearing resistance, $q$, can be calculated from the following expression:

$$
q=\sigma_{v}^{\prime} N_{q}
$$

where

$$
\begin{aligned}
& \sigma_{v}^{\prime}=\text { effective overburden stress at depth, } d \\
& N_{q}=\text { bearing capacity factor from Figure } 8.11
\end{aligned}
$$

To match the predictions with the capacities measured in the Texas A\&M University test wall, a lateral earth pressure coefficient $K=2$ was used. The design procedure recommends using an average friction angle, $\delta$, for steel against sand and for sand against sand. The best prediction of skin friction occurred using a value of $\delta$, equal to $0.83 \phi$. A value of $N_{q}$ in the middle range by Meyerhof gave the best estimate of end bearing capacities. 


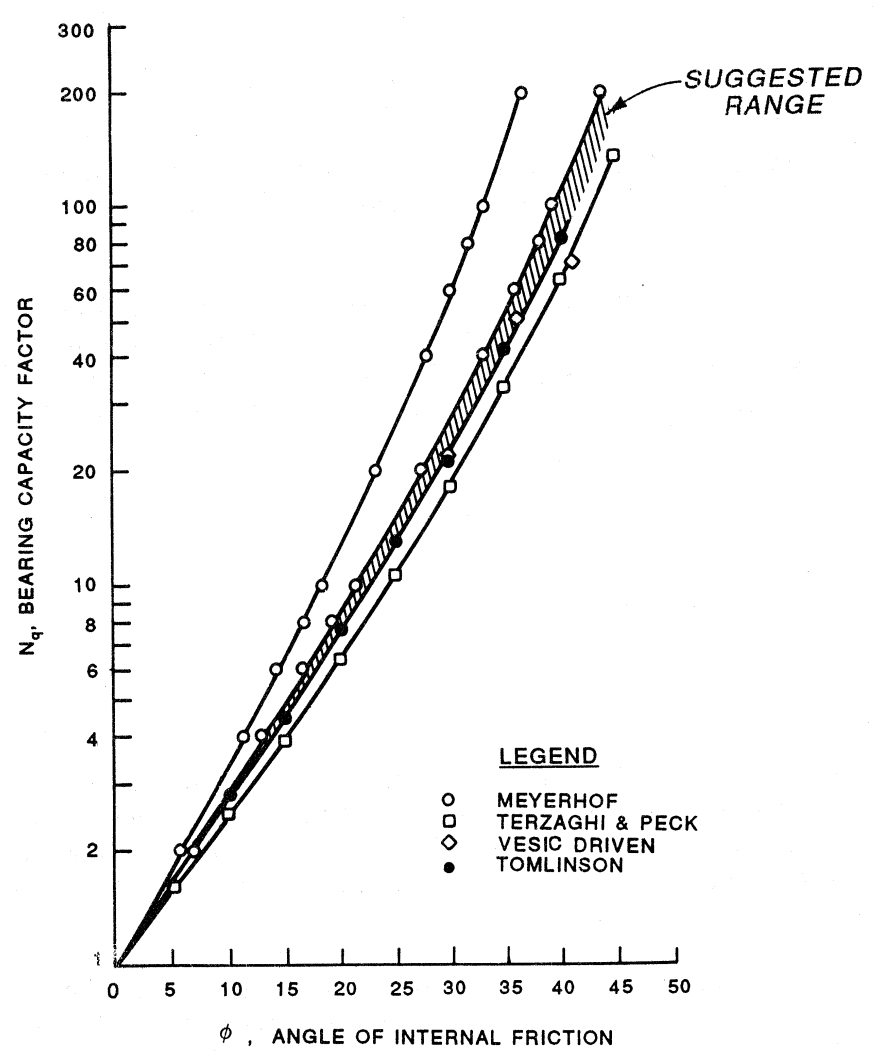

Figure 8.11. Bearing capacity factor (after Figure 4-4, EM 1110-2-2906)

The axial load carrying capacity of a drilled-in soldier beam backfilled with lean mix is estimated using Equations 8.19-8.21. These equations assume the soldier beam will punch through the backfill rather than transfer the load through the backfill to the ground. When estimating the capacity, use $K=2$ and $\delta=35 \mathrm{deg}$ in Equation 8.20. Compare the ultimate axial capacity with the capacity determined for drilled-in piles as presented in subsequent sections. Determine the toe depth using the lowest capacity.

8.7.3.3.3 Driven soldier beams in clay. The ultimate load-carrying capacity of driven H-beams in clays is given by

$$
Q_{u l t}=f_{s} A_{s}+q A_{t}
$$

The unit skin resistance, $f_{s}$, may be calculated from

$$
f_{s}=c_{a}=\alpha S_{u}
$$


where

$$
\begin{aligned}
& c_{a}=\text { adhesion between the clay and the soldier beam } \\
& \alpha=\text { adhesion factor }
\end{aligned}
$$

Values of $\alpha$ are given in Figure 8.12.

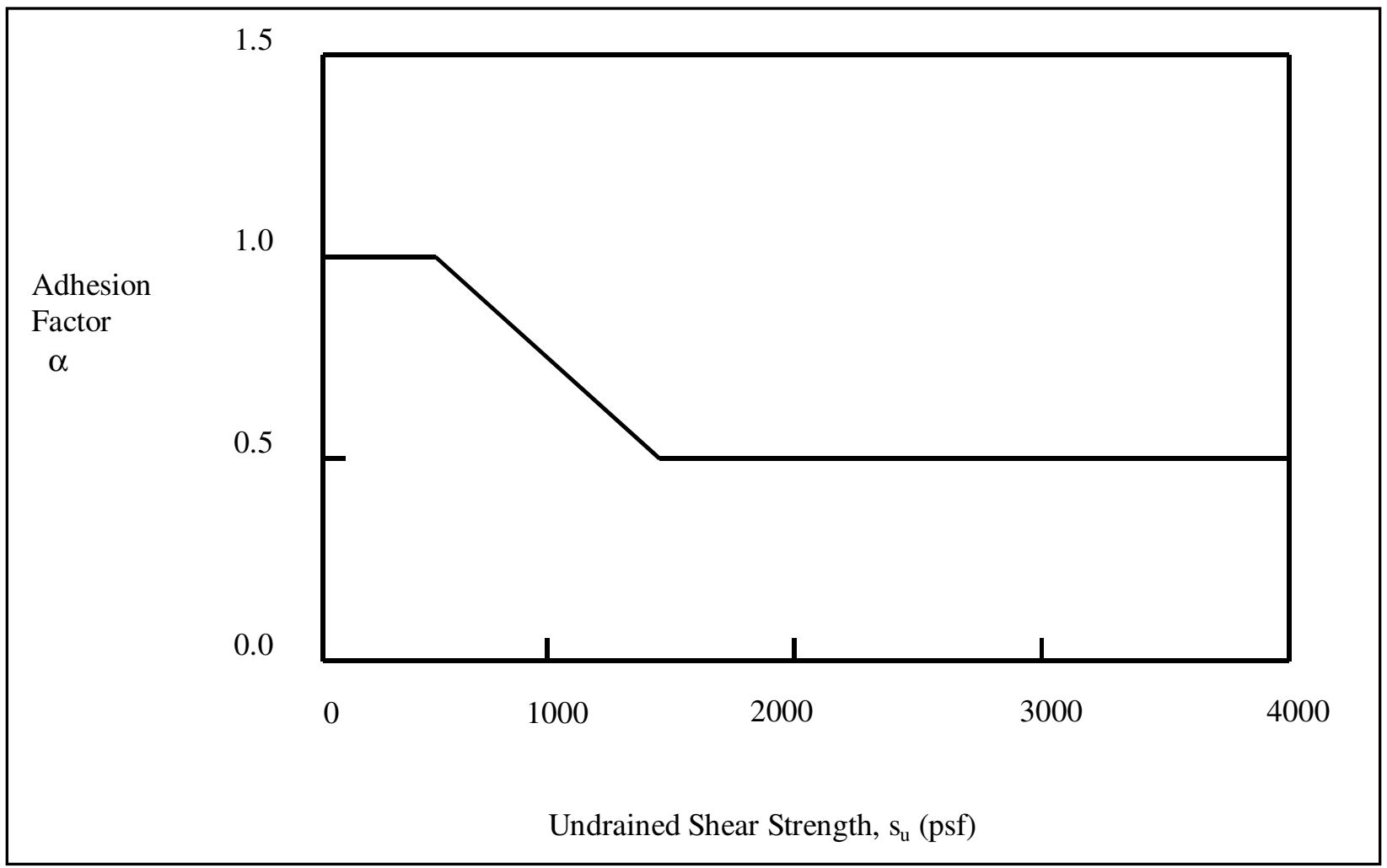

Figure 8.12. Adhesion factor versus undrained shear strength (after Figure 44, FHWA-RD-97-015)

Unit tip bearing capacity is given by the equation

$$
q=9 S_{u}
$$

Tip capacity is seldom relied upon for driven foundations in cohesive soils. To fully mobilize tip capacity in clay requires movement of 10 percent of the beam depth. Tip capacity is considered when computing the ultimate axial loadcarrying capacity of a soldier beam under certain conditions. When normal soldier beam settlement $(0.0015 \mathrm{H})$ can be tolerated and at least 50 percent of the axial load-carrying capacity results from skin friction, then tip capacity can be included in the ultimate axial capacity of the soldier beam. If soldier beam settlements must be kept to a minimum, then the ultimate capacity of driven soldier beams in clay should be computed using only skin friction. 
8.7.3.4 Drilled-in soldier beams in sand. Relationships for estimating the axial load-carrying capacity for drilled-in soldier beams in sands are based on equations developed by Reese and O'Neill (1988). The ultimate axial loadcarrying capacity of the drilled shaft is given by the equation

$$
Q_{u l t}=f_{s} A_{s}+q_{b} A_{t}
$$

where

$$
\begin{aligned}
A_{s} & =\text { surface area of the drilled shaft } \\
q_{b} & =\text { unit end bearing resistance } \\
A_{t} & =\text { cross-sectional area of the drilled shaft }
\end{aligned}
$$

The average unit skin friction, $f_{s}$, for the drilled shaft is

$$
f_{s}=\beta \sigma_{\text {vave }}^{\prime} \leq 4.0 \mathrm{ksf}
$$

where $\beta=1.5-0.135[(h+d) / 2]^{0.5}, 1.2 \geq \beta \geq 0.25$

and $\gamma^{\prime}$ is the effective unit weight of soil

Section 8.7.3.3.1 discusses the computation of the average effective vertical stress. Reese and O'Neill (1988) stated that $\beta$ in Equation 8.26 is independent of soil strength because drilling disturbance reduces the friction angle to a common value regardless of strength. The unit tip bearing, $q_{b}$, is given in Table 8.10

\subsubsection{Drilled-in soldier beams in} clay. Relationships for estimating the axial load-carrying capacity for drilled-

\begin{tabular}{||l||}
\hline \multicolumn{1}{||l||}{ Table 8.10 } \\
Recommended Values of \\
Unit Tip Bearing Capacity \\
for Drilled Shafts in Sand \\
(after Table 12, FHWA-RD- \\
97-130)
\end{tabular}
in-soldier beams in clay are based on equations developed by Reese and O'Neill (1988). The ultimate axial loadcarrying capacity of the drilled shaft is given by the equation

$$
Q_{u l t}=f_{s} A_{s}+q_{b} A_{t}
$$

The average unit skin resistance, $f_{s}$, is

$$
f_{s}=\alpha S_{u} \leq 5.5 \mathrm{ksf}
$$


where $\alpha$ is equal to 0.55 .

The unit tip bearing capacity, $q_{b}$, for clays is given by the equation

$$
q_{b}=N_{c} S_{u} \leq 80 \mathrm{ksf}
$$

where $N_{c}=6.0[1+0.2(d / b)] \leq 9$.

\subsubsection{Design issues for concrete backfill of predrilled soldier beam}

holes. General design recommendations for concrete backfill of predrilled holes include the use of structural concrete from the bottom of the hole to the excavation base and lean-mix concrete for the remainder of the hole. The design concept is to provide maximum strength and load transfer in the permanently embedded portion of the soldier beam while providing a weak concrete fill in the upper portion, which can easily be removed and shaped to allow lagging installation. However, contractors often propose to use lean-mix concrete for the full depth of the hole to avoid the delays associated with providing two types of concrete in relatively small quantities.

When using structural concrete with a minimum compressive strength of $21 \mathrm{MPa}(3,000 \mathrm{psi})$ and appropriate concrete placement procedures, the vertical load from the exposed portion of the wall is transferred from the steel soldier beam to the concrete, and the entire drilled shaft cross section is effective in resisting the vertical load. For this case, the wall can be analyzed as a drilled shaft using the methods presented in the previous sections. However, for lean-mix backfilled drilled shafts, the lean-mix concrete may not be sufficiently strong to allow vertical load transfer from the soldier beam to the concrete. The soldier beam may "punch" through the lean-mix concrete, in which case the drilled shaft cross section will not be effective in transferring load to the surrounding soil.

When designing the embedded portion of a permanent drilled-in soldier beam wall that is backfilled with lean-mix concrete, the following two analyses should be performed and the analysis that results in the greater embedment depth should be used.

- Analysis 1: Compute the required embedment depth assuming the drilledin soldier beam can be analyzed as a drilled shaft. Assume the full cross section is effective in resisting vertical load.

- Analysis 2: Compute the required embedment depth assuming that the soldier beam will punch through the lean mix concrete. The analysis procedures for driven soldier beams should be used. 


\section{Global Stability}

\subsection{Introduction}

Many of the design recommendations contained herein are taken from Sabatini, Pass, and Bachus (1999). The design of an anchored wall concentrates on achieving a final constructed wall that is safe against a range of potential failure conditions. These conditions are described in Sabatini, Pass, and Bachus (1999) and illustrated in Figure 9.1.

The stability analyses presented herein focus on whether the shear strength of the soil mass, and the location and magnitude of the resultant forces provided by the ground anchors and other structural components, are sufficient to provide an acceptable factor of safety. An adequate level of serviceability with respect to various external failure modes is also required.

\subsection{Stability of Anchored Wall Systems-Limit Equilibrium Methods}

\subsubsection{General}

Conventional limit equilibrium methods for slope stability can be used to evaluate the external stability of anchored wall systems. An anchored wall system is externally stable if potential slip surfaces passing behind or through the anchors have a factor of safety that exceeds the target factor of safety.

External stability analyses are particularly important in evaluating systems close to nearby structures or for situations in which soft soils exist below the wall.

For temporary support of excavation anchored systems constructed in soft to medium clay soils, external stability should be evaluated using short-term (i.e., undrained) strength parameters and temporary loading conditions. For permanent anchored wall systems constructed in soils, external stability for both short- and long-term conditions should be checked. For systems constructed in stiff clays, i.e., overconsolidated clays, external stability for short-term conditions may not be critical, but long-term conditions, using drained shear strength parameters, may be critical. External stability of wall supported by rock anchors is normally adequate; however, if the rock mass has planes of weakness that are orientated in a direction 


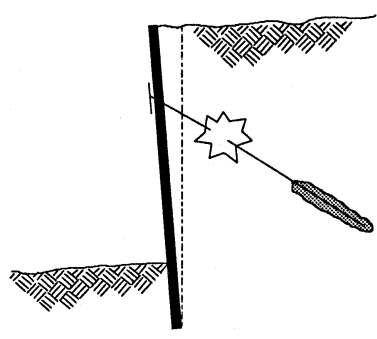

(a) Tensile failure of tendon

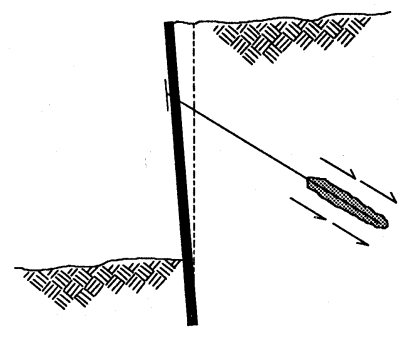

(b) Pullout failure of grout/ground bond

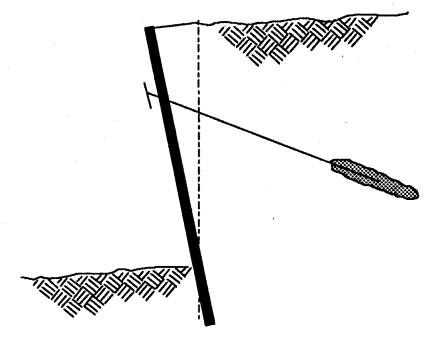

(c) Pullout failure of tendon/grout bond

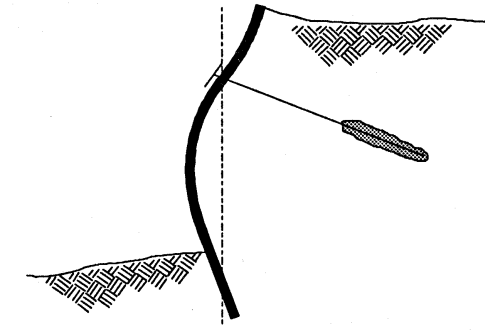

(d) Failure of wall in bending

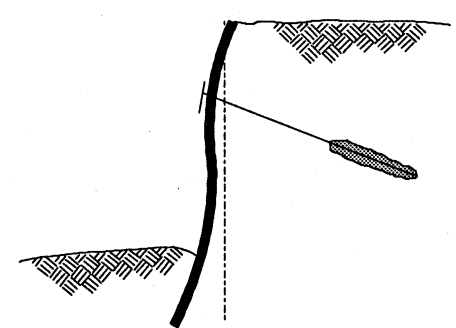

(e) Failure of wall due to insufficient passive capacity

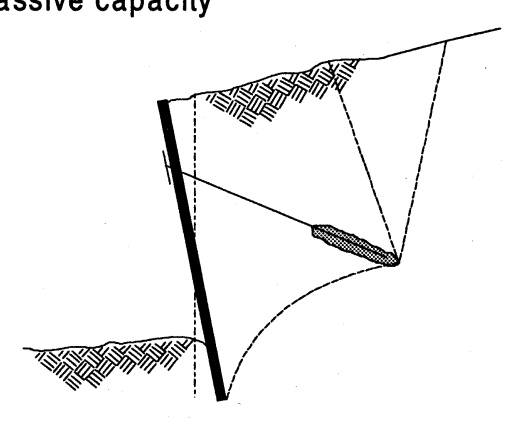

(g) Failure due to insufficient (f) Failure by forward rotation (cantilever before first anchor installed) axial capacity

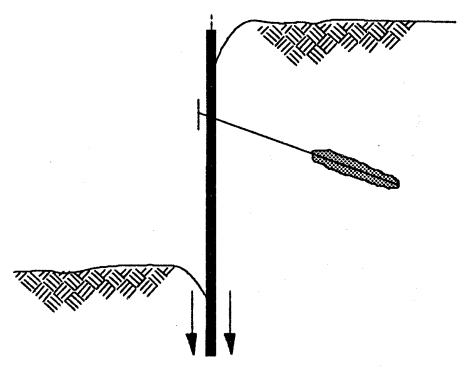

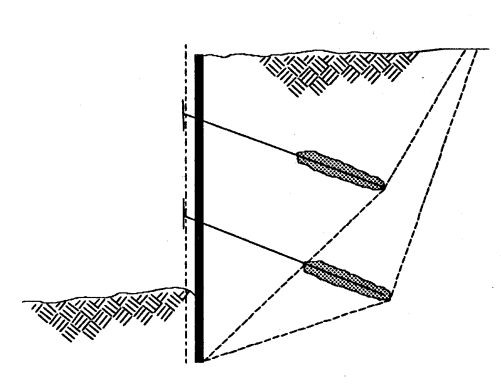

(i) Failure by sliding

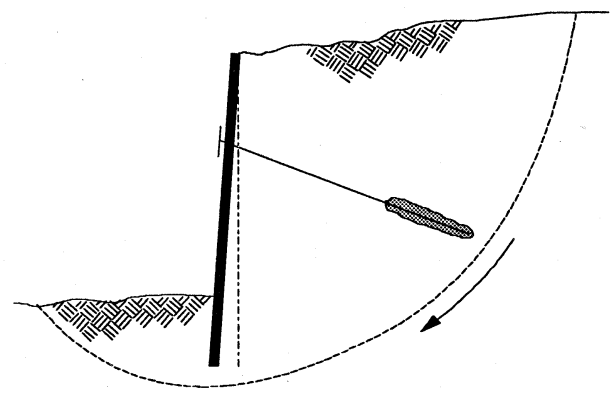

(j) Rotational failure of ground mass

Figure 9.1. Potential failure conditions to be considered in design of anchored walls (after Figure 11 of Sabatini, Pass, and Bachus 1999) 
that may affect stability, external stability should be checked for failure surfaces passing along those weak planes.

According to Sabatini, Pass, and Bachus (1999), the minimum acceptable factor of safety for external stability is 1.3. For permanent applications that are critical, a higher factor of safety (e.g., 1.5) may be used.

\subsubsection{Evaluation of stability using limit equilibrium}

To evaluate the stability of an anchored system, potential failure surfaces passing behind or through the anchors need to be checked. For walls with multiple levels of anchors, failure surfaces that pass behind each anchor should be checked (Figure 9.2). In checking a failure surface that passes behind a level of anchors, the failure surface may cross in front or through the anchor bond zone of other level(s) of anchors. In this case, the analysis may be amended to include a portion of the restraint force from the other anchor(s). If the failure surface passes in front of an anchor, the full design load can be modeled as a restraint force. If the failure surface crosses the anchor, a proportional magnitude of load, assuming that the anchor bond stress is distributed uniformly over the anchor bond length, can be modeled. Where stability requirements cannot be met, the anchors may be lengthened, or methods to improve anchor bond or load transfer mechanisms may be used.

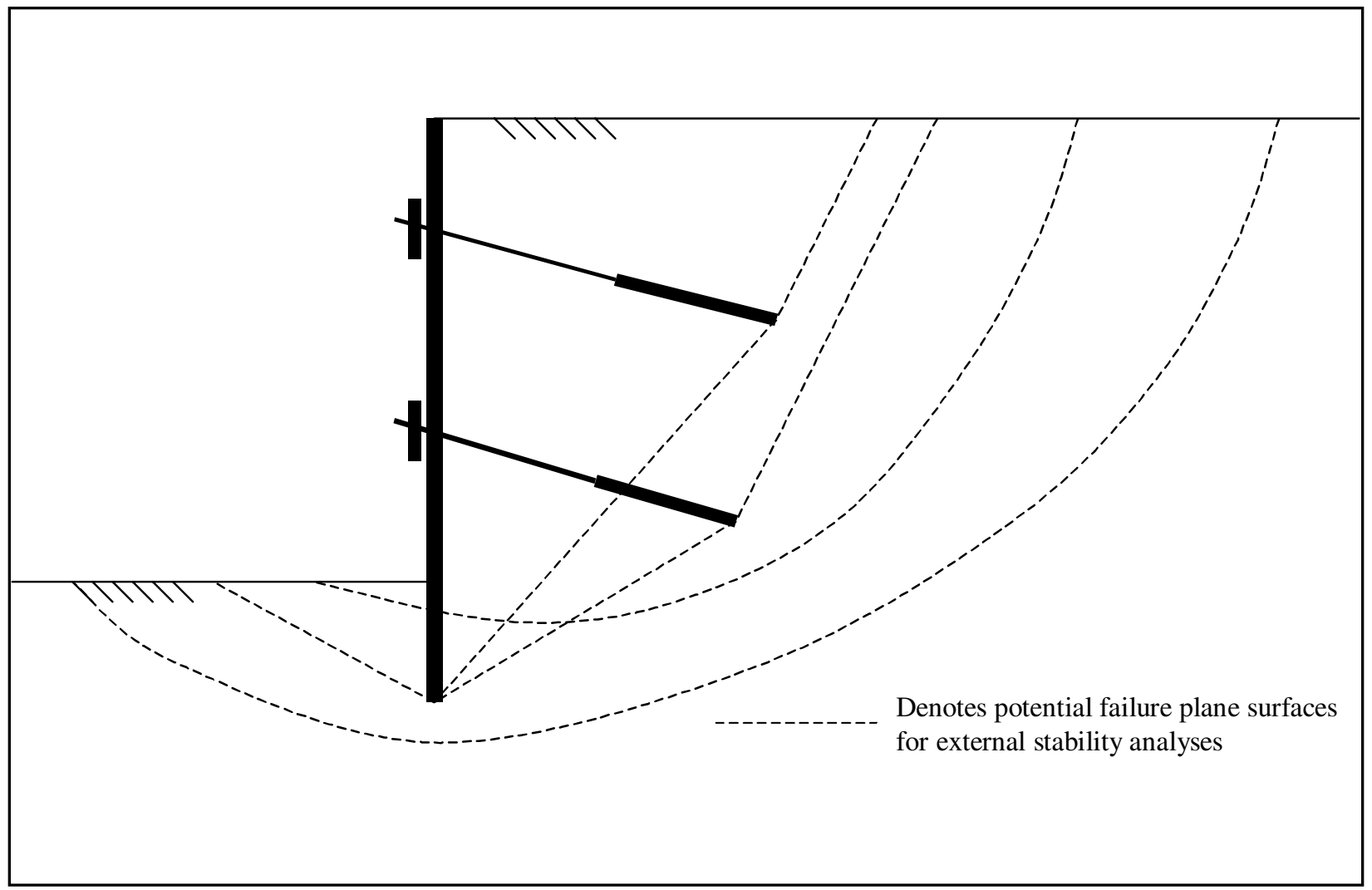

Figure 9.2. Failure surfaces for external stability evaluations (after Figure 52 of Sabatini, Pass, and Bachus 1999) 


\subsubsection{Limit equilibrium calculations}

9.2.3.1 Overall approach. Slope stability computer programs that incorporate the equilibrium method of slices can be used to examine the stability of anchored wall systems. However, the current state of the practice does not include a generally accepted method of modeling the restraint force provided by the prestressing ground anchors (Sabatini, Pass, and Bachus 1999). Methods used in practice distribute the anchor forces to slices in different ways, and each slope stability program includes one or several of these methods. For this reason, caution should be exercised when using limit equilibrium methods to calculate required forces to restrain a slope. Calculated forces should be reviewed critically and compared to solutions based on simpler hand-calculation methods. A detailed discussion on the use of limit equilibrium methods for the analysis of anchored systems is provided in Long, Weatherby, and Cording (1998).

Two methods that can be used to model ground anchor restraint forces are introduced below.

- Method 1, Apply surcharge or concentrated force to wall: If a surcharge or concentrated force equivalent to the total ground anchor restraint force is applied at the wall, a very large vertical force component will be transmitted to the slice base on which it acts. The calculated factor of safety for this slice will be unrealistically high. This method seems realistic in that the large compressive forces imposed by ground anchors are applied to the face. However, the large increase in vertical force to only one slice while nearby slices remain unaffected seems incorrect since the ground anchors presumably increase the normal forces on the critical surface in a more widespread fashion.

- Method 2, Apply concentrated force to slice base where failure surface crosses anchor: With this method, the normal stress on the slice where the failure surface and the anchor intersect is increased while nearby slices remain unaffected. This method is commonly used for modeling geosynthetic reinforcement. This method suffers from the same limitations as Method 1 in that the increase in normal force on the failure surface is highly localized. (This may also depend upon the assumptions regarding interslice forces made by the particular slope stability software being used in the analysis.)

For both of these methods the increase in normal stress on the critical potential failure surface is highly localized and not likely to be consistent with the actual distribution of stresses imposed by the ground anchors. For a case where the failure plane is at a constant inclination and the soil strength along the failure plane is homogeneous, both of these methods provide similar results. For failure surfaces that are irregular and for highly stratified soils, it is likely that these two methods will result in different calculated factors of safety.

A reasonable approach to using limit equilibrium methods for evaluating the internal or external stability of anchored wall systems is to perform an analysis using either Method 1 or Method 2. The anchored wall is internally stable if the 
anchor bond length(s) is (are) located behind the critical surface that corresponds to a factor of safety of one. The anchored wall is externally stable if the factor of safety for the failure surfaces that pass behind the back of the ground anchors is equal to or greater than the design target value (typically 1.3). Ideally, analysis using both methods should be performed, and the results from each compared to the design target factor of safety. If available, a slope stability computer program that has the capability to search for critical failure surfaces using a slice equilibrium method that satisfies both moment and force equilibrium should be used. If this feature is not available, the search for the critical failure surface may be performed using simpler force or moment equilibrium methods. Some programs may not offer the capability to use a method that satisfies both force and moment equilibrium to perform a general search for a critical potential failure surface. However, they most likely offer the capability to use a method that satisfies both force and moment equilibrium to calculate the factor of safety for a specific failure surface.

If the calculated factors of safety from one or both analyses exceed the target value, then the calculated anchor restraint force can be used for design. If the calculated factors of safety are less than the target value, the anchor force can be increased until the target value is reached. The user should evaluate whether the calculated restraint force required to meet the target factor of safety is reasonable. If the calculated restraint force seems excessively large or small, or if changes in analysis parameters (e.g., inclination of failure surface) result in very large variations in calculated factors of safety, then additional analyses should be performed.

Both of these methods for evaluating the total stabilizing load for an anchored wall are described herein. The analyses should be performed for each critical design cross section. Noncircular (i.e., planar) failure surfaces should be used where the soils are predominantly cohesionless or where the failure surface is located along a well-defined interface. For analysis of temporary walls constructed in weak (i.e., soft to medium) cohesive soils, a circular failure surface should be used. Table 9.1 provides a general outline for performing the analysis.

\begin{tabular}{|}
$\mid$\begin{tabular}{|l||}
\hline Table 9.1 \\
Procedure to Evaluate Total Earth Load Using Slope Stability \\
Computer Programs (after Sabatini, Pass, and Bachus 1999, \\
Table 16)
\end{tabular} \\
\hline \hline Step1 & $\begin{array}{l}\text { Develop cross-section geometry including subsurface stratigraphy, external surcharge } \\
\text { loading, and water pressures. }\end{array}$ \\
\hline Step 2 & Assign shear strength and unit weight to each soil or rock layer. \\
\hline Step 3 & $\begin{array}{l}\text { Select limit equilibrium method that satisfies both force and moment equilibrium and } \\
\text { appropriate critical surface search parameters. }\end{array}$ \\
\hline Step 4 & $\begin{array}{l}\text { Apply surcharge or concentrated forces to wall (Method 1) or model the ground anchors } \\
\text { as reinforcements (Method 2). Model the wall face with a slight batter to avoid anomalous } \\
\text { numerical instabilities. }\end{array}$ \\
\hline Step 5 & Evaluate critical surface and factor of safety for the load applied in Step 4. \\
\hline Step 6 & $\begin{array}{l}\text { Repeat Steps } 4 \text { and 5, increasing the surcharge or concentrated forces (Method 1), or } \\
\text { reinforcement tension (Method 2), until the target factor of safety is obtained. }\end{array}$ \\
\hline
\end{tabular}


9.2.3.2 Method 1 analysis. In using Method 1, the following cases are considered: Case 1, the wall penetrates the potential critical failure surface, and Case 2, the wall does not penetrate the critical failure surface. These cases are illustrated in Figure 9.3.

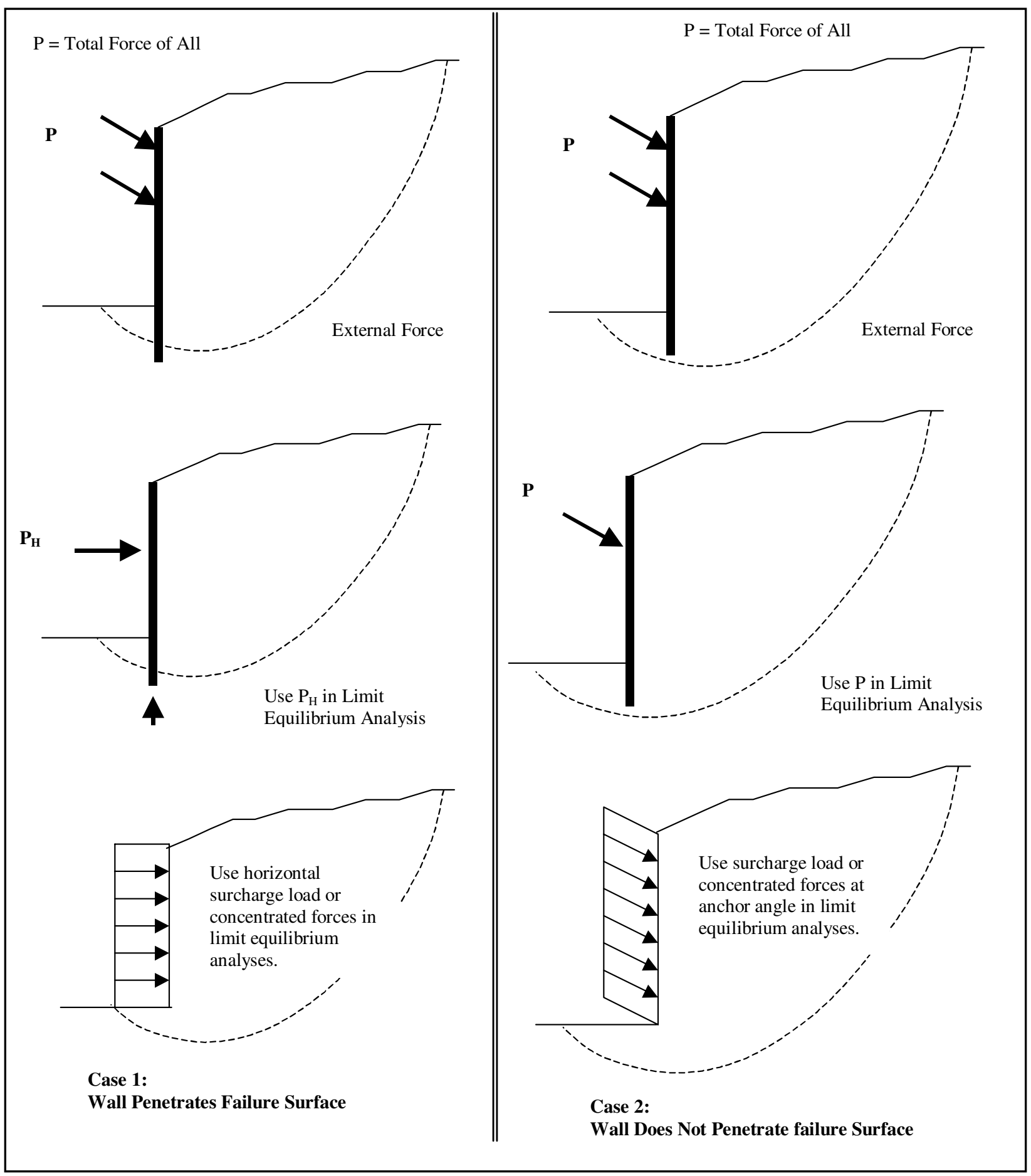

Figure 9.3. Modeling the ground anchor force in limit equilibrium analysis (after Weatherby 1998) 
For Case 1, it is assumed that the vertical component of the anchor load is transmitted below the critical failure surface at the wall location. Thus, only the horizontal component of the ground anchor force is transmitted to the failure surface. The total surcharge load must be resisted by the ground anchors and the lateral capacity of the portion of the wall that extends below the failure surface. For cases where homogeneous weak cohesive soil extends far below the base of the excavation (at least approximately 20 percent of the wall height), the potential critical failure surface may likely penetrate significantly below the bottom of the excavation. For these cases, relatively large loads will need to be resisted by the lower anchors. When modeling the ground anchor restraint force using Method 1 and the procedure outlined in Table 9.1, the resultant of the surcharge or concentrated anchor forces used to model the total ground anchor restraint force should be located between 0.3 and $0.5 H$ measured from the bottom of the excavation. A procedure for evaluating the total load required to stabilize a cut, for which the failure surface penetrates significantly below the wall, is described in Table 9.2 and is illustrated in Figure 9.4. With this procedure, the location of the resultant of the total load required to stabilize the system to the target factor of safety will get progressively lower in the wall as the failure surface penetrates deeper.

\begin{tabular}{|l}
\hline \hline Table 9.2 \\
Procedure to Evaluate Total Lateral Earth Load for Anchored \\
Systems Constructed in Weak Cohesive Soils (after Sabatini, Pass, \\
and Bachus 1999, Table 17)
\end{tabular}

9.2.3.3 Method 2 analysis. With this method, the ground anchor is considered to be a high-capacity reinforcement. The axial anchor force is modeled along the length of the anchor and the anchor bond zone. The axial force in the reinforcement is assumed to vary linearly from the full capacity for all positions in front of the anchor bond zone, to zero force for the end of the ground anchor. This concept is similar to the stability analysis involving soil nails as described in Byrne, Cotton, Porterfield, Wolschlag, and Ueblacker (1998). Multiple levels of anchors may be modeled, so the user should assume a reasonable layout of anchors and anchor inclinations in performing the analysis. If the failure surface crosses the wall, the additional restraint provided by the wall may be modeled. 


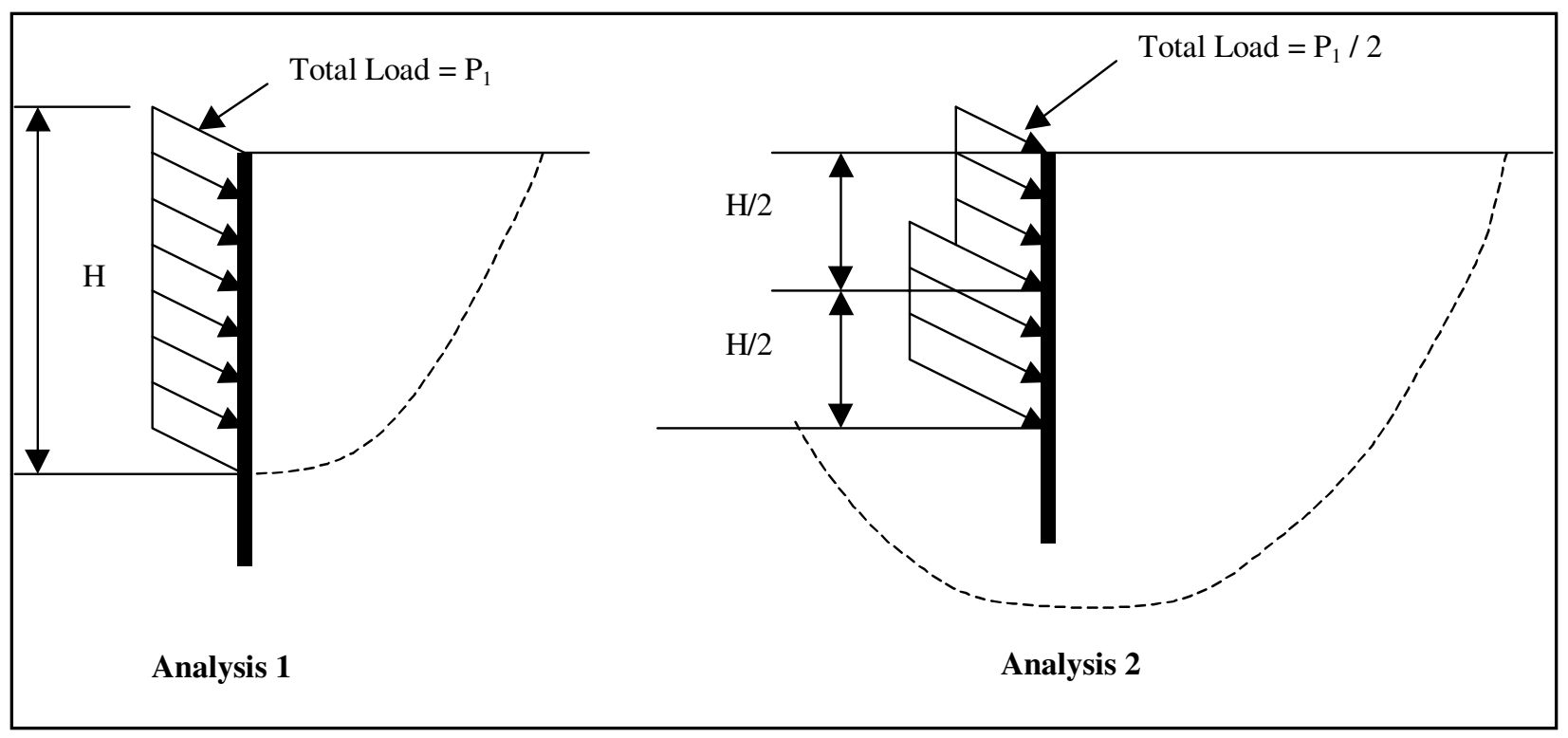

Figure 9.4. Limit equilibrium analyses used to evaluate total lateral earth load for anchored systems constructed in weak cohesive soils (after Weatherby 1998)

\subsubsection{Modeling lateral wall resistance in limit equilibrium analysis}

When the critical potential failure surface intersects the embedded portion of the wall, the additional resistance provided by the wall may be included in a limit equilibrium analysis. The resisting force to be used in the limit equilibrium analysis is the lesser of the following: the shear capacity of the wall, or the total passive force that may be developed in the soil over the length of the wall from the failure surface to the bottom of the wall. The shear capacity of the wall is a constant and is assumed to be equal to the allowable shear capacity of the vertical wall element.

\subsubsection{Comparison of methods to evaluate required earth loads in homogeneous soils}

9.2.5.1 General. This section provides comparisons between apparent earth pressure and limit equilibrium based on calculations for evaluating required restraint (anchor) forces in relatively homogeneous soils. These comparisons (Sabatini, Pass, and Bachus 1999) were performed for vertical walls with either planar or circular failure surfaces and where the soil strength properties $\left(\phi^{\prime}\right.$ or $\left.S_{u}\right)$ were constant for the entire profile analyzed.

9.2.5.2 Cohesionless soils. Three methods - the apparent earth pressure envelope method, the sliding wedge method, and the limit equilibrium methodare available for determining the total earth load $\left(P_{R E Q}\right)$ to stabilize a cut in cohesive soils. For design of the wall, the ground anchors and the reaction force at the excavation subgrade carry this total load. In Table 9.3, the normalized total earth load, 


$$
K_{R E Q}=\frac{P_{R E Q}}{\frac{1}{2} \gamma H^{2}}
$$

required to stabilize a cut in cohesionless soil is compared for the three aforementioned methods. The apparent earth pressure envelope produces a total earth load equal to

$$
0.65 K_{A} \gamma H^{2}
$$

which is 1.3 times greater than for active Rankine conditions. For the sliding wedge and limit equilibrium analysis, a factor of safety of 1.3 on the shear strength was used. For the limit equilibrium analysis, a uniform horizontal surcharge was applied to the wall face and increased until the target factor of safety was achieved (i.e., Method 1).

The results indicate that all three methods give similar results. When designing anchored walls in reasonably homogeneous cohesionless soils for which competent soils exist below the wall excavation, any of these methods will provide reasonable results, but using the apparent earth pressure envelope to calculate required anchor loads is the most expedient.

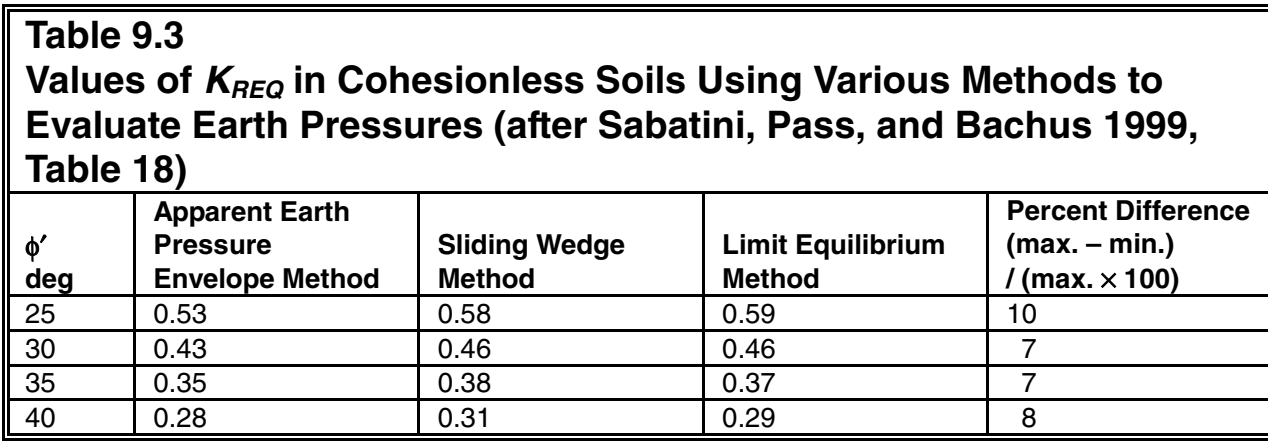

9.2.5.2 Cohesive soils. Limit equilibrium methods were also assessed for evaluating the total earth load for anchored systems in purely cohesive soils. For temporary anchored systems in soft to medium clays with $N_{S}>4$, computed earth loads were compared using Henkel's method, Rankine method, and limit equilibrium solutions. These results are shown in Figure 9.5. The stability number, $N_{s}$, is defined as $\frac{\gamma H}{S_{u}}$.

Limit equilibrium methods include Bishop's method, Spencer's method, and the Corps of Engineers' method. Of these limit equilibrium methods, Spencer's method is the only one that satisfies both moment and force equilibrium. Results indicate that limit equilibrium methods compare favorably to Rankine analyses where the failure surface intersects the corner of the wall. When the failure surface extends below the excavation (e.g., $d / H=0.2$ in Figure 9.5), Henkel's 


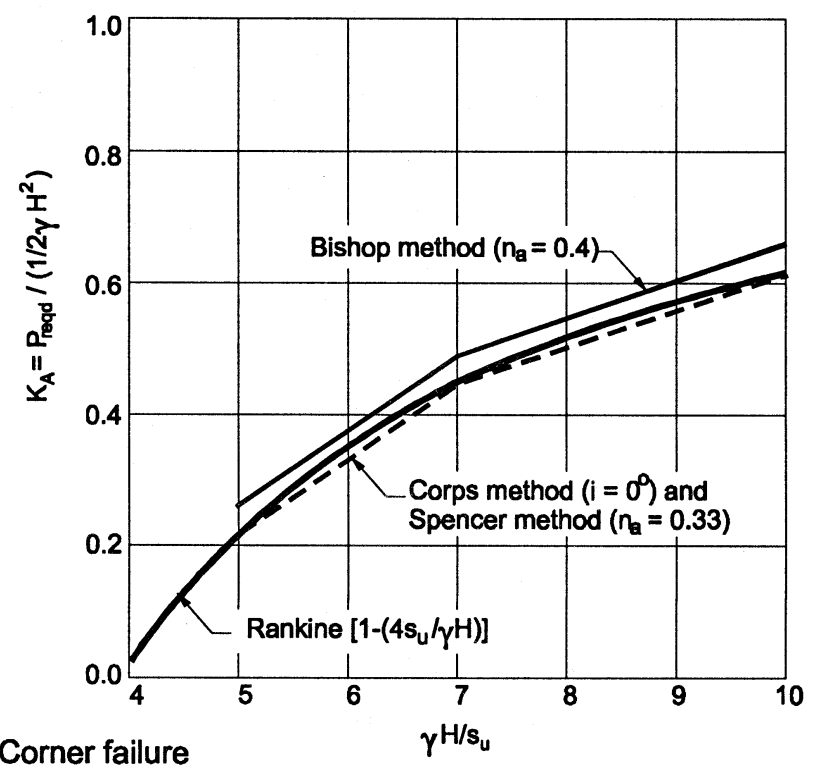

(a) Corner failure

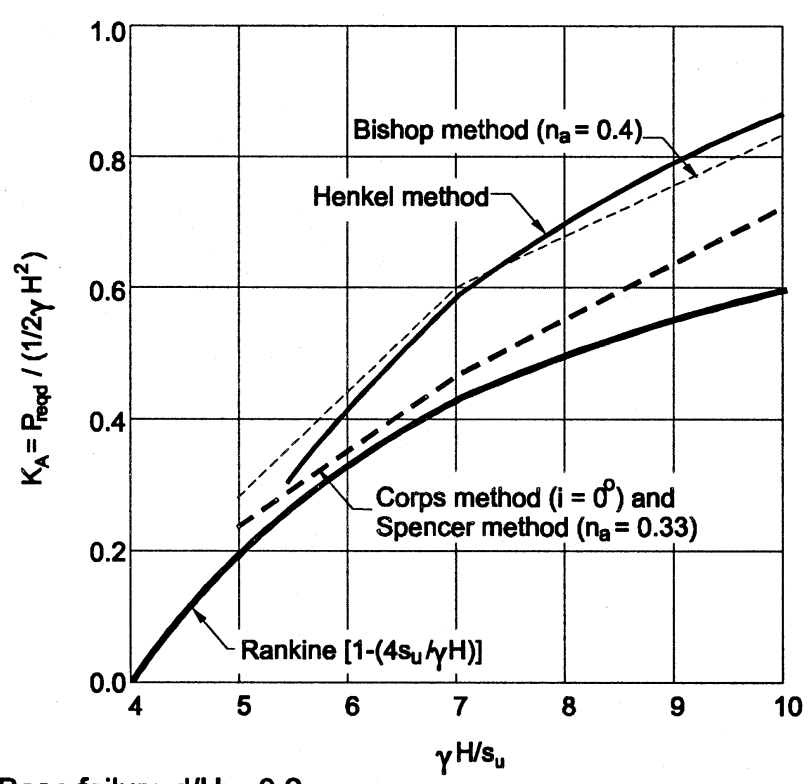

(b) Base failure $\mathrm{d} / \mathrm{H}=0.2$

Figure 9.5. Comparison of limit equilibrium methods for cohesive soils (after Figure 50 of Sabatini, Pass, and Bachus 1999)

method and Bishop's method are in reasonable agreement and are upper bounds. For cases in which the critical potential failure surface extends below the base of the excavation and where $N_{S}>5$, the Rankine analysis results are unconservative. For those cases, either Henkel's method or limit equilibrium analysis methods should be used to evaluate the total earth load. The total load should then be redistributed into an apparent pressure diagram using the Terzaghi and Peck diagram for soft to medium clays. 


\subsection{Base Stability and Heave in Cohesive Soils}

The earth pressure diagrams described in previous chapters apply only when the soil at the bottom of the wall is not near a state of plastic equilibrium (failure). Excavations in deep deposits of soft to medium clay have moved excessively because the weight of the retained soil exceeds the bearing capacity of the soil at subgrade or a deep-seated failure develops. Soldier beam walls with timber lagging constructed in granular soils are not subject to basal instability since the walls are free draining. Special attention must be given to assessing the base stability of cuts in soft to medium clays and the effect of base stability on apparent earth pressures. The potential for base heaving failures in cuts in soft to medium clays can be evaluated using procedures described in EM 1110-1-1905. Substantial increases in apparent earth pressures have resulted when the weight of the retained soil exceeds or approaches the bearing capacity at the base of the excavation.

Continuous tieback wall systems constructed in granular soils may be subject to unbalanced seepage forces when the groundwater level behind the wall is higher than the groundwater level inside the wall. If the wall does not penetrate below the bottom of the excavation sufficiently, the upward flow of water inside the wall may create base instability. The Navy's Design Manual 7.2 (NAVFAC 1982) presents procedures for determining the toe penetration required to prevent base instability resulting from unbalanced seepage pressures. 


\section{Serviceability of Tieback Wall Systems}

\subsection{General}

Issues related to tieback wall serviceability are discussed in this chapter. These issues include displacement control, drainage and seepage control, and corrosion protection. Much of the information contained herein is taken from Sabatini, Pass, and Bachus (1999).

\subsection{Displacement Control}

\subsubsection{General}

Depending on project constraints, requirements with respect to control of wall and ground movements will vary. Permanent anchored walls constructed in granular soils with no nearby structures, utilities, or railways pose little concern with respect to movements. Wall and ground movements, however, may be the primary design issue for tieback walls constructed in urban areas, or in areas adjacent to structures, utilities, and railways. Estimates of wall and ground movements are typically made using semi-empirical relationships developed from past performance data.

\subsubsection{Estimating wall movements and ground settlements}

Based on limited observations, the maximum lateral wall movements for welldesigned anchored walls with high-quality construction in sands and stiff clays average approximately $0.002 \mathrm{H}$, with a maximum of approximately $0.005 \mathrm{H}$, where $H$ is the height of the wall. Maximum vertical settlements behind a wall constructed in these materials average approximately $0.0015 \mathrm{H}$, with a maximum of approximately $0.005 \mathrm{H}$. To evaluate the settlement profile behind an anchored wall, the curves shown in Figure 10.1 can be used. Curves I and II are commonly used for permanent anchored walls. Because of the variables involved, designers are cautioned to use Figure 10.1 only as an index of the possible magnitude of ground settlements to be expected adjacent to tieback walls. Settlements increase rapidly for walls constructed in soft to medium clays where basal stability is marginal. 


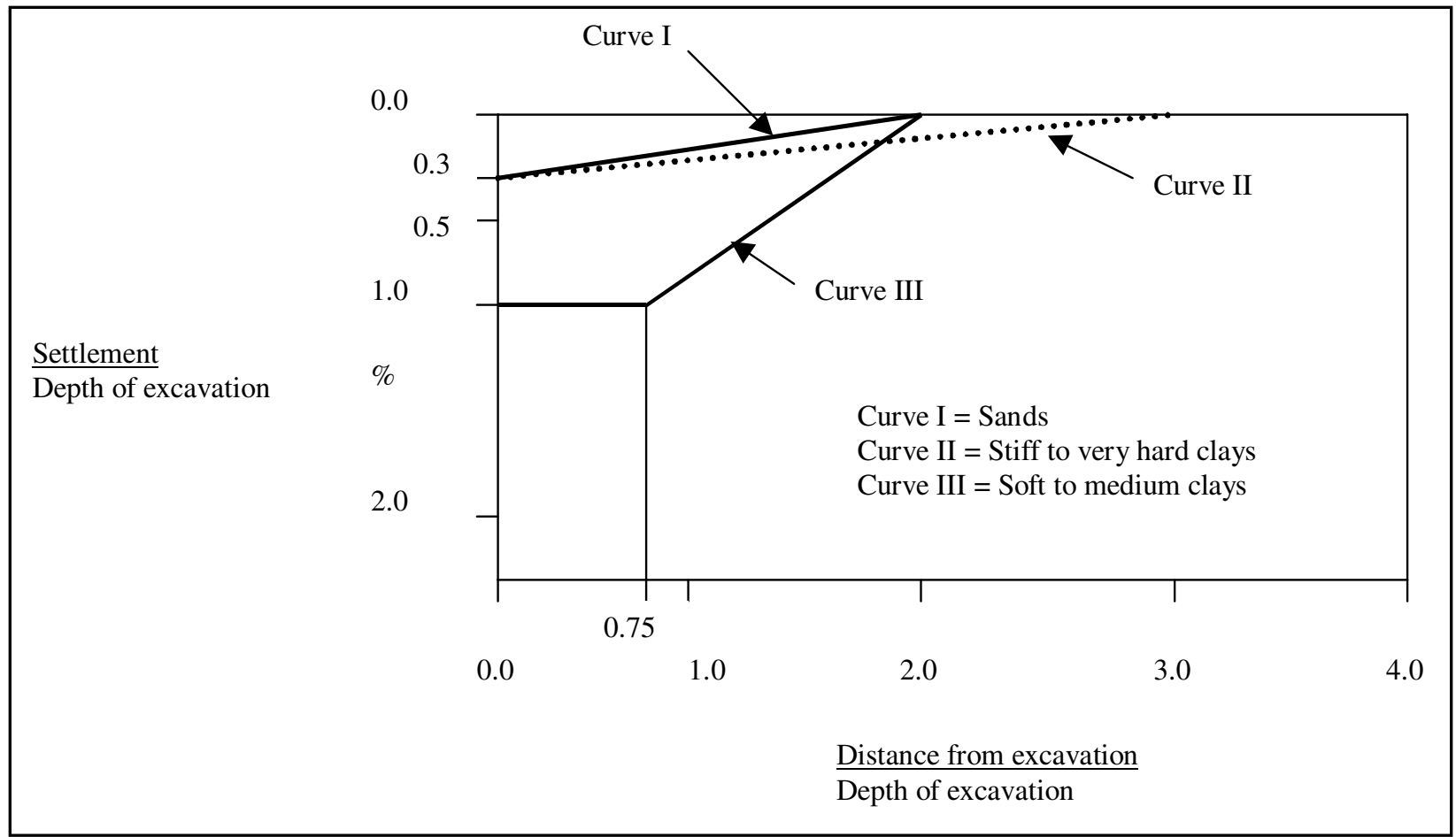

Figure 10.1. Settlement profile behind anchored walls (after Figure 59 of Sabatini, Pass, and Bachus, 1999)

Several types of movement are associated with flexible anchored walls. These include (1) cantilever movements associated with installation of the first anchor, (2) wall settlement associated with mobilization of end bearing, (3) elastic elongation of the anchor tendon associated with a load increase, (4) anchor yielding or load redistribution in the anchor zone, and (5) mass movements behind the ground anchors. The last three components of deformation result in translation of the wall and are relatively small for anchored walls constructed in competent soils. Excessive vertical settlements of the wall may induce significant lateral wall movements in addition to causing high stresses at the wall/anchor interface. Wall settlements can be minimized by installing ground anchors at flat angles and by designing the embedded portion of the wall to carry applied axial loads.

\subsection{Drainage and Seepage Control}

For anchored wall systems with cast-in-place concrete wall facing, collection of subsurface flow is usually achieved with prefabricated drainage elements placed between the wall and the lagging. Full-length elements are usually attached to the timber lagging after the design final excavation grade is reached. Single strips can be placed at design horizontal spacings along the wall. Where shotcrete is used in lieu of timber lagging, special considerations are required to ensure drainage behind the shotcrete. Typically, prefabricated vertical drains are installed in segments against the soil face with spikes. The segments are spliced by shingling the next segment over the previously placed length after each lift is complete. An overlap length of one strip width is adequate. 
Where precast facings are used, the space between the temporary wall face and the permanent facing may be backfilled with gravel. The gravel backfill acts as a drainage element. Water intercepted in the drainage element flows downward to the base of the wall where it is removed by collector pipes or conveyed through permanent facing in longitudinal outlet pipes or weep holes.

In applications where subsurface flows are large, horizontal drains may be used to remove water from behind the wall. A horizontal drain is a small-diameter perforated pipe that is advanced into a nearly horizontal drill hole in an existing slope. For example, an anchored wall constructed on or at the base of a steep slope will likely interfere with pre-existing natural drainage paths. This interference may cause hydrostatic pressures resulting from trapped water to build up against the wall. To relieve these pressures, horizontal drains can be installed at appropriate vertical and horizontal spacing along the wall alignment. Horizontal drains extend back from the wall face a sufficient distance to intercept subsurface flow beyond the critical potential failure surface. Several factors related to the construction of horizontal drains have limited their use for anchored system applications. These factors are described below.

- Horizontal drains should not be installed until after the final excavation grade is reached, unless a perched water table exists above the final excavated grade. This higher drain installation may result in water flowing into the excavation during construction.

- The alignment of the drains must be carefully controlled to avoid interference with the ground anchors. Splaying of multiple drains from a single entry point is not recommended.

- Horizontal drains usually cannot achieve lowering of the water table to a finished grade as the lowest elevation at the wall is controlled by construction equipment height and the drains are sloped upward.

- Special designs are required to collect the effluent from the drains to preserve the aesthetics of the wall face.

Surface drainage for anchored walls is usually achieved by directing water away from the wall face either by grading or by collecting and transporting surface water in ditches or pipes. To minimize surface water that can enter the excavation during construction and weaken the soils inside the excavation, dikes can be constructed on the ground surface near the top of the wall, or the vertical wall element can extend above the ground surface.

\subsection{Corrosion Considerations in Design}

\subsubsection{Introduction}

Protecting the metallic components of the tendon against the detrimental effects of corrosion is necessary to ensure adequate long-term durability of the ground anchor. Corrosion protection for ground anchor tendons includes one or more physical barrier layers that protect the tendon from the corrosive environment. The barrier layer includes anchorage covers, corrosion-inhibiting compounds, sheaths, encapsulations, epoxy coatings, and grouts. The selection of the physical barrier depends on the design life of the structure (i.e., temporary or 
permanent), aggressivity of the ground environment, the consequences of failure of the anchored system, and the additional cost of providing a higher level of protection.

\subsubsection{Corrosion and effects on ground anchors}

10.4.2.1 Mechanisms of metallic corrosion. Corrosion is an electrochemical reaction involving a base metal, oxygen, and water in which the metal returns to its natural oxidized state. In the context of a ground anchor, corrosion is most common on steel tendons that are improperly stored at a construction site. Less common are reactions that occur with galvanic corrosion in which, for an electrolytic ground environment, metal is lost with the flow of current from one location on the prestressing steel to another location, or to a nearby metal object. These reactions may occur between (1) nearby locations on the surface of the prestressing steel, (2) locations on the prestressing steel and a nearby metal object, or (3) locations on the prestressing steel in aerated soils (e.g., soils above the groundwater table and in fill and sands) and in nonaerated soils (e.g., soils below the groundwater table and in clays). Corrosion can occur when significant variations exist in the ground along the ground anchor length, particularly with $\mathrm{pH}$ and resistivity. The potential for excessive loss of metal by corrosion in soil is high in the following environments: (1) soil near the groundwater table, (2) soil exhibiting low $\mathrm{pH}$, (3) soils with high concentrations of aggressive ions such as chlorides or sulfides, and (4) sites where stray currents are present.

10.4.2.2 Types of corrosion for prestressing steel. Corrosion of prestressing steel may be classified according to the following six major types: general corrosion, localized corrosion, stress corrosion/hydrogen embrittlement, fatigue corrosion, stray current corrosion, and bacterial attack. Corrosion of unprotected prestressing steel usually initiates during storage with general corrosion. General corrosion causes an insignificant amount of metal loss; however, it may lead to either localized corrosion or stress corrosion/hydrogen embrittlement, which is a major cause of documented ground anchor failures. The last three types of corrosion need only be considered under special loading or ground conditions.

General corrosion occurs as a thin layer of rust uniformly distributed on the bare surface of unprotected prestressing steel. This type of corrosion is often observed on bare prestressing steel left exposed to weather during onsite storage. Where exposure times are limited, or adequate protection is provided, general corrosion usually involves only negligible loss of metal. In general, a light surface coating of rust is not considered detrimental to the tendons. The inspector can easily determine if the surface rust can be removed by wiping the rust from a short section and examining the exposed steel area for pits and cracks. Lightly rusted tendons can be inserted into the drill hole without rust removal.

Localized corrosion appears as pitting or crevices at one or more locations on the unprotected prestressing steel. In very aggressive ground conditions, unprotected prestressing steel may become severely pitted after only a few weeks of exposure. Complete encapsulation of the tendon is required in aggressive soils to prevent localized corrosion. 
Stress corrosion/hydrogen embrittlement occurs as cracks in steel at pit locations and is of a particular concern for high-strength steels used to manufacture prestressing elements. As stress corrosion progresses, tensile stresses present in the steel become highly concentrated. This stress concentration may cause a crack to develop, which may advance into the uncorroded metal at the bottom of the pit. With time, cracks may propagate into the metal to a sufficient depth to result in rupturing of the prestressing element. Pits or cracks on the tendon surface are adequate reason for rejection of the tendon.

Fatigue corrosion develops under cyclic loading as a progression of corrosion from its initiation to a cracking of a prestressing element. This type of corrosion is relatively uncommon in prestressing steel, as most ground anchors are not subject to severe cyclic loading.

Stray current corrosion occurs as pitting of prestressing steel when subject to prolonged exposure to stray electrical currents. Stray currents in the ground result from the discharge of direct electrical current from power sources such as electric rail systems, electrical transmission systems, and welding operations and is particularly damaging in the marine environment. Power sources beyond a distance of 30 to $60 \mathrm{~m}$ (100 to $200 \mathrm{ft}$ ) from a ground anchor are not believed to cause a significant amount of stray current corrosion. Protection of anchors from stray currents commonly involves complete electrical isolation of the prestressing steel from the ground environment with a nonconducting barrier such as plastic.

Bacterial attack occurs as pitting of unprotected prestressing steel. The potential for bacterial attack should be considered in marshy ground and sulfatebearing clay soils located below the groundwater table. Such ground conditions are considered aggressive and, therefore, encapsulated tendons should be used in these types of ground. Field and laboratory tests are available to test for the presence of sulfates and sulfides.

\subsubsection{Corrosion protection of ground anchors}

10.4.3.1 Requirements of corrosion protection systems. Corrosion protection systems protect the ground anchor from corrosion by providing one or more impervious physical barrier layers around the tendon. Corrosion protection systems should satisfy the following criteria:

- Ensure that the service life of the anchor with respect to corrosion failure is at least equal to the anticipated service life of the anchored wall system.

- Produce no adverse impacts on the environment or reduce the capacity of the anchor.

- Allow unrestricted movement of the tendon along the unbonded length such that all load is transferred to the bond length.

- Comprise materials that are chemically stable and nonreactive with adjacent materials.

- Require no maintenance or replacement (with few exceptions) during the service life of the anchor. 
- Be sufficiently strong and flexible to withstand deformations that occur during stressing of the tendon.

- Be durable to withstand handling without damage during manufacture, transport, storage, and installation.

\subsubsection{Design of corrosion protection systems.}

10.4.3.2.1 General. The design objective of a corrosion protection system is to protect the steel components of the ground anchor. The components of a corrosion protection system combine to provide an unbroken barrier for each part of the tendon - the anchorage, the unbonded length, and the bond length—and for the transitions between them. Steel components of the anchor include the anchor head, bearing plate, trumpet, prestressing steel, and couplers (where used).

Components of the corrosion protection system include the following: (for the anchorage) a cover or concrete embedment, a trumpet, and corrosion inhibiting compounds or grout; (for the unbonded length) grout and a sheath filled with corrosion-inhibiting compounds or grout; and (for the bond length) grout and encapsulations with centralizers or epoxy coatings. These components are shown for bar and strand tendons in Figures 10-2 through 10-4 and are briefly described below. Requirements for installation of the components of the corrosion protection system are also provided in this section.

- Anchorage covers: Anchorage covers protect the anchor head and exposed prestressing steel from corrosion and physical damage, and are fabricated from steel or plastic.

- Trumpet: The trumpet protects the back of the bearing plate and prestressing steel in the transition from the anchorage to the unbonded length and is fabricated from steel or polyvinyl chloride pipe.

- Corrosion-inhibiting compounds: These compounds protect steel components of the anchorage and unbonded length, are nonhardening, and include greases and waxes.

- Grout: Grout protects the prestressing steel in the unbonded and bond lengths and may be either cement-based or polyester resin. Polyester resin grout is not generally considered to provide a corrosion protection layer, as gaps in the resin coverage will leave the prestressing steel unprotected. Grouts are also used to fill sheaths, encapsulations, covers, and trumpets. 


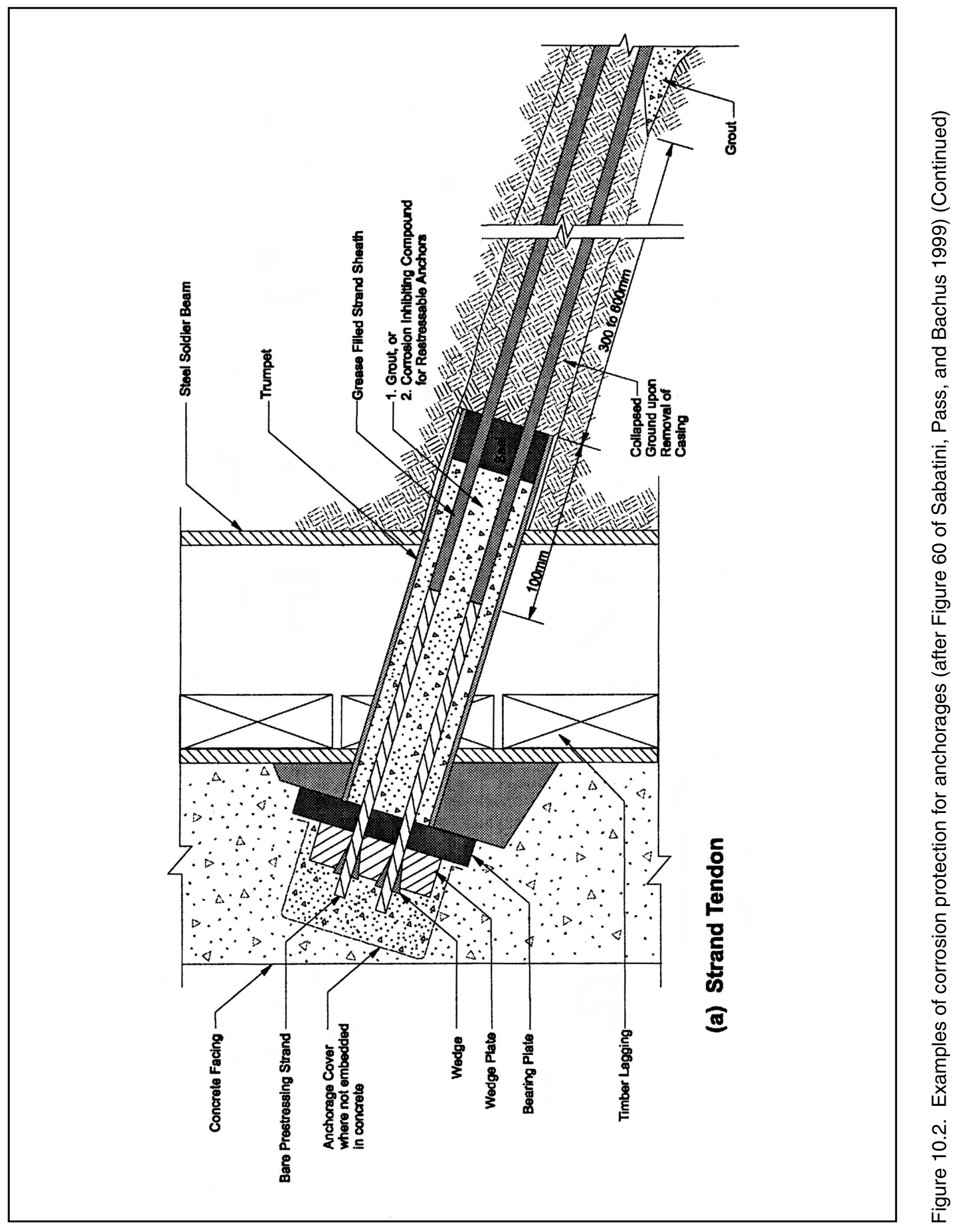




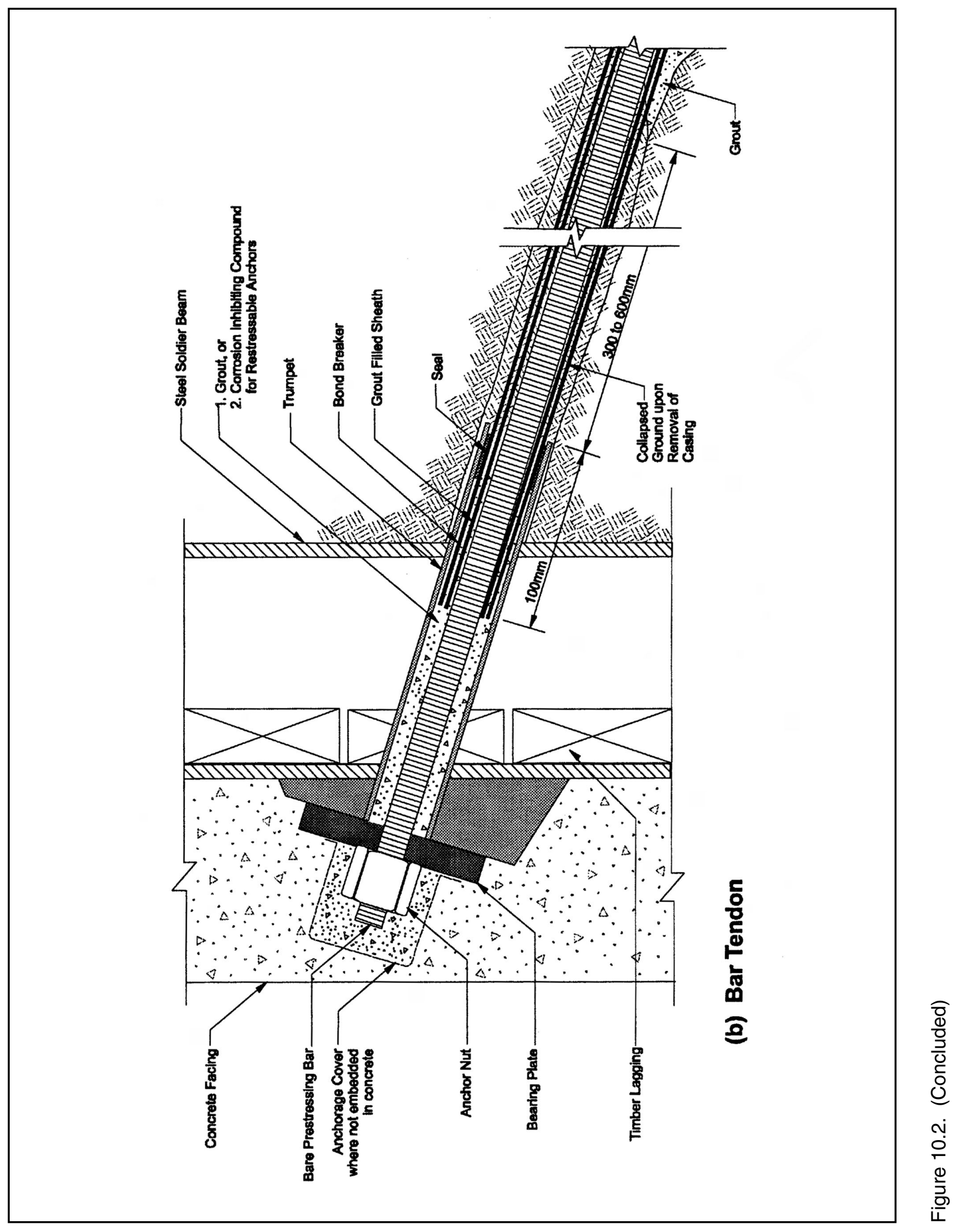




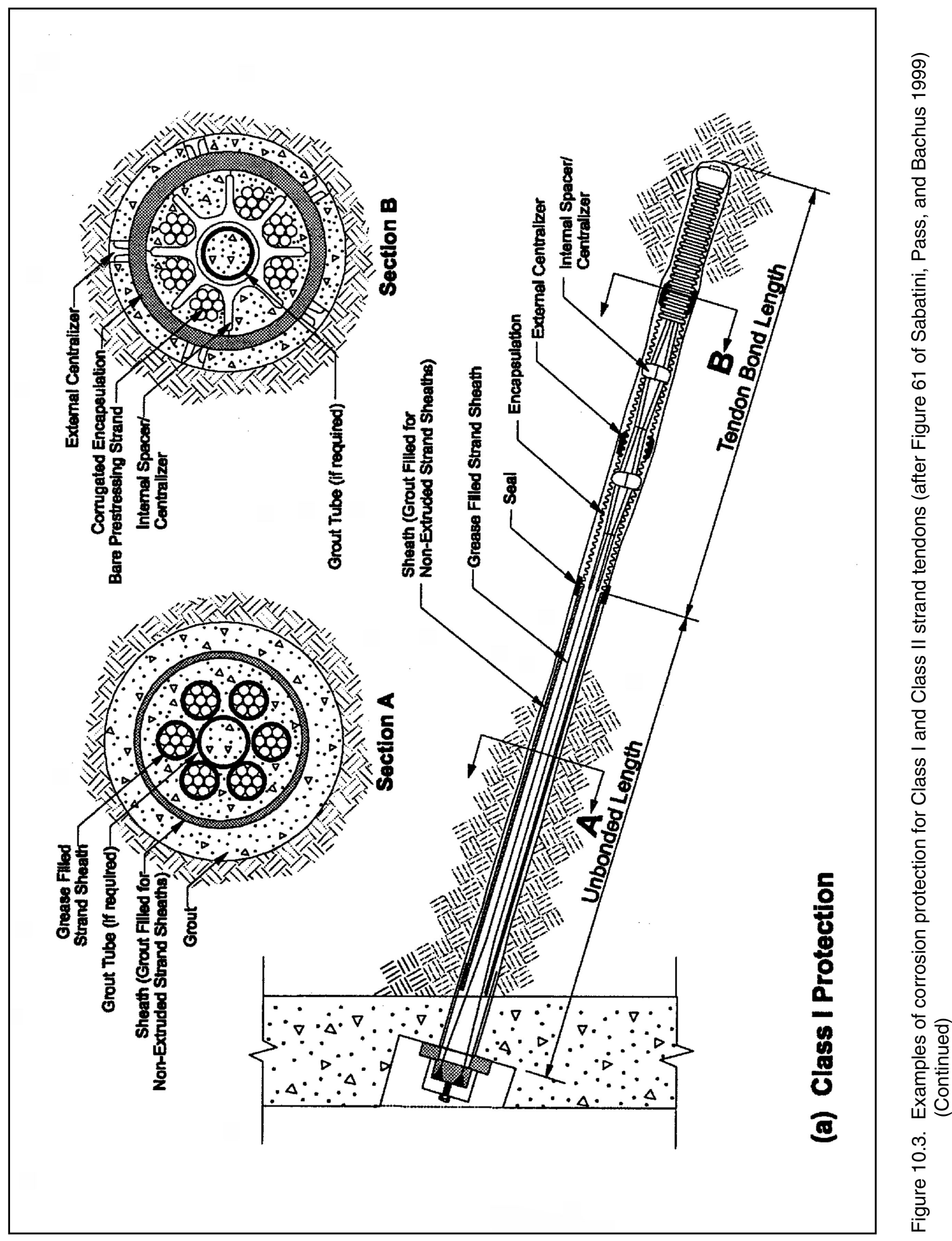




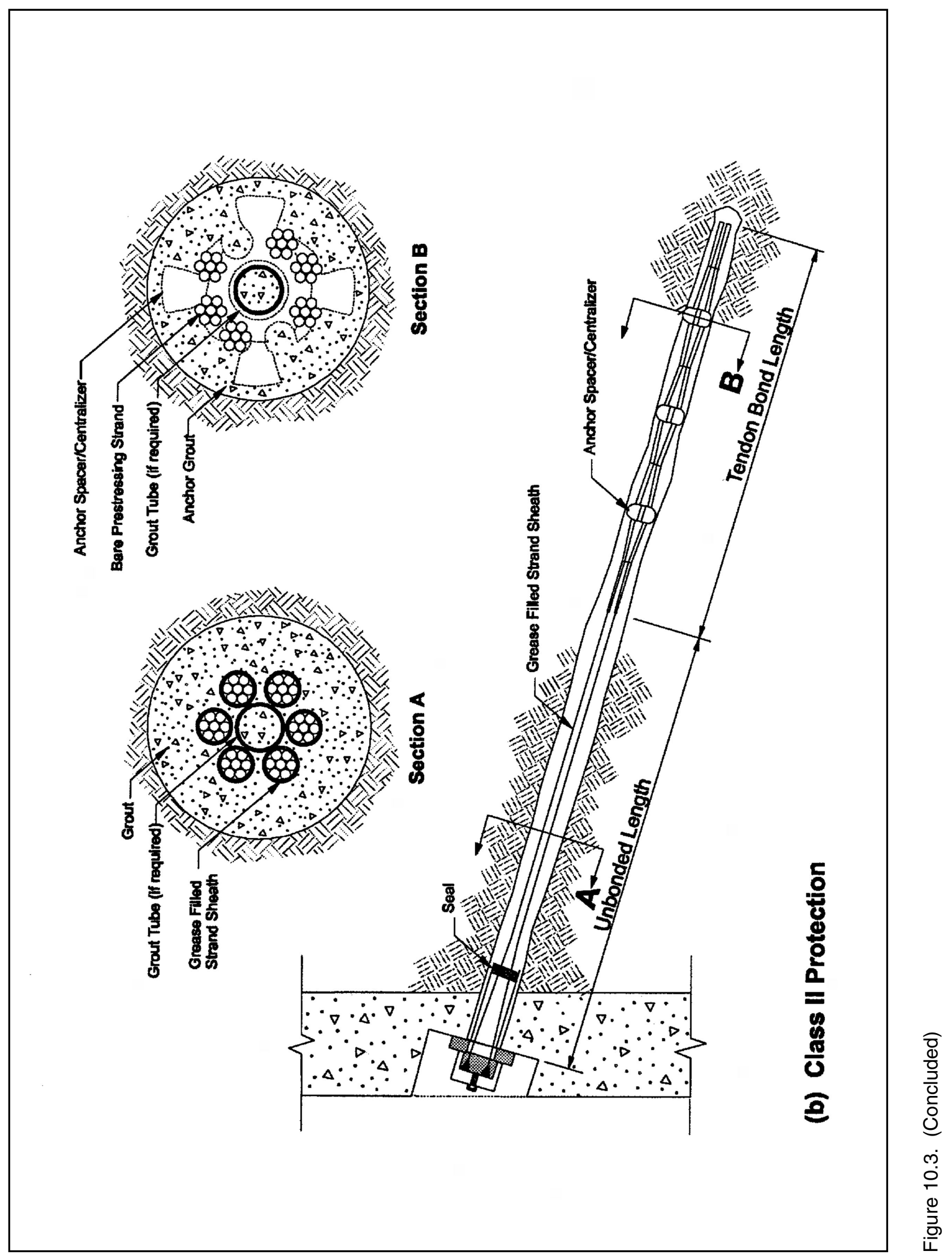




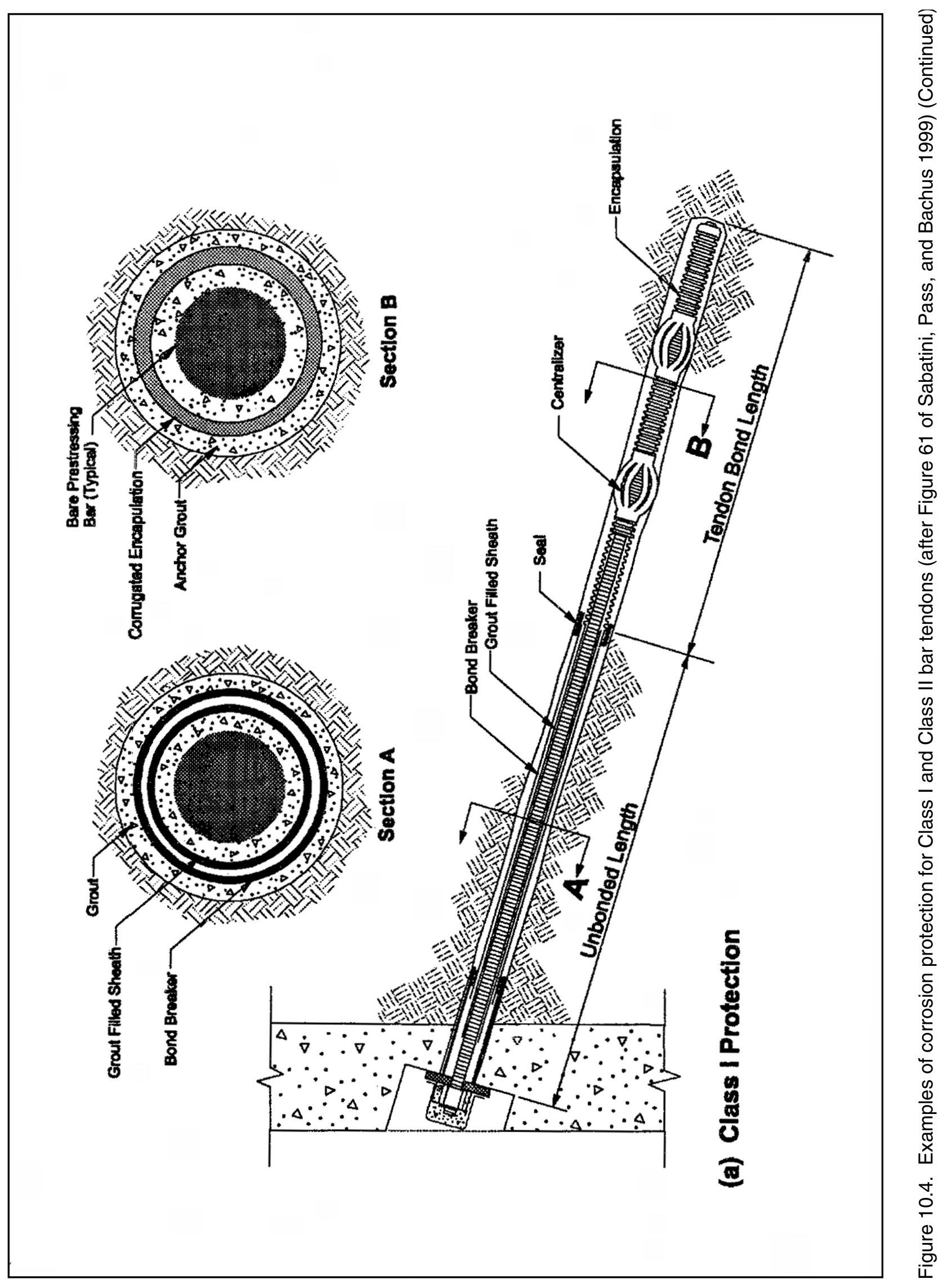




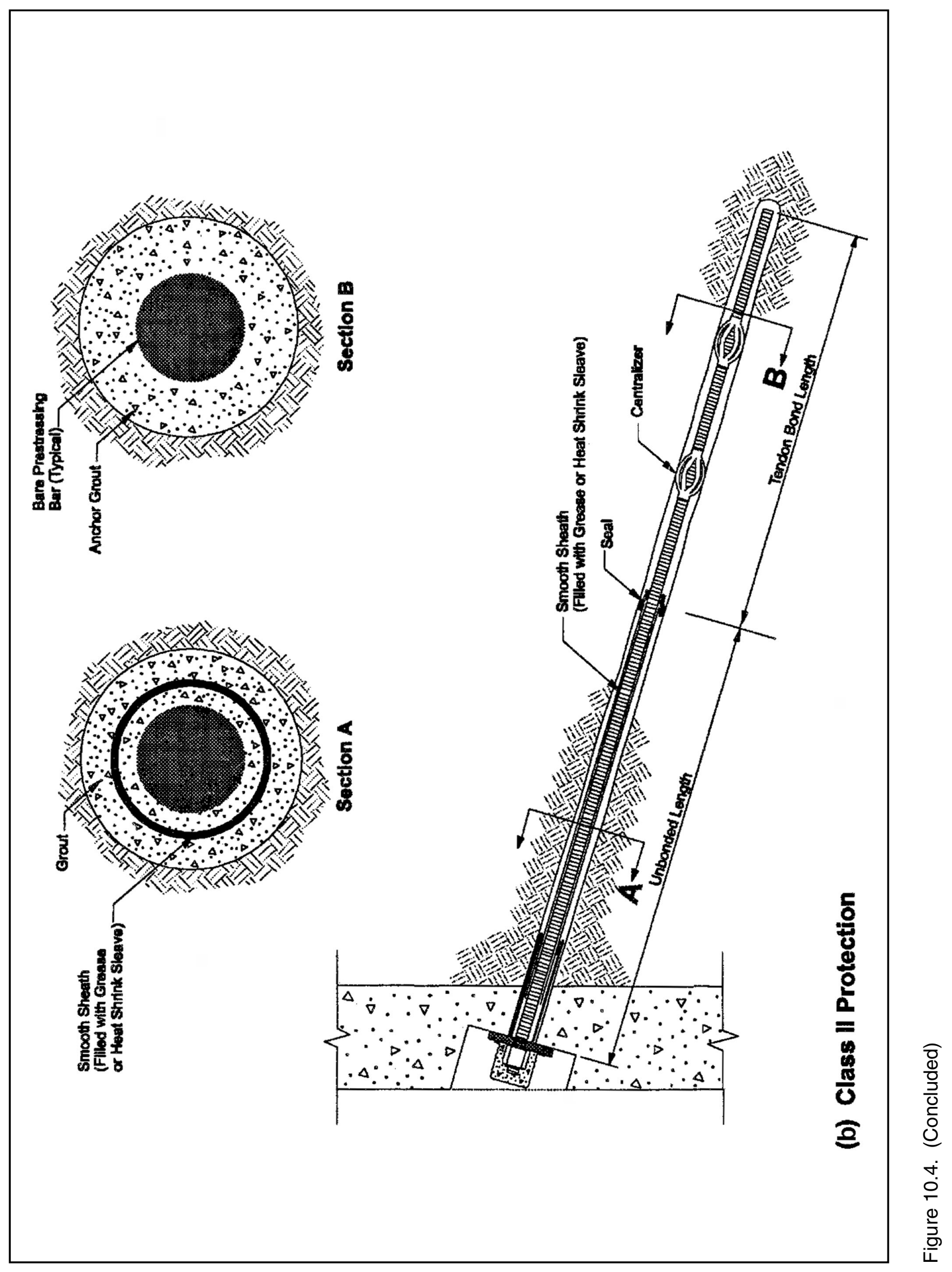


- Sheaths: Sheaths are smooth or corrugated plastic tube, smooth pipe, or extruded tubing used to protect the prestressing steel in the unbonded length. Individual strand sheaths commonly contain corrosion-inhibiting compound and are either pulled on or extruded. A tendon sheath covers all prestressing elements and is commonly pulled on and filled with grout. Smooth sheaths can function as a bondbreaker; however, corrugated sheaths require a separate bondbreaker.

- Heat-shrinkable sleeves: These sleeves are mainly used to protect couplers that connect lengths of prestressing bars and as sheaths for bar tendons.

- Encapsulations: Encapsulations are corrugated or deformed piping or tubing that protects the prestressing steel in the bond length.

- Centralizers: Centralizers are commonly made from steel or plastic and are used to support the tendon in the drill hole or within an encapsulation so that a minimum grout cover is provided around the tendon.

Three levels of minimum corrosion protection are commonly specified in U.S. practice for ground anchors. In order of descending level of protection, these are Class I protection, Class II protection, and no protection (see Table 10.1). For the anchorage and unbonded length, Class I and Class II protection assume that aggressive ground conditions exist and require that multiple barrier layers be provided for the tendon. For the bond length, Class I protection assumes that the aggressive conditions exist. This level also provides multiple barrier layers, whereas for Class II, only one barrier layer is provided. Class I and Class II protected tendons are also referred to as encapsulated tendons and grout-protected tendons, respectively. No protection against corrosion is required in ground known to be nonaggressive for anchors used for temporary support of excavation applications. The impact of aggressive ground conditions on unprotected metallic elements can be evaluated using information from Elias (1995).

10.4.3.2.2 Anchorage protection. Few anchor failures due to corrosion of the prestressing steel and/or anchorage have been reported. However, most reported anchor failures have occurred within $2 \mathrm{~m}(5 \mathrm{ft})$ of the anchorage. Careful attention should be given when installing corrosion protection at this part of the tendon. The trumpet should be attached to the bearing plate to provide a watertight seal. This seal should be strong enough to overlap the unbonded length corrosion protection by at least by $100 \mathrm{~mm}(4 \mathrm{in}$.) and should be completely filled with grout after lock-off unless restressing is anticipated.

Grout used to fill the trumpet should not escape into the unbonded length so as to slump in the trumpet. To retain the grout in the trumpet, either a seal should be provided at the bottom of the trumpet (which must function at least until the grout sets) or the trumpet should fit tightly over the unbonded length corrosion protection for a minimum of $300 \mathrm{~mm}$ (12 in.). Expansive admixtures or multigroutings may be required to ensure that the trumpet is completely filled with grout. For restressable anchors, the trumpet should be filled with a corrosioninhibiting compound and a permanent seal should be provided at the bottom of 


\begin{tabular}{|c|c|c|c|}
\hline \multicolumn{4}{|c|}{$\begin{array}{l}\text { Table } 10.1 \\
\text { Corrosion Protection Requirements (after Sabatini, Pass, and } \\
\text { Bachus 1999, Table 20) }\end{array}$} \\
\hline \multirow[b]{2}{*}{ Class } & \multicolumn{3}{|c|}{ Protection Requirements } \\
\hline & Anchorage & Unbonded length & $\begin{array}{l}\text { Tendon Bond } \\
\text { Length }\end{array}$ \\
\hline $\begin{array}{l}\text { Encapsulated } \\
\text { tendon }\end{array}$ & $\begin{array}{l}\text { 1. Trumpet } \\
\text { 2. Cover if exposed }\end{array}$ & $\begin{array}{l}\text { 1. Encapsulated tendons composed } \\
\text { of individual grease-filled extruded } \\
\text { strand sheaths with a common } \\
\text { smooth sheath. } \\
\text { 2. Encapsulated tendons composed } \\
\text { of individual grease-filled strand } \\
\text { sheaths with grout-filled smooth } \\
\text { sheath. } \\
\text { 3. Smooth bondbreaker over grout- } \\
\text { filled bar sheath. }\end{array}$ & $\begin{array}{l}\text { 1. Grout-filled } \\
\text { encapsulation or } \\
\text { 2. Fusion-bonded } \\
\text { epoxy }\end{array}$ \\
\hline $\begin{array}{l}\text { II } \\
\text { Grout-protected } \\
\text { tendon }\end{array}$ & $\begin{array}{l}\text { 1. Trumpet } \\
\text { 2. Cover if exposed }\end{array}$ & $\begin{array}{l}\text { 1. Grease-filled sheath or } \\
\text { 2. Heat-shrinkable sleeve }\end{array}$ & Grout \\
\hline
\end{tabular}

the trumpet. A restressable anchor has a special anchor head that permits measuring of lift-off throughout the service life of the structure. For corrosion inhibitorfilled trumpets, care should be taken to ensure that the seals will not leak.

The bearing plate may be protected by painting both sides with a bitumastic or other protective coating. The protective material used to paint the bearing plate should be compatible with other protective materials used above and below the bearing plate. Cast-in-place concrete facing that completely embeds the bearing plate will also provide the required protection.

Protection of the anchor head and exposed bare prestressing steel may be provided by using either a plastic or steel cover or by embedding the bare tendon in at least a 50-mm (2-in.)-thick layer of concrete during installation of the wall facing. When a cover is used, the cover should be filled with grout. For restressable anchorages, the cover should be filled with a corrosion-inhibiting compound. As with the trumpet, special care must be taken to ensure that the cover is completely filled with grout.

10.4.3.2.3 Unbonded tendon length protection. Next to the anchorage, the prestressing steel in the unbonded length is most vulnerable to corrosion. Sheaths used to protect the unbonded length should extend into the trumpet, but not so far as to come into contact with either the bearing plate or the anchor during stressing. Sheaths should be filled either with a corrosion-inhibiting compound or grout in a manner that does not leave voids. Strands should be individually coated with a corrosion-inhibiting compound, without leaving voids between wires.

For Class I protection of strands, a common smooth sheath encapsulation should be used over tendons composed of extruded grease-filled strand sheaths, or a grout-filled common smooth sheath encapsulation should be used over tendons composed of individually grease-filled strand sheaths. 
Where corrugated pipe is used as a sheath, a bondbreaker must be present. A bondbreaker is a smooth sheath used in the unbonded length that allows the prestressing steel to freely elongate during testing and stressing, and to remain unbonded to the surrounding grout after lock-off.

For Class I protection of bar tendons, the couplers must be protected. Couplers may be protected using either a corrosion-proof compound or waximpregnated cloth tape and a smooth plastic tube.

10.4.3.2.4 Tendon bond length protection. No corrosion failures have been reported when the tendons have been properly grouted (e.g., centralized and grouted in such a manner as to leave no voids around the tendon). In rock, where groundwater seepage around the tendon may be significant, drill-hole waterproofing may be necessary to ensure the grout remains in place. A watertightness test can be performed to determine the need for special waterproofing measures. If waterproofing is indicated, consolidation grout is commonly placed in the hole and redrilled approximately $18 \mathrm{hr}$ after placement. Encapsulations are used for Class I protection of the tendon bond length. Encapsulations may be pregrouted or grouted onsite prior to or after insertion of the tendon into the drill hole. Where grouted onsite, care must be taken so as to leave no voids in the grout. Centralizers are used inside the encapsulation to provide a minimum of $12 \mathrm{~mm}(0.5 \mathrm{in}$.) of grout coverage over the encapsulation.

10.4.3.2.4 Protection against stray currents. For ground anchor applications in which stray currents are present, tendons should be electrically isolated from the ground environment. Tendons that are encapsulated using a nonconductive sheath, usually plastic, along the tendon bond length and unbonded length are considered electrically isolated. However, for grout-protected or epoxy-protected tendons, the bearing plate, anchor head, and trumpet should be isolated with insulation from the wall elements. The effectiveness of the sheath to provide electrical isolation may be verified in the field by testing after installation of the tendon and prior to grouting.

10.4.3.2.5 Corrosion protection of anchors for structures subject to hydrostatic uplift. The design of a corrosion protection system for anchors used to resist uplift of a structure, or for structures in which uplift or pore pressures exist, requires careful attention to prevent water from entering the tendon through a breach in the corrosion protection.

Water entering will likely migrate up the tendon to the anchorage between the corrosion protection barrier and the prestressing elements. A Class I protection system is always required for anchors used in a tieback wall system subject to water pressures. Voids between prestressing elements and between the individual wires of a strand must be completely filled with corrosion-inhibiting compound and seals provided at the anchor head. Seals at the anchor head must remain watertight after the tendon undergoes elongation during testing or in the event of tendon elongation after lock-off. In addition, a watertight seal will often be required at the anchorage where the tendon penetrates the structure. Leakage through penetrations at the anchorage may accelerate corrosion of the anchorage. 
Seals at the anchorage are more susceptible to leakage under high water pressures. In this case, watertightness of the seals may be considered prior to construction.

\subsubsection{Selection of corrosion protection level}

10.4.4.1 General. The minimum level of corrosion protection for ground anchors should be selected considering the service life of the anchored system, the aggressivity of the ground anchor environment, the consequences of failure of the anchored system, and the cost of providing a higher level of corrosion protection.

10.4.4.2 Service life of the anchored structure. All permanent anchored wall structures require corrosion protection. If the service life of a temporary support of excavation anchor is likely to be extended due to construction delays, an evaluation should be made to determine whether to provide corrosion protection for the tendon, particularly in aggressive ground environments.

10.4.4.3 Aggressivity of the ground environment. Ground anchors in environments classified as aggressive or of known aggressivity will require the highest class of corrosion protection listed for each service life classificationClass II corrosion protection for temporary support of excavation anchors and Class I corrosion protection for permanent anchors. Tests and field observations are used to classify the aggressivity of the ground environment.

In general, ground environments may be classified as aggressive if any one of the following conditions is present in the ground or may be present during the service life of the ground anchor (PTI 1996): (1) a pH value of soil or groundwater less than 4.5 , (2) a resistivity of the ground of less than $2,000 \mathrm{ohm}-\mathrm{cm}$; (3) the presence of sulfides; (4) the presence of stray currents; or (5) buried concrete structures adjacent to the anchored system project that have suffered from corrosion or direct chemical (acid) attack. Tests are available to measure $\mathrm{pH}$ and resistivity and to identify the presence of sulfides. Tests from a nearby site can be used to evaluate the aggressivity of the site if the designer can establish that the ground conditions are similar. Otherwise, if aggressivity tests are not performed, the ground should be assumed to be aggressive.

The following ground environments are always considered aggressive: (1) soil or ground water with a low $\mathrm{pH}$; (2) saltwater or tidal marches; (3) cinder, ash, or slag fills; (4) organic fills containing humic acid; (5) peat bogs; and (6) acid mine drainage or industrial waste. Classification of ground aggressivity should consider the possibility of changes during the service life of the ground anchor, which might cause the ground to become aggressive (such as might occur near mining operation, chemical plants, or chemical storage areas).

10.4.4.4 Cost for a higher level of protection. The final criterion for selecting the minimum class of corrosion protection is the increased cost for changing from Class II protection to Class I protection. For the same tendon, Class I-protected anchors require a larger drill hole as compared to a Class IIprotected anchor. Encapsulating an anchor tendon increases the required drill-hole size, which may result in increased installation costs. In an uncased drill hole, the additional drilling costs can be small, and therefore it may be prudent to specify 
Class I protection. In a cased hole, or in rock, the additional drilling costs can be higher, and an economic analysis should be performed to determine if the higher level of protection is worth the additional cost. The increase in drill-hole diameter may result in a need to increase bearing plate dimensions, trumpet diameter, and the opening in the soldier beam to insert the tendon.

\subsubsection{Corrosion of structural steel, cement grout, and concrete}

\subsubsection{Corrosion and protection of soldier beams and sheet piles.}

Structural steels used in anchored walls (i.e., soldier beams and sheet piles) are less susceptible to failure by corrosion than are the high-strength steels used to fabricate prestressing elements for ground anchors. In most ground environments, a small loss in thickness may be expected which will not significantly reduce the strength of the structural steel. In very aggressive ground conditions, the potential for loss of thickness is significant, and the structural elements must be protected.

Below the excavation subgrade, drilled-in soldier beams are surrounded by either lean-mix or structural concrete and therefore are not considered susceptible to corrosion. Driven soldier beams and sheet piles are in direct contact with the ground and are therefore more susceptible to corrosion. Driven soldier beams and sheet piles may be protected by coatings, increasing the thickness of the steel, and using a higher strength steel (of the same dimensions) in place of lower strength steel. Coatings must be durable enough to survive driving. Coatings such as coaltar epoxy and fusion-bonded epoxy may decrease pile capacity. Guidance on the increase in steel thickness is provided in Hannigan, Goble, Thendean, Likens, and Rausche (1996).

10.4.5.2 Degradation and protection of cement grout and concrete. Although there have been no recorded anchor failures resulting from chemical attack of the cement grout of concrete, the deterioration of the grout leaves the prestressing steel vulnerable to corrosion. The primary mechanism for degradation of cement-based grout and concrete is chemical attack in high-sulfate environments, such as marshy areas and sulfate-bearing clays.

The common approach to minimizing the potential deterioration of grout and concrete in high-sulfate environments is to select a cement type based on the soluble sulfate ion $\left(\mathrm{SO}_{4}\right)$ content of the ground. For a sulfate content between 0.1 and 0.2 percent, Type II portland cement should be used, and for a sulfate content between 0.2 and 2.0 percent Type V portland cement should be used. For a sulfate content above 2.0 percent, Type $\mathrm{V}$ portland cement plus a pozzolan should be used. In addition, the rate of both sulfate and chloride attack may be significantly reduced by the use of dense grout or concrete of low permeability. The density of the grout can be controlled through placement method and the selection of watercement ratio. 


\section{Structural Integrity}

\subsection{General}

Tieback walls should be designed to sustain local damage, with the structuresoil system as a whole remaining stable. Local damage should be limited such that the wall system through remediation can be restored to a serviceable condition. This can be achieved through

- Adequate testing to ensure that the margin of safety for all tieback anchors meets design requirements.

- The use of redundant structural systems that allow, through arching action, redistribution of soil pressures to competent structural anchors and structural members adjacent to a potential damaged anchor.

- Detailing that permits the rapid replacement of failed anchors, to ensure that remediation can be carried out before tieback systems experience maximum design loadings.

- Instrumentation to permit the discovery of conditions that signal system distress.

- Progressive collapse analysis and plastic collapse mechanism analysis to ensure that system continuity and redundancies of the tieback wall systems are adequate.

\subsection{Testing of Anchor Systems}

Anchor testing is described in Weatherby (1998) and in Sabatini, Pass, and Bachus (1999). The following information is taken verbatim from Weatherby (1998):

Each ground anchor is load tested to verify that it will develop the required load-carrying capacity in accordance with testing procedures described in the contract documents. Performance, proof, or creep tests are used. The Specification for Permanent Ground Anchors [AASHTOAGC-ARTBA 1990] describes each test. Typical testing setups are shown in Weatherby (1982). Ground anchor failure criteria are based on a creep definition of failure. A creep failure occurs when the anchor movement exceeds a specified amount during a constant load hold period. Creep 
failure is different from a pullout failure. Creep failure occurs at a lower load than a pullout failure. The test load must be held constant to measure creep movements accurately. Pressure gauges are used to measure anchor loads for all three tests. Accurate pressure gauges are suitable for monitoring load during the load holds required for proof or performance tests. The following will help ensure that the load tests are run well.

- Allow the grout to gain sufficient strength. (Grout strength tests are not always performed. If a prescriptive specification is used, the owner may want to specify grout strength testing to verify the contractor has mixed a quality grout.)

- Verify that the jack and pressure gauge have been calibrated in accordance with the specifications.

- Verify the jack pressures that correspond to the test loads.

- Fill out the ground anchor test sheet before starting a test. (The Specification for Permanent Ground Anchors [AASHTO-AGCARTBA 1990] contains sample proof, performance, and creep test sheets.)

- Ensure an independent reference point is established to measure ground anchor movements.

- Ensure that the test equipment and dial gauge are aligned.

- Load test the anchors in accordance with the testing procedures described in the contract documents.

- Run performance tests on the first anchors installed on the project.

- Plot the anchor movements as the tests are performed. (Unusual behavior or errors in reading the dial gauge will be apparent if the data are plotted as the test is run.)

- Hold the ground anchor load constant during the load holds.

- Do not retest ground anchors. (Recommendations for Prestressed Rock and Soil Anchors [PTI 1996] describes a procedure that can be used to allow post-grouted anchors to be retested if they fail the acceptance criteria. The approach taken in the PTI recommendations is sound, but it has not been verified extensively by experience.)

- Recognize that ground anchor failure will occur. (Failures are most likely to occur at the beginning of the job when the contractor is refining installation techniques. If frequent failures continue, the ground installation methods may have to be modified or changed.)

- Verify that an anchor passes the acceptance criteria when the test is completed.

- Stress the anchors (lock-off) to the specified load. (The load will be between 75 and 100 percent of the design load.)

- Lift-off the anchor and verify that the desired load has been locked-off in the anchor before removing the test jack. 
It is important during tieback wall construction to perform extended creep testing to evaluate the potential creep deformation of anchors that may occur at design load levels. The additional loads that anchors adjacent to a failed anchor might experience under a possible "loss of single anchor" condition should be considered when conducting the creep test. Detailed information on extended creep testing, performance testing, proof testing, and lift-off testing can be found in Strom and Ebeling (2002b). A comprehensive testing program is required to ensure that the completed wall will meet all performance requirements.

\subsection{Redundant Systems and Detailing to Prevent Progressive Failures}

The wall itself-whether it is a discrete system (soldier beams) or a continuous system (diaphragm wall) - is generally not considered susceptible to progressive failure since, as the earth pressures increase, the wall system deflects and the pressures redistribute to the ground anchor supports (Weatherby 1998). However, progressive failure of ground anchor supports is of some concern. Load testing as described above can minimize the potential for progressive ground anchor failures. Provisions should be included in the design to facilitate the replacement of failed or poorly performing ground anchorages should the need arise. The capacity to redistribute load from those ground anchors that fail to hold their load to capable ground anchors is much greater for continuous wall systems than for discrete wall systems. This is especially true if the continuous wall system is designed for two-dimensional plate bending action. Discrete soldier beam systems can better accommodate load redistribution if provided with a cap beam designed for that purpose.

\subsection{Progressive Collapse and Plastic Collapse Mechanism Analyses}

It should be remembered that the loads selected for the original design of the ground anchors (apparent pressure diagrams) are not representative of the actual earth pressure loads at any particular stage of construction. Therefore, the anchors may have reserve capacity to accommodate the additional load resulting from failed anchor redistribution. Performance of a tieback wall system under single anchor failure conditions can be evaluated by a plastic collapse mechanism analysis. Details of the plastic collapse mechanism analysis will be presented in a future Corps report. 


\section{Contracting for Tieback Wall Systems}

\subsection{Introduction}

The purpose of this chapter is to describe contracting approaches that are commonly used in developing construction contract documents for permanent anchored systems. This information is taken verbatim from Chapter 8 of Sabatini, Pass, and Bachus (1999) and describes three contracting approaches that can be used for anchored systems: the method approach, the performance approach, and the contractor design/build approach.

The responsibilities of the owner and the contractor with respect to design, construction, and performance of the wall vary for each of these approaches. However, many years of experience in contracting for anchored walls has shown that the owner should not specify the installation details of the anchor. All contracting approaches should use performance-based acceptance criteria for the anchors. Contracting approaches for anchored walls and other wall systems are also described elsewhere (e.g., Nicholson and Bruce 1992; Deaton 1994; Byrne, Cotton, Porterfield, Wolschlag, and Ueblacker 1998; Weatherby 1998).

- Method approach: Method specifications are used for owner or materialsupplier designs. In the contract documents, wall construction materials and the execution of construction are explicitly specified except for the selection of the anchor type and the anchor installation details. This contracting approach is discussed further in Section 12.2.

- Performance approach: This type of contracting specification, also referred to as an "end result" specification, uses approved or generic wall systems or components. Included in the contract documents are lines and grades, as well as specific geometric, design, and performance criteria. For this approach, the contractor submits project-specific design calculations and plans for owner review in conjunction with normal working drawing submittals. This contracting approach is discussed further in Section 12.3.

- Contractor design/build approach: This type of contracting approach is similar to the performance approach, except the responsibility for design, construction, and performance of the completed anchored wall is placed 
solely on the specialty contractor. This method requires a strict prequalification process as part of the selection of the specialty contractor.

Performance-based submittals should be provided to the owner at key times during design and construction. This contracting approach is discussed further in Section 12.4.

Each of these contracting approaches may be used for an anchored wall, if properly implemented. Often, the approach will be selected based on the experience of the owner and his engineering consultants with anchored systems, the complexity of the project, the availability of specialty contractors or material suppliers, and the local highway agency philosophy with respect to contracting methods. Regardless of which contracting approach is chosen for a specific project, it is highly desirable that each owner develop a formal policy with respect to design and contracting of anchored systems. The general objectives of such a policy are to

- Obtain local highway agency uniformity in selection of anchored systems and other earth-retaining system alternatives.

- Establish standard policies and procedures for technical review and acceptance of proprietary and generic anchored systems and other earthretaining systems.

- Establish internal agency responsibility for the acceptance of new anchored systems and other earth-retaining systems and/or components, and for plan preparation, design review, and construction control.

- Develop uniform design and performance criteria standards and construction and material specifications for anchored systems and other earthretaining systems.

- Establish guidelines for the selection of method, performance, or contractor design/build contracting approaches.

\subsection{Method Contracting Approach}

\subsubsection{Introduction}

The method contracting approach includes the development of a detailed set of plans and material and construction specifications for the bidding documents. However, the selection and installation of the anchors should be the responsibility of the contractor. The contract documents should only establish minimum dimensions for drill-hole diameter, unbonded length, and bond length. The contractor should select the necessary anchor installation dimensions and techniques to successfully pass the acceptance tests. In no case should the owner specify the installation details for the anchors. The advantage of the method approach is that the complete design and specifications are developed and reviewed over an extended design period. This approach enables the owner's engineers to examine various 
options during design, but requires an engineering staff trained in all areas of earth-retaining system technology. The method contracting approach is best suited where the owner has developed significant experience in design and construction of anchored walls.

A disadvantage of the method approach is that, for alternate bids, more sets of designs must be reviewed. Therefore, agency resources must be expended even though only one wall system will be constructed. Another disadvantage is that agency personnel may be unfamiliar with newer and potentially more costeffective systems and may not consider them during the design stage. Similarly, proprietary equipment and methods used by particular anchored-system contractors may be unfamiliar to agency personnel and will therefore not be considered.

When a method contracting approach is adopted, the owner and the owner's inspector are fully responsible for the design and performance of the anchored wall, provided the contractor has constructed the system in full accordance with the specifications. In the event that changes are necessary, the owner must be prepared to direct and pay for the contractor's work.

\subsubsection{Contract documents for method approach}

The contract documents in the method approach consist of drawings, specifications, and bidding items and quantities. The contract can be bid on a lump sum basis or following a detailed unit price list. Drawings prepared using the method approach should typically include at least the following items:

- Horizontal alignment of the wall identified by stations and offset from the horizontal control line to the face of the wall and all appurtenances that affect construction of the wall.

- Elevation at the top and bottom of the wall, beginning and end stations for wall construction, horizontal and vertical positions at points along the wall, and locations and elevations of the final ground line.

- Cross sections showing limits of construction, existing underground interferences such as utilities or piles supporting adjacent structures, any backfill requirements, excavation limits, as well as mean high water level, design high water level, and drawdown conditions, if applicable.

- Notes required for construction including general construction procedures and all construction constraints such as staged construction, vertical clearance, right-of-way limits, construction easements, noise and air quality requirements, etc.

- Typical sections and special details.

- Dimensional and alignment tolerances during construction. 
- All details for connections to traffic barriers, copings, parapets, noise walls, and attached lighting.

- Payment limits and quantities.

In addition to the items described above, other items specific to anchored walls should be included in the bidding documents, including

- Size, type, location, method of installation, and minimum embedment depth of all wall elements.

- Thickness of timber lagging and all details for facing installation, thickness, size, type, and finish, and final facing connections to soldier piles or sheet piles and/or walers.

- Location of all ground anchors and structural connection details for the anchor to the sheet-pile, soldier beam, or waler system.

- Corrosion protection requirements or details for the anchorage, the unbonded length, and the bond length.

- Required ground anchor capacity, inclination, minimum unbonded length, and minimum anchor bond length for each anchor.

- Requirements or details for methods and frequency of proof, performance, extended creep, and lift-off testing of anchors, ground anchor acceptance criteria, and required lock-off load.

\subsection{Performance Contracting Approach}

\subsubsection{Introduction}

For the performance contracting approach, the owner establishes the scope of work and prepares drawings showing the geometric requirements of the anchored wall, design loadings, material specifications, or components that may be used, performance requirements, and any instrumentation or monitoring requirements.

The performance approach offers several benefits over the method approach when used with appropriate specifications and prequalification of suppliers, specialty contractors, and materials. Design of the structure is the responsibility of the contractor and is usually performed by a trained and experienced contractor or engineering consultant. This enables engineering costs and manpower requirements for the owner to be decreased, since the owner's engineer is not preparing a detailed design, and transfers some of the design cost to construction.

The disadvantage of the performance approach is that the owner's engineers may not be experienced with anchored system technology and, therefore, may not be fully qualified to review and approve the wall design and any construction 
modifications. Newer and potentially more cost-effective methods and equipment may be rejected due to the lack of confidence of owner personnel to review and approve these systems.

Three principal methods have been used to implement the performance approach for anchored walls. These methods, referred to as pre-bid wall design, pre-bid typical section design, and post-bid design, are described in subsequent sections. Differences between these methods are associated with the required time to perform the design. Other methods such as two-phase bidding and negotiated work proposals have been used for specialized anchor projects (see Nicholson and Bruce 1992).

\subsubsection{Implementing performance contracting approach}

12.3.2.1 Pre-bid wall design. Contract documents for pre-bid wall designs are prepared to allow for various retaining wall alternatives. With this method, the owner contacts specialty contractors and informs them that a retaining wall is being proposed for a site. The owner requests that the contractors prepare detailed wall designs prior to the advertisement of the bid. The designs are based on owner-provided line and grade information, geotechnical and subsurface information, and design requirements. Approved designs are then included in the bid documents. This approach allows the owner to review design details based on submittals from several contractors. Because of the detail that must be provided with this type of a submission, only those contractors who have significant expertise and experience in anchored systems are likely to prepare the required submission. The owner should prepare and include a generic wall system design in the bid documents to enable general contractors to decide whether they want to use the generic design or a design from a specialty contractor.

12.3.2.2 Pre-bid typical section design. With pre-bid typical section design, schematic or conceptual plans are developed by prequalified specialty contractors based on geometric and performance requirements specified by the owner.

Sufficient detail must be provided by the specialty contractor to enable the owner to judge whether the approach of the contractor is acceptable. Contractors will typically exclude details they believe are unique to their design. The advantage of this approach compared to pre-bid wall design is that specialty contractors are more likely to submit their solutions for review and inclusion in the bid documents. With this approach, only limited preparation effort is required by the contractor, and development of a detailed design and working drawings is necessary only if he is the successful bidder.

The disadvantage of this approach is that total project requirements are less well defined and may lead to misunderstandings and claims. In cases where the general contractor will not be constructing the anchored system, the apparent lack of detail using this approach may result in problems during construction because the general contractor does not fully understand the design. For example, the approved tolerances on soldier beam installation may require more concrete for the facing than the general contractor anticipated (Deaton 1994). 
12.3.2.3 Post-bid wall design. Like pre-bid wall design and pre-bid typical section design, the post-bid wall design approach allows for various prequalified contractor-designed wall alternates. In the bid documents, each wall and acceptable alternates are identified. Design requirements for each wall type are contained in the special provisions or standard agency specifications. General contractors receive bids from prequalified specialty contractors and subsequently select a specialty contractor-prepared wall design and wall price to include in their bid. Once the contract is awarded, if the general contractor decides to build the anchored system, he then requests that the selected specialty contractor prepare detailed design calculations and a complete set of working drawings for owner review and approval. Upon approval, the walls are built in accordance with the working drawings. When an owner uses this type of contract, he benefits from the experience of the wall contractors or supplier. However, he does not have as much control over the finished product as when he requires pre-bid approval of the working drawings. Also, since the general contractor wants to minimize risk, he will likely not select an alternate design unless the construction cost savings are significant.

\subsubsection{Contract documents for performance approach}

Regardless of which performance approach is used, the owner must prepare and include as part of the contract documents geometric and site data, design guidelines, and performance requirements. Also, for performance specifications, an instrumentation and monitoring program is usually included as part of the design. For anchored systems, the monitoring program will typically include requirements with respect to performance, proof, and extended creep tests. Minimum levels of instrumentation to be used by the contractor and threshold values against which the monitoring data will be evaluated are also included. Required information is listed below.

\section{Geometric and site data}

- Horizontal alignment of the wall identified by stations and offset from the horizontal control line to the face of the wall and all appurtenances that affect construction of the wall.

- Elevation at the top and bottom of the wall, beginning and end stations for wall construction, horizontal and vertical positions at points along the wall, and locations and elevations of the final ground line.

- Cross sections showing limits of construction, any backfill requirements, excavation limits, as well as mean high water level, design high water level, and drawdown conditions, if applicable.

- All construction constraints such as staged construction limitations, vertical clearance, right-of-way limits, construction easements, etc.

- Location of utilities, signs, etc., and any loads that may be imposed by these appurtenances. 
- Data obtained as part of a subsurface investigation and geotechnical testing program.

\section{Design guidelines}

- Reference to specific governing sections of appropriate agency design manuals (materials, structural, hydraulic, and geotechnical), construction specifications, and special provisions. If none such guidelines are available, reference to current AASHTO Standard Specifications may be used.

- Magnitude, location, and direction of external loads due to bridges, overhead signs and lights, and traffic surcharges.

- Limits and requirements of drainage features beneath, behind, above, or through the structure.

- Seismic design requirements.

- Minimum factors of safety for potential failure mechanisms such as overall stability, pullout failure of the anchor, rupture of the anchor tendon, axial and lateral wall capacity, etc.

- Geotechnical design parameters such as friction angle, cohesion, and unit weight, as well as electrochemical properties of the soils to be used.

- Type, size, and architectural treatment of permanent facing.

\section{Performance requirements}

- Design life for the structure and corrosion protection requirements.

- All testing requirements and acceptance criteria for ground anchors.

- Tolerable horizontal and vertical movements of the structure and methods of measuring these movements when movement-sensitive structures exist behind the wall. In general, the owner should consider the need for movement control when structures are located within a horizontal distance from the top of the wall equal to one-half the wall height.

- Permissible range of variation in groundwater levels and methods of groundwater level measurement. In general, the owner should consider the need for groundwater control when existing structures are located near the wall. 


\subsubsection{Review and approval}

Where a performance contracting approach is used, the review process may be made prior to or after the bid, depending on the method used. The evaluation by agency structural and geotechnical engineers must be rigorous and should, as a minimum, consider the following items:

- Conformance to the project line and grade.

- Conformance of the design calculations to the agency standards or special provisions or codes such as the current AASHTO Standard Specifications with respect to design methods.

- Corrosion protection details.

- Development of design details at obstructions such as drainage structures or other appurtenances.

- External and internal drainage features and details.

- Architectural treatment of the wall face.

- Monitoring methods as required by the performance specifications.

- Field testing program details for evaluating the capacity of the anchors.

\subsection{Contractor Design/Build Approach}

For the contracting approaches previously described, the owner and contractor share responsibility in the design and construction of the anchored system. With the contractor design/build method, the owner outlines the project requirements, obtains complete subsurface and geotechnical information, and provides construction quality assurance. The specialty contractor is responsible for the complete design, construction, and performance of the anchored system. A design/build proposal may be submitted either before the bid advertisement (pre-bid) or after the contract award (post-bid). This method is most often used for securing bids on temporary ground anchor projects, and has been used on permanent anchored wall projects. The key elements for a successful contract are communication of basic design concepts to the owner and the joint development of a quality assurance plan prior to construction.

\subsection{Recommendations}

The three contracting approaches described above have been used to contract ground anchor and anchored system work. Regardless of the contracting approach selected, a performance-based approach should be used for the construction details of the anchor. Specialty contractors have developed various anchored 
systems, construction equipment, and construction methods that are appropriate for specific soil/site conditions. It is in the competitive interest of the specialty contractor to remain current on latest innovations in the field. Public agencies can, therefore, benefit from these innovations by specifying anchor performance requirements rather than specific components of the anchored system. The owner must specify certain minimum requirements such as corrosion protection for the ground anchors and other components, minimum unbonded and bond length, and inclination and total anchor length based on right-of-way restrictions and acceptance/rejection criteria for the anchor system and anchor system components. It is recommended that construction details be the sole responsibility of the contractor.

Prequalification of specialty contractors is essential. Prequalification should be based on successful experience in design and construction of anchored systems in similar ground conditions and in the region where the proposed anchored system is to be constructed. 


\section{References}

AASHTO. (1996). "The standard specification for highway bridges," 16th ed. American Association of State Highway and Transportation Officials, Washington, DC.

AASHTO-AGC-ARTBA. (1990). "Specification for permanent ground anchors," Joint Committee Task Force 27, American Association of State Highway and Transportation Officials-Associated General Contractors of America-American Road and Transportation Builders Association, Washington, DC.

ANSI/ASCE. (1995). "Minimum design loads for buildings and other structures," Publication 7-95, American National Standards Institute/American Society of Civil Engineers, Reston, VA.

ASCE. (1997). "Guidelines of engineering practice for braced and tied-back excavations," Geotechnical Special Publication 74, American Society of Civil Engineers, Reston, VA.

ASCE/SEI. (2000). "Effective analysis of diaphragm walls," Joint Technical Committee on Performance of Structures During Construction, American Society of Civil Engineers/Structural Engineering Institute, Reston, VA.

ASTM. (1997). Annual book of ASTM standards. American Society for Testing and Materials, Philadelphia, PA.

Azene, M., and Ebeling, R. M. (2002). "Simplified procedures used for the design of flexible anchored tieback walls," ERDC/ITL Technical Report (in publication), U.S. Army Engineer Research and Development Center, Vicksburg, MS.

Broms, B. B. (1965). "Design of laterally loaded piles," Journal of the Soil Mechanics and Foundations Division (ASCE) 91(SM3), Proceedings Paper 4342, 79-99.

Burmister, D. M. (1962). "Physical, stress-strain, and strength responses of granular soils," Special Technical Publication No. 322, 67-97. American Society for Testing and Materials, Philadelphia, PA. 
Byrne, R. J., Cotton, D., Porterfield, J., Wolschlag, C., and Ueblacker, G. (1998). "Manual for design and construction monitoring of soil nail walls," Report FHWA-DP-96-69F, Federal Highway Administration, Washington, DC.

Cheney, R. S. (1990). "DP-68 Permanent ground anchors; 2, Field demonstration project summaries," Report FHWA-DP-90-068-003, Federal Highway Administration, Washington, DC.

Clough, G. W., and Duncan, J. M. (1969). "Finite element analysis of Port Allen and Old River Locks," Report TE-69-3, U.S. Army Engineer Waterways Experiment Station, Vicksburg, MS.

Clough, G. W., and Duncan, J. M. (1971). "Finite element analysis of retaining wall behavior," Journal of the Soil Mechanics and Foundations Division (ASCE) 97(SM12), 1657-73.

. (1991). "Chapter 6, Earth pressures,” Foundation engineering handbook, 2nd ed., H. Y. Fang, ed. Van Nostrand Reinhold, NY, 223-35.

Clough, W. G., and O'Rourke, T. D. (1990). "Construction induced movements of in situ walls," Geotechnical Special Publication 25, American Society of Civil Engineers, Reston, VA.

Dawkins, William P. (1994a). "User's guide: Computer program for Winkler soilstructure interaction analysis of sheet-pile walls (CWALSSI)," Instruction Report ITL-94-5, U.S. Army Engineer Waterways Experiment Station, Vicksburg, MS.

(1994b). "User's guide: Computer program for analysis of beamcolumn structures with nonlinear supports (CBEAMC)," Instruction Report ITL-94-6, U.S. Army Engineer Waterways Experiment Station, Vicksburg, MS.

Deaton, H. J. (1994). "Contracting for tieback walls on a design-build basis." Serviceability of earth retaining structures, Geotechnical Special Publication 42, 17-23. American Society of Civil Engineers, Reston, VA.

Desai, C. S., Drumm, E.C., and Zaman, M. M. (1985). "Cyclic testing and modeling of interfaces," Journal of Geotechnical Engineering (ASCE) 111(6), 793-815.

Desai, C. S., Muqtadir, A., and Scheele, F. (1986). "Interaction analysis of anchorsoil systems," Journal of Geotechnical Engineering (ASCE) 112(5), 537-53.

Duncan, J. M., and Buchignani, A. L. (1975). "An engineering manual for slope stability studies," University of California Berkeley, Department of Civil Engineering, Geotechnical Engineering, Berkeley, CA. 
Duncan, J. M., Clough, G. W., and Ebeling, R. M. (1990). "Behavior and design of gravity earth retaining structures," Proceedings, ASCE specialty conference on design and performance of earth retaining structures, Geotechnical Special Publication 25. American Society of Civil Engineers, Reston, VA. 252-77.

Ebeling, R. M., and Morrison, E. E. (1992). "The seismic design of waterfront retaining structures,” Technical Report ITL-92-11, U.S. Army Engineer Waterways Experiment Station, Vicksburg, MS.

Ebeling, R. M., and Mosher, R. L. (1996). "Red River U-frame Lock No. 1 backfill-structure-foundation interaction," Journal of Geotechnical Engineering (ASCE) 122(30), 216-25.

Ebeling, R. M., and Wahl, R. E. (1997). "Soil-structure-foundation interaction analysis of new roller-compacted concrete North Lock Wall at McAlpine Locks," Technical Report ITL-90-5, U.S. Army Engineer Waterways Experiment Station, Vicksburg, MS.

Ebeling, R. M., Mosher, R. L. Abraham, K., and Peters, J. F. (1993). "Soil structure interaction study of Red River Lock and Dam No. 1 subjected to sediment loading," Technical Report ITL-93-3, U.S. Army Engineer Waterways Experiment Station, Vicksburg, MS.

Ebeling, R. M., Duncan, J. M., and Clough, G.W. (1990). "Methods of evaluating the stability and safety of gravity earth retaining structures founded on rockPhase 2 study,” Technical Report ITL-90-7, U.S. Army Engineer Waterways Experiment Station, Vicksburg, MS.

Ebeling, R. M., Pace, M. E., and Morrison, E. E. (1997). "Evaluating the stability of existing massive concrete structures founded on rock," Technical Report REMR-CS-54, U.S. Army Engineer Waterways Experiment Station, Vicksburg, MS.

Ebeling, R. M., Peters, J. F., and Clough, G.W. (1992). “User's guide for the incremental construction Soil-Structure Interaction Program SOILSTRUCT," Technical Report ITL-90-6, U.S. Army Engineer Waterways Experiment Station, Vicksburg, MS.

Ebeling, R. M., Peters, J. F., and Mosher, R. L. (1997). "The role of non-linear deformation analysis in the design of a reinforced soil berm at Red River U-frame Lock No. 1," International Journal for Numerical and Analytical Methods in Geomechanics 21, 753-87.

Elias, V. (1995). "Corrosion/degradation of soil reinforcements for mechanically stabilized walls and reinforced soil slopes," Report FHWA-SA-96-072, Federal Highway Administration, Washington, DC.

Fang, H., ed. (1991). Foundation engineering handbook. 2nd ed., Van Nostrand Reinhold, New York. 
FHWA. (1999). "Training course in geotechnical and foundation engineering: Rock slopes-Participants manual," Federal Highway Administration, McLean, VA.

Gómez, J. E., Filz, G.M., and Ebeling, R. M. (2000). "Development of an improved numerical model for concrete-to-soil interfaces in soil-structure interaction analysis; Report 2, Final study," Technical Report ITL-99-1, U.S. Army Engineer Waterways Experiment Station, Vicksburg, MS.

Goodman, R. E., Taylor, R. L., and Brekke, T. L. (1968). "A model for the mechanics of jointed rock," Journal of the Soil Mechanics and Foundations Division (ASCE) 94(SM3), 637-59.

Haliburton, T. A. (1987). "Soil structure interaction: Numerical analysis of beams and beam-columns," Technical Publication No. 14, School of Civil Engineering, Oklahoma State University, Stillwater, OK.

Hannigan, P. I., Goble, G. G., Thendean, G., Likins, G. E., and Rausche, F. (1996). "Design and construction of driven pile foundations; I," Report FHWA-HI-97-013, Federal Highway Administration, Washington, DC.

Headquarters, Department of the Army. Engineer Manuals. Washington, DC. EM 1110-1-1804, Geotechnical Investigations (1984).

EM 1110-1-1905, Bearing Capacity of Soils (1992).

EM 1110-1-1906, Soil Sampling (1996).

EM 1110-1-2908, Rock Foundations (1994).

EM 1110-2-1906, Laboratory Soils Testing

EM 1110-2-2104, Strength Design for Reinforced-Concrete Hydraulic Structures (1992).

EM 1110-2-2105, Design of Hydraulic Steel Structures (1994).

EM 1110-2-2502, Retaining and Flood Walls (1989).

EM 1110-2-2504, Design of Sheet Pile Walls (1994).

EM 1110-2-2906, Design of Pile Foundations (1991).

Henkel, D. J. (1971). "The calculation of earth pressures in open cuts in soft clays," ASCE Journal 6(4), 14-15.

Johnson, E. (1953). "The effects of restraining boundaries on the passive resistance of sand; Results of a series of tests with a medium-scale testing apparatus," M.S. thesis, Princeton University, New Jersey.

Kerr, W. C., and Tamaro G. J. (1990). "Diaphragm walls—Update on design and performance." Proceedings, ASCE specialty conference on design and performance of earth retaining structures, Geotechnical Special Publication 25. American Society of Civil Engineers, Reston, VA.

Lee, P. A., Kane, W. F., Drumm, E. C., and Bennett, R. M. (1989). "Investigation and modeling of soil-structure interface properties." Foundation engineering: Current principles and practice, Geotechnical Special Publication 22, 580-87. American Society of Civil Engineers, Reston, VA. 
Liao, S. C., and Neff, Thom L. (1990). "Estimating lateral earth pressure for design of excavation supports." Proceedings, ASCE specialty conference on design and performance of earth retaining structures, Geotechnical Special Publication 25. American Society of Civil Engineers, Reston, VA.

Long, J. H., Weatherby, D. E., and Cording, E. J. (1998). "Summary report of research on permanent ground anchor walls; I, Current practice and limiting equilibrium analyses," Report FHWA-RD-98-065, Federal Highway Administration, McLean, VA.

Matsui, T., and San, K. C. (1979). "An elastoplastic joint element with its application to reinforced slope cutting," Soils and Foundations 29(3), 95-104.

Maurseth, J., and Sedey, J. S. (1991). "Slurry constructed diaphragm guard wall, Bonneville Navigation Lock," Structural Engineering Conference, U.S. Army Corps of Engineers, Washington, DC.

Meyerhof, G. G. (1974). "Ultimate bearing capacity of footings on sand overlying clay," Canadian Geotechnical Journal 11, 223-29.

Mosher, R. L., and Knowles, V. R. (1990). "Finite element study of tieback wall for Bonneville Navigation Lock," Technical Report ITL-90-4, U.S. Army Engineer Waterways Experiment Station, Vicksburg, MS.

Mueller, C. G., Long, L. H., Weatherby, D. B., Cording, B. L., Powers, W. F., III, and Briaud, J-L. (1998). "Summary report of research on permanent ground anchor walls; III, Model-scale wall tests and ground anchor tests," Report FHWA-RD-98-067, Federal Highway Administration, McLean, VA.

Munger, D. F., Jones, P. T., and Johnson, J. (1991). "Temporary tieback wall, Bonneville Navigation Lock," Structural Engineering Conference, U.S. Army Corps of Engineers, Washington, DC.

Murchison, J. M., and O'Neill, M. W. (1984). “An evaluation of $P$-y relationships in cohesionless soils.” Proceedings, ASCE conference on analysis and design of pile foundation, San Francisco. American Society of Civil Engineers, Reston, VA.

NAVFAC. (1982). "Foundations and earth structures," Design Manual 7.2, Department of the Navy, Naval Facilities Engineering Command, Alexandria, VA.

Nicholson, G. A. (1983). "Design of gravity dams on rock foundations: Sliding stability assessment by limit equilibrium and selection of shear strength parameters," Technical Report GL-83-13, U.S. Army Engineer Waterways Experiment Station, Vicksburg, MS. 
Nicholson, P. J., and Bruce, D. A. (1992). "Opportunities and constraints for the innovative geotechnical contractor." Excavation and support for the urban infrastructure, Geotechnical Special Publication 33, 46-44. American Society of Civil Engineers, Reston, VA.

O’Neill, M. W., and Murchison, J. M. (1983). "An evaluation of $P-y$ relationships in sand," Research Report GT-DF02-83, University of Houston, Houston, TX.

O’Neill, M. W., and Reese, L. C. (1999). "Drilled shafts: Construction procedures and design methods," Report FHWA-SA-99-019, Federal Highway Administration, Washington, DC.

Peck, R. B. (1969). "Deep excavations and tunneling in soft ground--State of art report." Proceedings, 7th international conference on soil mechanics and foundation engineering. Mexico City, Mexico, 225-90.

Peck, R. B., and Davisson, M. T. (1962). "Discussion of 'Design and stability considerations for unique pier,' by J. Michalos and D. P. Billington," Transactions of the ASCE 127, 413-24.

Peck, R. B., Hanson, W. E., and Thornburn, T. H. (1974). Foundation engineering. John Wiley and Sons, New York.

Petersen, M. S., Kulhway, F. H., Nucci, L. R., and Wasil, B. A. (1976). "Stressdeformation behavior of soil-concrete interfaces," Contract Report B-49, prepared for Niagara Mohawk Power Corporation, Syracuse, NY.

Pfister, P., Evers, G., Guillaud, M., and Davidson, R. (1982). "Permanent ground anchors-Soletanche design criteria," Report FHWA-RD-81-150, Federal Highway Administration, McLean, VA.

Post-Tensioning Institute (PTI). (1996). "Recommendations for prestressed rock and soil anchors," 3rd ed., Phoenix, AZ.

Ratay, R. T. (1996). Handbook of temporary structures in construction. McGrawHill, New York.

Reese, L. C. (1958). 'Discussion of 'Soil modulus for laterally loaded piles,' by McClelland and Focht," Transactions of the ASCE 123, 1071-74.

Reese, L. C., and O'Neill, M. W. (1988). "Drilled shafted: Construction procedures and design methods," FHWA Report HI-88-042, Federal Highway Administration, Washington, DC.

Reese, L. C., Cox, W. R., and Koop, F. D. (1975). "Field testing and analysis of laterally loaded piles in sand." Proceedings, 5th offshore technology conference, Vol II, Paper OTC 2080. Houston, TX. 
Sabatini, P. I., Pass, D. G., and Bachus, R. C. (1999). "Ground anchors and anchored systems," Geotechnical Engineering Circular No. 4, FHWA-SA-99015, Federal Highway Administration, Washington, DC.

Schmertmann, J. H. (1978). "Guidelines for cone penetration test performance and design," Report No. FHWA-TS-78-209, Federal Highway Administration, McLean, VA.

Strom, R. W., and Ebeling, R. M. (2002a). "Simplified procedures used for the design of stiff anchored tieback walls," ERDC/ITL Technical Report (in publication), U.S. Army Engineer Research and Development Center, Vicksburg, MS.

Strom, R. W., and Ebeling, R. M. (2002b). "Methods used in tieback wall design and construction to prevent local anchor failure, progressive anchorage system failure, and ground mass stability failure," ERDC/ITL Technical Report (in publication), U.S. Army Engineer Research and Development Center, Vicksburg, MS.

Tamaro, G. J. (1990). "Slurry wall design and construction." Proceedings, ASCE specialty conference on design and performance of earth retaining structures. Geotechnical Special Publication 25. American Society of Civil Engineers, Reston, VA.

Terzaghi, K. (1934). "Large retaining wall tests; I, Pressure of dry sand," Engineering News-Record III, 136-40.

. (1936). "A fundamental fallacy in earth pressure calculations," Boston Society of Civil Engineers (Apr) 71-88.

Terzaghi, K. (1954). “Anchored bulkheads,” Transactions of the American Society of Civil Engineers 119, 1243-1324.

. (1955). "Evaluation of coefficient of subgrade reaction," Geotechnique 5, 297-326.

Terzaghi, K., and Peck, R. B. (1967). Soil mechanics in engineering practice. John Wiley and Sons, New York.

Terzaghi, K., Peck, R. B., and Mesri, G. (1996). Soil mechanics in engineering practice. 3rd ed., John Wiley and Sons, New York.

U.S. Steel. (1969). “Steel sheet piling design manual,” Pittsburgh, PA.

Wang, S-T., and Reese, L. C. (1986). "Study of design methods for vertical drilled shaft retaining walls," Texas State Department of Highways and Pubic Transportation, Austin. 
Weatherby, D. E. (1982). “Tiebacks,” Report FHWA- RD-82-047, Federal Highway Administration, Washington, DC.

. (1998). "Design manual for permanent ground anchor walls," Report FHWA-RD-97-130, Federal Highway Administration, Washington, DC.

Weatherby, D. E., Chung, M., Kim, N-K., and Briaud, J-L. (1998). "Summary report of research on permanent ground anchor walls; II, Full-scale wall tests and a soil-structure interaction model," Report FHWA-RD-98-066, Federal Highway Administration, McLean, VA.

Winkler, E. (1867). "Die lehrevon elasizital and testigeit" ("On elasticity and fixity"), Prague, Czechoslovakia.

Wong, P. C., Kulhawy, F. H., and Ingraffea, A. R. (1989). "Numerical modeling of interface behavior for drilled shaft foundations under generalized loading." Foundation engineering: Current principles and practice, ASCE Geotechnical Special Publication 22, 565-79.

Xanthakos, Petros P. (1991). Ground anchors and anchored structures. John Wiley and Sons, New York.

Yoo, Chungsik. (2001). "Behavior of braced and anchored walls in soils overlying rock, Journal of Geotechnical and Geoenvironmental Engineering (ASCE), 127(3), 225-33.

Zaman, M. M., Desai, C. S., and Drumm, E. C. (1984). "Interface model for dynamic soil-structure interaction," Journal of Geotechnical Engineering, 110(9), 1257-73. 


\section{Appendix A Relevant Design Standards, Specifications, and Computer Programs}

\section{Government Publications}

American Association of State Highway and Transportation Officials, Washington, DC

\begin{tabular}{ll}
\hline $\begin{array}{l}\text { Reference } \\
\text { Designation }\end{array}$ & Title \\
\hline \hline AASHTO-1996 & Standard Specifications for Highway Bridges \\
AASHTO-AGC- & Joint Committee Task Force 27 Report, Specification for Permanent \\
ARTBA-1990 & Ground Anchors
\end{tabular}

\section{Department of the Army - Engineer Manuals}

\begin{tabular}{ll}
\hline $\begin{array}{l}\text { Reference } \\
\text { Designation }\end{array}$ & Title \\
\hline \hline EM 1110-1-1804 & Geotechnical Investigations \\
EM 1110-1-1905 & Bearing Capacity of Soils \\
EM 1110-1-1906 & Soil Sampling \\
EM 1110-1-2908 & Rock Foundations \\
EM 1110-2-1906 & Laboratory Soils Testing \\
EM 1110-2-2104 & Strength Design for Reinforced-Concrete Hydraulic Structures \\
EM 1110-2-2105 & Design of Hydraulic Steel Structures \\
EM 1110-2-2502 & Retaining and Flood walls \\
EM 1110-2-2504 & Design of Sheet Pile Walls \\
EM 1110-2-2906 & Design of Pile Foundations \\
\hline
\end{tabular}




\section{U.S. Department of Transportation \\ Federal Highway Administration \\ 6300 Georgetown Pike \\ McLean, VA 22101-2296}

\begin{tabular}{|c|c|}
\hline $\begin{array}{l}\text { Reference } \\
\text { Designation }\end{array}$ & Title \\
\hline FHWA-DP-68-1R & Permanent Ground Anchors \\
\hline FHWA-DP-90-068 & $\begin{array}{l}\text { DP-68 Permanent Ground Anchors; Vol 2, Field Demonstration Project } \\
\text { Summaries }\end{array}$ \\
\hline FHWA-DP-96-69R & Manual for Design and Construction Monitoring of Soil Nail Walls \\
\hline FHWA-HI-97-013 & Design and Construction of Pile Driven Foundations; Vol 1 \\
\hline FHWA-HI-99-007 & Earth Retaining Structures Participant Manual \\
\hline FHWA-RD-81-150 & Permanent Ground Anchors, Soletanche Design Criteria \\
\hline FHWA-RD-81-151 & Permanent Ground Anchors, Nicholson Design Criteria \\
\hline FHWA-RD-82-047 & Tiebacks \\
\hline FHWA-RD-97-130 & Design Manual for Permanent Ground Anchor Walls \\
\hline FHWA-RD-98-065 & $\begin{array}{l}\text { Summary Report of Research on Permanent Ground Anchor Walls; } \\
\text { Vol I: Current Practice and Limit Equilibrium Analysis }\end{array}$ \\
\hline FHWA-RD-98-066 & $\begin{array}{l}\text { Summary Report of Research on Permanent Ground Anchor Walls; } \\
\text { Vol II: Full Scale Wall Tests and a Soil Structure Interaction Model }\end{array}$ \\
\hline FHWA-RD-98-067 & $\begin{array}{l}\text { Summary Report of Research on Permanent Ground Anchor Walls; } \\
\text { Vol III: Model-Scale Wall Tests and Ground Anchor tests }\end{array}$ \\
\hline FHWA-RD-98-068 & $\begin{array}{l}\text { Summary Report of Research on Permanent Ground Anchor Walls; } \\
\text { Vol IV: Conclusions and Recommendations }\end{array}$ \\
\hline FHWA-RD-98-093 & $\begin{array}{l}\text { Tieback Wall - Anchored Wall Design and Analysis Program for Personal } \\
\text { Computers }\end{array}$ \\
\hline FHWA-SA-91-043 & The Cone Penetrometer Test \\
\hline FHWA-SA-91-044 & The Flat Dilatometer Test \\
\hline FHWA-SA-91-048 & $\begin{array}{l}\text { COM624P: Laterally Loaded Pile Analysis Program for the } \\
\text { Microcomputer }\end{array}$ \\
\hline FHWA-SA-96-072 & $\begin{array}{l}\text { Corrosion/Degradation of Soil Reinforcements for Mechanically } \\
\text { Stabilized Walls }\end{array}$ \\
\hline FHWA-SA-97-076 & Geotechnical Engineering Circular No. 3 \\
\hline FHWA-SA-99-015 & Geotechnical Engineering Circular No. 4 \\
\hline FHWA-SA-99-019 & Drilled Shafts: Construction Procedures and Design Methods \\
\hline FHWA-SA-96-038 & Geotechnical Engineering Circular No. 2 \\
\hline
\end{tabular}




\title{
Nongovernment Publications
}

\author{
American Concrete Institute (ACl) \\ PO Box 9094 \\ Farmington Hills, MI 48333-9094
}

\begin{tabular}{ll}
\hline Reference Designation & Title \\
\hline \hline $\mathrm{ACl}$ Committee 201 Report & Guide to Durable Concrete \\
$\mathrm{ACl}$ Committee 224 Report & Control of Cracking in Concrete Structures \\
$\mathrm{ACl}$ Committee 224.3 Report & Joints in Concrete Construction \\
$\mathrm{ACl}$ Committee 301 & Specifications for Structural Concrete \\
$\mathrm{ACl}$ Committee 315 Report & Details and Detailing of Concrete Reinforcement \\
$\mathrm{ACl} 318$ & Building Code Requirements for Structural Concrete and \\
$\mathrm{ACl}$ Committee 336 Report & Commentary \\
$\mathrm{ACl}$ Committee 350 Report & Envindard Specification for the Construction of Drilled Piers \\
$\mathrm{ACl}$ Committee 439 Report & Mechanical Connections of Reinforcing Bars \\
$\mathrm{ACl}$ Committee 449 Report & Concrete Nuclear Structures, Appendix B, Steel Embedments \\
$\mathrm{ACl}$ Committee 543 Report & Recommendations for the Design Manufacture, and \\
ACl Committee Report & Corrosion of Metals in Concrete
\end{tabular}

\section{American Forest and Paper Association (AFPA) 1111 19th Street NW, Suite 700 Washington, DC 20036}

\begin{tabular}{ll}
\hline Reference Designation & Title \\
\hline \hline NDS-91 & National Design Specification for Wood Construction \\
AF\&PA/ASCE 16 & Standard for Load and Resistance Factor Design (LRFD) for \\
& Engineered Wood Construction
\end{tabular}

\section{American Institutes of Steel Construction, Inc. (AISC) One East Wacker Drive, Suite 3100 Chicago, IL 60601-2001}

\begin{tabular}{ll}
\hline Reference Designation & Title \\
\hline \hline AISC Manual of Steel Construction & Load and Resistance Factor Design \\
AISC Manual of Steel Construction & Allowable Stress Design
\end{tabular}




\section{American Society for Testing and Materials (ASTM) 100 Barr Harbor Drive West Conshohocken, PA 19248-2959}

\begin{tabular}{ll}
\hline Reference Designation & Title \\
\hline \hline ASTM A36 & Specification for Carbon Structural Steel \\
ASTM A307 & Carbon Steel Bolts and Studs, 60,000 psi Tensile Strength \\
ASTM A325 & Structural Bolts, Steel, Heat treated, 120/105 Ksi Minimum Tensile \\
& Strength \\
ASTM A328 & Specifications for Steel Sheet Piling \\
ASTM A416 & Specifications for Uncoated Stress-Relieved Strand for Prestressed \\
& Concrete \\
ASTM A490 & Structural Joints Using ASTM A325 or A490 Bolts \\
ASTM A572 & High-Strength Low-Alloy Columbium-Vanadium Steels of Structural \\
& Quality \\
ASTM A690 & Specifications for High-Strength, Low-Alloy Steel H-Piles and Sheet \\
ASTM A706 & Piling for Use in Marine Environments \\
ASTM A709 & Low-Alloy Steel Deformed Bars for Concrete Reinforcement \\
ASTM A722 & Structural Steel for Bridges. \\
ASTM D512 & Specifications for Uncoated High-Strength Bar for Prestressed \\
ASTM D516 & Concrete \\
ASTM D653 & Standard Test Methods for Chloride lon In Water \\
ASTM D854 & Standard Test Method for Sulfate lon in Water \\
ASTM D1556 & Standard Terminology Relating to Soil, Rock, and Contained Fluids \\
& Standard Test Methods for Specific Gravity of Soil Solids by Water \\
ASTM D4254 & Pycnometer \\
ASTM G51 & Standard Test Method for Density and Unit Weight of Soil in Place by \\
the Sand-Cone Method & Standard Test Methods for Minimum Index Density and Unit Weight \\
ASTM G57 & of Soils and Calculation of Relative Density \\
Reston, VA & Testing \\
& Standard Test Method for Field Measurement of Soil Resistivity \\
Using the Wenner Four-Electrode Method
\end{tabular}

\begin{tabular}{ll}
\hline Reference Designation & Title \\
\hline \hline ANSI/ASCE 7-95 & Minimum Design Loads for Buildings and Other Structures \\
ASCE 2000 & SEI/ASCE Technical Committee on Performance of Structures \\
& During Construction Effective Analysis of Diaphragm Walls \\
Geotechnical Special & ASCE Specialty Conference on Design and Performance of Earth \\
Publication No. 25 & Retraining Structures \\
Geotechnical Special & Guidelines of Engineering Practice for Braced and Tied-Back \\
Publication No. 74 & Excavations \\
Geotechnical Special & Design and Construction of Earth Retaining Systems \\
Publication No. 83 &
\end{tabular}




\section{American Welding Society (AWS) 550 NW LeJeune Road \\ Miami, FL 33126}

\begin{tabular}{ll}
\hline Reference Designation & Title \\
\hline \hline AWS D1.1 & Structural Welding Code - Steel \\
AWS D1.4 & Structural Welding Code - Reinforcing Steel
\end{tabular}

National Association of Corrosion Engineers (NACE)

PO Box 218340

Houston TX 77218

\begin{tabular}{ll}
\hline Reference Designation & Title \\
\hline \hline NACE & Corrosion Handbook.
\end{tabular}

Prestressed Concrete Institute ( $\mathrm{PCl})$

175 W. Jackson Boulevard, Suite 1859

Chicago, IL 60604-9773

\begin{tabular}{ll}
\hline Reference Designation & Title \\
\hline \hline $\mathrm{PCl}$ & Design Handbook
\end{tabular}

Post-Tensioning Institute (PTI)

1717 W. Northern Avenue

Phoenix, AZ 85021

\begin{tabular}{ll}
\hline Reference Designation & Title \\
\hline \hline PTI & Post-Tensioning Manual, 5th edition \\
PTI & Recommendations for Prestressed Rock and Soil Anchors
\end{tabular}

\section{Computer Programs}

\section{Department of the Army - Computer Programs}

\begin{tabular}{ll}
\hline Reference Designation & Title \\
\hline \hline CBEAMC & Computer Program for the Analysis of Beam-Column Structures with \\
& Nonlinear Supports \\
CWALSSI & Computer Program for Winkler Soil-Structure Interaction Analysis of \\
SOILSTRUCT & Sheet-Pile Walls \\
& Computer Program for the Incremental Construction, Soil-Structure \\
& Interaction Analyses
\end{tabular}




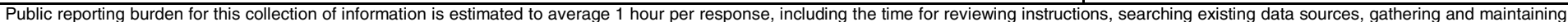

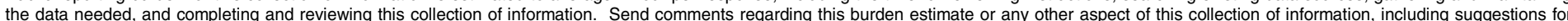

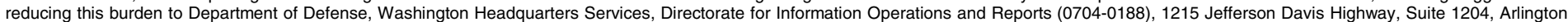

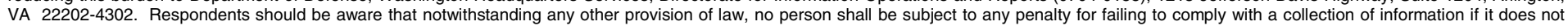
display a currently valid OMB control number. PLEASE DO NOT RETURN YOUR FORM TO THE ABOVE ADDRESS.
1. REPORT DATE (DD-MM-YYYY) 2. REPORT TYPE
3. DATES COVERED (From - To) Final report

December 2001

\section{TITLE AND SUBTITLE}

State of the Practice in the Design of Tall, Stiff, and Flexible Tieback Retaining Walls

5a. CONTRACT NUMBER

5b. GRANT NUMBER

5c. PROGRAM ELEMENT NUMBER

5d. PROJECT NUMBER

6. AUTHOR(S)

Ralph W. Strom, Robert M. Ebeling

5e. TASK NUMBER

5f. WORK UNIT NUMBER

INP 33272

\section{PERFORMING ORGANIZATION NAME(S) AND ADDRESS(ES)}

9474 SE Carnaby Way, Portland, OR 97266;

U.S. Army Engineer Research and Development Center, Information Technology

Laboratory, 3909 Halls Ferry Road, Vicksburg, MS 39180-6199

8. PERFORMING ORGANIZATION REPORT NUMBER

ERDC/ITL TR-01-1

9. SPONSORING / MONITORING AGENCY NAME(S) AND ADDRESS(ES)

U.S. Army Corps of Engineers, Washington, DC 20314-1000

10. SPONSOR/MONITOR'S ACRONYM(S)

11. SPONSOR/MONITOR'S REPORT NUMBER(S)

TR INP-01-4

\section{DISTRIBUTION / AVAILABILITY STATEMENT}

Approved for public release; distribution is unlimited

\section{SUPPLEMENTARY NOTES}

\section{ABSTRACT}

In tieback wall design, the determination of anchor loads and wall forces requires knowledge about the interaction between the wall and the soil during successive stages of excavation, as well as after completion of the project. Interaction between the wall and soil is difficult to predict. As a result, simple methods of analysis have been developed for use in the design of various tieback wall systems. These methods may, or may not, require a construction sequencing analysis.

This report describes state-of-the-practice analytical methods used to evaluate tieback wall performance and to design the tieback wall and ground anchor system. Analytical methods include equivalent beam on rigid support methods, beam on elastic foundation methods, and finite element methods.

The applicability of the various design methods with respect to various tieback wall systems frequently used on Corps projects is described in the report. Tieback wall systems covered in the report include vertical sheet-pile systems, soldier beam systems with wood or concrete lagging, secant cylinder pile systems, reinforced concrete slurry wall systems, and slurry wall systems constructed using soldier beams and concrete lagging.

Analysis methods depend on whether the tieback wall system is stiff or flexible. The report describes the characteristics of stiff and flexible tieback wall systems and indicates how the analysis method selected can be influenced by wall stiffness.

\section{SUBJECT TERMS}

Allowable stress design

Anchored wall

16. SECURITY CLASSIFICATION OF:

\section{a. REPORT}

UNCLASSIFIED b. ABSTRACT

UNCLASSIFIED
Ground anchor

Retaining wall

Soldier beam

Tieback wall

\begin{tabular}{|l|c|l|} 
17. LIMITATION & $\begin{array}{l}\text { 18. NUMBER } \\
\text { OF ABSTRACT }\end{array}$ & $\begin{array}{l}\text { 19a. NAME OF RESPONSIBLE } \\
\text { OFERSON }\end{array}$ \\
\cline { 3 - 3 } & 251 & $\begin{array}{l}\text { 19b. TELEPHONE NUMBER (include } \\
\text { area code) }\end{array}$ \\
& &
\end{tabular}

